\title{
Dynamics, equilibria, and values
}

Citation for published version (APA):

Joosten, R. A. M. G. (1996). Dynamics, equilibria, and values. [Doctoral Thesis, Maastricht University]. Universiteit Maastricht. https://doi.org/10.26481/dis.19961212rj

Document status and date:

Published: 01/01/1996

DOI:

10.26481/dis.19961212rj

Document Version:

Publisher's PDF, also known as Version of record

\section{Please check the document version of this publication:}

- A submitted manuscript is the version of the article upon submission and before peer-review. There can be important differences between the submitted version and the official published version of record.

People interested in the research are advised to contact the author for the final version of the publication, or visit the DOI to the publisher's website.

- The final author version and the galley proof are versions of the publication after peer review.

- The final published version features the final layout of the paper including the volume, issue and page numbers.

Link to publication

\footnotetext{
General rights rights.

- You may freely distribute the URL identifying the publication in the public portal. please follow below link for the End User Agreement:

www.umlib.nl/taverne-license

Take down policy

If you believe that this document breaches copyright please contact us at:

repository@maastrichtuniversity.nl

providing details and we will investigate your claim.
}

Copyright and moral rights for the publications made accessible in the public portal are retained by the authors and/or other copyright owners and it is a condition of accessing publications that users recognise and abide by the legal requirements associated with these

- Users may download and print one copy of any publication from the public portal for the purpose of private study or research.

- You may not further distribute the material or use it for any profit-making activity or commercial gain

If the publication is distributed under the terms of Article $25 \mathrm{fa}$ of the Dutch Copyright Act, indicated by the "Taverne" license above, 


\section{Dynamics, Equilibria, and Values}

\section{PROEFSCHRIFT}

ter verkrijging van de graad van doctor aan de Universiteit Maastricht, op gezag van de Rector Magnificus, Prof. mr. M.J. Cohen, volgens het besluit van het College van Dekanen, in het openbaar te verdedigen op donderdag 12 december 1996 om 16.00 uur

door

Reinoud Anna Maria Gerardus Joosten 


\title{
Promotores:
}

Prof.dr. H.J.M. Peters

Prof.dr.ir.drs. O.J.Vrieze

\section{Co-promotor:}

\author{
Dr. F. Thuijsman
}

Beoordelingscommissie:

\author{
Prof.dr. F.C. Palm (voorzitter) \\ Dr. J.J.M. Derks \\ Dr. M.J.M. Jansen \\ Prof.dr. A.J.J. Talman (Katholieke Universiteit Brabant) \\ Prof.dr. S.H. Tijs
}


אתה רונן לאדם דעת ומלמד לאנוש ביצגה.

וחנט מאתך

רצה בינה ו"השכלי.

ברוך אתה "י חונך הדצת.

Gij begenadigt de mens met weten

en leert de sterveling inzicht.

Begenadig ons van uwentwege

met weten, inzicht en verstand.

Gezegend gij, Heer, die begenadigt met weten. 


\section{Acknowledgements}

I thank my supervisors Hans Peters and Frank Thuijsman. They taught me the trade, or at least a trade. Now, after five, almost six, years of their influencing me, I find myself looking upon the writings of myself and others with their eyes. I am amazed of how much of their advice, remarks and criticism I seem to have internalized. They performed a remarkable high-wire act by giving me freedom and encouragement, beneficial to my mental health, simultaneously giving me criticism and guidance, beneficial to my work.

Dolf Talman gave my phone number to Hans which led to my being hired. There is more: the strain of research which we began in Tilburg while I was finishing my Master's degree under Dolf's supervision, continues until today. To our mutual satisfaction, I believe. I thank Koos Vrieze for joining Frank and Hans in supervising me. Luc Soete sought my cooperation for an article, which drew more reactions from colleagues and friends than the entire rest of my research. Eric van Damme is thanked for giving me his book on Nash equilibria, and for his encouragement. Jean-Jacques Herings commented on a paper of Dolf and mine. It benefitted much from his remarks. Antoon van den Elzen's advice and encouragement is remembered. The reading committee is thanked for pointing out a few tricky details to me.

My roommates at MERIT, Theon van Dijk and Maureen Lankhuizen, have contributed more than they can imagine. I shared more with them than just my room. These matches were not made in heaven, but by a mortal called Wilma Coenegrachts, who should consider a career in the match-making business. Wilma has helped me in many matters, but I should mention her assistance in organizing the MERIT-seminars. My colleagues at MERIT and at the Department of Mathematics created a fine academic and personal climate. Crucial for a research institute is the quality and spirit of its supporting cast. I only mention the present members of MERIT's traditionally strong team: Silvana de Sanctis, Ellen Nelissen and Corien Gijsbers.

I thank NWO for sponsoring a trip of mine to the Summer School in Economic Theory in Jerusalem, I thank the organizers of the Jerusalem summer school for a grant on another trip. METEOR is my new employer, I am grateful for the opportunity to work out topics contained in this thesis. My shadow career, some might say: shady, is in basketball, I thank LV\&S of Tilburg University, as well as all the other people I cooperated with in this sphere of my professional and personal life.

I thank my parents and my 'little' brother for support. Dorine shared my life the last seven years, as I shared hers with her. I think I have been the main beneficiary of the relationship, though it has never been a null-sum game as very strong positive externalities and complementarities exist. She beat me in producing her own 'booklet(s)', a process which I inadvertently(?) sabotaged by deleting her files. 


\section{Contents}

1 A readler's guide to the thesis $\quad 7$

2 Economic adjustment processes 11

2.1 Introduction . . . . . . . . . . . . . . 11

2.2 Model and preliminaries . . . . . . . . . . . . . . 16

2.3 Paths of price adjustment processes . . . . . . . . . 25

2.4 Globally convergent price adjustment ... . . . . . . . 30

2.5 A globally convergent algorithm ... . . . . . . . . 35

2.5.1 Description of the algorithm . . . . . . . . 35

2.5.2 The steps of the algorithm . . . . . . . . . . . . 39

2.6 An intersection theorem ................ . . . 44

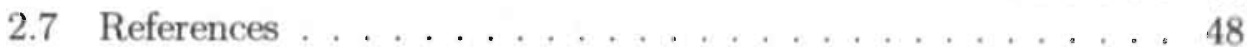

3 Evolution of populations and strategies 53

3.1 Introduction . . . . . . . . . . . . . . . 53

3.2 The 'population dynamics' case . . . . . . . . . . . 58

3.2.1 The model and equilibrium concepts . . . . . . . 58

$3: 2.2$ Evolutionary dynamics and tâtonnements ...... 63

3.3 Population dynamics and myopic learning . . . . . . . 66 66

3.3 .1 The model . . . . . . . . . . . . . . . 66

3.3.2 Fixed points of the dynamical system . . . . . . . 71

3.3.3 Strategic stability versus dynamic stability . . . . . 76

3.4 Discussion ........................... 79

3.5 Appendix . . . . . . . . . . . . . . . . . 81

3.6 References .......................... 85

4 Changing payoffs or action sets 93

4.1 Introduction . . . . . . . . . . . . . . 93 
4.2 Zero-sum games with vanishing actions . . . . . . . . . 95 95

4.2.1 $\left(\mathrm{r}_{1}, \mathrm{r}_{2}\right)$-Restricted zero-sum games with $r_{1} \leq 2$ or $r_{2} \leq 2 \quad 96$

$4.2 .2 \quad(3,3)$-Restricted zero-sum games . . . . . . . . . . 100

4.2.3 On general $\left(r_{1}, r_{2}\right)$-restricted zero-sum games . . . . . . 104

4.3 General-sum games with vanishing actions . . . . . . . . . 107

4.3.1 A 'Folk Theorem' for $\left(r_{1}, r_{2}\right)$-restricted games . . . . 110

4.3.2 Existence of a Nash equilibrium for $\left(r_{1}, r_{2}\right)$ with $\mathrm{r}_{1} \leq 2$ or $\mathrm{r}_{2} \leq 2 \ldots \ldots \ldots \ldots \ldots \ldots \ldots \ldots \ldots$

4.4 Differential games with changing payoffs . . . . . . . . . 126

4.4.1 An investment problem . . . . . . . . . . . 126

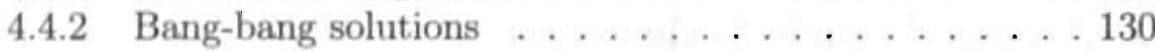

4.4 .3 A Cobb-Douglas case . . . . . . . . . . . . . . 131

4.4.4 An alternative formulation . . . . . . . . . . 133

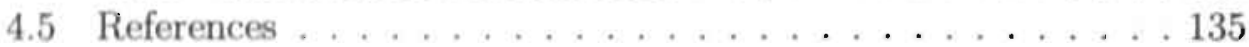

5 Egalitarianism, potentials, and values $\quad 139$

5.1 Introduction . . . . . . . . . . . . . . . . 139

5.2 The model and preliminaries . . . . . . . . . . . . . 141

$5.3 \alpha$-Egalitarian Shapley values . . . . . . . . . . . . . 147

5.4 Potentials, values, and axioms . . . . . . . . . . . 155

5.5 Appendix . . . . . . . . . . . . . . . . . . 160

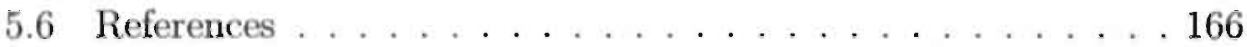

6 Addenda $r$

6.1 Samenvatting (Summary in Dutch) . . . . . . . . . . . 169

6.2 Curriculum vitae . . . . . . . . . . . . . . . . . 172

6.3 Author index . . . . . . . . . . . . . . . . . . 173 


\section{Chapter 1}

\section{A reader's guide to the thesis}

This thesis is a compilation of results on dynamics, evolution and (un)learning in economics and noncooperative game theory, as well as on a topic in cooperative game theory. The body of this thesis consists of four chapters which can be read independently. Here, we briefly describe the frameworks of the four chapters, our contributions, and possible connections between the chapters. This reader's guide aims to give a quick overview, for detailed descriptions of the separate chapters, we refer to the respective introductions.

Chapter 2 is called 'Economic adjustment processes' and treats a model in mathematical economics. At an economic equilibrium, the demand equals the supply of each commodity. The following three questions are traditionally regarded as important. Does there exist an equilibrium price vector? Can an equilibrium price vector be reached by adjusting prices of commodities starting from a situation where the demand does not equal the supply for each commodity, and if so, how? Can an equilibrium price vector be computed or approximated, and if so, how? These questions motivate our contributions. Firstly, we give a new price adjustment process, which may be used to reach an equilibrium price vector from any given initial price vector in an arbitrary exchange economy. Secondly, we contribute a new variable dimension restart algorithm to compute an equilibrium price vector in an arbitrary exchange economy. Thirdly, we prove existence of an economic equilibrium in an exchange economy by deriving an 'intersection theorem'.

Chapter 3 is called 'Evolution of populations and strategies', and it contains two models. The first model describes the evolution of the composition of a population with several subgroups, assuming that a process of 'Darwinian' selection takes place. At any point in time, changes in the compo- 
sition of the population are driven by differences in fitness levels among the subgroups given the strategic environment, which in turn changes in. general when the composition of the population changes. The second model in Chapter 3 describes the evolution of a population consisting of several subgroups as well, but it describes additionally how the subgroups adapt their strategies in order to improve their payoffs in the strategic environment. We interpret the strategical adjustment processes of the subgroups as learning dynamics. Again, the strategic environment changes in general as the composition of the population changes and the subgroups alter their strategies.

We use the concept of saturated equilibrium, and the new concepts of evolutionarily stable equilibrium and generalized evolutionarily stable state. We show that these evolutionary equilibria are rest points for the classes of evolutionary dynamics treated. Relations of these three evolutionary equilibrium concepts to standard fixed point concepts known from the analysis of dynamical systems, as well as to standard game theoretical equilibrium concepts are shown.

Connections between Chapters 2 and 3 are surprising. Evolutionary dynamics may be interpreted as a price adjustment process in a pure exchange economy with normalized prices. In line with this analogy, several fixed point concepts for evolutionary dynamics correspond with fixed point concepts for price adjustment processes. For example the aforementioned saturated equilibrium corresponds with the Walras equilibrium. Several algorithms to compute a Walras equilibrium in pure exchange economies, can also be used to compute a saturated equilibrium for a deterministic evolutionary model.

Chapter 4 is called 'Changing payoffs or action sets'. It contains two models where the payoffs to the players, or their action sets, may change during the play of the game. In the first model of Chapter 4, we study twoplayer repeated games with vanishing actions. In such a game, the number of actions which each player possesses, may decrease as play develops. If namely, an action has not been used by a player for a certain number of consecutive stages of the play, then this action is removed from this player's action set. This phenomenon of losing an action is referred to as 'unlearning by not doing'. We show existence of solutions to several classes of zero-sum, as well as general-sum games with vanishing actions, by giving explicit Nash equilibria and optimal strategies for the limiting average reward criterion.

In the second model of Chapter 4, we investigate aspects of changing payoffs by means of differential games. Two players receive streams of payoffs, a fixed amount of which is to be invested in two activities by each player. Each 
player has one activity of which the payoff only depends on his investment into that activity. The other activity depends not only on his investment into this activity, but also on the investment of the other player in this activity. Assuming that both players wish to maximize their infinite stream of payoffs, Nash equilibria and optimal open-loop strategies are derived.

Chapter 5 is called 'Egalitarianism, potentials, and values', and it deals with cooperative game theory. One of the questions which we try to answer is: How should possible gains, or costs, made by a group of cooperating players, be allocated? A 'value' for a transferable utility game can be regarded as a division-rule of the gains or costs made by a group of cooperating agents. A well-known solution in game-theory is the Shapley value, which has been applied not only to economics, but also to politics in order to quantify the power of agents in committees or parliament.

Our contribution is motivated by a wish to devise a value incorporating a notion of egalitarianism. We do this by assuming that null-players, i.e., players who do not contribute anything in the game, receive a fraction $\alpha$ of the per-capita 'income' in the situation where all players cooperate. This may come about by voluntary contributions to some fund, or by some form of taxation of the total income. Thus, the parameter $\alpha$ reflects the prevailing level of egalitarianism or solidarity. We consider values that satisfy this condition, called $\alpha$-egalitarianism, and several other 'reasonable' properties. This leads to axiomatic characterizations of a class of values, the ' $\alpha$-egalitarian Shapley values'. We proceed by giving several other axiomatic characterizations of each $\alpha$-egalitarian Shapley value.

We take the idea of 'taxing and redistributing' further to derive more general classes of values depending on a tuple of parameters. For this purpose, we make use of so-called 'potentials'. A potential in this context is a map which attributes to every game a unique real number. Given a tuple of parameters, we define a potential and connect to this potential a unique value as follows. Each player in the game receives under the value his marginal contribution (in some sense) to the potential of the game. We examine relations between restrictions on the tuple of parameters, and several properties of the value which is determined by this tuple. 



\section{Chapter 2}

\section{Economic adjustment processes}

\subsection{Introduction}

At a Walrasian equilibrium, the demand for each commodity is equal to the supply of that commodity. The question if and how an economic equilibrium can be reached starting from a situation where the economy is not in equilibrium, has attracted much attention. Given the crucial role of the prices of the commodities in the economy, it is hardly surprising that many contributions in the literature have focussed on price adjustment processes.

In the framework of an exchange economy Walras [1874] proposed a process where successively the price of one commodity is changed such that the market for that commodity reaches an equilibrium. It is not difficult to construct an exchange economy for which Walras' so-called successive tâtonnement process does not converge to an equilibrium price vector, implying that the process continues forever without reaching an equilibrium price vector (e.g., Scarf [1960]).

Samuelson [1947] formulated an improvement of Walras' successive tâtonnement process, the so-called simultaneous tâtonnement process, as a system of differential equations which formalize that the price of each commodity changes proportional to its excess demand at any point in time. As the name indicates, all prices are changed simultaneously throughout this process, hence information available from the markets of all commodities is used. For some time, scientific interest focussed on finding conditions to ensure the convergence of Samuelson's simultaneous tâtonnement process and related price adjustment processes, see e.g., Arrow \& Hurwicz [1958], Arrow et al. 
[1959], Uzawa [1961].

Scarf [1960] demonstrated that any project of finding realistic necessary and sufficient conditions for convergence of such price adjustment processes was bound to fail. Furthermore, the contributions of Sonnenschein [1972, 1973], Mantel [1974], and Debreu [1974] showed definitely and explicitly that any function satisfying continuity, Walras' law, and homogeneity of degree zero in prices, can be an excess demand function for an exchange economy on an arbitrarily large subset of the interior of the price space. Hence, any set of conditions on the excess demand functions beyond these three conditions, will not hold in general.

An alternative price adjustment process is the Global Newton method, proposed by Smale [1976]. This process is known to converge to an equilibrium price vector for arbitrary excess demand functions. During the process, the prices of the commodities are changed. simultaneously in such a manner that the absolute value of the excess demand of each commodity decreases. The Global Newton method must be started on the boundary of the price space to ensure convergence to an equilibrium price vector, which makes the economic interpretation of the path of prices generated by this process somewhat cumbersome.

Scarf $[1967,1973]$ and Kuhn $[1968,1969]$ formulated two types of processes for computing an equilibrium price vector. The sequence of price vectors gen-erated by each of these computational processes, forms a path of points in the unit simplex, which may be interpreted as a price adjustment process. Scarf's process is the dual of Kuhn's process in the following sense. For the former process, initially the price of the commodity which has the lowest excess demand is decreased, and a path of points in the unit simplex is generated satisfying the property that the excess demands for all other commodities, which have the highest excess demands, are kept approximately equal to each other. For Kuhn's process, however, the price of the commodity which has the highest excess demand is increased, and the prices of all other commodities, which have the lowest excess demands, are changed such that their excess demands are kept approximately equal to each other. Both. processes terminate at an equilibrium price vector for arbitrary exchange economies provided all goods are desirable. To ensure convergence, these processes must be started at a price vector, where one or more commodities have prices equal to zero. An economic interpretation of these computational processes as price adjustment processes is therefore not straightforward.

Several contributions have appeared in the literature each linking a glob- 
ally convergent price adjustment process to a globally convergent computational process (e.g., Van der Laan \& Talman [1987a], Kamiya [1990]). A process is called globally convergent if it converges to an equilibrium price vector for arbitrary excess demand functions while being started at an arbitrary price vector. One class of globally convergent computational processes is the class of variable dimension restart algorithms. This class originated with the contribution of Van der Laan \& Talman [1979]. The first round of this algorithm may start with a rather large step size, and this round terminates quickly with a first approximation of an equilibrium price vector. If the excess demand at this approximation does not fulfil a given accuracy criterion, the procedure is restarted near the approximation with a smaller step size. The second round terminates with another approximation of an equilibrium price vector, and in general the excess demand at this price vector is closer to zero in each component than the approximation found in the first. round. Each round terminates in a finite number of steps with an approximation of an equilibrium price vector. Whenever the excess demand function at this approximation does not fulfil the given accuracy criterion, the procedure is restarted with a smaller step size. In this manner, an approximation of an equilibrium price vector can be found such that the excess demand function evaluated at this price vector, fulfils the arbitrary predetermined accuracy criterion.

Several improvements and generalizations of the original variable dimension restart algorithm of Van der Laan \& Talman [1979] have been developed, e.g., Doup \& Talman [1987a], Van der Laan et al. [1987] and Doup et al. [1987]. By these contributions, considerable gains in computational efficiency have been accomplished. Furthermore, several of these algorithms generate paths of prices which allow for quite natural interpretations as paths of a price adjustment process. Recent contributions exploit this observation, e.g., Van der Laan \& Talman [1987a,b], Van den Elzen [1993], Herings [1994,1995]. The aforementioned globally convergent price adjustment process of Kamiya [1990] does not belong to the class of price adjustment processes related to a variable dimension restart algorithm. In Kamiya's process namely, a homotopy function is introduced which deforms a trivial system having a unique solution, to the system of the excess demand function.

In this chapter we present a new variable dimension restart algorithm, as well as a new price adjustment process. Both processes are globally convergent. The price adjustment process may be regarded as a synthesis between the processes of Scarf and Kuhn. Contrary to these processes however, it may 
be started in an arbitrary price vector. In general, the starting point of the price adjustment process will not be an equilibrium price vector. Initially, the price of the commodity having the highest excess demand is increased in our price adjustment process, similar to Scarf's process. Simultaneously, the price of the commodity having the highest excess supply, i.e., the lowest excess demand, is decreased, similar to Kuhn's process. In general, under the price adjustment process the prices of the commodities with highest excess demand are allowed to increase from their initial level, and the prices of the commodities with lowest excess demand are allowed to decrease from their initial level. The prices of all commodities with highest and lowest excess demands are changed in such a manner that the excess demands of the commodities with highest excess demand are kept equal to each other, and the excess demands of the commodities with lowest excess demand are also kept equal to each other. The prices of the commodities neither having highest excess demand nor lowest excess demand, are kept equal to their initial levels, while their excess demands are allowed to vary between the minimal and the maximal levels. As soon as the excess demand of a certain commodity becomes maximal or minimal, its price is increased or decreased respectively, and its excess demand is kept maximal or minimal. Whenever the price of a commodity which has been raised or lowered from its initial level becomes equal to this initial level again, its price is kept equal to this initial level and its excess demand is allowed to become less than maximal or more than minimal, respectively.

In the case that there are $n+1$ commodities in an exchange economy, the variable dimension restart algorithm which we present in this chapter, leaves the starting point along one of $n(n+1)$ possible one-dimensional sets or rays. The algorithm is therefore referred to as the $n(n+1)$-ray algorithm, as it has become customary to distinguish the variable dimension restart algorithms by the number of rays along which the starting point may be left. In this class there exist: a 2-ray algorithm (Doup \& Talman [1987b]), several $(n+1)$ ray algorithms (e.g., Van der Laan \& Talman [1979,1980], Doup \& Talman [1987a]), and a (2 $\left.2^{n+1}-2\right)$-ray algorithm (Doup et al. [1987]). The $n(n+1)$ ray algorithm uses a simplicial subdivision of the unit simplex and generates a. piecewise linear path of points in a sequence of adjacent simplices, which leads from the starting point to an approximation of an equilibrium price vector. When taking identical starting price vectors, the path of prices generated by the $n(n+1)$-ray algorithm approximates the path of prices followed by the price adjustment process. How closely the path of the $n(n+1)$-ray algorithm. 
approximates the path followed by the price adjustment process, depends crucially on the subdivision of the unit simplex used by the algorithm.

We furthermore present a new intersection theorem in this chapter. Intersection theorems are used to prove the existence of solutions to mathematical programming problems, economic equilibrium existence problems, and existence of solutions to game-theoretical problems. A well-known intersection theorem is the KKM-lemma by Knaster, Kuratowski \& Mazurkiewicz [1929]. Another well-known intersection theorem is due to Freidenfelds [1974], and the latter result can be interpreted as a dual to the KKM-lemma. For surveys on intersection theorems on the unit simplex and on the simplotope, we refer to Talman [1994] and Van der Laan \& Talman [1993]. Recently, Herings [1995] and Yang [1996] have added new intersection results. A standard tool in proving intersection theorems is Kakutani's fixed point theorem. However, simplicial variable dimension algorithms may also be used to provide constructive proofs of existence of intersection points of a certain collection of closed subsets covering the unit simplex or the simplotope. We use the $n(n+1)$-ray algorithm to prove existence of an intersection point of a collection of closed subsets covering the unit simplex. Our intersection theorem generalizes the KKM-lemma and the intersection result of Freidenfelds on the unit simplex in the sense that these results form corollaries to it. Furthermore, the intersection theorem immediately leads to an alternative proof of existence of an economic equilibrium.

This chapter is organized as follows. In the following section, we formulate the underlying model of an exchange economy and several other concepts relevant for a description of our price adjustment process and our $n(n+1)$-ray variable dimension restart algorithm. In Section 2.3, we discuss computational processes, price adjustment processes, and convergence properties of these processes rather informally. We describe the paths followed by these processes and compare them to the paths which will be followed by our price adjustment process. As already stated our $n(n+1)$-ray algorithm approximately follows the path of our price adjustment process. In Section 2.4, we formulate the globally convergent price adjustment process. We demonstrate how the price adjustment process works by means of a detailed description of an example. In Section 2.5, we describe and formulate the $n(n+1)$-ray variable dimension restart algorithm. We demonstrate how the algorithm works by a detailed example. In Section 2.6, we prove the new intersection theorem. We show that the KKM-lemma and an intersection result of Freidenfelds follow immediately from our intersection theorem. We furthermore 
give an alternative proof of existence of an economic equilibrium based on our intersection theorem.

This chapter is based on Joosten \& Talman [1993] and on Joosten \& Talman [1995].

\subsection{Model and preliminaries}

We consider an exchange economy with $n+1$ commodities, indexed $j \in$ $I^{n+1}=\{1, \ldots, n+1\}$. The set of real numbers is denoted by $\Re$, the innerproduct of for instance $x, y \in \Re^{n+1}$ is denoted by $x \cdot y$. The set of all possible price vectors is $\Re_{+}^{n+1} \backslash\left\{0^{n+1}\right\}$, where $0^{n+1}$ is the $(n+1)$-vector consisting of zeroes. Let $\|x\|_{2}=\sqrt{\sum_{j=1}^{n+1} x_{i}^{2}}$ denote the Euclidean norm, and let $d_{2}(x, y)=$ $\|x-y\|_{2}$ denote the Euclidean distance for $x, y \in \Re^{n+1}$. Suppose that the economy has $m$ agents (or consumers). Each agent is characterized by his consumption set, initial endowments and preference relation. For each agent $i \in I^{m}=\{1, \ldots, m\}$ we assume that the following holds:

a. The consumption set $X^{i}$ is a compact, convex subset of $R_{+}^{n+1}$, containing the set.

$$
\left\{x \in \Re^{n+1} \mid 0 \leq x_{j} \leq \sum_{h=1}^{m} w_{j}^{h}+1 \text {, for all } j \in I^{n+1}\right\},
$$

where $w^{h}=\left(w_{1}^{h}, \ldots, w_{n+1}^{h}\right)$ is the vector of initial endowments of the $n+1$ commodities for consumer $h \in I^{m}$;

b. $w_{j}^{i}>0$ for all $j \in I^{n+1}$;

`. The preference relation $\succeq_{i}$ is continuous, monotonic and strictly convex.

Let $B^{i}(p)=\left\{x \in X^{i} \mid p \cdot x \leq p \cdot w^{i}\right\}$ denote the budget set of agent $i \in I^{m}$ given price vector $p \in \mathfrak{R}_{+}^{n+1} \backslash\left\{0^{n+1}\right\}$. We assume that each consumer $i \in I^{m}$ maximizes his utility over his budget set, i.e., he chooses a maximal element with respect to his preferences $\succeq_{i}$ in the budget set $B^{i}(p)$. Let

$$
x^{i}(p) \in\left\{\tilde{y} \in B^{i}(p) \mid \tilde{y} \succeq_{i} y \text { for all } y \in B^{i}(p)\right\} .
$$

be a vector of commodities which maximizes consumer $i$ 's utility given the price vector $p \in \Re_{+}^{n+1} \backslash\left\{0^{n+1}\right\}$. Under Assumptions (a)-(c), for every $p \in$ 
$\Re_{+}^{n+1} \backslash\left\{0^{n+1}\right\}$ this vector $x^{i}(p)$ is unique for every $i \in I^{m}$, moreover it is a boundary element of the budget set. Therefore,

$$
p \cdot x^{i}(p)=p \cdot w^{i} \text {, for each consumer } i \in I^{m} \text {, for every } p \in \Re_{+}^{n+1} \backslash\left\{0^{n+1}\right\} .
$$

Furthermore, the demand function $x^{i}: \Re_{+}^{n+1} \backslash\left\{0^{n+1}\right\} \rightarrow \Re^{n+1}$ defined pointwise by the above, is continuous in $p \in \Re_{+}^{n+1} \backslash\left\{0^{n+1}\right\}$ for each consumer $i \in I^{m}$.

Let $z^{i}: \Re_{+}^{n+1} \backslash\left\{0^{n+1}\right\} \rightarrow \Re^{n+1}$, be the excess demand function of consumer $i \in I^{m}$, defined by

$$
z^{i}(p)=x^{i}(p)-w^{i} \text { for every } p \in \Re_{+}^{n+1} \backslash\left\{0^{n+1}\right\} .
$$

Then, $p \cdot z^{i}(p)=0$ for every $p \in \Re_{+}^{n+1} \backslash\left\{0^{n+1}\right\}$. The excess demand function, defined by

$$
z(p)=\sum_{i \in I^{m}} z^{i}(p) \text { for every } p \in \Re_{+}^{n+1} \backslash\left\{0^{n+1}\right\},
$$

is a continuous function satisfying the following properties:

i. $p \cdot z(p)=0$ for all $p \in \Re_{+}^{n+1} \backslash\left\{0^{n+1}\right\}$ (Walras' law),

ii. $z_{j}(p) \geq 0$ whenever $p_{j}=0$ (desirability),

iii. $z(\mu p)=z(p)$ for all scalars $\mu>0$ and all $p \in \Re_{+}^{n+1} \backslash\left\{0^{n+1}\right\}$ (homogeneity of degree zero in prices).

The price vector $p^{*} \in \Re_{+}^{n+1} \backslash\left\{0^{n+1}\right\}$ is called an equilibrium price vector if $z\left(p^{*}\right)=0^{n+1}$. Sonnenschein [1972, 1973], Mantel [1974], and Debreu [1974] showed that Properties (i)-(iii) characterize excess demand functions. Property (iii) allows normalization of the price space to the $n$-dimensional unit simplex. The $\mathbf{n}$-dimensional unit simplex is given by:

$$
S^{n}=\left\{p \in \Re^{n+1} \mid p_{j} \geq 0 \text { for all } j \in I^{n+1} \text {, and } \sum_{j=1}^{n+1} p_{j}=1\right\} \text {. }
$$

The following states the well-known fact that there always exists an equilibrium price vector on the $n$-dimensional unit simplex for any excess demand function. Its proof is straightforward and is left to the reader. 


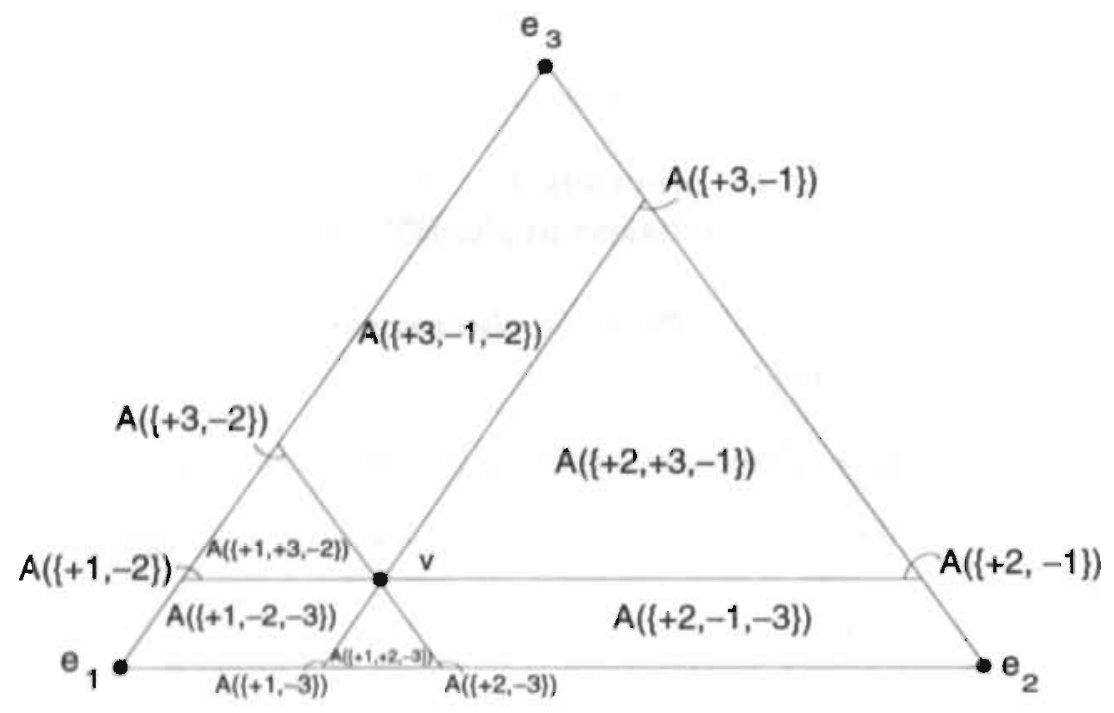

Figure 2.1: Illustration of Definition 2.2: The subdivision of $S^{2}$ into sets $A(T)$ of varying dimension.

Proposition 2.1 Let $z: S^{n} \rightarrow \Re^{n+1}$ be an excess demand function. Then, there exists $p^{*} \in S^{n}$ satisfying $z\left(p^{*}\right)=0^{n+1}$.

To facilitate the exposure in the following sections, we introduce several notations here. For $A \subset I^{n+1},-A$ is defined to be the set $\{-j \mid j \in A\}$. Furthermore, let $T$ be a subset of $I^{n+1} \cup-I^{n+1}$, satisfying $T \cap I^{n+1} \neq \emptyset$, $T \cap-I^{n+1} \neq \emptyset$, and $T \cap-T=\emptyset$. Let $T^{+}=\left\{j \in I^{n+1} \mid j \in T\right\}, T^{-}=\{j \in$ $\left.I^{n+1} \mid-j \in T\right\}$, and $T^{c}=\left\{h \in I^{n+1} \mid h \notin T \cup-T\right\}$.

Definition 2.2 Let $v \in$ int $S^{n}$ be given. For $T$ as described above, the set $\mathbf{A}(\mathbf{T})$ is given by:

$$
A(T)=\left\{p \in S^{n} \mid p_{j} \leq v_{j} \text { if } j \in T^{-}, p_{j}=v_{j} \text { if } j \in T^{c}, p_{j} \geq v_{j} \text { if } j \in T^{+}\right\} .
$$

The dimension of $A(T)$ is equal to $|T|-1$. Hence, $1 \leq \operatorname{dim} A(T) \leq n$ for all $T$ as described above. Furthermore, the union of the sets $A(T)$ over all $T \subset I^{n+1} \cup-I^{n+1}$ with $T^{c}=\emptyset$, is the unit simplex. The interpretation 


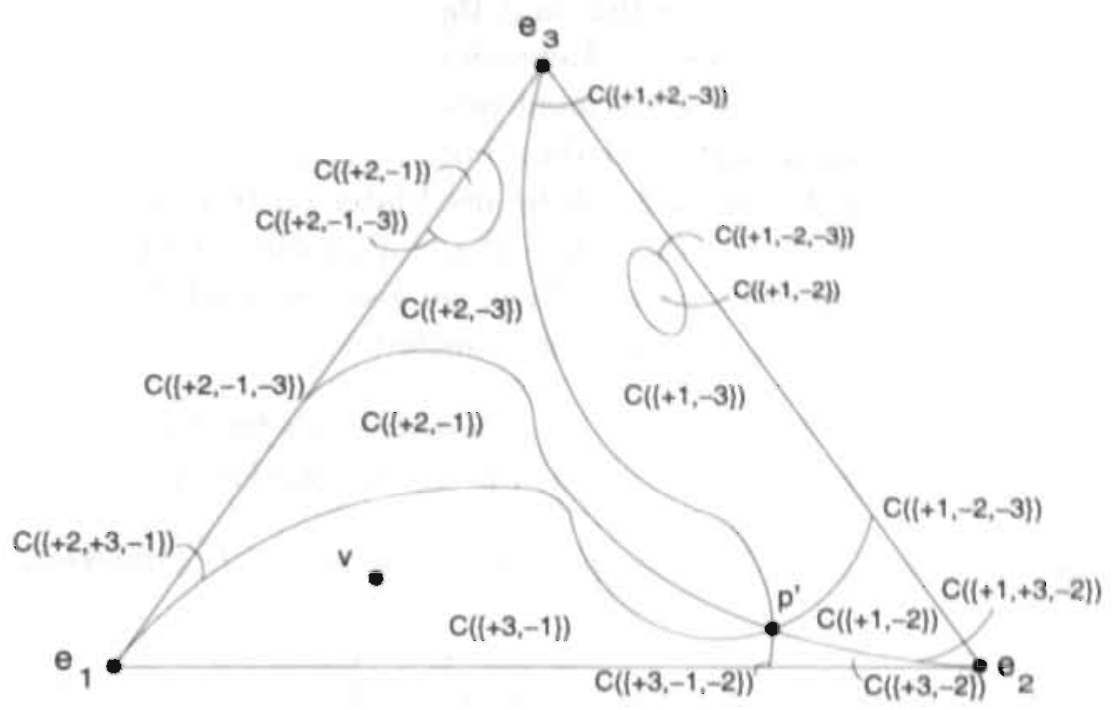

Figure 2.2: Illustration of Definition 2.3: the subdivision of $S^{2}$ into sets $C(T)$ of varing dimension.

of such a set $A(T)$ is straightforward. Any point in the interior of the set $A(T)$ may be reached from $v$ by increasing the prices of the commodities (of which the index corresponds with an index) in $T^{+}$, decreasing the prices of the commodities in $T^{-}$, while keeping the prices of the commodities in $T^{c}$ at their initial levels. For an illustration see Figure 2.1.

For an excess demand function $z: S^{n} \rightarrow \Re^{n+1}$, we define the following subsets of the $n$-dimensional unit simplex.

Definition 2.3 Let $z: S^{n} \rightarrow \Re^{n+1}$ be an excess demand function. Then, for $T$ as described above, the set $\mathbf{C}(\mathbf{T})$ is given by $C(T)=\left\{p \in S^{n} \mid\right.$

$$
\begin{array}{ll}
z_{j}(p)=\min _{h \in I^{n+1}} z_{h}(p) & \text { if } j \in T^{-}, \\
\min _{h \in I^{n+1}} z_{h}(p) \leq z_{j}(p) \leq \max _{h \in I^{n+1}} z_{h}(p) & \text { if } j \in T^{c}, \\
\left.z_{j}(p)\right)=\max _{h \in I^{n+1}} z_{h}(p) & \text { if } \left.j \in T^{+}\right\} .
\end{array}
$$

For an illustration, we refer to Figure 2.2. For the sake of comparison we have depicted the same point $v$ as in Figure 2.1. 
Since $v \in C(\{+3,-1\})$, it follows at the price vector $v$ that the excess demand of commodity 3 is maximal and the excess demand of commodity 1 is minimal. The sets defined by Definitions 2.2 and 2.3 , will be used for the globally convergent price adjustment process, as well as for the $n(n+1)$ ray variable dimension restart algorithm, both to be presented in the sequel. Furthermore, Figures 2.1 and 2.2 will be used later on to illustrate how our price adjustment process and the $n(n+1)$-ray algorithm reach an (approximation of) equilibrium price vector. Now, we give two useful lemmas, their proofs are straightforward and therefore omitted.

Lemma 2.4 Let $z: S^{n} \rightarrow \Re^{n+1}$ be an excess demand function. Let $p^{*} \in S^{n}$ satisfy $z_{j}\left(p^{*}\right)=\beta$ for all $j \in I^{n+1}$. Then $p^{*}$ is an equilibrium price vector.

Lemma 2.5 Let $p^{*} \in C(T)$, then $p_{j}^{*}=0$ for some $j \in T^{-}$implies $z\left(p^{*}\right)=$ $0^{n+1}$.

The simplicial subdivision underlying the $n(n+1)$-ray algorithm to be formulated in Section 2.5, must be such that it subdivides each subset $A(T)$ into $t$-dimensional simplices where $t=|T|-1$ is the dimension of $A(T)$. To describe such a triangulation we firstly introduce for every set $T$ a projection $p(T)$ of $v$ on the intersection of $A(T)$ and the boundary of $S^{n}, b d S^{n}$. For an illustration, we refer to Figure 2.3

Definition 2.6 Let $T$ be as described before, then the projection $\mathrm{p}(\mathrm{T})$ of $\mathbf{v}$ on $\mathbf{A}(\mathbf{T}) \cap \mathbf{b d} \mathbf{S}^{n}$ is given by:

$$
\begin{array}{ll}
p_{j}(T)=0 & \text { if } j \in T^{-}, \\
p_{j}(T)=v_{j} & \text { if } j \in T^{c}, \\
p_{j}(T)=v_{j}\left(\frac{1-\sum_{k \in T^{c}} v_{k}}{\sum_{k \in T^{+}} v_{k}}\right) & \text { if } j \in T^{+} .
\end{array}
$$

With these projections we define the following directions.

Definition 2.7 Let $T$ be as described before and let $t=|T|-1$. Let $T^{0}$ be a subset of $T$, with exactly one positive and exactly one negative element, let $T^{1}=T \backslash T^{0}$, and let $\Gamma\left(T^{\mathrm{1}}\right)=\left(\Gamma_{1}, \ldots, \Gamma_{t-1}\right)$ be a permutation of the $t-1$ elements of $T^{1}$. Then, the directions $q(1), \ldots, q(t)$ are given by

$$
q(h)= \begin{cases}p\left(T^{0}\right)-v & \text { if } h=1, \\ p\left(T^{0} \cup\left\{\Gamma_{1}, \ldots, \Gamma_{h-1}\right\}\right)-p\left(T^{0} \cup\left\{\Gamma_{1}, \ldots, \Gamma_{h-2}\right\}\right) & \text { if } h=2, \ldots, t .\end{cases}
$$




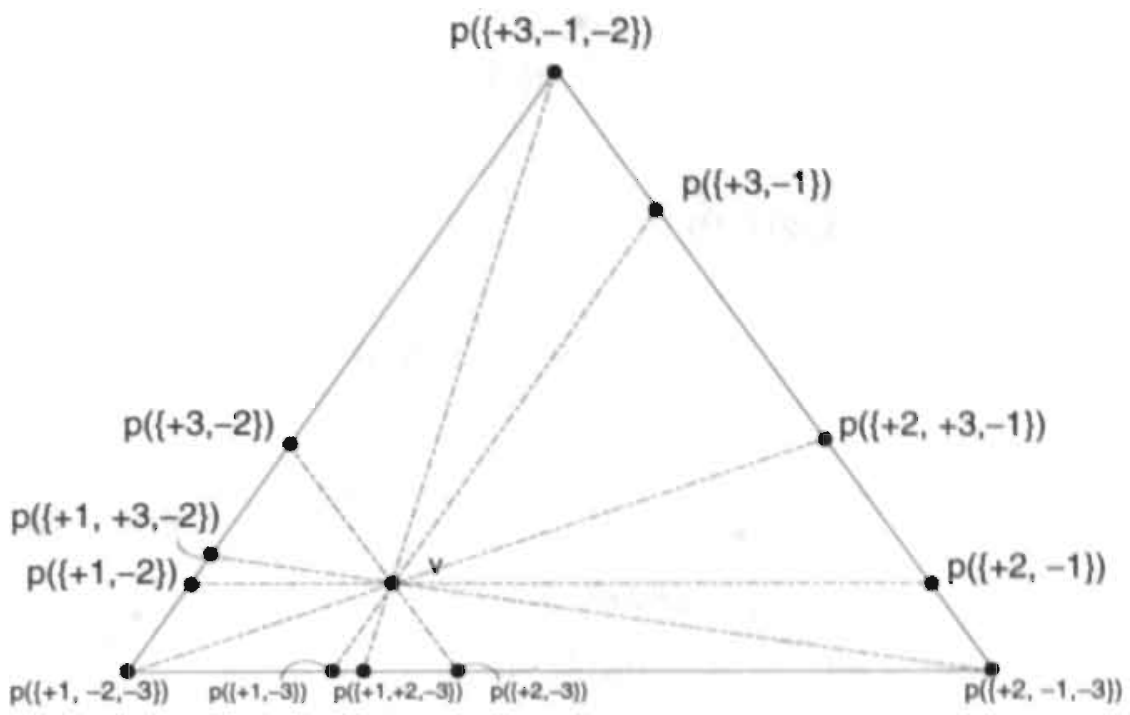

Figure 2.3: Illustration of Definition 2.6: The projections $p(T)$ on the boundaries of $S^{2}$.

With these directions we define the following subsets of the sets $A(T)$, for an illustration see Figure 2.4.

Definition 2.8 Let $T$ be as described before and let $t=|T|-1$. Let $T^{0}$ be a subset of $T$, with exactly one positive and exactly one negative element, let $T^{1}=T \backslash T^{0}$, and let $\Gamma\left(T^{1}\right)=\left(\Gamma_{1}, \ldots, \Gamma_{t-1}\right)$ be a permutation of the $t-1$ elements of $T^{1}$. Then, the set $\mathbf{A}\left(\mathbf{T}^{0}, \boldsymbol{\Gamma}\left(\mathbf{T}^{1}\right)\right)$ is given by:

$$
A\left(T^{0}, \Gamma\left(T^{\ell}\right)\right)=\left\{x \in S^{n} \mid x=v+\sum_{i \in I^{t}} \alpha(i) q(i), 0 \leq \alpha(t) \leq \cdots \leq \alpha(1) \leq 1\right\} .
$$

Observe that for given $T$ with subsets $T^{0}, T^{1}$ as in the preceding definitions, and permutation $\Gamma\left(T^{1}\right)=\left(\Gamma_{1}, \ldots, \Gamma_{t-1}\right)$ of the elements of $T^{1}$, the extreme points of the set $A\left(T^{0}, \Gamma\left(T^{1}\right)\right)$ are precisely the starting point $v$ and the projections $p\left(T^{0}\right), p\left(T^{0} \cup\left\{\Gamma_{1}\right\}\right), \ldots, p\left(T^{0} \cup\left\{\Gamma_{1}, \ldots, \Gamma_{t-1}\right\}\right)$ of $v$ on the boundary of the unit simplex. The directions $q(1), \ldots, q(t)$ are independent by construction. The set $A(T)$ is the union of all. possible sets $A\left(T^{0}, \Gamma\left(T^{1}\right)\right.$ ), 


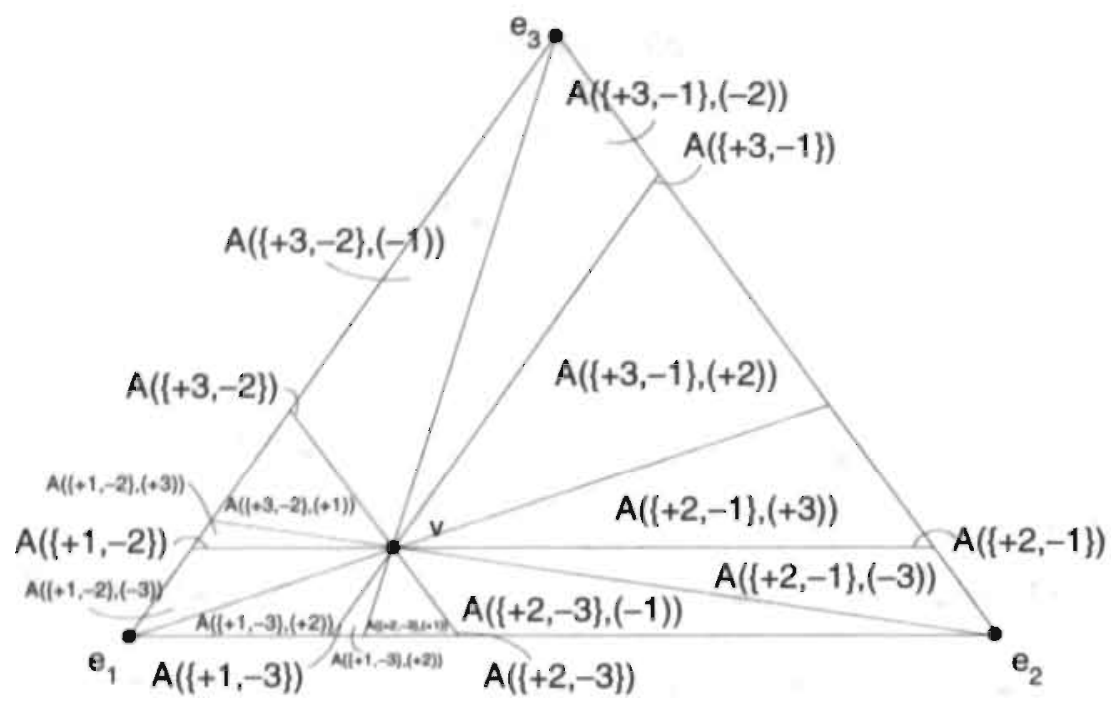

Figure 2.4: Illustration of Definition 2.8: The subsets of the sets $A(T)$.

with $T^{0}, T^{1}$ and $\Gamma\left(T^{1}\right)$ as before, and $A(T)$ is triangulated by subdividing every subset $A\left(T^{0}, \Gamma\left(T^{1}\right)\right)$ of $A(T)$ in $t$-simplices as follows.

Definition 2.9 Let $m$ be a positive integer, and let $T$ and $t$ be given as before. Then, the subset $A\left(T^{0}, \Gamma\left(T^{1}\right)\right)$ of $A(T)$ is subdivided into t-simplices $\sigma(a, \pi)$ with vertices $y^{1}, \ldots, y^{t+1}$ according to the QV-triangulation with grid size $\mathbf{m}^{-1}$ such that:

- $y^{1}=v+m^{-1} \sum_{j \in I^{n+1}} a_{j} q(j)$ with $a=\left(a_{1}, \ldots, a_{n+1}\right)$ an integer vector satisfying $01=a_{n+1}=\ldots=a_{t+1} \leq a_{t} \leq \ldots \leq a_{1} \leq m-1$,

- $y^{i+1}=y^{i}+m^{-1} q\left(\pi_{i}\right)$ for $i=1, \ldots, t$ with $\pi=\left(\pi_{1}, \ldots, \pi_{t}\right)$ a permutation of the $t$ elements of $I^{t}$ such that $p>p^{\prime}$ if $a_{\pi_{p}}=a_{\pi_{p^{\prime}}}$ and $\pi_{p}=k$ and $\pi_{p^{\prime}}=k-1$ for some $k \in\{2, \ldots, t\}$.

The union of the $Q V$-triangulation with grid size $m^{-1}$ of the sets $A\left(T^{0}, \Gamma\left(T^{1}\right)\right)$ where $T^{0}, T^{1}$ and $\Gamma\left(T^{1}\right)$ are for given $T$ as defined before is a simplicial subdivision with grid size $m^{-1}$ of $A(T)$. Let $Q V(m)$ denote the collection 


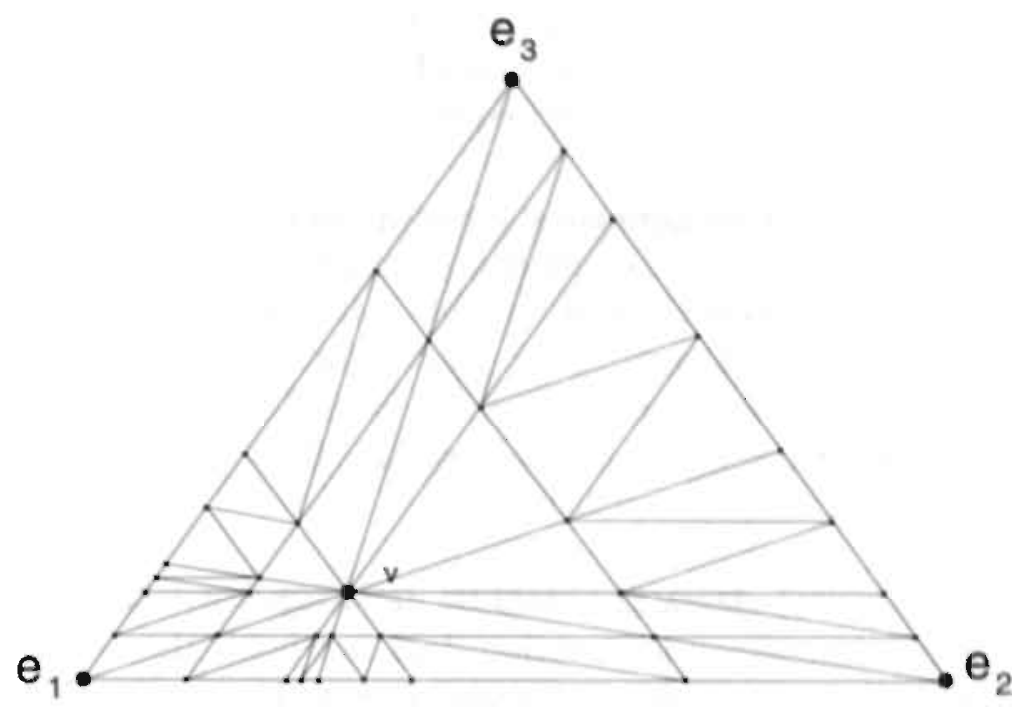

Figure 2.5: Illustration of Definition 2.9: The $Q V$-triangulation of $S^{2}$ for $m=2$.

of simplices of the $Q V$-triangulation with grid size $m^{-1}$. The union of these subdivisions of $A(T)$ over all sets $T$ induces a simplicial subdivision of $S^{n}$, called the $Q V$-triangulation with grid size $m^{-1}$, see Figure 2.5 for $n=2$ and $m=2$.

Definition 2.10 Let $T$ and $t$ be as described before. For $g=t, t-1$, let $\sigma \in Q V(m)$ be a g-simplex with vertices $y^{1}, \ldots, y^{g+1}$ in $S^{n}$, then $\sigma$ is $\mathbf{T}$ complete if the system of linear equations

$\sum_{i \in I^{g+1}} \lambda_{i}\left[\begin{array}{c}z\left(y^{i}\right) \\ 1\end{array}\right]-\sum_{h \in T^{e}} \mu_{h}\left[\begin{array}{c}e_{h} \\ 0\end{array}\right]-\alpha\left[\begin{array}{c}e\left(T^{-}\right) \\ 0\end{array}\right]-\beta\left[\begin{array}{c}e\left(T^{+}\right) \\ 0\end{array}\right]=\left[\begin{array}{c}0^{n+1} \\ 1\end{array}\right]$,

where $e_{h}$ is the $h$-th unit vector in $\Re^{n+1}, e(Q)=\sum_{j \in Q} e_{j}$ for nonempty $Q \subseteq$ $I^{n+1}$, has a solution satisfying $\lambda_{i} \geq 0$ for all $i \in I^{g+1}, \alpha \leq 0, \beta \geq 0$, $\alpha \leq \mu_{h} \leq \beta$ for all $h \in T^{c}$.

Denote $\lambda=\left(\lambda_{1}, \ldots, \lambda_{g+1}\right), \mu=\left(\mu_{h}, h \in T^{c}\right)$. It is easy to see that there are $n+3$ variables if $g=t$ and $n+2$ variables if $g=t-1$. There are $n+2$ linear 
equations to solve in system (2.1). A (feasible) solution $(\lambda, \mu, \alpha, \beta)$ therefore is (in general) not unique if $g=t$. In the latter case a solution $(\lambda, \mu, \alpha, \beta)$. is called a basic solution if at least one of the variables $\alpha, \beta, \lambda_{i}, i \in I^{g+1}$ equals zero or at least one $\mu_{h}, h \in T^{c}$, equals either $\alpha$ or $\beta$.

\section{Assumption 2.1. (Nondegeneracy assumption) Suppose that} $\sigma\left(y_{1}^{1}, \ldots, y^{g+1}\right) \in Q V(m)$ is a $T$-complete $g$-simplex in $A(T)$. If $g=t-1$, then system (2.1) has exactly one feasible solution $(\lambda, \mu, \alpha, \beta)$, satisfying $\alpha \leq 0, \beta \geq 0, \lambda_{j}>0$ for all $j \in I^{t}, \alpha<\mu_{h}<\beta$ for all $h \in T^{c}$. Furthermore, if $g=t$, then at every solution $(\lambda, \mu, \alpha, \beta): \alpha \leq 0, \beta \geq 0$, and if $\alpha \neq 0$, $\beta \neq 0$, then at most one of the inequalities $\lambda_{i} \geq 0$ for $i \in I^{g+1}, \alpha \leq \mu_{h} \leq \beta$ for all $h \in T^{c}$, holds with an equality.

Generically, the linear system (2.1) will be nondegenerate. However, if at a solution this nondegeneracy assumption does not hold, then one may perform pivot steps in lexicographic order analogous to Herings [1995] and Yang [1996]. For a $T$-complete $t$-simplex $\sigma(a, \pi)$ with vertices $y^{1}, \cdots, y^{t+1}$ in a given $A(T)$, the set of solutions of system (2.1) determines under Assumption 2.1 a line segment of solutions $(\lambda, \mu, \alpha, \beta)$. A line segment of solutions induces a linear piece of points $p=\sum_{i \in I^{t+1}} \lambda_{i} y^{i}$ in $\sigma(a, \pi)$. This linear piece. has two different end points corresponding with the two basic solutions of system (2.1). This linear piece of points in $\sigma(a, \pi)$ can be followed by making a linear programming pivoting step in system (2.1) above with the variable that is binding at one of the basic solutions.

Definition 2.11 Let $\sigma(a, \pi)=\sigma\left(y^{1}, \ldots, y^{t+1}\right) \in Q V(m)$ be at-simplex. Then, the piecewise linear approximation $\mathrm{Z}$ of $\mathrm{z}$ with respect to $\sigma(a, \pi)$ is given by

$$
Z(p)=\sum_{i \in I^{2+1}} \theta_{i} z\left(y^{i}\right)
$$

where $p=\sum_{i \in I^{t+1}} \theta_{i} y^{i} \in \sigma(a, \pi), \sum_{i \in I^{t+1}} \theta_{i}=1, \theta_{i} \geq 0$ for all $i \in I^{t+1}$.

The $Q V$-triangulation simplicially subdivides the entire unit simplex, so every point in the unit simplex belongs to a simplex as described in the definition above. So, we may define the linear approximation $Z: S^{n} \rightarrow \Re^{n+1}$ of $z: S^{n} \rightarrow \Re^{n+1}$ with respect to the $Q V$-triangulation, pointwise by Definition 2.11. Clearly $\sigma(a, \pi)$ is a $T$-complete $t$-simplex in $A(T)$ with solution 
$(\lambda, \mu, \alpha, \beta)$ of the system (2.1), if and only if for $p=\sum_{i \in I^{t+1}} \lambda_{i} y^{i} \in \sigma(a, \pi)$ :

$$
\begin{array}{lll}
Z_{i}(p)=\alpha & \text { and } p_{i} \leq v_{i} \text { if } i \in T^{-}, \\
\alpha \leq Z_{i}(p)=\mu_{i} \leq \beta & \text { and } p_{i}=v_{i} \text { if } i \in T^{c}, \\
Z_{i}(p)=\beta & \text { and } p_{i} \geq v_{i} \text { if } i \in T^{+} .
\end{array}
$$

As a measure how close the piecewise linear approximation $Z$ of $z$ approximates the real excess demand function $z$, one may use

$$
\max _{\sigma \in Q V(m)} \max _{p \in \sigma} d_{2}(Z(p), z(p)) .
$$

Clearly, $\lim _{m \rightarrow \infty} \max _{\sigma \in Q V(m)} \max _{p \in \sigma} d_{2}(Z(p), z(p)) \rightarrow 0$.

\subsection{Paths of price adjustment processes}

In Walras' so-called successive tâtonnement process (Walras [1874]), an 'auctioneer' is appointed. The auctioneer calls out prices for all commodities, and all agents respond by giving their excess demands for all commodities at these prices. In general, the markets of all goods will not be in equilibrium. To reach an equilibrium, the auctioneer changes the price of the first good in such a manner that the excess demand of this good becomes equal to zero. The auctioneer proceeds with the second good to reach an equilibrium in the market for the second good, and so forth. When the market for the last commodity is in equilibrium, the auctioneer may have to repeat the whole procedure over again. In general namely, the markets of the other goods will not be in equilibriim any more. The procedure stops whenever the excess demand of each commodity is zero or close enough to zero. An unatiractive feature of this process is that the auctioneer ignores information from the markets of all other goods, while adjusting the price of a certain good in order to reach an equilibrium on that market.

Samuelson [1947] formulated the so-called simultaneous tâtonnement process. Given an excess demand function $z: \Re_{+}^{n+1} \rightarrow \Re^{n+1}$, the simultaneous tâtonnement process is given by:

$$
\frac{d p}{d t}=z(p) \text { for all } p \in \Re_{+}^{n+1} \backslash\left\{0^{n+1}\right\} .
$$

Since the excess demand function $z$ is continuous, there exists a solution curve $\{p(t)\}_{t \geq 0}$ satisfying $p(0)=p_{0} \in \Re_{+}^{n+1} \backslash\left\{0^{n+1}\right\}$ and Equation (2.3). By using 
Walras' law, i.e., $p \cdot z(p)=0$ for all $p \in \Re_{+}^{n+1} \backslash\left\{0^{n+1}\right\}$, it may be confirmed that on the solution curve $\{p(t)\}_{t \geq 0}$ the length (measured in the Euclidean norm) of the vectors being generated under this process, remains constant.

The interpretation of such a solution curve, i.e., a path of points representing price vectors, generated. by Samuelson's process is intuitively and. economically appealing. Under the simultaneous tâtonnement process at any price vector on such a path, the price of each good with positive excess demand is increased, the price of every good with zero excess demand remains unchanged, and the price of each good with negative excess demand. is decreased. Furthermore, the changes in the prices of the commodities are proportional to their excess demands. We refer to Arrow \& Hurwicz [1958], Arrow et al. [1959], Uzawa [1961] for sufficient conditions under which a large class of price adjustment processes converge. These conditions are generally viewed as quite strong, and certainly not all economies satisfy these conditions. For some time attention in the profession focused on finding weaker conditions on excess demand functions and price adjustment processes for which convergence to an equilibrium price vector was guaranteed. Among these conditions, conditions for uniqueness of an equilibrium price vector received particular interest. Scarf [1960] showed conclusively that the simultaneous tâtonnement process may not converge to an equilibrium price vector, even if this equilibrium price vector is uniquely determined.

The results of Sonnenschein [1972, 1973], Mantel [1974], and Debreu [1974] imply that an excess demand function for an exchange economy is characterized by continuity, Walras' law, and homogeneity of degree zero in the prices. Every exchange economy with utility maximizing agents, yields an excess demand function fulfilling these three conditions. Moreover, for any continuous function satisfying Walras' law and homogeneity of degree zero in the prices, an exchange economy with utility maximizing agents can be constructed, such that the resulting excess demand function is equal to the former function on an arbitrarily large subset of the interior of the space of all possible price vectors. So there is no set of conditions on excess demand functions guaranteeing convergence of Samuelson's simultaneous tâtonnement process to an equilibrium price vector which will hold in general.

Smale [1976] formulated a more sophisticated method of price adjustment, called the Global Newton method. This method has the form, for $t \geq 0$,

$$
p_{n+1}(t)=1 \text {, and }
$$




$$
z_{j}(p(t))=\lambda(t) z_{j}(p(0)), j=1, \ldots, n
$$

where $z$ is a continuously differentiable excess demand function. The scalar $\lambda(t)$ is a real valued function evaluated at point $t$ in time. For almost all initial price vectors $p(0)$ on the boundary of $\Re_{+}^{n+1} \backslash\left\{0^{n+1}\right\}$ with $p_{n+1}(0)=1$, the Global Newton method converges to an equilibrium price vector, provided the eigenvalues of the $(n \times n)$-Jacobian-matrix $D z(p)$ of the function $\left(z_{1}, \ldots, z_{n}\right)$ are all nonzero at a zero of $z$. Equations (2.4) can be rewritten as

$$
\frac{d z_{j}(p)}{d t}=-\lambda(t) z_{j}(p), j=1, \ldots, n \text {. }
$$

Equation (2.5) implies that under this price adjustment process the change in the excess demands of each of the first $n$ commodities, is proportional to the excess demand itself, and has the opposite sign. Note furthermore that the excess demand of the first $n$ commodities are maximal throughout the entire process.

A price adjustment process is said to be universally convergent if it converges to an equilibrium price vector for (almost) all aggregate excess demand functions. If the process additionally accomplishes this feat while being started in an (almost) arbitrary price vector, then it is called globally convergent. We call a process effective in the sense of Saari \& Simon [1978], if the solutions converge to an equilibrium price vector for (almost) all initial price vectors in some subset of the space on which the excess demand is given. Keenan [1981] calls a process locally effective if convergence to an equilibrium price vector is guaranteed if the process is started sufficiently near an equilibrium price vector. By the above it should be clear that Walras' successive tâtonnement process is not universally convergent, neither is Samuelson's simultaneous tâtonnement process.

Keenan [1981] showed that if the Global Newton method is started in the interior of the price space, sufficiently near an equilibrium price vector, convergence of the Global Newton method is still guaranteed. Hence, Smale's computational process is universally convergent, effective and even locally effective. However, for an arbitrary interior starting point Keenan [1981] showed that convergence to an equilibrium price vector is not guaranteed.

Under Scarf's computational process [1967], when started at the vertex $e_{n+1}=(0, \ldots, 0,1)^{\top}$ of the $n$-dimensional unit simplex, a path of prices on the unit simplex is followed, satisfying:

$$
z_{j}(p)=z_{k}(p) \text { for all } j, k \in I^{n+1} \text {, such that } j, k \neq n+1,
$$


for all prices $p$ of the path. Hence, the changes in the first $n$ components of the excess demand function $z$ are proportional to $z$ itself in this process as well. Several authors have remarked that Smale's Global Newton method follows the same path as Scarf's computational process when the former is started at the price vector $(0, \ldots, 0,1)^{\top}$. An alternative manner of describing $S$, i.e., the path of points $p$ followed by the processes of Scarf and Smale when started at $v=(0, \ldots, 0,1)^{\top}$, is

$$
\begin{array}{ll}
p_{j} \geq v_{j} & \text { and } \quad z_{j}(p)=\max _{h \in I^{n+1}} z_{h}(p), j=1, \ldots, n, \\
p_{n+1} \leq v_{n+1} & \text { and } \quad z_{n+1}(p)=\min _{h \in I^{n+1}} z_{h}(p) .
\end{array}
$$

So, the prices of the first $n$ commodities are increased from their initial levels, and the excess demand of these commodities are kept maximal and equal to each other. The price of the last commodity is decreased from its initial level and its excess demand is kept minimal. The path of the Global Newton method, and the path of Scarf's process when started at the point $e_{3}$, are illustrated in Figure 2.6.

We now describe the path followed by our price adjustment process to be presented in Section 2.4. This path is approximately followed by the $n(n+1)$ ray algorithm to be presented in Section 2.5. Let $v$ be an arbitrary point $S^{n}$. The price adjustment process to be presented in Section 2.4 of this chapter, when started at $v$, follows a path of points in some set $A(T)$ for varying $T$ such that for each point $p$ on a curve in $A(T)$ it holds that

$$
\begin{array}{lll}
p_{j} \leq v_{j} & \text { and } z_{j}(p)=\min _{h \in I^{n+1}} z_{h}(p) & \text { for all } j \in T^{-}, \\
p_{j}=v_{j} & \text { and } \min _{h \in I^{n+1}} z_{h}(p) \leq z_{j}(p) \leq \max _{h \in I^{n+1}} z_{h}(p) & \text { for all } j \in T^{c}, \\
p_{j} \geq v_{j} & \text { and } z_{j}(p)=\max _{h \in I^{n+1}} z_{h}(p) & \text { for all } j \in T^{+} .
\end{array}
$$

Following Herings [1994], we may assume without loss of generality that the set of points in $S^{n}$ satisfying (2.6) consists of loops and paths. Exactly one path connects the starting point $v$ with a solution to the zero point problem, i.e., a vector $p^{\prime}$ satisfying $z\left(p^{\prime}\right)=0^{n+1}$. This path, to be denoted by $P$, can be interpreted in economic terms as follows. Along the first (linear) piece of the path the price of the commodity with highest excess demand is increased and the price of the commodity with lowest excess demand is simultaneously decreased by the same amount, while the prices of all commodities having neither maximal nor minimal excess demand are not changed.

In general, along the path the price of a commodity is increased from. its initial price value when its excess demand becomes maximal, and it is 


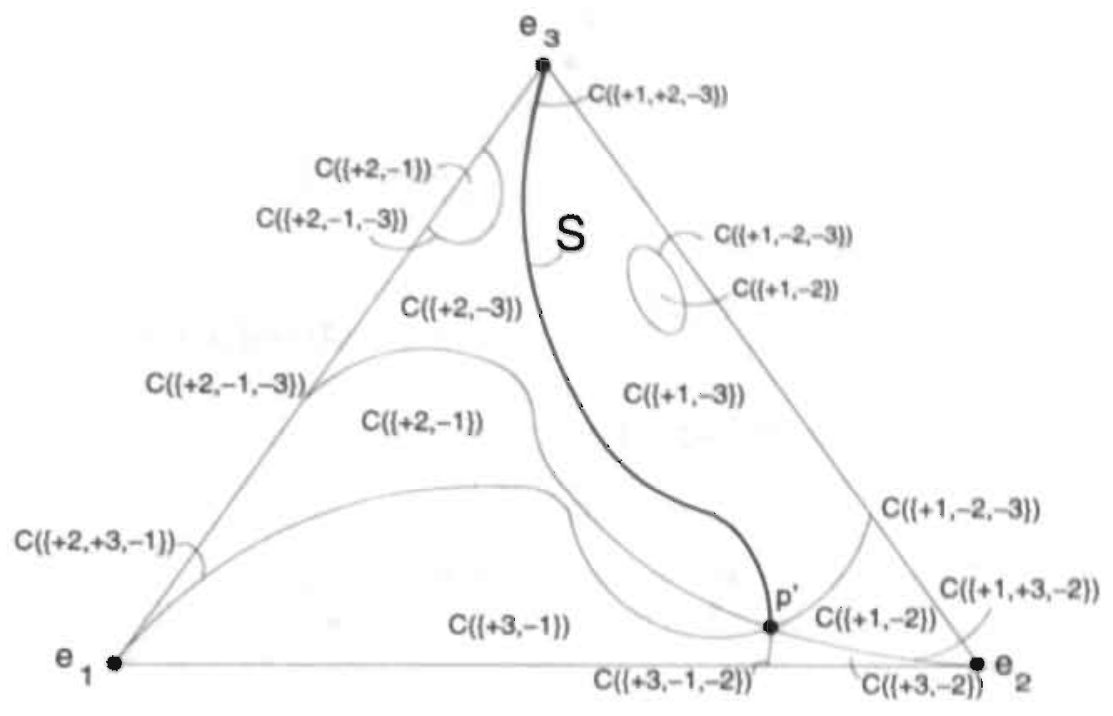

Figure 2.6: The path $S$ followed by Smale's Global Newton method, and by Scarf's process provided the latter is started with the price of commodity 3 equal to one. The path ends at $p$.

decreased from its initial price value when its excess demand becomes minimal. Meanwhile, the prices of the commodities having neither minimal, nor maximal excess demand are kept equal to the initial prices. If however, along the path the price of a commodity which has been raised (lowered) from its starting value becomes equal to this initial value again, then it is kept equal to this initial price value, and the excess demand is allowed to decrease from the maximal value (to increase from the minimal value). We refer to. Figure 2.7 for an graphic illustration for the example formed by Figures 2.1 and 2.2.

In Section 2.5 we will present the so-called $n(n+1)$-ray variable dimension restart algorithm. This algorithm, when started at $v$, approximately follows the path $P$, and terminates at an approximation of an equilibrium price vector. Given the $Q V$-triangulation with some grid size $m^{-1}$, a piecewise linear path of points in a sequence of adjacent $T$-complete $t$-simplices $\sigma(a, \pi)$ in $A(T)$ with $T$-complete common facets, for varying sets $T$, is generated. Each simplex of the sequence contains a line segment as piece of the path fulfilling. 


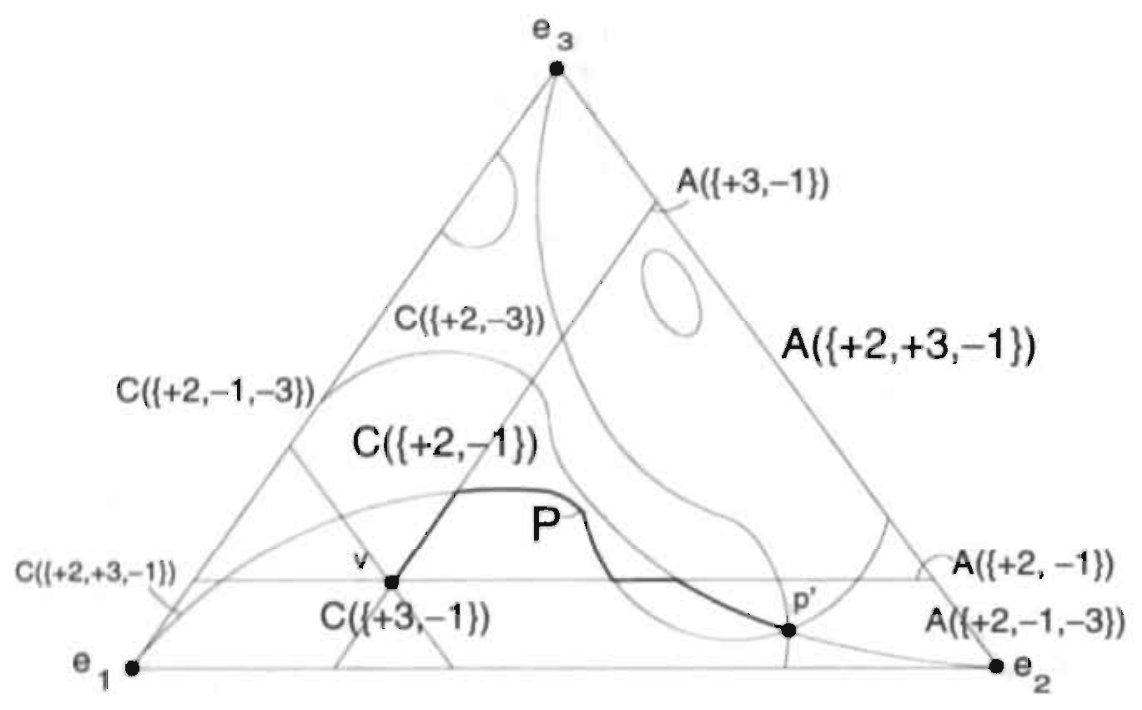

Figure 2.7: The path $P$ followed by our price adjustment process, and approximately followed by our $n(n+1)$-ray algorithm. The path $P$ connects the point $v$ and the equilibrium price vector $p l$.

(2.2), i.e., it satisfies (2.6) with the excess demand function $z$ replaced by its piecewise linear approximation $Z$. The line segment in such a simplex may be followed by making a linear programming pivot step in the linear system (2.1). How closely the piecewise linear path generated by the $n(n+1)$-ray algorithm follows the path $P$, depends crucially on the grid size of the triangulation. The smaller the grid size $m^{-1}$ of the $Q V$-triangulation namely, the better $Z$ approximates $z$, and the more accurately the algorithm will follow the path $P$.

\subsection{Globally convergent price adjustment}

The price adjustment process may start at an arbitrary point of the unit simplex. If the starting point $v \in S^{n}$, is not an equilibrium price vector, we may assume, following Herings [1994], that there is precisely one good, say $i^{*}$, with maximal positive excess demand, and there is precisely one other 
good, say $j^{*} \in \operatorname{Car}(v)=\left\{j \in I^{n+1} \mid v_{j}>0\right\}$, with minimal negative excess demand. Hence, $v \in C\left(\left\{+i^{*},-j^{*}\right\}\right)$. Then, under the price adjustment process, initially $p_{i^{*}}$, the price of commodity $i^{*}$, is increased from $v_{i *}$, and simultaneously $p_{j^{*}}$, the price of commodity $j^{*}$, is decreased from $v_{j^{*}}$. The prices of all other commodities remain unchanged initially.

Throughout the entire process, we make the following nondegeneracy assumption: At any point in $S^{n}$ reached by the price adjustment process which is not an equilibrium price vector, it holds that for at most one commodity the excess demand becomes maximal or minimal, or its price becomes equal to the initial value again. This nondegeneracy assumption is made for convenience in the ensuing description, and it holds generically.

The price adjustment process leaves the starting point $v$, along the ray formed by the one-dimensional set $A\left(\left\{+i^{*},-j^{*}\right\}\right)$ by increasing the price of good $i^{*}$ from the initial level $v_{i^{*}}$, decreasing the price of good $j^{*}$ from the initial level $v_{j^{*}}$, and keeping the prices of all other commodities fixed at their initial levels. One of the following three mutually exclusive situations will occur along $A\left(\left\{+i^{*},-j^{*}\right\}\right)$ :

1. For some price vector $p^{*}$ it holds that $p_{j}^{*}=0$;

2. For some price vector $p^{*}$ it holds that the excess demand of some commodity, say $i^{\prime} \in T^{c}$, becomes equal to the excess demand of commodity $i^{*}$

3. For some price vector $p^{*}$ it holds that the excess demand of some commodity, say $j^{\prime} \in T^{c}$, becomes equal to the excess demand of commodity $j^{*}$.

If Situation 1 occurs, then the price adjustment process stops at $p^{*}$. Since $j^{*} \in T^{-} z\left(p^{*}\right)=0^{n+1}$ by Lemma 2.5 .

If Situation 2 occurs, then the excess demand of commodity $i^{\prime}$ becomes maximal. The process continues by raising the price of commodity $i^{\prime}$, from its initial level $v_{i^{\prime}}$, and by keeping the excess demands of commodities $i^{*}$ and $i^{\prime}$, respectively, equal to each other. Thus, the set $A\left(\left\{+i^{*},+i^{\prime},-j^{*}\right\}\right)$ of $S^{n}$ is entered and a path of points in this set is followed satisfying $z_{i} \cdot(p)=z_{i^{\prime}}(p)$ for each point $p$ of the path.

If Situation 3 occurs, then the excess demand of commodity $j^{\prime}$ becomes minimal. The process continues by lowering the price of commodity $j^{\prime}$, from its initial level $v_{j^{\prime}}$, and by keeping $z_{j^{*}}$ and $z_{j^{\prime}}$, the excess demands 
of commodities $j^{*}$ and $j^{\prime}$, respectively, equal to each other. Thus, the set $A\left(\left\{+i^{*},-j^{*},-j^{\prime}\right\}\right)$ of $S^{n}$ is entered and a path of points in this set is followed satisfying $z_{j^{*}}(p)=z_{j^{\prime}}(p)$ for each point $p$ of the path.

In general, the price adjustment process traces a path in some set $A(T)$ for varying $T$ satisfying for every $p$ on a curve in $A(T)$ that

$$
\begin{array}{ll}
p_{j} \leq v_{j} \text { and } z_{j}(p)=\min _{h \in I^{n+1}} z_{h}(p) & \text { for all } j \in T^{-}, \\
p_{j}=v_{j} \text { and } \min _{h \in I^{n+1}} z_{h}(p) \leq z_{j}(p) \leq \max _{h \in I^{n+1}} z_{h}(p) & \text { for all } j \in T^{c}, \\
p_{j} \geq v_{j} \text { and } z_{j}(p)=\max _{h \in I^{n+1}} z_{h}(p) & \text { for all } j \in T^{+} .
\end{array}
$$

Generically, following the proof of Theorem 10.4.2 of Herings [1995], the set of points satisfying (2.6) for some set $T$ consists of a finite number of curves, each curve being a loop or a path with two end points. Then, one of the following cases may occur at an end point of a path in $A(T)$ :

1. The price adjustment process reaches a point $p^{*} \in b d(A(T))$;

2. The price adjustment process reaches a point $p^{*} \in \operatorname{int}(A(T))$ such that for some $j^{*} \in T^{c}$ it holds that $z_{j^{*}}\left(p^{*}\right)$ either becomes maximal or becomes minimal;

3. The price adjustment process reaches a point $p^{*} \in \operatorname{int}(A(T))$ satisfying $\min _{h \in I^{n+1}} z_{h}\left(p^{*}\right)=\max _{h \in I^{n+1}} z_{h}\left(p^{*}\right)$.

In Case $1, p^{*}$ satisfies $p_{j}^{*}=0$ for some $j \in T^{-}$, or $p^{*} \in A(K)$ for some nonempty set $K \subset T$, satisfying $|T \backslash K|=1$. In the first subcase of Case 1 , the price adjustment process stops at $p^{*}$. It follows namely by Lemma 2.5 that $p^{*}$ is an equilibrium price vector. In the second subcase of Case 1 , $p_{k}^{*}=v_{k}$ for precisely one $k \in I^{n+1} \backslash T^{c}$. Let $K:=T \backslash\{k\}$ if $k \in T^{+}$, and $K:=T \backslash\{-k\}$ if $k \in T^{-}$, then the price adjustment process continues by tracing a path of points $p$ in $A(K)$, satisfying (2.6) with $T$ equal to $K$.

In Case 2, the price adjustment process continues by tracing a path of points $p$ in the set $A(K)$, where $K:=T \cup\left\{+j^{*}\right\}$ if $z_{j^{*}}\left(p^{*}\right)=\max _{h \in I^{n+1}} z_{h}\left(p^{*}\right)$, and $K:=T \cup\left\{-j^{*}\right\}$ if $z_{j^{*}}\left(p^{*}\right)=\min _{h \in I^{n+1}} z_{h}\left(p^{*}\right)$, satisfying (2.6) with $T$ equal to $K$.

In Case 3, it follows from Lemma 2.4 that $p^{*}$ is an equilibrium price vector, and the price adjustment process terminates.

In this manner, starting at $v$, the price adjustment process follows a path of points, corresponding with price vectors, in sets of which the dimension 
may vary between 1 and $n$, for an exchange economy with $n+1$ commodities. Such a set $A(T)$ is completely determined by the arbitrarily chosen starting point of the process, $v \in S^{n}$, and a certain set of positive and negative integers $T \subset I^{n+1} \cup-I^{n+1}$, with $\operatorname{dim} A(T)$ equal to $|T|-1$. Along the path followed by the process in such a set $A(T)$, the excess demand of any commodity with index $k \in T \cap I^{n+1}$ is kept maximal, and the price of any such commodity may be higher than the initial level. Furthermore, the excess demand of any commodity with index $k \in T \cap-I^{n+1}$ is kept minimal, and the price of such a commodity may be lower than the initial level. The prices of all other commodities in the exchange economy are kept equal to their initial levels, and the excess demands of these commodities are allowed to vary between the levels of minimal and maximal excess demand.

If the excess demand of any of the latter commodities becomes equal to either maximal or minimal excess demand as well, then the price adjustment process continues in a set of dimension. one higher. On the other hand, as soon as the price of a commodity becomes equal to its initial level again, while having been raised or lowered from its initial value, the price adjustment process continues in a set of one dimension lower. The path of points followed by the price adjustment process, connects the starting point $v$ with a point representing an equilibrium price vector. The price adjustment process terminates at an equilibrium price vector for arbitrary excess demand functions under precisely the conditions which guarantee the existence of at least one equilibrium price vector, while being started at an arbitrary price vector. The informational requirements for this globally convergent price adjustment process consist of local information obtained from the excess demand function and global information about the location of the current price vector in relation to the starting point $v$.

The remainder of this subsection is devoted to a detailed discussion of the examples in Figures 2.1 and 2.2 as depicted in Figure 2.8. At the starting point $v$, the excess demand of commodity 3 is maximal and the excess demand of commodity 1 is minimal, since $v \in C(\{+3,-1\})$. The price adjustment process leaves the starting point $v$ by raising the price of commodity 3 and simultaneously lowering the price of the commodity 1 from their respective initial levels $v_{1}$ and $v_{3}$. Thus, the one-dimensional set $A(\{+3,-1\})$ is entered 


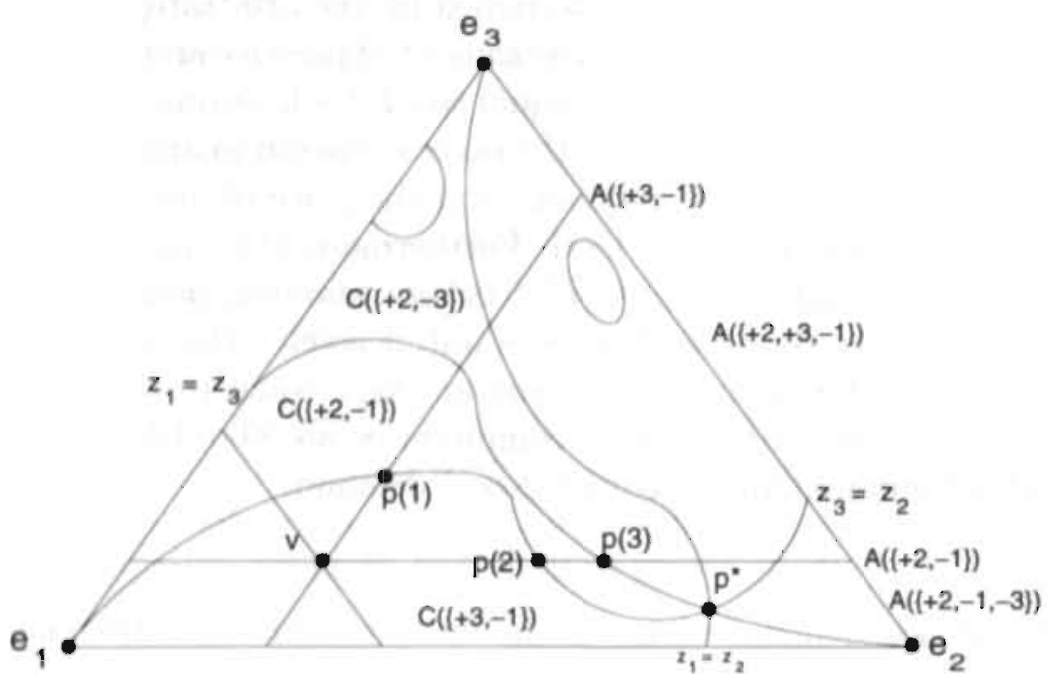

Figure 2.8: The price adjustment process follows the path $P$ by moving from the starting point $v$ via $p(1), p(2)$ and $p(3)$, to the equilibrium price vector $p^{*}$. The actions taken at these points necessary to follow the path $P$ are described in the example.

and a curve of points $p$ in $S^{2}$ is traced, satisfying

$$
\begin{aligned}
& p_{3} \geq v_{3} \text { and } z_{3}(p)=\max _{h \in I^{3}} z_{h}(p) \text {, } \\
& p_{2}=v_{2} \text { and } z_{1}(p) \leq z_{2}(p) \leq z_{3}(p) \text {, } \\
& p_{1} \leq v_{1} \text { and } z_{1}(p)=\min _{h \in I^{3}} z_{h}(p) \text {, }
\end{aligned}
$$

until $p(1)$ is reached. At the price vector $p(1)$, it holds that $z_{2}(p(1))$, the excess demand of commodity 2 , is equal to $z_{3}(p(1))$, the excess demand of commodity 3 . Moreover, $z_{2}(p(1))$ and $z_{3}(p(1))$ are both maximal. The process continues by raising the price of commodity 2 from its initial level $v_{2}$, and by keeping $z_{2}$ and $z_{3}$ equal to each other. Thus, the set $A(\{+2,+3,-1\})$ is entered and a curve of points $p$ in $S^{2}$ is traced, satisfying

$$
\begin{aligned}
& p_{j} \geq v_{j} \quad \text { and } z_{j}(p)=\max _{h \in I^{3}} z_{h}(p) \text { for } j \in\{2,3\}, \\
& p_{1} \leq v_{1} \text { and } z_{1}(p)=\min _{h \in I^{3}} z_{h}(p),
\end{aligned}
$$

until $p(2)$ is reached. At the price vector $p(2)$, the price of commodity 3 
which had initially been raised, has become equal to its initial level $v_{3}$ again. Then, the process continues by keeping the price of commodity 3 at $v_{3}$, and by allowing the excess demand of commodity $3, z_{3}(p)$, to become less than the maximal excess demand, $z_{2}(p)$. The latter causes a raise of the price of commodity 2 and simultaneously a lowering of the price of commodity 1 . Hence, the process proceeds by entering the one-dimensional set $A(\{+2,-1\})$ and following a curve of points $p$ in $S^{2}$ satisfying

$$
\begin{aligned}
& p_{2} \geq v_{2} \text { and } z_{2}(p)=\max _{h \in I^{3}} z_{h}(p) \text {, } \\
& p_{3}=v_{3} \text { and } z_{1}(p) \leq z_{3}(p) \leq z_{2}(p) \text {, } \\
& p_{1} \leq v_{1} \text { and } z_{1}(p)=\min _{h \in I^{3}} z_{h}(p) \text {, }
\end{aligned}
$$

until $p(3)$ is reached. At this price vector the excess demand of commodity 3 becomes equal to the minimal excess demand of commodity 1 . The process then proceeds by lowering the price of commodity 3 from $v_{3}$ and keeping $z_{1}$ equal to $z_{3}$. Thus, the set $A(\{+2,-1,-3\})$ is entered and a curve of points $p$ in $S^{2}$ is followed satisfying

$$
\begin{aligned}
& p_{2} \geq v_{2} \text { and } z_{2}(p)=\max _{h \in I^{3}} z_{h}(p), \\
& p_{2} \leq v_{2} \text { and } z_{j}(p)=\min _{h \in I^{3}} z_{h}(p) \text { for } j=1,3,
\end{aligned}
$$

until $p^{*}$ is reached. At the price vector $p^{*}$, it holds that $z_{1}\left(p^{*}\right)=z_{2}\left(p^{*}\right)=$ $z_{3}\left(p^{*}\right)$, hence $p^{*}$ is an equilibrium price vector by Lemma 2.4 .

\subsection{A globally convergent algorithm}

\subsubsection{Description of the algorithm}

The algorithm may start in an arbitrary interior point $v$ of the unit simplex. If $v$ is not an equilibrium price vector, we may assume without loss of generality by the results of Herings [1994] that there is a unique maximal component of $z(v)$, say $i$, and a unique minimal component of $z(v)$, say $j$. Then, according to (2.6) with $z$ replaced by its piecewise linear approximation $Z$ with respect to the $Q V$-triangulation with some grid size $m^{-1}$, the algorithm leaves $v$ by increasing $p_{i}$ and by decreasing $p_{j}$ by the same amount, along the onedimensional set $A(\{+i,-j\})$.

The algorithm will generate a path of connected line segments of points $p$ satisfying (2.2) contained in a sequence of adjacent $T$-complete $t$-simplices $\sigma(a, \pi)$ having $T$-complete common facets in sets $A(T)$ of varying sets $T$, by 
going for each simplex in the sequence from one basic solution of system (2.1) to the other one. This is accomplished by performing a linear programming pivot step in (2.1) with the variable that is binding at the first basic solution.

For the set of solutions $(\lambda, \mu, \alpha, \beta)$ of system (2.1) with respect to a $T$ complete $t$-simplex $\sigma(a, \pi)$ of the $Q V$-triangulation with grid size $m^{-1}$ in a given set $A\left(T^{0}, \Gamma\left(T^{1}\right)\right)$, the line segment of points $p=\sum_{j=1}^{t+1} \lambda_{j} y^{j}$ connects exactly two points corresponding with the two basic solutions. Each point $p$ on this line segment satisfies (2.2). If $\sigma(a, \pi)$ is generated by the algorithm, it moves in $\sigma(a, \pi)$ from one point corresponding with a basic solution, say $p^{1}$, to the other one, say $p^{2}$. Whenever $\alpha=0$ or $\beta=0$, then the round of the algorithm terminates with solution $\left(\lambda^{*}, \mu^{*}, \alpha^{*}, \beta^{*}\right)$ with $p^{*}=\sum_{k=1}^{t+1} \lambda_{k}^{*} y^{k}=p^{2}$ as an approximating solution of the zero point problem of $z$. Otherwise, $\alpha<0<\beta$ and one of the following two situations may occur:

1. $p^{2}$ is an element of a $T$-complete $(t-1)$-facet of $\sigma(a, \pi)$ and due to Assumption 2.1 exactly one $\lambda_{i}, i \in I^{t+1}$, equals zero,

2. $p^{2}$ is in the interior of $\sigma(a, \pi)$ and due to Assumption 2.1, exactly one $\mu_{h}, h \in T^{c}$, equals either $\alpha$ or $\beta$.

In Situation 1 the algorithm distinguishes two cases. Let $\lambda_{s}=0, s \in I^{t+1}$, at $p^{2}$, then $p^{2}$ is a point in the $T$-complete $t$-facet $\tau$ opposite vertex $y^{s}$, and:

la. $\tau$ lies in the interior of $A\left(T^{0}, \Gamma\left(T^{1}\right)\right)$, or

1b. $\tau$ lies in the boundary of $A\left(T^{0}, \Gamma\left(T^{1}\right)\right)$.

In Case 1a, the algorithm moves from $\sigma(a, \pi)$ to the unique $T$-complete $t$-simplex $\sigma(\underline{a}, \underline{\pi})$ in $A\left(T^{0}, \Gamma\left(T^{1}\right)\right)$ which shares the facet $\tau$ with $\sigma(a, \pi)$. The parameters $\underline{a}$ and $\underline{\pi}$ of the new simplex $\sigma(\underline{a}, \underline{\pi})$ are given in Table 2.1. By making the adaptations described, a new vertex is generated, say $\underline{y}^{s^{\prime}}$, for some $s^{\prime} \in I^{t+1}$. This new vertex of $\sigma(\underline{a}, \underline{\pi})$ lies opposite $\tau$ and replaces $y^{s}$ in system (2.1). Next a linear programming pivot step is performed in system (2.1) with $\left[\begin{array}{c}z\left(\underline{y}^{s^{\prime}}\right) \\ 1\end{array}\right]$ by increasing $\lambda_{s^{\prime}}$ from zero. Thus a line segment in $\sigma(\underline{a}, \underline{\pi})$ is generated. 


\begin{tabular}{|l|l|l|}
\hline$s$ & $\underline{\pi}$ & $\underline{a}$ \\
\hline$s=1$ & $\left(\pi_{2}, \pi_{\left.3, \ldots, \pi_{t}, \pi_{1}\right)}\right.$ & $a+e\left(\pi_{1}\right)$ \\
\hline $2 \leq s \leq t$ & $\left(\pi_{1}, \ldots, \pi_{s-2}, \pi_{s}, \pi_{s-1}, \pi_{s+1}, \ldots, \pi_{t}\right)$ & $a$ \\
\hline$s=t+1$ & $\left(\pi_{t}, \pi_{1}, \pi_{2}, \ldots, \pi_{t-1}\right)$ & $a-e\left(\pi_{t}\right)$ \\
\hline
\end{tabular}

Table 2.1. Given $\sigma(a, \pi)$ with parameters $a=\left(a_{1}, \ldots, a_{n+1}\right)$ and $\pi=$ $\left(\pi_{1}, \ldots, \pi_{t}\right), \underline{a}$ and $\underline{\pi}$ are the parameters of the $t$-simplex $\sigma(\underline{a}, \underline{\pi})$ sharing the facet $\tau$ opposite the vertex $y^{s}$ with $\sigma(a, \pi)$.

In Case 1b, the algorithm distinguishes three possibilities:

1. $s=1, \pi_{1}=1$, and $a_{\pi_{1}}=m-1$,

2. $2 \leq s \leq t, a_{\pi_{s-1}}=a_{\pi_{s}}, \pi_{s-1}=k-1$ and $\pi_{s}=k$ for a certain $k$, $2 \leq k \leq t$,

3. $s=t+1, \pi_{t}=t, a_{\pi_{t}}=0$.

In Possibility 1, the facet $\tau$ lies opposite vertex $y^{1}$ in $b d S^{n}$. Therefore, for some $i \in T^{-}$it holds that $p_{i}=0$ for every $p \in \tau$. This implies $y_{i}^{j}=0$ and hence $z_{i}\left(y^{j}\right) \geq 0$ for all $j=2, \ldots, t+1$. Let at $p^{2}=p^{*}$, the basic solution be given by $\left(\lambda^{*}, \mu^{*}, \alpha^{*}, \beta^{*}\right)$, i.e., $p^{*}=\sum_{j=2}^{t+1} \lambda_{j}^{*} y^{j}$. Clearly $Z\left(p^{*}\right)=\sum_{j=2}^{t+1} \lambda_{j}^{*} z\left(y^{j}\right)$, which implies $Z_{i}\left(p^{*}\right)=\sum_{j=2}^{t+1} \lambda_{j}^{*} z_{i}\left(y^{j}\right) \geq 0$. On the other hand, since $i \in T^{-}$, we must have that $Z_{i}\left(p^{*}\right)=\alpha \leq 0$. This yields $Z_{i}\left(p^{*}\right)=\alpha=0$. Since $\alpha<0$, this possibility cannot occur.

In Possibility 2, the facet $\tau$ also lies in precisely one other subset of $A(T)$, say $A\left(\underline{T}^{0}, \underline{\Gamma}\left(\underline{T}^{1}\right)\right)$. Then, $\tau$ is a facet of a unique $t$-simplex $\sigma(\underline{a}, \underline{\pi})$ in this subset. The necessary adjustments to characterize $\sigma(\underline{a}, \underline{\pi})$ and $A\left(\underline{T}^{0}, \underline{\Gamma}\left(\underline{T}^{1}\right)\right)$ may be found in Table 2.2. The vertex $\underline{y}^{s}$ of $\sigma(\underline{a}, \underline{\pi})$ opposite the facet $\tau$ replaces $y^{s}$ of $\sigma(a, \pi)$ in system (2.1). Next a linear programming pivot step is performed with $\left[z\left(\frac{\left.y^{s}\right)}{1}\right]\right.$ by increasing $\lambda_{s}$ from zero. Thus, a line segment is followed. in $\sigma(\underline{a}, \underline{\pi})$. 


\begin{tabular}{|l|l|}
\hline Case & $\underline{T}^{0}, \underline{T}^{1}, \underline{\Gamma}\left(\underline{T}^{1}\right), \underline{a}, \underline{\pi}$ \\
\hline \multirow{4}{*}{$k>2$} & $\begin{array}{l}\underline{T}^{0}=T^{0}, \underline{T}^{1}=T^{1} \\
\underline{\Gamma}\left(\underline{T}^{1}\right)=\left(\Gamma_{1}, \ldots, \Gamma_{k-3}, \Gamma_{k-1}, \Gamma_{k-2}, \Gamma_{k}, \ldots, \Gamma_{t-1}\right) \\
\underline{a}=a, \underline{\pi}=\pi\end{array}$ \\
\hline & $\begin{array}{l}\text { if } \Gamma_{1}>0 \text { then } \\
k=2 \text { and }\end{array}$ \\
$\begin{array}{l}T^{0}=\{+h,-j\} \\
\end{array}$ & $\begin{array}{l}\underline{T}^{0}=\left\{\Gamma_{1},-j\right\}, \underline{T}^{1}=T \backslash \underline{T}^{0} \\
\underline{\Gamma}\left(\underline{T}^{1}\right)=\left(+h, \Gamma_{2}, \ldots, \Gamma_{t-1}\right) \\
\underline{a}=a, \underline{\pi}=\pi\end{array}$ \\
& $\begin{array}{l}\underline{T}^{0}=\left\{+h, \Gamma_{1}\right\}, \underline{T}^{1}=T \backslash \underline{T}^{0} \\
\underline{\Gamma}\left(\underline{T}^{1}\right)=\left(-j, \Gamma_{2}, \ldots, \Gamma_{t-1}\right) \\
\underline{a}=a, \underline{\pi}=\pi\end{array}$ \\
\hline
\end{tabular}

Table 2.2. Adaptations to be made when the facet $\tau$ opposite the vertex $y^{s}$ for some $s \in\{2, \ldots, t\}$ of $\sigma(a, \pi)$ in $A\left(T^{0}, \Gamma\left(T^{1}\right)\right)$ lies in $b d A\left(T^{0}, \Gamma\left(T^{1}\right)\right)$.

In the case of Possibility 3, the facet $\tau$ lies in $b d A(T)$ but not in $b d S^{n}$. More precisely, $\tau$ is a $T$-complete $(t-1)$-simplex in $A\left(T \backslash\left\{\Gamma_{t-1}\right\}\right)$. Then the element $\Gamma_{t-1}$ is removed from $T$ and from $T^{1}$ and the algorithm continues in $A(\underline{T})$ with $\underline{T}=T \backslash\left\{\Gamma_{t-1}\right\}$. The simplex $\tau$ is the $\underline{T}$-complete $(t-1)$-simplex. $\sigma(\underline{a}, \underline{\pi})$ in $A\left(\underline{T}^{0}, \underline{\Gamma}\left(\underline{T}^{1}\right)\right)$ described in Table 2.3. A linear programming pivot step is performed in system (2.1) with $\left(\begin{array}{c}e_{j} \\ 0\end{array}\right)$, where $j=\Gamma_{t-1}$ if $\Gamma_{t-1}>0$ and $j=-\Gamma_{t-1}$ if $\Gamma_{t-1}<0$, by decreasing $\mu_{j}$ from $\beta$ if $\Gamma_{t-1}>0$, and by increasing $\mu_{j}$ from $\alpha$ if $\Gamma_{t-1}<0$. Thus, $p_{j}$ is kept equal to $v_{j}$ and a line segment is followed in the simplex $\sigma(\underline{a}, \underline{\pi})$ of dimension one lower.

\begin{tabular}{|l|}
\hline$\underline{T}^{0}, \underline{T}^{1}, \underline{\Gamma}\left(\underline{T}^{1}\right), \underline{a}, \underline{\pi}$ \\
\hline$\underline{T}^{\mathrm{U}}=T^{0}, \underline{T}^{1}=T^{1} \backslash\left\{\Gamma_{t-1}\right\}$ \\
\hline$\underline{\Gamma}\left(\underline{T}^{1}\right)=\left(\Gamma_{1}, \ldots, \Gamma_{t-2}\right)$ \\
\hline$\underline{a}=a, \underline{\pi}=\left(\pi_{1}, \ldots, \pi_{t-1}\right)$ \\
\hline
\end{tabular}

Table 3.3. Adaptations to be made when the facet $\tau$ opposite vertex $y^{t+1}$ of $\sigma(a, \pi)$ in $A\left(T^{0}, \Gamma\left(T^{1}\right)\right)$ lies in $b d A(T)$.

In Situation 2, one of the variables $\mu_{h}, h \in T^{c}$, becomes equal to $\alpha$ or $\beta$. Let $j=h$ if $\mu_{h}=\alpha$, and let $j=-h$ if $\mu_{h}=\beta$. The $t$-simplex $\sigma(a, \pi)$ is the facet of exactly one $(t+1)$-simplex $\sigma(\underline{a}, \underline{\pi})$ in a subset $A\left(\underline{T}^{0}, \underline{\Gamma}\left(\underline{T}^{1}\right)\right)$ of $A(\underline{T})$ where $\underline{T}=T \cup\{j\}$, as described in Table 2.4. A linear programming pivot step is performed in system (2.1) with $\left[\begin{array}{c}z\left(\underline{y}^{t+2}\right) \\ 1\end{array}\right]$ by increasing $\lambda_{t+2}$ 
from zero, where $\underline{y}^{t+2}$ is the vertex of $\sigma(\underline{a}, \underline{\pi})$ opposite $\sigma(a, \pi)$. The algorithm increases $p_{h}$ from $v_{h}$ if $\mu_{h}=\alpha$ and decreases $p_{h}$ from $v_{h}$ if $\mu_{h}=\beta$. Thus a line segment in $\sigma(\underline{a}, \underline{\pi})$ is followed.

\begin{tabular}{|l|}
\hline$\underline{T}^{0}, \underline{T}^{1}, \underline{\Gamma}\left(\underline{T}^{1}\right), \underline{a}, \underline{\pi}$ \\
\hline$\underline{T}^{0}=T^{0}, \underline{T}^{1}=T^{1} \cup\{j\}$ \\
\hline$\underline{\Gamma}\left(\underline{T}^{1}\right)=\left(\Gamma_{1}, \ldots, \Gamma_{t-1}, j\right)$ \\
\hline$\underline{a}=a, \underline{\pi}=\left(\pi_{1}, \ldots, \pi_{t}, t+1\right)$ \\
\hline
\end{tabular}

Table 2.4. Adaptations to be made if $\mu_{h}$ has become equal to $\alpha$ or $\beta$; $j=-h$ if $\mu_{h}=\alpha$, and $j=h$ if $\mu_{h}=\beta$.

So, the algorithm terminates as soon as $\alpha=0$ or $\beta=0$. All components of $Z\left(p^{*}\right)$, where $p^{*}$ is the approximating solution, are nonpositive, in case $\beta=0$, or nonnegative, in case $\alpha=0$. The function $Z$ is an approximation of $z$, hence we have $z\left(p^{*}\right) \approx Z\left(p^{*}\right) \geq 0^{n+1}$, or $z\left(p^{*}\right) \approx Z\left(p^{*}\right) \leq 0^{n+1}$. This gives rise to the conclusion that $p^{*}$ is close to a solution, or at least to a point where all components of $z\left(p^{*}\right)$ are small in absolute value, since obviously $\left(p^{*}\right) \cdot z\left(p^{*}\right)=0$. How close all components of $z\left(p^{*}\right)$ are to zero depends on the grid size $m^{-1}$. If the accuracy of the approximating solution is not sufficient, the algorithm may be restarted in or close to $p^{*}$ with a smaller grid size in order to improve the accuracy of the approximating solution to be found in the next round. Within a finite number of restarts any a priori chosen accuracy of approximating solution is reached in this way.

\subsubsection{The steps of the algorithm}

The steps of the $n(n+1)$-ray algorithm to approximate a zero point of a continuous excess demand function $z$ on $S^{n}$, starting at an arbitrary $v \in$ int. $S^{n}$ and using the $Q V$-triangulation with grid size $m^{-1}$ as underlying simplicial subdivision of $S^{n}$, are as described below. This subsection ends with an example which illustrates the steps taken by the algorithm.

Step 0. Let $z_{k}(v)=\min _{h} z_{h}(v)$ and $z_{l}(v)=\max _{h} z_{h}(v)$. Set $y^{1}=v$, $T^{0}=\{+l,-k\}, T=T^{0}, T^{1}=\emptyset, t=1, \pi=(1), a=(0, \ldots, 0), w=2, \lambda_{1}=1$, $\alpha=z_{k}(v), \beta=z_{l}(v), \mu_{h}=z_{h}(v), h \in T^{c}$, and go to Step $\mathbf{1}$.

Step 1. Perform a linear programming pivot step with $\left[\begin{array}{c}z\left(y^{w}\right) \\ 1\end{array}\right]$ by increas- 
ing $\lambda_{w}$ from zero in the following system of linear equations:

$$
\sum_{i=1}^{t+1} \lambda_{i}\left[\begin{array}{c}
z\left(y^{i}\right) \\
1
\end{array}\right]-\sum_{h \in T^{e}} \mu_{h}\left[\begin{array}{c}
e_{h} \\
0
\end{array}\right]-\alpha\left[\begin{array}{c}
e\left(T^{-}\right) \\
0
\end{array}\right]-\beta\left[\begin{array}{c}
e\left(T^{+}\right) \\
0
\end{array}\right]=\left[\begin{array}{c}
0^{n+1} \\
1
\end{array}\right],
$$

where $\lambda_{i} \geq 0$ for all $i \in I^{t+1}, \alpha \leq 0, \beta \geq 0, \alpha \leq \mu_{h} \leq \beta$ for all $h \in T^{c}$. When $\alpha$ or $\beta$ becomes equal to zero, go to Step 3. Else, if $\mu_{h}$ becomes equal to $\alpha$ or $\beta$ for some $h \in T^{c}$, go to Step 3 .

Else $\lambda_{s}$ becomes equal to zero for some $s \in I^{t+1} \backslash\{w\}$, go to Step 2.

Step 2. If $2 \leq s \leq t, a_{\pi_{s-1}}=a_{\pi_{s}}, \pi_{s-1}=k-1$ and $\pi_{s}=k$ for a certain $k, 2 \leq k \leq t$, then we can find the necessary adjustments in Table 2.2, to obtain $\sigma(\underline{a}, \underline{\pi})$. Let $s^{\prime}$ be the index of the new vertex of $\sigma(\underline{a}, \underline{\pi})$, set $w=s^{\prime}$, $\sigma(a, \pi)=\sigma(\underline{a}, \underline{\pi})$, and return to Step 1 .

If $s=t+1, \pi_{t}=t$ and $a_{\pi_{t}}=0$, go to Step 4 .

Else replace $y^{s}$ according to Table 2.1 to obtain $\sigma(\underline{a}, \underline{\pi})$, set $w=s^{\prime}, \sigma(a, \pi)=$ $\sigma(\underline{a}, \underline{\pi})$, and return to Step 1 .

Step 3. If $\alpha=0$ or $\beta=0$, stop with solution $\left(\lambda^{*}, \mu^{*}, \alpha^{*}, \beta^{*}\right)$ and $p^{*}=$ $\sum_{i=1}^{t+1} \lambda_{i}^{*} y^{i}$ is an approximating zero point of $z$.

Else, for precisely one $h \in T^{c}$, it holds that $\mu_{h}=\alpha$ or $\mu_{h}=\beta$. If $\mu_{h}=\alpha$, then $-h$ is added to $T$, and if $\mu_{h}=\beta$, then $h$ is added to $T$. The relevant adaptations may be found in Table 2.4 to obtain $\sigma(\underline{a}, \underline{\pi})$, set $t=t+1, w=t+1$, $\sigma(a, \pi) \equiv \sigma(\underline{a}, \underline{\pi})$, and return to Step 1 .

Step 4. The set $T$ becomes $T \backslash\left\{\Gamma_{t-1}\right\}$. The other adjustments may be found in Table 2.3 to obtain $\sigma(\underline{a}, \underline{\pi})$, set $t=t-1, \sigma(a, \pi)=\sigma(\underline{a}, \underline{\pi})$, and perform a linear programming pivot step in (2.7) with $\left[\begin{array}{c}e_{j} \\ 0\end{array}\right]$ where $j=\left|\Gamma_{t-1}\right|$, by increasing the variable $\mu_{j}$ from $\alpha$ if $\Gamma_{t-1}<0$, and by decreasing the variable $\mu_{j}$ from $\beta$ if $\Gamma_{t-1}>0$. If either $\mu_{h}$ becomes equal to $\alpha$ or $\beta$ for a certain $h \in T^{c}$, or if $\alpha$ or $\beta$ becomes equal to zero, then return to Step 3. Else $\lambda_{s}$ becomes zero for some $s \in I^{t+1}$, and return to Step 2 .

Every round terminates at Step 3 with a solution $\left(\lambda^{*}, \mu^{*}, \alpha^{*}, \beta^{*}\right)$ satisfying $\alpha^{*}=0$ or $\beta^{*}=0$. The approximating solution $p^{*}$ therefore satisfies $Z_{j}\left(p^{*}\right) \leq$ $\beta=0$ for all $j \in I^{n+1}$, or $Z_{j}\left(p^{*}\right) \geq \alpha=0$ for all $j \in I^{n+1}$. Whenever this approximation of a zero point of $z$ is not satisfactory, i.e., $z\left(p^{*}\right)$ is not close 
enough to zero in all components, then the algorithm may be restarted with $v$ near $p^{*}$ and a larger $m$. In this manner, any a priori chosen accuracy of the approximating solution can be reached within a finite number of restarts.

The remainder of this subsection is devoted to a discussion of the algorithm for an example. Clearly, $p$ satisfies (2.6) if and only if $p \in A(T) \cap C(T)$ for some set $T$. The point $v$ in Figure 2.9 is identical to the $v$ of the Figures 2.1 to 2.8 . Since $v \in C(\{+3,-1\})$, at the starting point, the excess demand for commodity 3 is maximal, and the excess demand for commodity 1 is minimal. The excess demand for commodity 2 is neither maximal nor minimal. The point $p^{\prime}$ is the intersection point of all sets $C(T)$. At $p^{\prime}$ it holds that $z_{i}\left(p^{\prime}\right)=\max _{h \in I^{3}} z_{h}\left(p^{\prime}\right)$ for all $i \in I^{n+1}$. Simultaneously, $z_{i}\left(p^{\prime}\right)=\min _{h \in I^{3}} z_{h}\left(p^{\prime}\right)$ for all $i \in I^{n+1}$. Hence, by Lemma 2.4, $z\left(p^{\prime}\right)=0^{n+1}$,

In Figure 2.9, the grid size of the $Q V$-triangulation is $m^{-1}=\frac{1}{2}$. The vertices of simplices which are relevant for the discussion are denoted by $u^{1}, \ldots, u^{7}$. The points corresponding with basic solutions are denoted by $p^{1}, \ldots, p^{6}$, and the approximating solution reached by the algorithm is denoted by $p^{*}$. The piecewise linear path from $v$ to $p^{*}$, denoted by the heavily drawn path in Figure 2.9, approximately follows the path $P$ in Figure 2.7. On the piecewise linear path from $v$ to $p^{*}$, the algorithm generates a sequence of eight adjacent $T$-complete $t$-simplices in sets $A(T)$ for varying sets $T$ with $t=|T|-1: \sigma\left(v, u^{1}\right)$ in $A(\{+3,-1\}), \sigma\left(v, u^{1}, u^{2}\right)$ in $A(\{+3,-1\},(+2))$, and $\sigma\left(v, u^{3}, u^{2}\right)$ in $A(\{+2,-1\},(+3))$, then $\sigma\left(v, u^{3}\right)$ and $\sigma\left(u^{3}, u^{4}\right)$ in $A(\{+2,-1\})$, followed by $\sigma\left(u^{3}, u^{4}, u^{5}\right), \sigma\left(u^{3}, u^{6}, u^{5}\right)$, and $\sigma\left(u^{6}, u^{5}, u^{7}\right)$ in $A(\{+2,-1\},(-3))$.

The algorithm begins with Step 0 , and the initial settings relevant here, are $T=T^{0}:=\{+3,-1\}, T^{c}:=\{2\}, y^{1}:=v, y^{2}:=u^{1}, \lambda:=(1,0), \alpha:=$ $\min _{h \in I^{3}} Z_{h}(v)=Z_{1}(v), \beta:=\max _{h \in I^{3}} Z_{h}(v)=Z_{3}(v)$, and $\mu_{2}:=Z_{2}(v)$. The algorithm performs in Step 1, a linear programming pivot step in system (2.7) by increasing $\lambda_{2}$ from zero. A linear piece of points $p$ in $A(\{+3,-1\})$ is generated in $\sigma\left(v, u^{1}\right)$ satisfying $Z_{1}(p) \leq Z_{2}(p) \leq Z_{3}(p)$ and $p_{1} \leq v_{1}, p_{2}=v_{2}$, $p_{3} \geq v_{3}$. This linear piece in $\sigma\left(v, u^{1}\right)$ ends at $p^{1}$, which corresponds with the basic solution where $\mu_{2}=\beta$.

At the point $p^{1}$ in $\sigma\left(v, u^{1}\right)$, the second component of the piecewise linear approximation $Z$ of $z$ becomes maximal, i.e., $Z_{2}\left(p^{1}\right)=Z_{3}\left(p^{1}\right)$. The algorithm continues with Step 3, and makes the adjustments in Table 2.4: $T:=T \cup\{+2\}, T^{1}:=\{+2\}$, and $y^{3}:=u^{2}$. Note that the 1 -simplex $\sigma\left(v, u^{1}\right)$ is a $\{+2,+3,-1\}$-complete facet of the $\{+2,+3,-1\}$-complete 2 simplex $\sigma\left(v, u^{1}, u^{2}\right)$. The algorithm returns to Step 1 by making a linear programming pivot step in system (2.7) by raising $\lambda_{3}$ from zero. A linear 


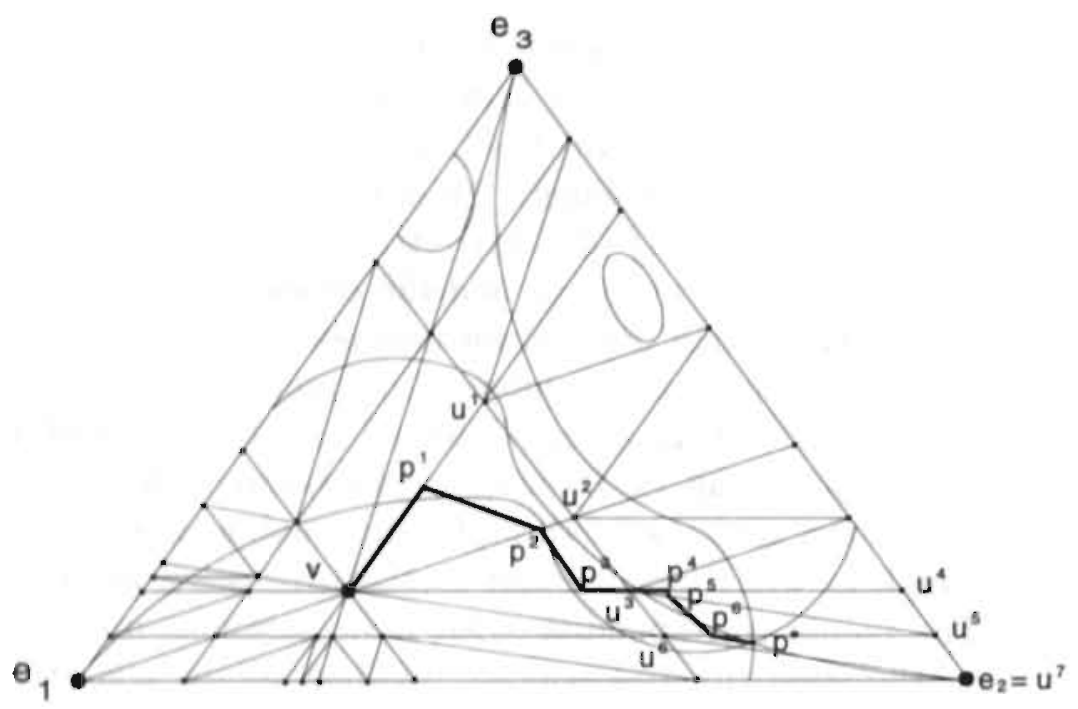

Figure 2.9: The piecewise linear path of points satisfying (2.2), followed by the algorithm from $v$ to the approximating solution $p^{*}$, is depicted as the sequence of seven connected heavy line segments.

piece of points $p$ in $A(\{+3,-1\},(+2))$ is generated in $\sigma\left(v, u^{1}, u^{2}\right)$ satisfying $Z_{1}(p) \leq Z_{2}(p)=Z_{3}(p)$ and $p_{1} \leq v_{1}, p_{2} \geq v_{2}, p_{3} \geq v_{3}$. This linear piece in $\sigma\left(v, u^{1}, u^{2}\right)$ ends at $p^{2}$ which corresponds with the basic solution where $\lambda_{2}=0$.

The point $p^{2}$ lies in the other $\{+2,+3,-1\}$-complete facet of $\sigma\left(v, u^{1}, u^{2}\right)$ with vertices $v$ and $u^{2}$. This facet lies in $\left.b d A(\{+3,-1\}),(+2)\right)$ and also in bd $A(\{+2,-1\},(+3))$. It forms a facet of precisely one simplex in the set $A(\{+2,-1\},(+3))$. The algorithm performs Step 2, and makes the adjustments in Table 2.2: $T^{0}:=\{+2,-1\}, T^{1}:=\{+3\}, y^{2}:=u^{3}, y^{3}:=$ $u^{2}$. The algorithm returns to Step 1, a linear programming pivot step is made in system (2.7) by raising $\lambda_{2}$ from zero. A linear piece of points $p$ in $A(\{+2,-1\},(+3))$ is generated in $\sigma\left(v, u^{3}, u^{2}\right)$ satisfying $Z_{1}(p) \leq Z_{2}(p)=$ $Z_{3}(p)$ and $p_{1} \leq v_{1}, p_{2} \geq v_{2}, p_{3} \geq v_{3}$. This linear piece in $\sigma\left(v, u^{3}, u^{2}\right)$ ends at $p^{3}$ which corresponds with the basic solution where $\lambda_{3}=0$.

The point $p^{3}$ lies in the $\{+2,+3,-1\}$-complete facet of $\sigma\left(v, u^{3}, u^{2}\right)$ with 
vertices $v$ and $u^{3}$. This facet lies in $b d A(\{+2,+3,-1\})$, but not in $b d S^{n}$. This boundary piece is formed by the set $A(\{+2,-1\})$ and the facet is the $\{+2,-1\}$-complete 1 -simplex $\sigma\left(v, u^{3}\right)$ in $A(\{+2,-1\})$. The algorithm performs Step 2 and continues with Step 4 by setting $T:=T \backslash\{+3\}$ and by making the adjustments in Table 2.3: $y^{1}:=v, y^{2}:=u^{3}$, while $\beta:=Z_{2}\left(p^{3}\right)$ and $\mu_{3}:=Z_{3}\left(p^{3}\right)$. The algorithm returns to Step 1, a linear programming pivot step in system (2.7) is made by decreasing $\mu_{3}$ from $\beta$. A linear piece of points $p$ in $A(\{+2,-1\})$ is generated in $\sigma\left(v, u^{3}\right)$ satisfying $Z_{1}(p) \leq Z_{3}(p) \leq Z_{2}(p)$ and $p_{1} \leq v_{1}, p_{2} \geq v_{2}, p_{3}=v_{3}$. This linear piece in $\sigma\left(v, u^{3}\right)$ ends at $y^{2}=u^{3}$ which corresponds with the basic solution where $\lambda_{1}=0$.

The set $\left\{u^{3}\right\}$ is a $\{+2,-1\}$-complete facet of $\sigma\left(y, u^{3}\right)$, and a facet of precisely one other simplex in $A(\{+2,-1\})$. The algorithm performs Step 2 and the adjustments in Table 2.1: $y^{1}:=u^{3}, y^{2}:=u^{4}$. The algorithm returns to Step 1, a linear programming pivot step is made in system (2.7) by increasing $\lambda_{2}$ from zero. Thus, a linear piece of points $p$ in $A(\{+2,-1\})$ is generated in $\sigma\left(u^{3}, u^{4}\right)$ satisfying $Z_{1}(p) \leq Z_{3}(p) \leq Z_{2}(p)$ and $p_{1} \leq v_{1}$, $p_{2} \geq v_{2}, p_{3}=v_{3}$. This linear piece in $\sigma\left(u^{3}, u^{4}\right)$ ends at $p^{4} \in$ int $\sigma\left(u^{3}, u^{4}\right)$ which corresponds with the basic solution where $\mu_{3}=\alpha$.

At $p^{4}$ it holds that $Z_{1}\left(p^{4}\right)=Z_{3}\left(p^{4}\right)=\min _{h \in I^{3}} Z_{h}\left(p^{4}\right)$. The point $p^{4}$ lies in the $\{+2,-1,-3\}$-complete facet $\sigma\left(u^{3}, u^{4}\right)$ of $\sigma\left(u^{3}, u^{4}, u^{5}\right)$. The algorithm performs Step 3, and makes the adjustments in Table 2.4: $T:=T \cup\{-3\}$, $T^{1}:=\{-3\}, y^{3}:=u^{5}$. The algorithm returns to Step 1, a linear programming pivot step is made by raising $\lambda_{3}$ from zero. A linear piece of points $p$ in $A(\{+2,-1\},(-3))$ is generated in $\sigma\left(u^{3}, u^{4}, u^{5}\right)$ satisfying $Z_{1}(p)=Z_{3}(p) \leq$ $Z_{2}(p)$ and $p_{1} \leq v_{1}, p_{2} \geq v_{2}, p_{3} \leq v_{3}$. This linear piece in $\sigma\left(u^{3}, u^{4}, u^{5}\right)$, ends at $p^{5}$ which corresponds with the basic solution where $\lambda_{2}=0$.

The point $p^{5}$ lies in the other $\{+2,-1,-3\}$-complete facet of $\sigma\left(u^{3}, u^{4}, u^{5}\right)$, and this facet is a $\{+2,-1,-3\}$-complete facet of precisely one other simplex in $A(\{+2,-1\},(-3))$. The algorithm performs Step 2, and makes the adjustments in Table 2.1: $y^{2}:=u^{6}, y^{3}:=u^{5}$. The algorithm returns to $\mathrm{Step}$ 1 , and performs a linear programming pivot step in (2.7) by increasing $\lambda_{2}$ from zero. Thus, a linear piece of points $p$ in $A(\{+2,-1\},(-3))$ is generated in $\sigma\left(u^{3}, u^{6}, u^{5}\right)$ satisfying $Z_{1}(p)=Z_{3}(p) \leq Z_{2}(p)$ and $p_{1} \leq v_{1}, p_{2} \geq v_{2}$, $p_{3} \leq v_{3}$. This linear piece in $\sigma\left(u^{3}, u^{6}, u^{5}\right)$ ends at $p^{6}$ corresponding with the basic solution where $\lambda_{1}=0$.

The point $p^{6}$ lies in the other $\{+2,-1,-3\}$-complete facet of $\sigma\left(u^{3}, u^{6}, u^{5}\right)$, and this facet is a $\{+2,-1,-3\}$-complete facet of precisely one other simplex in $A(\{+2,-1\},(-3))$. The algorithm performs Step 2, and makes the 
adjustments in Table 2.1: $y^{1}:=u^{6}, y^{2}:=u^{5}, y^{3}:=u^{7}$. The algorithm returns to Step 1, and performs a linear programming pivot step in system (2.7) by increasing $\lambda_{3}$ from zero. A linear piece of points $p$ in $\sigma\left(u^{6}, u^{5}, u^{7}\right)$ is generated satisfying $Z_{1}(p)=Z_{3}(p) \leq Z_{2}(p)$ and $p_{1} \leq v_{1}, p_{2} \geq v_{2}, p_{3} \leq v_{3}$, which ends at $p^{*}$. The algorithm terminates with the solution $\left(\lambda^{*}, \mu^{*}, \alpha^{*}, \beta^{*}\right)$, and $p^{*}=\sum_{i=1}^{3} \lambda_{i}^{*} y^{i} \in$ int $\sigma\left(u^{6}, u^{5}, u^{7}\right)$ is an approximation of the equilibrium price vector $p^{\prime}$.

If $z\left(p^{*}\right)$, the excess demand function evaluated at $p^{*}$, is not close enough to zero in all components, then the algorithm is restarted at $p^{*}$, with a finer simplicial subdivision of the unit simplex, i.e., a larger $m$. Such restarts with a finer simplicial subdivision of the unit simplex, are made by the algorithm until a round terminates with an approximation that satisfies the a-priori chosen accuracy level.

\subsection{An intersection theorem}

The $n(n+1)$-ray algorithm leads directly to an intersection theorem of closed subsets covering $S^{n}$. This theorem can be considered as a hybrid of the well-known lemma of Knaster, Kuratowski and Mazurkiewicz (KKM) and an intersection result on the unit simplex of Freidenfelds. Both intersection theorems follow namely immediately as corollaries. In the next theorem $S^{n}$ is covered by $n(n+1)$ closed subsets. Some of these subsets might be empty. Each subset of the collection is indexed by a pair $(+i,-j)$ with $i, j \in I^{n+1}$.

Theorem 2.12 Let $\left\{B(+i,-j) \mid i, j \in I^{n+1}, i \neq j\right\}$ be a family of closed sets covering $S^{n}$ such that for every $x \in b d S^{n}$ it holds that $x \in B(+i,-j)$ for some $i \in I^{n+1}$ and for some $j \in I^{n+1}$ satisfying $x_{j}>0$, or for every $i \in I^{n+1}$ satisfying $x_{i}=0, x \in B(+i,-j)$ for some $j \in I^{n+1}$. Then there exist an $x^{*} \in S^{n}$ and a nonempty set $T^{*} \subset I^{n+1}$ satisfying that for every $i \in T^{*}$ there exist $j \in T^{*}$ and $k \in T^{*}$ such that $x^{*} \in B(+i,-j)$ and $x^{*} \in B(+k,-i)$.

Proof. For $x \in S^{n}$, let $z: S^{n} \rightarrow \Re^{n+1}$ be defined by $z(x)=e(i)-e(j)$ for some $i, j \in I^{n+1}$ satisfying $x \in B(+i,-j)$ where $x_{j}>0$ and if no such $j \in I^{n+1}$ exists then $i$ satisfies $x_{i}=0$. Clearly, $\sum_{l=1}^{n+1} z_{l}(x)=0$ for all $x \in S^{n}$. Given some fixed starting point $v \in$ int $S^{n}$ let the $n(n+1)$-ray algorithm be applied to $z$ for a sequence of decreasing grid sizes $m_{1}^{-1}, m_{2}^{-1}, m_{3}^{-1}, \ldots$, converging to zero.

Suppose the algorithm terminates at $x^{*}$ in $b d S^{n}$ satisfying $x_{j}^{*}=0$ for every 
$j$ for which $x^{*}$ lies in $B(+i,-j)$ for some $i \in I^{n+1}$. Then for every $i \in I^{n+1}$ satisfying $x_{i}^{*}=0$ it holds that $x^{*} \in B\left(+i,-j_{i}\right)$ for some $j_{i} \in I^{n+1}$ with $x_{j_{i}}^{*}=0$. Clearly, $x^{*}$ satisfies the statement in the theorem with $T^{*}$ equal to the set $\left\{j_{i} \in I^{n+1} \mid x^{*} \in B\left(+i,-j_{i}\right)\right.$ and $\left.x_{i}^{*}=0\right\}$.

Otherwise in round $k, k=1,2, \ldots$, the algorithm terminates with a, say, $t_{k}$-dimensional simplex $\sigma^{k}$ with vertices $y^{k, 1}, \ldots, y^{k, t_{k}+1}$ such that the system of linear equations

$$
\sum_{h=1}^{t_{k}+1} \lambda_{h}^{k}\left(e_{i_{h}^{k}}-e_{j k}\right)=0,
$$

has a nonnegative solution $\lambda_{1}^{k}, \ldots, \lambda_{t_{k}+1}^{k}$ summing up to one and where $i_{h}^{k}, j_{h}^{k} \in$ $I^{n+1}$ are such that the vertex $y^{k, h}$ of $\sigma^{k}$ lies in $B\left(+i_{h}^{k},-j_{h}^{k}\right)$, for $h=1, \ldots, t_{k}+1$. Notice that due to the fact that $\sum_{l=1}^{t_{k}+1} z_{l}\left(y^{k, h}\right)=0$ for every $h=1, \ldots, t_{k}+1$, the algorithm can only terminate at Step 3 with both $\alpha$ and $\beta$ equal to zero. Let $x^{k}=\sum_{h=1}^{t_{k}+1} \lambda_{h}^{k} y^{k, h}$ be the approximating solution found in round $k, k=$ $1,2, \ldots$. When $k$ goes to infinity then there exists a subsequence $k_{1}, k_{2}, \ldots$, such that $\lim _{\nu \rightarrow \infty} x_{k_{\nu}}=x^{*}$ for some $x^{*} \in S^{n}, t_{k_{v}}=t^{*}$ for all $\nu, \lim _{\nu \rightarrow \infty} \lambda_{h}^{k_{\nu}}=$ $\lambda_{h}^{*} \geq 0$ for all $h=1, \ldots, t^{*}+1$, satisfying $\sum_{h=1}^{t^{*}+1} \lambda_{h}^{*}=1$, and $i_{h}^{k_{\nu}}=i_{h}$ and $j_{h}^{k_{\nu}}=j_{h}$ for some $i_{h}$ and $j_{h}, h=1, \ldots, t^{*}+1$. Since the grid size $m_{k_{\nu}}^{-1}$ converges to zero when $k_{\nu}$ goes to infinity, we also have that $y^{k_{\nu}, h}$ converges to $x^{*}$ for every $h=1, \ldots, t^{*}+1$. Since for every $h$ we have that $y^{k_{\nu}, h} \in B\left(+i_{h}^{k_{\nu}},-j_{h}^{k_{\nu}}\right)$ and every $B(+i,-j)$ is closed, we obtain that $x^{*} \in B\left(+i_{h},-j_{h}\right)$ for $h=$ $1, \ldots, t^{*}+1$. Furthermore it follows from (2.8), when $k=k_{\nu}$ and $\nu$ goes to infinity

$$
\sum_{h=1}^{t^{*}+1} \lambda_{h}^{*}\left(e_{i_{h}}-e_{j_{h}}\right)=0 .
$$

Now, let $T^{1}=\left\{i_{h} \mid \lambda_{h}^{*}>0, h \in I^{t^{*}+1}\right\}$ and let $T^{2}=\left\{j_{h} \mid \lambda_{h}^{*}>0, h \in I^{t^{*}+1}\right\}$. We will show that $T^{1}=T^{2}$. Take some $i \in T^{1}$ and suppose $i \notin T^{2}$. Then the $i$-th equation of (2.9) yields

$$
0=\sum_{h=1}^{t^{*}+1} \lambda_{h}^{*}\left(e_{i_{h}}\right)_{i} \geq \lambda_{k}^{*}>0
$$

where $k$ is such that $i_{k}=i$, yielding a contradiction. Similarly, take some $j \in T^{2}$ and assume $j \notin T^{1}$. Then the $j$-th equation of (2.9) yields

$$
0=-\sum_{h=1}^{t^{*}+1} \lambda_{h}^{*}\left(e_{j_{h}}\right)_{j} \leq-\lambda_{k}^{*}<0
$$




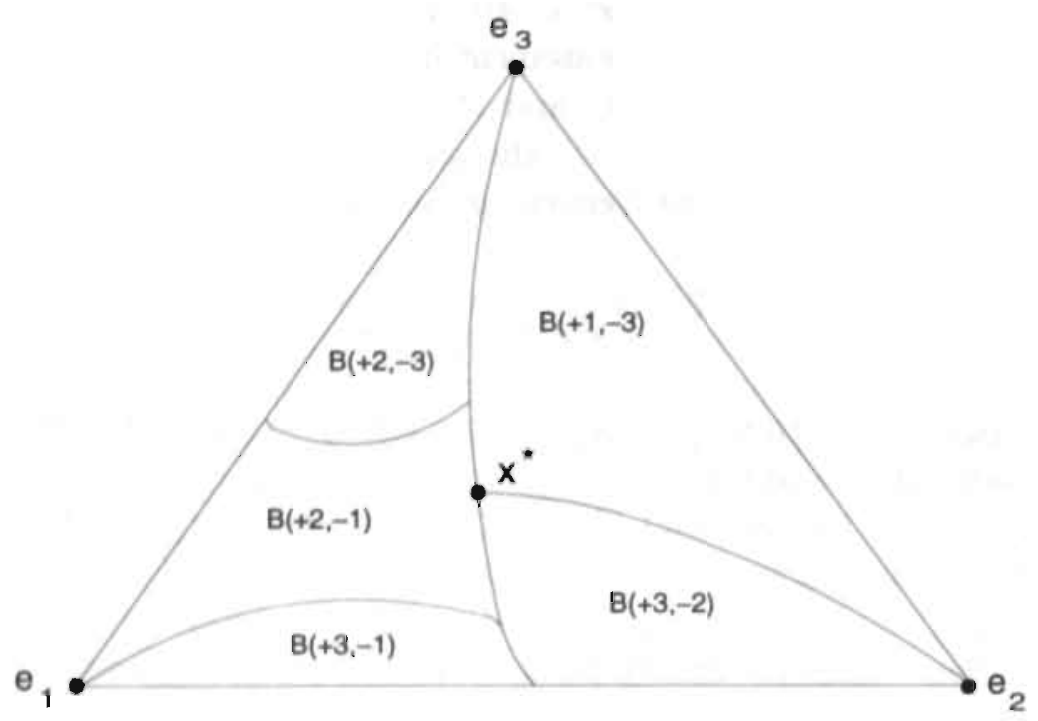

Figure 2.10: Illustration of Theorem 2.12, the intersection point is $x^{*}, T^{* *}=$ $\{1,2,3\}$.

where $k$ is such that $j_{k}=j$, yielding also a contradiction.

Hence, $T^{*}=T^{1}=T^{2}$ satisfies the statement of the theorem. Notice that $T^{*} \neq \emptyset$ since $\sum_{h=1}^{t^{*}+1} \lambda_{h}^{*}=1$.

The intersection theorem is illustrated for $n=2$ in Figures 2.10 and 2.11. In Figure 2.10, the set $T^{*}$ equals $\{1,2,3\}$ and $x^{*}$ lies in the intersection of $B(+1,-3), B(+2,-1)$ and $B(+3,-1)$. In Figure 2.11, the set $T^{*}$ equals $\{1,3\}$ and the set $B(+1,-3) \cap B(+3,-1)$ consists of the point $e_{2}$. From Theorem 2.12 both the KKM-lemma and Freidenfelds' intersection result on the unit simplex follow as corollaries.

Corollary 2.13 (KKM-lemma) Let $C_{1}, \ldots, C_{n+1}$ be $n+1$ closed subsets covering $S^{n}$ such that for every $x \in S^{n}$ there exists a $j \in I^{n+1}$ satisfying $x_{j}>0$ and $x \in C_{j}$. Then $\cap_{j=1}^{n+1} C_{j} \neq \emptyset$.

Proof. For $j=1, \ldots, n$, define $B(+(j+1),-j)=C_{j}$, where $j+1=1$ for $j=n+1$. Moreover $B(+i,-j)=\emptyset$ whenever $i \neq j+1$. Then these 
sets satisfy the conditions of Theorem 2.12. Hence, there exists $x^{*} \in S^{n}$ and a nonempty set $T^{*} \subseteq I^{\mathrm{n}+1}$ such that $j \in T^{*}$ implies $(j+1) \in T^{*}$ and $x^{*} \in B(+(j+1),-j)$. Hence $T^{*}=I^{n+1}$ and so $x^{*} \in C_{j}$ for every $j \in I^{n+1}$.

Similarly, the next intersection theorem follows.

Corollary 2.14 (Freidenfelds' intersection theorem on $S^{n}$ ) Let $C_{1}, \ldots, C_{n+1}$ be $n+1$ closed subsets covering $S^{n}$ such that $x \in C_{j}$ whenever $x_{j}=0$. Then $\cap_{j=1}^{n+1} C_{j} \neq \emptyset$.

Proof. For $j=1, \ldots, n+1$, define $B(+j,-(j+1))=C_{j}$ where $j+1=1$ when $j=n+1$. For $i \neq j+1$, let $B(+j,-i)=\emptyset$. We will first show that these sets satisfy the conditions of Theorem 2.12. Let $x \in b d\left(S^{n}\right)$. Then there exists a $j \in I^{n+1}$ such that $x_{j}=0$ and $x_{j+1}>0$. Since $x_{j}=0$ we have that $x \in C_{j}$. Hence, $x \in B(+j,-(j+1))$ whereas $x_{j+1}>0$. According to Theorem 2.12 there exist an $x^{*} \in S^{n}$ and a nonempty set $T^{*}$ satisfying $j \in T^{*}$ implies both $j+1 \in T^{*}$ and $x^{*} \in B(+j,-(j+1))$. Hence, $T^{*}=I^{n+1}$ and so $x^{*} \in C_{j}$ for all $j \in I^{n+1}$.

Proposition 2.15 For every excess demand function $z: S^{n} \rightarrow \Re^{n+1}$ there exists an economic equilibrium, i.e., there exists $p^{*} \in S^{n}$ satisfying $z\left(p^{*}\right)=$ $0^{n+1}$.

Proof. Let $z: S^{n} \rightarrow \Re^{n+1}$ be an excess demand function. For $i$ and $j \in I^{n+1}$, let us define

$$
B(+i,-j)=\left\{x \in S^{n} \mid z_{i}(x)=\max _{h \in I^{n+1}} z_{h}(x) \text { and } z_{j}(x)=\min _{h \in I^{n+1}} z_{h}(x)\right\} .
$$

Since $z$ is continuous, $p \cdot z(p)=0$, and $z_{h}(x) \geq 0$ whenever $x_{h}=0$, these sets satisfy the conditions of Theorem 2.12. Hence there exists an $x^{*} \in S^{n}$ and a nonempty $T^{*} \subseteq I^{n+1}$, such that $i \in T^{*}$ implies there exist a $j$ with $x^{*} \in B(+i,-j)$ and a $k$ with $x^{*} \in B(+k,-i)$. Consequently, $z_{i}\left(x^{*}\right)=\max _{h \in I^{n+1}} z_{h}\left(x^{*}\right)$ and $z_{i}\left(x^{*}\right)=\min _{h \in I^{n+1}} z_{h}\left(x^{*}\right)$, hence $z\left(x^{*}\right)=0^{n+1}$ by Lemma 2.4 . 


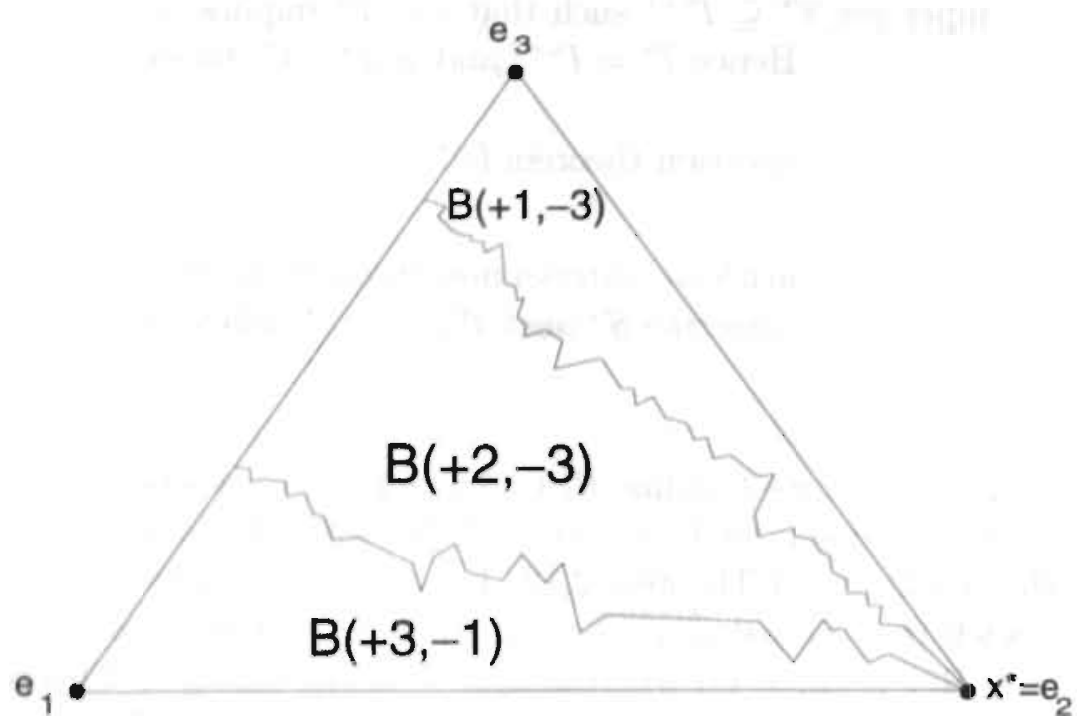

Figure 2.11: Illustration of Theorem $2.12, x^{*}$ is the intersection point, $T^{*}=$ $\{1,3\}$.

\subsection{References}

Arrow, K.J., \& L. Hurwicz, 1958, On the stability of the competitive equilibrium I, Econometrica 26, 522-552.

Arrow, K.J., H.D. Block, \& L. Hurwicz, 1959, On the stability of the competitive equilibrium II, Econometrica 27, 82-109.

Debreu, G., 1974, Excess demand functions, Journal of Mathematical Economics 1, 15-23.

Doup, T.M., 1988, "Simplicial Algorithms on The Simplotope", Springer, Berlin.

Doup, T.M., G. van der Laan, \& A.J.J. Talman, 1987, The $\left(2^{n+1}-2\right)$ ray algorithm: A new simplicial algorithm to compute economic equilibria, 
Mathematical Programming 39, 241-252.

Doup, T.M., \& A.J.J. Talman, 1987a, A new simplicial variable dimension algorithm to find equilibria on the product space of unit simplices, Mathematical Programming 37, 319-355.

Doup, T.M., \& A.J.J. Talman, 1987b, The 2-ray algorithm for solving equilibrium problems on the unit simplex, Methods of Operations Research $12,485-521$.

Elzen, A.H. van den, 1993, "Adjustment Processes for Exchange Economies and Noncooperative Games", Springer, Berlin.

Freidenfelds, J., 1974, An intersection theorem and applications, Mathematical Programming 7, 199-211.

Herings, P.J.J., 1994, A globally and universally stable price adjustment process, CentER Discussion. Paper 9452.

Herings, P.J.J., 1995, "Static and Dynamic Aspects of General Disequilibrium Theory", Ph.D. Thesis, CentER Dissertation 1, Tilburg University, Tilburg.

Joosten, R.A.M.G., \& A.J.J. Talman, 1993, A simplicial variable dimension restart algorithm to find economic equilibria on the unit simplex using $n(n+1)$ rays, Report M 93-01, Department of Mathematics, University of Limburg, Maastricht.

Joosten, R.A.M.G., \& A.J.J. Talman, 1995, A globally convergent price adjustment process for exchange economies, forthcoming in Journal of Mathematical Economics.

Kamiya, K., 1990, A globally stable price adjustment process, Econometrica $58,1481-1485$.

Keenan, D., 1981, Further remarks on the Global Newton method, Journal of Mathematical Economics 8, 159-165. 
Knaster, B., C. Kuratowski, \& S. Mazurkiewicz, 1929, Ein Beweis des Fixpunktsatzes für n-dimensionale Simplexe, Fundamenta Mathematicae 14, 132-137.

Kuhn, H.W., 1968, Simplicial approximation of fixed points, Proceedings National Academy of Sciences USA 61, 1238-1242.

Kuhn, H.W., 1969, Approximate search for fixed points, Computing Methods in Optimization Problems 2, 199-211.

Laan, G. van der, \& A.J.J. Talman, 1979, A restart algorithm for computing fixed points without an extra dimension, Mathematical Programming 17, 74-84.

Laan, G. van der, \& A.J.J. Talman, 1980, An improvement of fixed point algorithms by using a good triangulation, Mathematical Programming 18, 274-285.

Laan, G. van der, \& A.J.J. Talman, 1987a, Adjustment processes for finding economic equilibria, in "The Computation and Modelling of Economic Equilibria" (A.J.J. Talman and G. van der Laan, Eds.), North-Holland, Amsterdam.

Laan, G. van der, \& A.J.J. Talman, 1987b, A convergent price adjust-ment process, Economics Letters 23, 119-123.

Laan, G. van der, A.J.J. Talman, \& L. Van der Heyden, 1987, Simplicial variable dimension algorithms for solving the nonlinear complementarity problem on a product space of unit simplices using a. general labelling, Mathematics of Operations Research 12, 377-397.

Laan, G. van der, \& A.J.J. Talman, 1993, Intersection theorems on the simplotope, CentER Discussion Paper 9370, Tilburg University, Tilburg.

Mantel, R., 1974, On the characterization of aggregate excess demand, Journal of Economic Theory 7, 348-353.

Saari, D.G., \& C. Simon, 1978, Effective price mechanisms, Econometrica 
46, 1097-1125.

Samuelson, P., 1947, "Foundations of Economic Analysis", Harvard University Press, Cambridge.

Scarf, H., 1960, Some examples of global instability of competitive equilibrium, International Economic Review 1, 157-172.

Scarf, H., 1967, The approximation of fixed points of a continuous mapping, SIAM Journal of Applied Mathematics 15, 1328-1343.

Scarf, H., 1973 (with the collaboration of T. Hansen), "The Computation of Economic Equilibria", Yale University Press, New Haven.

Smale, S., 1976, A convergent process of price adjustment and global Newton methodes, Journal of Mathematical Economics 3, 107-120.

Sonnenschein, H., 1972, Market excess demand functions, Econometrica 40, 549-563.

Sonnenschein, H., 1973, Do Walras' identity and continuity characterize community excess demand functions?, Journal of Economic Theory 6, 345354.

Talman, A..J.J., 1994, Intersection theorems on the unit simplex and the simplotope, in: "Imperfections and Behavior in Economic Organization", R.P. Gilles and P.H.M. Ruys (eds.), Kluwer, Dordrecht, pp. 257-278.

Uzawa, H., 1961, The stability of dynamic processes, Econometrica 29. 617-631.

Walras, L., 1874, "Élements d'Économie Politique Pure", Corbaz, Lausanne.

Yang, Z., 1996, "Simplicial Fixed Point Algorithms and Applications", Ph.D. Thesis, CentER Dissertation 9, Tilburg University, Tilburg. 



\section{Chapter 3}

\section{Evolution of populations and strategies}

\subsection{Introduction}

In this chapter, we develop a framework for a more unified modelling of 'evolutionary" processes in the social sciences. We present two different evolutionary models, but apply an essentially similar method of analysis. In the first model, we call evolutionary dynamics sign-compatible if for every subgroup with positive population share, the change in population share corresponds in sign with its relative fitness, i.e., the difference between the subgroup's fitness and the average fitness. In the second model, the evolutionary dynamics are called sign-compatible if additionally for each subgroup the change in the weight on each action corresponds in sign with its relative payoff, i.e., the difference between this action's payoff and the average payoff on the subgroup's action. Sign-compatible dynamics form a large class of dynamics to which for instance the replicator dynamics (Taylor \& Jonker [1978]), as well as the class of aggregate monotonic dynamics (Samuelson \& Zhang [1992]) belong. An even larger class is formed by the class of the weakly sign-compatible dynamics. Evolutionary dynamics are called weakly sign-compatible if at every state at least one subgroup with increasing population share has above average fitness. In the second model, it must additionally hold for each subgroup that the weight on at least one action with increasing weight has above average payoff.

In evolutionary game theory the main equilibrium concepts are the Nash 
equilibrium (Nash [1951]) and the evolutionarily stable strategy (Maynard Smith \& Price [1973]). We forward the saturated equilibrium (Hofbauer \& Sigmund [1988]) as a generalization of the Nash equilibrium. We generalize the evolutionarily stable strategy into two separate directions: a dynamic and a static one. The dynamic generalization is called the evolutionarily stable equilibrium, the static version is called the generalized evolutionarily stable state. These equilibrium concepts will be applied to both evolutionary models.

A saturated equilibrium implies 'extinction of the less fit': each subgroup with below-maximal fitness has population share equal to zero. In the case that the concept of a saturated equilibrium is applied to the model where all subgroups learn, it must additionally hold that no subgroup places positive weight on any action with below-maximal payoff. We prove existence of at least one saturated equilibrium, and show that each saturated equilibrium is a fixed point for weakly sign-compatible dynamics. Furthermore, sign-compatible dynamics converge only to a saturated equilibrium from the interior of the state space, and all stable fixed points of sign-compatible dynamics are saturated. These results are proven for both models.

An evolutionarily stable equilibrium satisfies the property that for given weakly sign-compatible dynamics, any trajectory sufficiently nearby converges monotonically to it. Hence, each evolutionarily stable equilibrium is an asymptotically stable fixed point of the dynamics. Asymptotic stability of a fixed point means that if a (sufficiently) small perturbation of the dynamical system takes the system out of the fixed point, then the system will return to this fixed point again. Evolutionary stability in the dynamic sense additionally implies that the manner in which the system returns to the fixed point is 'well-behaved'. To determine whether a point is an evolutionarily stable equilibrium, it is necessary to know the dynamics nearby.

To establish whether a saturated equilibrium is a generalized evolutionarily stable state, only information about the payoff structure is needed. 'The conditions of a generalized evolutionarily stable state do not imply dynamic stability of the state for arbitrary (weakly) sign-compatible dynamics. However, for the replicator dynamics the generalized evolutionarily stable state is asymptotically stable.

Useful refinements of the saturated equilibrium concepts are the strict saturated equilibrium for the first model and the monopolistic and strict saturated equilibrium for the second model. At a strict saturated equilibrium precisely one subgroup has highest fitness. At a monopolistic and strict 
equilibrium additionally each subgroup possesses precisely one action with highest payoff. For these (strong) refinements the static and the dynamic variants of evolutionary stability do concur. These results are proven for both models.

Evolutionary dynamics were originally exclusively used as population dynamics, i.e., to model the evolution of the composition of a population over its subgroups, or to model the evolution of an ecological system with several species of organisms. However, at least since Zeeman [1981] evolutionary dynamics have been interpreted alternatively as learning or imitation dynamics in game theory. Developments may be roughly described as follows. At first, replicator dynamics were taken as a starting point of analysis, i.e., the dynamics were assumed to be the product of some unmodelled learning or imitation process. Lacking a compelling rationale for the replicator dynamics outside mathematical biology, larger classes of dynamics were then examined, e.g., Friedman [1991], Samuelson \& Zhang [1992], Swinkels [1993]. Experiments on learning meanwhile, seemed to justify the assumption that adaptive stategic adjustment behavior leads to evolutionary dynamics, cf. e.g., Crawford [1991], Miller \& Andreoni [1991]. Recently, several contributions have given explicit theoretical foundations for evolutionary dynamics as imitation or learning dynamics. Schlag [1994], Björnerstedt \& Weibull [1993], and Weibull [1995] derive evolutionary dynamics from imitation. Börgers \& Sarin [1995] derive the replicator dynamics in a learning-by-reinforcement model.

Best-response dynamics (Gilboa \& Matsui [1991], Matsui [1992]), which are closely related to continuous fictitious play dynamics (Rosenmüller [1971]), are often motivated by strategical inertia: every period each player receives an opportunity to adjust his strategy with a small probability. Assuming that individuals are rational, that they can calculate the best response in the current strategic environment, and that they can switch to this best response, the best-response dynamics appear on aggregate level as a strategy adjustment process. We refer to Gilboa \& Matsui [1991] and Matsui [1992] for more elaborate motivations and interpretations, and to Hofbauer [1995] and Oechssler [1995] for variations.

Several interpretations are possible for the two models in this chapter. In a purist interpretation, the first model describes the evolution of a population consisting of several interacting subgroups with fixed inheritable strategies. Alternatively, this model can be interpreted as describing a process of learning-by-imitation as in Zeeman [1981], or adaptive learning with strategic 
inertia as in Gilboa \& Matsui [1991] and Matsui [1992]. The purist interpretation of the second model is that it combines both population dynamics and learning dynamics for each subgroup in the population. An alternative interpretation, which may be attractive in mathematical biology, is that the model describes the coevolution of several species with distinguishable subgroups. The 'intermediate' model, namely a model with several subgroups which alter their strategies in an adaptive manner in a certain strategic environment, is covered by Friedman [1991].

The social sciences use variants of evolutionary dynamics to motivate several adaptive processes such as learning processes, selection processes, market share dynamics, etc. (e.g., Silverberg [1988], Dow [1986]). In the settings which are of interest in the social sciences, externalities may very well occur (e.g., Schelling [1978], Arthur [1990], Metcalfe [1994]). Externalities go by different names, such as self-reinforcing mechanisms, complementarities, state dependent feedbacks, increasing or decreasing returns, and may lead to non-linearities of the fitness functions. Our approach is general enough to deal with externalities.

Multiplicity of evolutionarily stable equilibria or generalized evolutionarily stable states may complicate predicting the long run evolution of the population as well. It would be quite helpful if conditions could be derived guaranteeing uniqueness of a saturated equilibrium, and simultaneously providing information on its dynamic stability. Several results on special cases exist, see e.g., Robinson [1951], Hofbauer \& Sigmund [1988], Nachbar. [1990], Monderer \& Sela [1993], Monderer \& Shapley [1993a,b], Hofbauer [1995], Oechssler [1995]. However, these results require at least considerable adaptation for nonlinear fitness functions and (weakly) sign-compatible dynamics.

The evolutionary process in our first model is driven by the so-called relative fitness function. A relative fitness function attributes for every state to each subgroup the difference between its fitness and the average fitness of the population. Each relative fitness function satisfies continuity and complementarity. The results of Sonnenschein [1972, 1973], Mantel [1974], and Debreu [1974] imply that any function satisfying continuity and complementarity is an excess demand function for a pure exchange economy with normalized prices. So, a relative fitness function may be regarded as an excess demand function for a pure exchange economy with prices normalized to the unit simplex. Further analogies to concepts in general equilibrium theory follow from there. We 'translate' several results from general equilibrium theory to derive further conditions for uniqueness of a saturated equilibrium 
for the 'population dynamics' model.

It is difficult in general to find (compute) a saturated equilibrium. For linear fitness functions, Jansen [1987] has formulated a linear programming algorithm. However, as far as we know Jansen's algorithm is only applicable to special cases of the first model in this chapter. The procedure of Van den Elzen \& Talman [1991] may be used for asymmetric evolutionary bi-matrix games. For nonlinear (relative) fitness functions more powerful methods are required e.g., Doup et al. [1987]. This algorithm finds a saturated equilibrium for both models presented in this chapter for arbitrary relative fitness functions and arbitrary relative payoff functions. The algorithm may be started from an arbitrary point in the state space. Simulation by discrete variants of the dynamics in order to find a saturated equilibrium should be ruled out based on Saari [1985].

In the next section, we present our model with population dynamics. We present the saturated equilibrilum, the generalized evolutionarily stable state, and the evolutionarily stable equilibrium, and we compare these concepts with the standard fixed-point concepts. We exploit relations between evolutionary dynamics and tâtonnement processes to derive conditions which guarantee uniqueness of a saturated equilibrium, as well as convergence of all interior trajectories under the dynamical system.

Section 3.3 introduces an evolutionary model with population dynamics on one hand, and learning dynamics for all subgroups in the population on the other. We generalize the concepts of the saturated equilibrium, the generalized evolutionarily stable state, and the evolutionarily stable equilibrium. We show similar relations of these equilibrium concepts to standard fixedpoint concepts as the ones derived for the population dynamics model. We show furthermore connections to, and (perhaps more importantly) discrepancies with the standard equilibrium concepts in evolutionary game theory, the Nash equilibrium, and the evolutionarily stable equilibrium. Section 3.4 concludes.

Section 3.2 is based on Joosten [1996], Section 3.3 is based on Joosten [1995]. 


\subsection{The 'population dynamics' case}

\subsubsection{The model and equilibrium concepts}

We consider a population with $n+1$ distinguishable, strategically interacting subgroups. The population as well as the subgroups are assumed to be very large, or alternatively, the number of interactions at each point in time is very large. The interaction of the subgroups has consequences on their respective abilities to reproduce, and 'fitness' may be seen as a measure of this ability to reproduce. The fitness of a subgroup is determined by the subgroup's genetically determined behavior, and the genetically given behaviors of the other subgroups in combination with the composition of the population. Behavior of each subgroup is assumed predetermined, hence fitness can be treated as depending only on the composition of the population.

Let $I^{n+1}=\{1, \ldots, n+1\}$ and let $x=\left(x_{1}, \ldots, x_{n+1}\right)$ denote a vector of population shares. Then, $x \in S^{n}=\left\{y \in \Re^{n+1} \mid y_{j} \geq 0\right.$ for all $j \in I^{n+1}$, $\left.\sum_{i=1}^{n+1} y_{i}=1\right\}$. We suppress time-notations whenever possible. For $z, y \in$ $\Re^{n+1}$, the inner product is denoted by $z \cdot y$ and $z \leq y$ means $z_{i} \leq y_{i}$ for all $i \in I^{n+1}$. The component $j \in I^{n+1}$ of $e_{j} \in S^{n}$ is equal to one. The $(n+1)$-vector of zeroes is denoted by $0^{n+1}$. For $\alpha \in \Re$ we define $\operatorname{sign} \alpha$ by $\operatorname{sign} \alpha=1$ if $\alpha>0$, sign $\alpha=-1$ if $\alpha<0$, sign $\alpha=0$ if $\alpha=0$.

Let $E: S^{n} \rightarrow \Re^{n+1}$ be a fitness function, i.e., a function attributing to every subgroup its fitness at each state. We assume that this function is continuous and captures all relevant influences. The relative fitness function $f: S^{n} \rightarrow \Re^{n+1}$ is given by:

$$
f_{i}(x)=E_{i}(x)-\sum_{j \in I^{n+1}} x_{j} E_{j}(x) \text {, for all } i \in I^{n+1} \text { and } x \in S^{n} .
$$

Every relative fitness function is continuous and satisfies complementarity, i.e., $x \cdot f(x)=0$ for all $x \in S^{n}$. In the sequel, we treat relative fitness functions as if characterized by continuity and complementarity.

To model the evolution of the composition of the population, we consider a dynamic process represented by the following system of $n+1$ autonomous differential equations:

$$
\frac{d x}{d t}=H(x)
$$

for all $x \in S^{n}$, where $\frac{d x}{d t}=\left(\frac{d x_{1}}{d t}, \ldots, \frac{d x_{n+1}}{d t}\right)=\left(H_{1}(x), \ldots, H_{n+1}(x)\right)=H(x)$, denotes the continuous-time changes of the population shares of all subgroups. Dynamics are admissible if in Equation (3.2) 

a. $H$ is (Lipschitz) contimuous,
b. $\sum_{i=1}^{n+1} H_{i}(x)=0$ for all $x \in S^{n}$, and $H_{j}(x)=0$ whenever $x_{j}=0$.

A trajectory under the dynamics $H$ is a sequence of points, $\{x(t)\}_{t \geq 0}$, being a solution to $x(0)=x_{0} \in S^{n}$ and Equation (3.2) for all $t \geq 0$. Under (Lipschitz) continuity, existence (and uniqueness) of a solution is guaranteed (cf., e.g., Hirsch \& Smale [1974], Perko [1993]). Conditions (a) and (b) guarantee that the dynamics never leave the $n$-dimensional unit simplex.

To connect evolutionary dynamics with the payoff structure, i.e,, the relative fitness function, we introduce the concept of sign-compatibility. Evolutionary dynamics are sign-compatible if they are admissible and satisfy:

c. $\operatorname{sign} H_{i}(x)=\operatorname{sign} f_{i}(x)$ whenever $x_{i}>0$.

Evolutionary dynamics are weakly sign-compatible if they are admissible and satisfy that at each state $\mathrm{x}$, there exists at least one $i \in I^{n+1}$ such that:

$$
\text { c'. } H_{i}(x)>0 \text { implies } f_{i}(x)>0 \text {. }
$$

For sign-compatible evolutionary dynamics, the change in population share of each subgroup with positive population share corresponds in sign with its relative fitness. For weakly sign-compatible dynamics there must be at least one subgroup at each state for which this holds.

Sign-compatible dynamics are closely related to sign-preserving dynamics (Nachbar [1990]), and slightly less general than SPS-dynamics (Ritzberger \& Weibull [1995]). Sign-compatible dynamics are weakly compatible (Friedman [1991]), moreover they are myopic adjustment dynamics (Swinkels [1993]). Aggregate monotonic dynamics (Samuelson \& Zhang [1992]) are sign-compatible (Ritzberger \& Weibull [1993]). Myopic adjustment dynamics (Swinkels [1993]), best-response dynamics (Gilboa \& Matsui [1991], Matsui [1992]), and continuous fictitious-play dynamics (e.g., Rosenmüller [1971]) are weaklycompatible, not sign-compatible.

The state $y \in S^{n}$ is a fixed point if $H(y)=0^{n+1}$. A fixed point is a limit point if there exists at least one trajectory that did not start in it, converges to it. A fixed point is stable if any trajectory starting sufficiently close to it remains nearby forever. A fixed point is unstable if it is not stable. A stable fixed point is asymptotically stable if it is a limit point 
of all trajectories starting nearby. For formal definitions we refer to Hirsch \& Smale [1974].

The state $y \in S^{n}$ is a saturated equilibrium if $f(y) \leq 0^{n+1}$ (Hofbauer \& Sigmund [1988]). A saturated equilibrium $y$ is strict if $f_{j}(y)=0$ for precisely one $j \in I^{n+1}$. At a saturated equilibrium each subgroup with positive population share has highest fitness. Any subgroup with below-average-fitness has population share equal to zero. For symmetric evolutionary matrix games with inheritable fixed pure strategies, each (strict) Nash equilibrium corresponds with a (strict) saturated equilibrium. The following lists relations between concepts. Proofs can be found in Section 3.5, the appendix to this chapter.

Proposition $3.1 \quad$ (1) There exists at least one saturated equilibrium; every strict saturated equilibrium is a vertex.

(2) For weakly sign-compatible dynamics it holds that:

a. Each saturated equilibrium is a fixed point;

b. Each strict saturated equilibrium is asymptotically stable.

(3) For sign-compatible dynamics it holds that:

a. Every stable fixed point is a saturated equilibrium;

b. Every limit point of an interior trajectory is a saturated equilibrium.

A fixed point $y \in S^{n}$, is defined to be an evolutionarily stable equilibrium if there exists an open neighborhood $U \subset S^{n}$ of $y$ satisfying

$$
(y-x) \cdot H(x)>0 \text { for all } x \in U \backslash\{y\} .
$$

A fixed point $y \in S^{n}$, is defined to be a generalized evolutionarily stable state if there exists an open neighborhood $U \subset S^{n}$ of $y$ satisfying.

$$
(y-x) \cdot f(x)>0 \text { for all } x \in U \backslash\{y\} .
$$

The former concept is new in evolutionary modelling as far as we know. The latter one generalizes the evolutionarily stable strategy (cf. Maynard Smith [1982], Hofbauer \& Sigmund [1988]). For symmetric evolutionary matrix 
games with inheritable fixed strategies each evolutionarily stable strategy corresponds with a generalized evolutionarily stable state.

If Equation (3.3) or Equation (3.4) holds for an open neighborhood of a saturated equilibrium, then no other saturated equilibrium exists in this neighborhood. For a population consisting of at most two subgroups, the asymptotically stable fixed points, the evolutionarily stable equilibria, and generalized evolutionarily stable states coincide for all weakly compatible dynamics.

Given some relative fitness function and admissible dynamics, let $S S A T$, ESE, ASFP, SFP, GESS, SAT, LP int and FP denote the sets of all strict saturated equilibria, evolutionarily stable equilibria, asymptotically stable fixed points, stable fixed points, generalized evolutionarily stable states, saturated equilibria, limit points of interior trajectories, and fixed points, respectively. Connections between these sets are summarized in the following.

Proposition 3.2 For the sets given above, it holds that:

(1) $S S A T \subseteq G E S S \subseteq S A T$;

(2) For weakly sign-compatible dynamics:

$S S A T \subseteq A S F P ; E S E \subseteq A S F P ; S A T \subseteq F P ; A S F P \subseteq L P_{\text {int }} \subseteq F P$;

(3) For sign-compatible dynamics :

$S S A T \subseteq E S E \subseteq A S F P \subseteq S F P \subseteq S A T \subseteq F P$

$A S F P \subseteq L P_{\text {int }} \subseteq S A T$.

The proof of Proposition 3.2 implies that the Euclidean distance is a Lyapunov function near an evolutionarily stable equilibrium. Hence, we have the following.

Corollary 3.3 The Euclidean distance to an evolutionarily stable equilibrium decreases monotonically in time along any trajectory starting sufficiently close to it.

Corollary 3.3 is sufficient for asymptotic stability, but it is not a necessary condition. It may happen that trajectories which start near an asymptotically stable fixed point, move quite far away from it before converging. Hence, evolutionary stability is a more stringent requirement than asymptotic stability. Weissing [1990] makes a similar distinction between evolutionary stability and asymptotic stability. 
An advantage of the definition of evolutionary stability with Equation (3.3) is that it immediately implies asymptotic dynamic stability for the dynamics at hand, as well as monotonic convergence of all trajectories sufficiently nearby. Furthermore, the concept is applicable to all (weakly signcompatible) dynamics. A disadvantage is that the dynamics must be known to establish the status of a saturated equilibrium with respect to this type of evolutionary stability. Often, the payoffs on strategical interactions are better known than how to translate their effects into changes in the composition of the population. It should be noted that a saturated equilibrium for given (weakly) sign-compatible dynamics may be an evolutionarily stable equilibrium, but not for other dynamics within this class (see also Ritzberger \& Weibull [1995]).

Except for the strict saturated equilibrium, discrepancies exist between static and dynamic evolutionary stability in general. One way of dealing with these discrepancies is to follow Friedman [1991], who defines all asymptotically stable fixed points of evolutionary dynamics as 'evolutionary equilibria'. Hence, in our terminology Friedman has:

$$
S S A T \subseteq E E=A S F P \subseteq S F P \subseteq S A T \subseteq F P,
$$

where $E E$ is the set of all evolutionary equilibria. Gilboa \& Matsui [1991], Matsui [1992] define (set-valued) equilibrium concepts in terms of the dynamics as follows. A strategy is called $(\epsilon-)$ accessible from another strategy if there exists a path under (perturbed) best-response dynamics from the latter to the former. A set. of strategies is called cyclically stable if no strategy outside the set is ( $\epsilon$-)accessible from inside this set and each pair of strategies in the set is $(\epsilon-)$ accessible from one to the other and vice versa. If a cyclically stable set is a singleton, it is called a socially stable strategy. Each socially stable strategy is a saturated equilibrium in our terminology, hence Matsui [1992] has:

$$
S S S \subseteq C S S \neq S A T \text { and } S S A T \subseteq S S S=L P \subseteq S A T,
$$

where SSS is the set of socially stable strategies and CSS is the set of cyclically stable sets.

These approaches differ from the standard approach, where evolutionary stability is defined in terms of conditions on the payoff structure (cf. Maynard Smith \& Price [1973]). We regard the evolutionarily stable equilibrium as being in line with the approaches of Friedman [1991], Gilboa \& Matsui [1991], 
and Matsui [1992]. The generalized evolutionarily stable state is in line with Maynard Smith \& Price [1973]. For the replicator dynamics, we have the following.

Proposition 3.4 Every generalized evolutionarily stable state is an asymptotically stable fixed point of the replicator dynamics.

Remark 1 Let a relative fitness function $f: S^{n} \rightarrow \Re^{n+1}$ be given by:

$f_{k}(x)=s^{k} A\left(\sum_{j=1}^{n+1} x_{j} s^{j}\right)-\left(\sum_{j=1}^{n+1} x_{j} s^{j}\right) A\left(\sum_{j=1}^{n+1} x_{j} s^{j}\right)$ for all $x \in S^{n}, k \in I^{n+1}$,

where $A$ is an $(m+1) \times(m+1)$-matrix, and $s^{k} \in S^{m}$ is the fixed inheritable strategy of subgroup $k \in I^{n+1}$. Let $C=\left[s^{1}, s^{2}, \ldots, s^{n+1}\right]$ be the $(m+1) \times(n+1)$ matrix obtained by taking the strategy $s^{1}, \ldots, s^{n+1}$ as its $n+1$ columns. Then, for $y \in S^{n}$ the following two statements are equivalent:

- $y$ is a saturated equilibrium with respect to $f$;

- $(y, y)$ is a Nash equilibrium in the symmetric bi-matrix game $\left(C^{\top} A C, C^{\top} A^{\top} C\right)$.

See also Bomze \& Van Damme [1992].

\subsubsection{Evolutionary dynamics and tâtonnements}

Mathematical analogies exist between concepts in evolutionary modelling and concepts in general equilibrium theory. Sonnenschein [1972, 1973], Mantel [1974], and Debreu [1974] show that any function satisfying continuity and complementarity can be an excess demand function for a pure exchange economy with prices normalized to the unit simplex, on an arbitrarily large subset of the interior of this unit simplex. Hence, a relative fitness function may be analyzed as an excess demand function on the unit simplex. Further analogies follow from there. A Walras equilibrium corresponds with a saturated equilibrium and weakly sign-compatible dynamics correspond with tâtonnement processes (cf. Uzawa [1961]). For an overview on general equilibrium theory, we refer to, e.g., Arrow \& Hahn [1971].

A saturated equilibrium is called regular if the Jacobian matrix of the relative fitness function at this point exists and if the determinant of the $n \times n$ submatrix of the Jacobian obtained by deleting the last row and column, is 
nonzero. If each saturated equilibrium is regular, then their number is finite (Debreu [1970]) and odd (Dierker [1972], Hofbauer \& Sigmund [1988]). The relative fitness function $f$ is strongly cooperative (competitive) ${ }^{1}$ if it is differentiable and satisfies for all interior states $x$ and all $i \neq j, i, j \in I^{n+1}$

$$
\frac{\partial f_{i}(x)}{\partial x_{j}}>(<) 0 .
$$

The interpretation of Eq. (3.5) is straightforward: in the strongly cooperative (competitive) case if the population share of one subgroup increases, then the relative fitnesses of all other subgroups increase (decrease). The conditions for strong cooperativeness and gross substitutability are mathematically equivalent, see also Arrow et al. [1959], where it is shown that gross substitutability of an excess demand function implies uniqueness of a Walras equilibrium. Hence, the next two lemmas follow immediately.

Lemma 3.5 For a strongly cooperative (competitive) relative fitness function: if there exists an interior saturated equilibrium, then it is the unique interior saturated equilibrium.

Uzawa [1961] links conditions for iniqueness of a saturated equilibrium to asymptotic stability (complete instability) of a saturated equilibrium for signcompatible dynamics.

Lemma 3.6 For a strongly cooperative (competitive) relative fitness function: if there exists an interior saturated equilibrium, then each trajectory of sign-compatible dynamics from the interior of $S^{n}$ converges to (moves away from) it.

In Uzawa's proof, sign-compatibility takes such a prominent role that there is little hope to generalize Lemma 3.6 for weakly sign-compatible dynamics. Uniqueness of an interior saturated equilibrium need not hold, if strong cooperativeness (competitiveness) is slightly weakened to $\frac{\partial f_{i}(x)}{\partial x_{j}} \geq(\leq) 0$ for all $i \neq j$ and for all $x \in S^{n}$. However, all trajectories for sign-compatible dynamics starting in the interior of $S^{n}$ converge to some fixed point (cf. Uzawa [1961]).

\footnotetext{
${ }^{1}$ Hofbauer \& Sigmund [1988] use the terms cooperative and competitive for slightly different evolutionary systems. However, for the replicator dynamics examined by Hofbauer \& Sigmund, our Equation (3.5) is equivalent to their definition.
} 
For sign-compatible dynamics a necessary and sufficient condition for permanence is that the relative fitness is positive for any subgroup whose population share is close to zero. Permanence implies that no boundary saturated equilibrium is reached by any interior trajectory, and a uniform lower bound exists for the population share of all subgroups in the long run (cf. Hofbauer \& Sigmund [1988]). If a strongly cooperative system is permanent, then there exists one saturated equilibrium which is an interior state and a global attractor for the entire interior of the state space.

The problem of finding a saturated equilibrium is known in the literature as a nonlinear complementarity problem. For symmetric evolutionary bimatrix games Jansen's algorithm [1987] may be used, and its solution may give answers on the stability for the replicator dynamics. Van den Elzen \& Talman [1991] give an algorithm to find a perfect. Nash equilibrium for bimatrix games, hence it may be used to compute a saturated equilibrium for asymmetric evolutionary bi-matrix games. For nonlinear fitness functions, we must turn to more powerful methods, e.g., Doup et al. [1987]. Harker \& Pang [1990] list additional conditions which may be translated to conditions on evolutionary systems which guarantee uniqueness and stability of saturated equilibria, and may simultaneously facilitate the computation of a saturated equilibrium.

Simulation of continuous-time dynamics by a discrete approximation in order to find fixed points of dynamics involves certain caveats. Saari [1985] implies that the (informational) requirements necessary to guarantee convergence to a saturated equilibrium are generally not met by evolutionary dynamics used in simulations. Pseudo-convergence (cf., e.g., Nachbar [1992]) is an annoying problem in simulation: for long periods the dynamics appear to converge to a state which is not a limit point of the simulated dynamics. Cycling, i.e., a sequence of points being repeated an infinite number of times, is another common pitfall in simulation. Simulated dynamics may cycle, while the real dynamics do not. For evolutionary games where continuous-time dynamics admit stable limit cycles only for populations with at least four subgroups (cf. Zeeman [1980], Hofbaner [1981a]), Weissing [1990] shows existence of stable limit cycles already for populations with three subgroups for discrete dynamics. Even worse, Weissing [1990] shows that for continuous dynamics converging to an interior fixed point, the discrete analogue may spiral outward (Dekel \& Scotchmer [1992], Cabrales \& Sobel [1992]).

For higher-dimensional models complexity problems increase in general, and existence of strange attractors, or even chaos must be anticipated for the 
continuous-time dynamics, and increasingly so for discrete-time dynamics. Little is known about the behavior of discrete approximations of continuous dynamics in the presence of strange attractors in general, and even less is known about the relation between the qualitative behaviors of continuous dynamics and their discrete approximations.

\subsection{Population dynamics and myopic learn- ing}

\subsubsection{The model}

We consider a population with $n+1$ distinguishable, interacting subgroups, where $n$ is a non-negative integer. The population and its subgroups are assumed to consist of very large numbers of randomly mixing boundedly rational individuals, who can react only to the current strategic environment. Subgroup $i$ is assumed to have $m_{i}+1$ actions available with obviously $m_{i}$ a nonnegative integer.

The state space is the Cartesian product, of one $n$-dimensional unit simplex and $n+1$ umit simplices of (possibly) different dimension, and will be denoted by

$$
\Xi=S^{n} \times \prod_{i=1}^{n+1} S^{m_{i}} .
$$

An element of $\Xi$ is denoted by an ordered pair, say $(x, s)$, where $x \in S^{n}$ is an $(n+1)$-vector of population shares, and $s=\left(s^{1}, \ldots, s^{n+1}\right) \in \prod_{i=1}^{n+1} S^{m_{i}}$ is the Cartesian product of $n+1$ vectors representing the strategies employed by the $n+1$ subgroups. Obviously, the composition of the population over its $n+1$ subgroups, is characterized by the vector $x \in S^{n}$. The strategy $s^{i} \in S^{m_{i}}$, for $i \in I^{n+1}$, is the (possibly) mixed strategy used by subgroup $i$. Time notations are suppressed when confusion is unlikely. Let $(x, s),(y, u) \in \Xi$, and $p$ be a positive integer, then the p-distance between $(x, s)$ and $(y, u)$ is given by

$$
d_{p}((x, s),(y, u))=\left(\sum_{i=1}^{n+1}\left|x_{i}-y_{i}\right|^{p}+\sum_{i=\mathbb{l}}^{n+1} \sum_{h=1}^{m_{i}+1}\left|s_{h}^{i}-u_{h}^{i}\right|^{p}\right)^{\frac{1}{p}} .
$$

We refer to the 2 -distance as the Euclidean distance, and. we define $d_{\infty}(.,)=$. $\lim _{p \rightarrow \infty} d_{p}(.,$.$) . The j$-th unit vector in $\Re^{p+1}$, is denoted by $e_{j}$, and the zerovector is denoted by $0^{p+1}$. 
The fitness function $E: \Xi \rightarrow \Re^{n+1}$ is a continuous function attributing to every subgroup in the population, its fitness for every state. The fitness of a subgroup can be regarded as its (expected) average number of offspring, or alternatively, as a measure of potential to reproduce or grow. The relative fitness function (Joosten [1996]) is the function $f: \Xi \rightarrow \Re^{n+1}$ given by

$$
f_{i}(x, s)=E_{i}(x, s)-\sum_{j=1}^{n+1} x_{j} E_{j}(x, s) \text { for all }(x, s) \in \Xi, i \in I^{n+1} .
$$

Let $\pi^{i}: \Xi \rightarrow \Re^{m_{i}+1}$, for each $i \in I^{n+1}$, be a continuous payoff function, where the $h$-th component of $\pi^{i}(x, s)$ denotes subgroup $i$ 's payoff on pure action $h \in I^{m_{i}+1}$ at state $(x, s) \in \Xi$. Then, for each subgroup $i \in I^{n+1}$, the relative payoff function $g^{i}: \Xi \rightarrow \Re^{m_{1}+1}$ is given by

$$
g_{h}^{i}(x, s)=\pi_{h}^{i}(x, s)-\sum_{k=1}^{m_{i}+1} s_{h}^{i} \pi_{h}^{i}(x, s) \text { for all }(x, s) \in \Xi, h \in I^{m_{i}+1} .
$$

Note that for all $(x, s) \in \Xi: x \cdot f(x, s)=0$ and $s^{i} \cdot g^{i}(x, s)=0$ for all $i \in I^{n+1}$. Furthermore, the relative fitness function and the relative payoff functions are continuous.

Remark 2 We allow for arbitrary relative fitness functions and relative payoff functions. Connections between the payoff function and the fitness function of each subgroup should be assumed. For instance, let $(x, s) \in \Xi$, then let $s \mid w^{i}$ for all $i \in I^{n+1}$ be given by

$$
s \mid w^{i}=\left(s^{1}, \ldots, s^{i-1}, w^{i}, s^{i+1}, \ldots, s^{n+1}\right) .
$$

A plausible connection between the payoffs and the fitness of subgroup $i \in I^{n+1}$ is

$$
E_{i}(x, s)-\dot{E}_{i}\left(x, s \mid w^{i}\right)=\beta_{i}\left(s^{i} \cdot \pi^{i}(x, s)-w^{i} \cdot \pi^{i}\left(x, s \mid w^{i}\right)\right),
$$

for all $(x, s),\left(x, s \mid w^{i}\right) \in \Xi$, with given $\beta_{i}>0$. Hence, if strategy $w^{i}$ gives a higher payoff to $i \in I^{n+1}$ than $s^{i}$ does, then the strategy $w^{i}$ yields a higher fitness than strategy $s^{i}$ does. An alternative connection between payoffs and relative fitness of $i \in I^{n+1}$ is

$$
f_{i}(x, s)-f_{i}\left(x, s \mid w^{i}\right)=\beta_{i}\left(s^{i} \cdot \pi^{i}(x, s)-w^{i} \cdot \pi^{i}\left(x, s \mid w^{i}\right)\right),
$$

for all $(x, s),\left(x, s \mid w^{i}\right) \in \Xi$, with given $\beta_{i}>0$. Hence, if $w^{i}$ improves subgroup $i$ 's payoffs relative to $s^{i}$, then its relative fitness increases. Note that $(\aleph)$ does

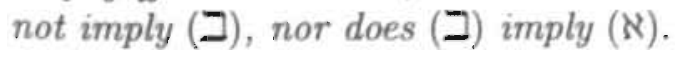


To model the evolution of the composition of the population over its subgroups, we consider a dynamic process represented by the following $(n+1)$ system of autonomous differential equations:

$$
\frac{d x}{d t}=H(x, s) \text { for all }(x, s) \in \Xi,
$$

where $H: \Xi \rightarrow \Re^{n+1}$, and $\frac{d x}{d t}=\left(\frac{d x_{1}}{d t}, \ldots, \frac{d x_{n+1}}{d t}\right)$.

To model the evolution of the strategies employed by the $n+1$ subgroups, we consider a dynamic process represented by the following $\left(\sum_{i=1}^{n+1}\left(m_{i}+1\right)\right)$ system of autonomous differential equations:

$$
\frac{d s^{i}}{d t}=G^{i}(x, s) \text { for all }(x, s) \in \Xi, i \in I^{n+1},
$$

where $G: \Xi \rightarrow \Re^{m_{i}+1}$, and $\frac{d s^{i}}{d t}=\left(\frac{d s_{1}^{i}}{d t}, \ldots, \frac{d s_{m_{i}+1}^{i}}{d t}\right)$.

The evolutionary model consists of two subsystems determined by Equations (3.8) and (3.9), respectively. The subsystem determined by Equation (3.8) is called the population dynamics in the sequel, and the one determined by Equation (3.9) is called the learning dynamics. We assume the subsystems to be interactive. Changes in the composition of the population may change the strategic environment, as may changes in the strategies used by the subgroups. The influence of one individual on the strategic environment is assumed negligeable in the short rum.

The evolutionary dynamics represented by Equations (3.8) and (3.9) are admissible if:
a. $H$ and $G^{i}$, for all $i \in I^{n+1}$, are (Lipschitz) continuous on $\Xi$,
b. $\sum_{i=1}^{n+1} H_{i}(x, s)=0$ and $\sum_{h=1}^{m_{j}+1} G_{h}^{j}(x, s)=0$ for all $(x, s) \in \Xi, j \in I^{n+1}$,
c. $G_{h}^{i}(x, s) \geq 0$ whenever $s_{h}^{i}=0$,
d. $H_{i}(x, s)=0$ whenever $x_{i}=0$.

A trajectory under the dynamical system described by Equations (3:8) and. (3.9) is a sequence of points, $\{(x(t), s(t))\}_{t \geq 0}$, being a solution to $(x(0), s(0))=$ $\left(x_{0}, s_{0}\right) \in \Xi$ and Equations (3.8) and (3.9) for all $t \geq 0$. Under Property (a), i.e., (Lipschitz) continuity, existence (and uniqueness) of a solution is guaranteed (cf., e.g., Hirsch \& Smale [1974]). Properties (c) and (d) are boundary 
conditions. They differ because for the population dynamics it is mandatory that extinct subgroups remain instinct (forward invariance). For learning dynamics, forward invariance seems rather strong.

To connect the dynamics to the payoff structure, i.e., the relative fitness function and the relative payoff functions, we introduce the concepts of signcompatibility and weak sign-compatibility. Dynamics given by Equations (3.8) and (3.9) are sign-compatible if they are admissible and satisfy:

$$
\begin{aligned}
& \text { e. } \operatorname{sign} H_{i}(x, s)=\operatorname{sign} f_{i}(x, s) \text { whenever } x_{i}>0 \text {, } \\
& \text { f. } \operatorname{sign} G_{h}^{i}(x, s)=\operatorname{sign} g_{h}^{i}(x, s) \text { whenever } s_{h}^{i}>0 \text {. }
\end{aligned}
$$

Dynamics in Equations (3.8) and (3.9) are weakly sign-compatible if they are admissible and satisfy for all $(x, s) \in \Xi$ :

$$
\begin{aligned}
& \text { e'. } \operatorname{sign} H_{i}(x, s)=\operatorname{sign} f_{i}(x, s)>0 \text { for at least one } i \in I^{n+1}, \\
& \text { f'. } \operatorname{sign} G_{h}^{j}(x, s)=\operatorname{sign} g_{h}^{j}(x, s)>0 \text { for at least one } h \in I^{m_{j}+1} \text {, for all } \\
& j \in I^{n+1} .
\end{aligned}
$$

In the following example, we derive population dynamics and learning dynamics step-by-step from the payoff structure of an evolutionary game.

Example 3.1 We consider a very large population consisting of two very large distinguishable, strategically interacting subgroups. Each individual in either subgroup has two actions available. Then, the state space is given by $\Xi=S^{1} \times S^{1} \times S^{1}$. Clearly, any state $\left(x, s^{1}, s^{2}\right) \in \Xi$, is characterized by the tuple $\left(x_{1}, s_{1}^{1}, s_{1}^{2}\right) \in[0,1]^{3}$. This allows us to represent the state space $\Xi$ by a cube. See for a graphical representation of the state space Figure 3.2.

To derive the payoff functions $\pi^{1}$ and $\pi^{2}$, we introduce the notion of the average strategy. If any individual in the population is matched with an individual of subgroup 1 , with a probability equal to $x_{1}$, and is matched with an individual of subgroup 2 with a probability equal to $\left(1-x_{1}\right)$, then the (expected) strategy against which each individual in the population plays, is the average strategy. Gilboa \& Matsui [1992] show that this procedure is justified if the subgroups are very large. For the state characterized by $\left(x_{1}, s_{1}^{1}, s_{1}^{2}\right) \in[0,1]^{3}$, the average strategy is given by

$$
S\left(x_{1}, s_{1}^{1}, s_{1}^{2}\right)=x_{1} s^{1}+\left(1-x_{1}\right) s^{2}=\left[\begin{array}{c}
x_{1} s_{1}^{1}+\left(1-x_{1}\right) s_{1}^{2} \\
x_{1}\left(1-s_{1}^{1}\right)+\left(1-x_{1}\right)\left(1-s_{1}^{2}\right)
\end{array}\right] .
$$


Let the following matrices

$$
A^{1}=\left[\begin{array}{ll}
0 & 2 \\
1 & 0
\end{array}\right] \text { and } A^{2}=\left[\begin{array}{ll}
0 & 1 \\
2 & 0
\end{array}\right]
$$

represent the payoffs of subgroup 1 and subgroup 2 respectively, to be interpreted as follows. If an individual in subgroup 1 uses action 1 and the population strategy is for instance equal to $\left[\frac{3}{4}, \frac{1}{4}\right]$, then his payoff is $e_{1} A^{1}\left[\begin{array}{c}\frac{3}{4} \\ \frac{1}{4}\end{array}\right]=\frac{1}{2}$. If he uses action 2 his (expected) payoff is $\frac{3}{4}$. For an individual in subgroup 2, the payoffs are $\frac{1}{4}$ on action 1 , and $\frac{3}{2}$ on action 2. Thus, the payoff functions for the both subgroups are given by

$$
\pi^{i}\left(x_{1}, s_{1}^{1}, s_{1}^{2}\right)=A^{i} S\left(x_{1}, s_{1}^{1}, s_{1}^{2}\right) \text { for all }\left(x_{1}, s_{1}^{1}, s_{1}^{2}\right) \in[0,1]^{3}, i=1,2 .
$$

Equating fitness for both subgroups to their average payoff yields

$$
E_{i}\left(x_{1}, s_{1}^{1}, s_{1}^{2}\right)=s^{i} \cdot \pi^{i}\left(x_{1}, s_{1}^{1}, s_{1}^{2}\right) \text { for all }\left(x_{1}, s_{1}^{1}, s_{1}^{2}\right) \in[0,1]^{3}, i=1,2 .
$$

Hence, the relative fitness function evaluated for $\left(x_{1}, s_{1}^{1}, s_{1}^{2}\right) \in[0,1]^{3}$, is:

$$
f\left(x_{1}, s_{1}^{1}, s_{1}^{2}\right)=\left[\begin{array}{c}
\left.\left(1-\sigma_{1}\right)\left(E_{1}, \sigma_{1}, v_{1}^{1}, v_{1}^{2}\right)-E_{1}\left(\sigma_{1}, \sigma_{1}^{1}, v_{1}^{2}\right),\right)^{\prime} \\
x_{1}\left(E_{2}\left(x_{1}, s_{1}^{1}, s_{1}^{2}\right)-E_{1}\left(x_{1}, s_{1}^{1}, s_{1}^{2}\right)\right)
\end{array}\right]^{\top} .
$$

The relative payoff functions evaluated for $\left(x_{1}, s_{1}^{1}, s_{1}^{2}\right) \in[0,1]^{3}$ are given by

$$
g^{i}\left(x_{1}, s_{1}^{1}, s_{1}^{2}\right)=\left[\begin{array}{c}
\left(1-s_{1}^{i}\right)\left(\pi_{1}^{i}\left(x_{1}, s_{1}^{1}, s_{1}^{2}\right)-\pi_{2}^{i}\left(x_{1}, s_{1}^{1}, s_{1}^{2}\right)\right) \\
s_{1}^{i}\left(\pi_{2}^{i}\left(x_{1}, s_{1}^{1}, s_{1}^{2}\right)-\pi_{1}^{i}\left(x_{1}, s_{1}^{1}, s_{1}^{2}\right)\right)
\end{array}\right], i=1,2 .
$$

The population dynamics are represented by

$$
\frac{d x_{1}}{d t}=x_{1} f_{1}\left(x_{1}, s_{1}^{1}, s_{1}^{2}\right)=-\frac{d x_{2}}{d t} .
$$

The learning dynamics for both subgroups are given by:

$$
\frac{d s_{1}^{i}}{d t}=s_{1}^{i} g_{1}^{i}\left(x_{1}, s_{1}^{1}, s_{1}^{2}\right)=-\frac{d s_{2}^{i}}{d t}, i=1,2 .
$$

Several calculations yield:

$$
\begin{aligned}
\frac{d x_{1}}{d t}= & x_{1}\left(1-x_{1}\right)\left(s_{1}^{1}-s_{1}^{2}\right)\left(2-x_{1}+3 x_{1} s_{1}^{2}-3 x_{1} s_{1}^{1}-3 s_{1}^{2}\right), \\
\frac{d s_{1}^{1}}{d t}= & s_{1}^{1}\left(1-s_{1}^{1}\right)\left(2+3 x_{1} s_{1}^{2}-3 x_{1} s_{1}^{1}-3 s_{1}^{2}\right), \\
\frac{d s_{1}^{2}}{d t}= & s_{1}^{2}\left(1-s_{1}^{2}\right)\left(1+3 x_{1} s_{1}^{2}-3 x_{1} s_{1}^{1}-3 s_{1}^{2}\right) .
\end{aligned}
$$




\subsubsection{Fixed points of the dynamical system}

In this section, we formulate fixed point concepts for the dynamical system. Several are standard and may be found in textbooks, e.g., Hirsch \& Smale [1974]. Other concepts are less standard, but relevant in the analysis of evolutionary systems. In Joosten [1996], we examine the saturated equilibrium (Hofbauer \& Sigmund [1988]) and define the evolutionarily stable equilibrium and the generalized evolutionarily stable state. We generalize these concepts to the present model.

The state $(y, u) \in \Xi$ is a fixed point if $H(y, u)=0^{n+1}$ and $G^{i}(y, u)=$ $0^{m_{i}+1}$ for all $i \in I^{n+1}$. A fixed point $(y, u)$ is a limit point if a trajectory $\{(x(t), s(t))\}_{t \geq 0}$ exists satisfying $(x(0), s(0)) \in \Xi \backslash\{(y, u)\}$ and $(y, u)=$ $\lim _{t \rightarrow \infty}(x(t), s(t))$. A fixed point $(y, u) \in \Xi$ is stable, if for any neighborhood $U \subset \Xi$ of $(y, u)$, there exists an open neighborhood $V \subset U$ of $(y, u)$, such that any trajectory starting in $V$ remains in $U$. A stable fixed point is asymptotically stable, if an open neighborhood $W$ of $(y, u)$ exists such that $(y, u)$ is a limit point for every trajectory starting in $W$.

Let $(y, u) \in \Xi$, then $u^{i}$ is a myopic best-response for subgroup $i \in I^{n+1}$, if

$$
u_{h}^{i}>0 \text { implies } g_{h}^{i}(y, u)=\max _{k \in I^{m_{i}+1}} g_{k}^{i}(y, u) .
$$

In that case, we write $u^{i} \in M B R_{i}(y, u)$. If $u^{i} \in M B R_{i}(y, u)$ for all $i \in I^{n+1}$, we use the notation $u \in M B R(y, u)$.

A state $(y, u) \in \Xi$ is a saturated equilibrium if:

$$
f(y, u) \leq 0^{n+1} \text { and } g^{i}(y, u) \leq 0^{m_{i}+1} \text { for all } i \in I^{n+1} .
$$

At a saturated equilibrium 'extinction of the less fit' holds: Each subgroup. with less than highest fitness has population share zero. Furthermore, each subgroup employs a myopic best-response. So, if $(y, u) \in \Xi$ is a saturated equilibrium, then $u \in M B R(y, u)$. A saturated equilibrium $(y, u)$ is strict if

$$
g_{h_{i}}^{i}(y, u)=0 \text { for precisely one } h_{i} \in I^{m_{i}+1} \text { for all } i \in I^{n+1} .
$$

A saturated equilibrium $(y, u)$ is monopolistic if

$$
f_{i}(y, u)=0 \text { for precisely one } i \in I^{n+1} \text {. }
$$

The following proposition summarizes the relations. 
Proposition 3.7 For the evolutionary model of this section:

(1) There exists at least one saturated equilibrium;

(2) For weakly sign-compatible dynamics it holds that:

a. Each saturated equilibrium is a fixed point;

b. Every monopolistic and strict saturated equilibrium is asymptotically stable;

(3) For sign-compatible dynamics it holds that:

a. Every stable fixed point is a saturated equilibrium;

b. Every limit point of an interior trajectory is a saturated equilibrium.

Not every trajectory converges, nor need it be that any trajectory not starting in a fixed point, converges (see Figure 3.1). Stable fixed points need not be limit points, and limit points may be unstable.

A fixed point $(y, u) \in \Xi$ is defined to be an evolutionarily stable equilibrium if an open neighborhood $U \subset \Xi$ of $(y, u)$ exists, satisfying

$$
(y-x) \cdot H(x, s)+\sum_{i=1}^{n+1}\left(u^{i}-s^{i}\right) \cdot G^{i}(x, s)>0 \text { for all }(x, s) \in U \backslash\{(y, u)\} .
$$

A fixed point $(y, u) \in \Xi$ is defined to be a generalized evolutionarily stable state if an open neighborhood $U \subset \Xi$ of $(y, u)$ exists, satisfying

$$
(y-x) \cdot f(x, s)+\sum_{i=1}^{n+1}\left(u^{i}-s^{i}\right) \cdot g^{i}(x, s)>0 \text { for all }(x, s) \in U \backslash\{(y, u)\} .
$$

For given relative fitness function and admissible dynamics, let the sets of all fixed points, saturated equilibria, monopolistic and strict saturated equilibria, stable fixed points, asymptotically stable fixed points, generalized evolutionarily stable states, and evolutionarily stable equilibria be denoted by $F P, S A T, M S S A T, S F P, A S F P, G E S S$, and ESE, respectively. Furthermore, let $L P_{\text {int }}$ denote the set of all limit points of trajectories starting in the interior of the state space. Then, the following lists the connections between these sets. 


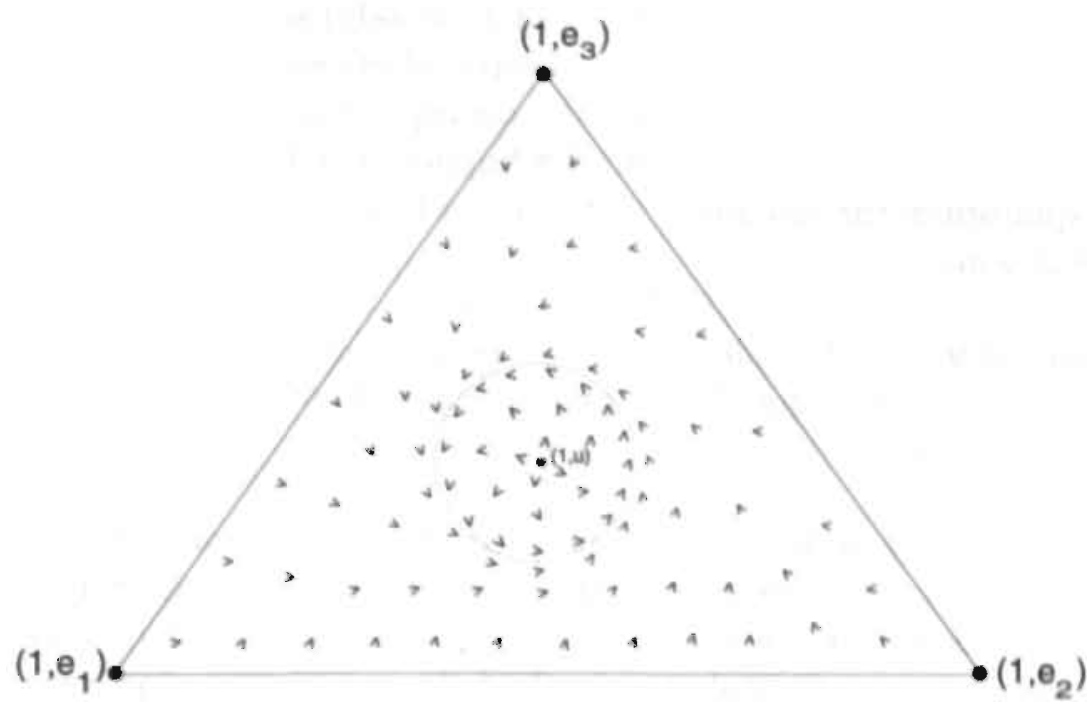

Figure 3.1: Arrowheads indicate directions of the dynamics. Note that $n=0$ and $m=2$. The state $(1, u)$ is the unique saturated equilibrium, but it is not stable. All trajectories converge to the dotted cycle.

Proposition 3.8 For the sets given above, it holds that:

(1) $M S S A T \subseteq G E S S \subseteq S A T$;

(2) For weakly sign-compatible dynamics :

$M S S A T \subseteq E S E \subseteq A S F P \subseteq S F P \subseteq F P$

$S A T \subseteq F P ; A S F P \subseteq L P_{\text {int }} \subseteq F P$;

(3) For sign-compatible dynamics :

$M S S A T \subseteq E S E \subseteq A S F P \subseteq S F P \subseteq S A T \subseteq F P ;$

$A S F P \subseteq L P_{\text {int }} \subseteq S A T$;

(4) For the replicator dynamics: GESS $\subseteq$ ASFP. 
Note that a monopolistic and strict saturated equilibrium possesses all 'desirable' dynamic convergence properties for (weakly) sign-compatible. It satisfies furthermore the conditions on the payoff structure guaranteeing evolutionary stability as formalized by the concept of generalized evolutionary stability. The proof of Proposition 3.8 implies that for each evolutionarily stable equilibrium the Euclidean distance is a Lyapunov function. This leads to the following.

Corollary 3.9 The Euclidean distance to an evolutionarily stable equilibrium decreases monotonically in time along each trajectory starting suffciently close to it.

An asymptotically stable fixed point need not be an evolutionarily stable equilibrium. For an evolutionarily stable equilibrium no trajectory starting 'sufficiently' close to it, moves away from it before converging. This is not precluded by asymptotic stability. In the following, we examine fixed points of the dynamic system for Example 3.1.

Example 3.2. We continue Example 3.1. At any fixed point $(x, s)=$ $\left(\left(x_{1}, 1-x_{1}\right),\left(s_{1}^{1}, 1-s_{1}^{1}\right),\left(s_{1}^{2}, 1-s_{1}^{2}\right)\right)$ of the dynamical system:

$$
\begin{gathered}
\frac{d x_{1}}{d t}=x_{1}\left(1-x_{1}\right)\left(s_{1}^{1}-s_{1}^{2}\right)\left(2-x_{1}+3 x_{1} s_{1}^{2}-3 x_{1} s_{1}^{1}-3 s_{1}^{2}\right)=0, \\
\frac{d s_{1}^{1}}{d t}=s_{1}^{1}\left(1-s_{1}^{1}\right)\left(2+3 x_{1} s_{1}^{2}-3 x_{1} s_{1}^{1}-3 s_{1}^{2}\right)=0, \\
\frac{d s_{1}^{2}}{d t}=s_{1}^{2}\left(1-s_{1}^{2}\right)\left(1+3 x_{1} s_{1}^{2}-3 x_{1} s_{1}^{1}-3 s_{1}^{2}\right)=0 .
\end{gathered}
$$

Several calculations show that the set of fixed points is the union of twelve compact, convex sets.

$$
\begin{aligned}
& F P=\left\{\left(e_{1}, e_{1}, e_{2}\right)\right\} \cup\left\{\left(e_{1}, e_{2}, e_{1}\right)\right\} \cup\left\{\left(e_{2}, e_{1}, e_{2}\right)\right\} \cup\left\{\left(e_{2}, e_{2}, e_{1}\right)\right\} \cup \\
& \left\{\left((\lambda, 1-\lambda), e_{1}, e_{1}\right) \mid \lambda \in[0,1]\right\} \cup\left\{\left((\lambda, 1-\lambda), e_{2}, e_{2}\right) \mid \lambda \in[0,1]\right\} \cup \\
& \left\{\left(e_{2}, e_{1},\left(\frac{1}{3}, \frac{2}{3}\right)\right)\right\} \cup\left\{\left(e_{2}, e_{2},\left(\frac{1}{3}, \frac{2}{3}\right)\right)\right\} \cup\left\{\left(e_{1},\left(\frac{2}{3}, \frac{1}{3}\right), e_{1}\right)\right\} \cup \\
& \left\{\left(e_{1},\left(\frac{2}{3}, \frac{1}{3}\right), e_{2}\right)\right\} \cup\left\{\left(\left(\frac{1}{2}, \frac{1}{2}\right), e_{1}, e_{2}\right)\right\} \cup\left\{\left(\left(\frac{1}{2}, \frac{1}{2}\right), e_{2}, e_{1}\right)\right\} .
\end{aligned}
$$




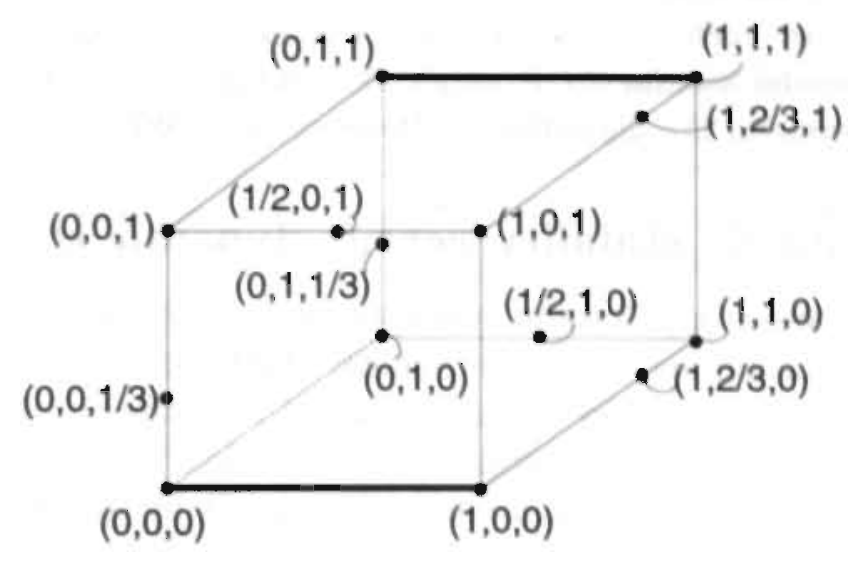

Figure 3.2: The state space represented by the unit-cube. A point $(x, y, z)$ in the unit cube, corresponds with a state $((x, 1-x),(y, 1-y),(z, 1-z))$. The sets of fixed points are denoted by heavily drawn lines and dots. The saturated equilibrium characterized by $\left(\frac{1}{2}, 1,0\right)$ is an evolutionarily stable equilibrium as well as a generalized evolutionarily stable state.

For an illustration we refer to Figure 3.2. Note that no interior fixed points exist. Furthermore, we find

$$
S A T=\left\{\left(\left(\frac{1}{2}, \frac{1}{2}\right), e_{1}, e_{2}\right)\right\} .
$$

This saturated equilibrium is strict, but not monopolistic. Moreover, it is an evolutionarily stable equilibrium, hence it is asymptotically stable. It is also a generalized evolutionarily stable state. Note that each generalized evolutionarily stable state must be an isolated saturated equilibrium, hence it is the unique generalized evolutionarily stable state. Consequenly,

$$
E S E=G E S S=A S F P=S F P=S A T .
$$

The fixed points which are not saturated equilibria, are unstable. 
Remark 3 The computations for Example 3.2 are rather tedious. The circumstance that interior fixed points do not exist in this example, simplifies calculations considerably. Finding a saturated equilibrium for higher dimensional models, or even finding an interior saturated equilibrium for a lowdimensional model similar to Example 3.2, may be quite hard. For such cases we recommend the algorithm of Doup et al. [1987].

\subsubsection{Strategic stability versus dynamic stability}

The most prominent equilibrium concepts in evolutionary game theory are the Nash equilibrium and the evolutionarily stable strategy. The evolutionarily stable strategy aims to formalize that if an invasion of one group of mutants takes place, then the 'original' strategy fares better than the 'invading' strategy in the strategic environment that arises by this invasion. Hence, the invaders are to be driven out under the selective pressures. However, the evolutionarily stable strategy is defined as a static concept in Maynard Smith \& Price [1973] and dynamics were not used until Taylor \& Jonker [1978].

The latter contribution introduced the replicator dynamics and showed that each Nash equilibrium corresponds with a fixed point of these dynamics. Moreover, each evolutionarily stable strategy in symmetric bimatrix games corresponds with an asymptotically stable fixed point of the replicator dynamics. This formalized the notion of evolutionary stability as intended by Maynard Smith and Price.

In this section, we examine the dynamical properties of strategic equilibria, i.e., the Nash equilibrium and the evolutionarily stable strategy, in our model. Let $(y, u) \in \Xi$, and recall that

$$
u \mid w^{i}=\left(u^{1}, \ldots, u^{i-1}, w^{i}, u^{i+1}, \ldots, u^{n+1}\right) \text { for } i \in I^{n+1} .
$$

Then, the strategy $x^{i} \in S^{m_{i}}$ is a best-response of subgroup $i$, if

$$
x^{i} \in \arg \max \left\{w^{i} \cdot \pi^{i}\left(y, u \mid w^{i}\right) \mid w^{i} \in S^{m_{i}}\right\} .
$$

In that case we write $x^{i} \in B R_{i}(y, u)$. We write $u \in B R(y, u)$ if $x^{i} \in B R_{i}(y, u)$ for all $i \in I^{n+1}$. The state $(y, u) \in \Xi$ is a Nash equilibrium if

$$
u \in B R(y, u) .
$$

Generalizing the concept of the evolutionarily stable strategy is not straightforward. We chose a generalized version of Theorem 9.2.8 in Van Damme 
[1991], which is originally due to Hofbaner [1981b]. The state $(y, u) \in \Xi$ is an evolutionarily stable strategy, if $u \in B R(y, u)$, and additionally, if there exists an open neighborhood $U \subset \Xi$ of $(y, u)$ satisfying

$$
u^{i} \cdot g^{i}(x, s)>0 \text { for all }(x, s) \in U \backslash\{(y, u)\} \text { whenever } s^{i} \neq u^{i} .
$$

In our model, neither a Nash equilibrium, nor an evolutionarily stable strategy need to be fixed points, since neither (3.12), nor (3.12) and (3.13) combined, imply

$$
f_{i}(y, u)=f_{j}(y, u) \text { for any pair } i, j \in I^{n+1}, i \neq j,
$$

hence it may occur that $H_{i}(y, u) \neq 0$, or $H_{j}(y, u) \neq 0$, or both.

Example 3.2 shows that a fixed point which is an evolutionarily stable strategy, need not be dynamically stable. Take namely, $\left(e_{1},\left(\frac{2}{3}, \frac{1}{3}\right), e_{2}\right)$, i.e., $x_{1}=1, s_{1}^{1}=\frac{2}{3}, s_{1}^{2}=0$, which is a fixed point and an evolutionarily stable strategy. However,

$$
f_{1}\left(1, \frac{2}{3}, 0\right)=\frac{2}{3}<\frac{4}{3}=f_{2}\left(1, \frac{2}{3}, 0\right) .
$$

Hence, it is not a saturated equilibrium, and also dynamically unstable.

Example 3.2 furthermore shows that an evolutionarily stable equilibrium need not be a Nash equilibrium, let alone an evolutionarily stable strategy. The unique evolutionarily stable equilibrium $\left(\left(\frac{1}{2}, \frac{1}{2}\right), e_{1}, e_{2}\right)$ is an asymptotically stable fixed point, hence all trajectories sufficiently nearby converge to this evolutionarily stable equilibrium. However, it may be calculated ${ }^{2}$ that $B R_{1}\left(\left(\frac{1}{2}, \frac{1}{2}\right), e_{1}, e_{2}\right)=\left\{\left(\frac{5}{6}, \frac{1}{6}\right)\right\} \neq\left\{e_{1}\right\}=M B R_{1}\left(\left(\frac{1}{2}, \frac{1}{2}\right), e_{1}, e_{2}\right)$. This implies that we have shown the following.

Proposition 3.10 Let $(y, u)$ be a saturated equilibrium, then it may occur that

$$
B R(y, u) \nsubseteq M B R(y, u) .
$$

The state $(y, u) \in \Xi$ is called a Nash* equilibrium if

$$
u \in B R(y, u) \text { and } f(y, u) \leq 0^{n+1} .
$$

${ }^{2}$ Subgroup 1's payoffs are $\frac{1}{2} s^{1} A^{1} s^{1}+\frac{1}{2} s^{1} A^{1} e_{2}=\frac{1}{2}\left(5 s_{1}^{1}-3\left(s_{1}^{1}\right)^{2}\right)$, which are maximized for $s_{1}^{1}=\frac{5}{6}$. 
The Nash* equilibrium is a 'cross-over' between a Nash equilibrium in the strategic part and a saturated equilibrium in the population dynamics part. The state $(y, u) \in \Xi$ is called an evolutionarily stable* strategy if an open neighborhood $U$ exists satisfying (3.12), (3.13) and additionally

$$
(y-x) \cdot f(x, s)>0 \text { for all }(x, s) \in U \backslash\{(y, u)) \text { whenever } x \neq y .
$$

An evolutionarily stable* equilibrium is a 'cross-over' between the evolutionarily stable strategy in the strategic part and a generalized evolutionarily stable state in the population dynamics part. An interpretation of these 'hybrid' concepts is that additional to being a Nash equilibrium or an evolutionarily stable strategy, all subgroups with positive population share have maximum fitness. All subgroups with below maximal fitness have population share zero. We now state the following, and the ensuing example will suffice as proof.

Proposition 3.11 Let $(y, u)$ be a Nash* equilibrium, then it may occur that

$$
M B R(y, u) \nsubseteq B R(y, u) .
$$

Example 3.3. Let a population consist of three distinguishable subgroups and let

$$
A^{1}=\left[\begin{array}{cc}
-1 & 13 \\
0 & 10
\end{array}\right], A^{2}=\left[\begin{array}{cc}
5 & 5 \\
0 & -10
\end{array}\right], A^{3}=\left[\begin{array}{cc}
0 & -10 \\
5 & 5
\end{array}\right],
$$

be the matrices of payoffs of individuals in subgroup 1,2 , and 3 , respectively. Consider the state $(y, u)=\left(\left[\frac{2}{10}, \frac{5}{10}, \frac{3}{10}\right],\left(e_{2}, e_{1}, e_{2}\right)\right)$. Since, the average strategy is given by $S(y, u)=\left[\frac{1}{2}, \frac{1}{2}\right]$, we have

$$
f_{i}(y, u)=u^{i} A^{i} S(y, u)-\sum_{j=1}^{3} y_{j}\left(u^{j} A^{j} S(y, u)\right)=0 \text { for } i \in I^{3} .
$$

Subgroups 2 and 3 ise strictly dominant pure strategies. For subgroup 1, it may be confirmed that $B R_{1}(y, u)=\arg \max \left\{\frac{2}{10} s^{1} A^{1} s^{1}+s^{1} A^{1}\left[\begin{array}{l}0.5 \\ 0.3\end{array}\right] \mid s^{1} \in\right.$ $\left.S^{1}\right\}=\left\{e_{2}\right\}$. This implies that $(y, u)$ is a Nash* equilibrium. However, $g^{1}(y, u)=e_{1}$, hence $(y, u)$ is not a saturated equilibrium. 
These propositions have important implications. Note that if $M B R(y, u) \nsubseteq$ $B R(y, u)$ at a Nash* equilibrium, then it is dynamically unstable for signcompatible dynamics. Furthermore, if at a saturated equilibrium $B R(y, u) \nsubseteq$ $\operatorname{MBR}(y, u)$, there may exist an interior trajectory of which this saturated equilibrium is a limit point. In that case, the system converges to a fixed point which is not a Nash equilibrium. For the evolutionarily stable* strategy, we have the following.

Proposition 3.12 Let $(y, u)$ be an evolutionarily stable* strategy, then $(y, u)$ is a generalized evolutionarily stable state.

The evolutionarily stable equilibrium in Examples 3.1 and 3.2 is a strict saturated equilibrium, but it is not a Nash equilibrium, as we have shown. Note however that any individual in subgroup 1 maximizes his payoff by using action 1. This discrepancy between rationality on 'subgroup-level' and 'individual-level', seems caused by the circumstance that no individual has a significant influence on the strategic environment. If subgroup 1 were to act as one rational player, then it would indeed switch to the best-response, and all individuals would improve. An individual's best-response is a myopic optimal decision, hence it need not be automatically optimal for the entire subgroup. A natural question is: How large must the fraction of subgroup 1 be such that, if this fraction were able to calculate a best-response to the current strategic environment, and switch to this best-response, a shift occurs away from the myopic best-response? In the Examples 3.1 ans 3.2 this fraction of subgroup 1 should be larger than $\frac{1}{2}$ for any shift away from the myopic best-response to occur ${ }^{3}$. Hence, the arguments of Matsui [1992] motivates myopic best-response rather than best-response dynamics.

\subsection{Discussion}

A crucial assumption underlying deterministic evolutionary processes is a 'large number' assumption similar to the ones we made. Formal justifications are given by e.g., Gilboa \& Matsui [1992] and Boylan [1992]. Samuelson

${ }^{3}$ Given $\lambda \in[0,1]$, the fraction of subgroup 1 which may revise its strategy at $\left(\left(\frac{1}{2}, \frac{1}{2}\right), e_{1}, e_{2}\right)$, the problem which this fraction faces, given that subgroup 2 and the rest of subgroup 1 stick to their strategies, is to find $s_{1}^{1}$ maximizing $\frac{1}{2}-\frac{1}{2} \lambda+\frac{1}{2} s_{1}^{1}+2 \lambda s_{1}^{1}-\frac{3}{2} \lambda\left(s_{1}^{1}\right)^{2}$. For $\lambda \leq \frac{1}{2}$, the solution is $s_{1}^{1}=1$, for $\frac{1}{2}<\lambda \leq 1$, the solution is $s_{1}^{1}=\frac{2}{3}+\frac{1}{6 \lambda}$. 
[1993] emphasizes that evolutionary models are sensitive to the limiting assumptions explicitly and implicitly made. Usually time and population size are taken to infinity in the limit, and perturbations in the selection process and payoffs are taken to zero in the limit. Different evolutionary dynamics may arise by taking limits in different orders.

If the population is not very large $\mathrm{e}^{4}$ for example, the matching procedure may not be approximated as implicitly assumed in this chapter. Noise may furthermore be present in the learning process or selection process. Examples are Foster \& Young [1990], Young [1993], Kandori et al. [1993], and Binmore \& Samuelson [1993]. Players may make a mistake and choose an action which is not a best response. Chance may influence natural as well as market selection. Payoffs may cause noise as well. Firstly, payoffs need not be entirely deterministic. A model where the payoffs have a stochastic component, is Fudenberg \& Harris [1992]. Secondly, it may not always be justified to equate payoffs and fitness. We may know (observe) the actual payoffs, but we do not necessarily know how they translate into fitness which drives the evolutionary process, see e.g., Binmore \& Samuelson [1993].

The distinction between the long run and the ultra-long run is important. For the long rum, the starting point and the dynamical system determine the limiting outcome of the evolutionary process. For the ultra-long run, Young [1993] and Kandori et al. [1993] predict that generically the dynamical system will be at one asymptotically stable fixed point of the dynamics with a proportion of the time going to unity as time goes to infinity. Crucial in their approach is that the asymptotically stable fixed point having the largest basin of attraction, is selected. Fudenberg \& Harris [1992] and Binmore \& Sammelson [1993] mention possible complications with respect to this prediction. We give one with a similar flavor. Imagine a 'linear' fitness landscape where attractors are pits with constant slopes, and gravity forms the evolutionary dymamics. Raridom shocks occur and generate an impulse on for instance a ball. Then, there is a one-to-one connection between the size of a basin of attraction and the difficulty for the ball to escape the pit by uphillclimbing by random shocks. In a 'nonlinear' fitness landscape, the slope of

\footnotetext{
${ }^{4}$ Often alternative assumptions are introduced for populations which are 'not very large' to replace the large number assumption, e.g., the assumption of the numbers of contests being very large. Furthermore, the unit of selection is often changed from individuals to other variables, e.g., the biomass of the subgroups is sometimes taken in mathematical ecology.
} 
the basin of attraction as well as its size matter. It may be harder to escape from a small and deep pit than from a large shallow one.

A recent development is to focus on set-valued concepts, see e.g., Gilboa \& Matsui [1991], Basu \& Weibull [1991], Matsui [1992], Ritzberger \& Weibull [1995], Gaunersdorfer \& Hofbauer [1995], and to motivate them as outcomes of evolutionary processes, i.e., inductive processes. This chapter adds a new dimension to the problem which some of these new approaches aim to solve. It suggests that the link between outcomes of evolutionary processes and game-theoretical equilibria is particularly weak when there does not exist a one-to-one relation between Nash equilibria and saturated equilibria. The reason is that evolutionary dynamics are motivated by myopic and individual strategic decisions, while dynamics leading to Nash equilibria may require a higher level of reasoning, or a large degree of coordinated action.

\subsection{Appendix}

Proof of Proposition 3.1 (1a) Standard. (Ib) and (2a) Straightforward. (2b) For the strict saturated equilibrium $e_{j}$, let $V: S^{n} \rightarrow \Re^{n+1}$ be given by $V(x)=1-x_{j}$. Then, $V(x)>0$ for $x \neq e_{j}$, and $d V(x) / d t=-H_{j}(x)$. As $H_{j}(x)>0$ for all $x$ sufficiently near $e_{j}$, asymptotic stability follows immediately. (3a) Let $y$ be a stable fixed point and suppose $j \in I^{n+1}$ satisfies $f_{j}(x)>0$. Then $V(x)=x_{j}>0$ for all $x \in$ int $S^{n}$, and $d V(x) / d t=H_{j}(x)>$ 0 , for states $x$ sufficiently near $y$. Hence, $y$ is unstable. This contradiction implies $f(y) \leq 0^{n+1}$. (3b) Straightforward.

Proof of Proposition 3.2 (1) Straightforward. (2) SSAT $\subseteq A S F P$, $S A T \subseteq F P$ follow from Prop. 3.1 ; by definition $A S F P \subseteq L P_{\text {int }} \subseteq F P$; $E S E \subseteq A S F P$ follows from: Let $y \in E S E$ and let $U \subset S^{n}$ be an open neighborhood of $y$ where Eq. (3.3) holds. Then, $V: U \rightarrow \Re$ given by $V(x)=$ $\sum_{i=1}^{n+1}\left(y_{i}-x_{i}\right)^{2}$ is a strict Lyapunov-function, since $\frac{d V(x)}{d t}=-2 \sum_{i=1}^{n+1}\left(y_{i}-\right.$ $\left.x_{i}\right) H_{i}(x)=-2(y-x) \cdot H(x)<0$ for all $x \in U \backslash\{y\}$. (3a) SSAT $\subseteq$ ESE: Let $y=e_{j} \in S S A T$, then there exists a neighborhood $U \subset S^{n}$ such that $x \in U \backslash\{y\}$ implies $\left(y_{j}-x_{j}\right) H_{j}(x)>0$ and $\left(y_{i}-x_{i}\right) H_{i}(x) \geq 0$ for all $i \neq j$. Hence, $(y-x) \cdot H(x)>0$ for all $x \in U \backslash\{y\}$. (3b) Straightforward.

Proof of Proposition 3.4 Without loss of generality we may assume that $y \in E S E \cap \operatorname{int}\left(S^{n}\right)$. Let $U \subset \operatorname{int}\left(S^{n}\right)$ where Eq. (3.4) holds. Define 
$V: U \rightarrow \Re$ by $V(x)=\sum_{i=1}^{n+1} y_{i} \ln \left(x_{i}\right)$, hence $V(x) \leq 0$ for all $x \in U \backslash\{y\}$. The replicator dynamics are given by $G_{i}(x)=x_{i} f_{i}(x)$ for all $i \in I^{n+1}$, hence: $d V(x) / d t=\sum_{i=1}^{n+1} y_{i} \frac{1}{x_{i}} G_{i}(x)=y \cdot f(x)=(y-x) \cdot f(x)>0$ for all $x \in U \backslash\{y\}$. Clearly, $V$ is maximized in $y$.

Proof of Proposition 3.7 (1) Let $F_{0}$ be the point-to-set-mapping from $\Xi$ to the subsets of $S^{n}$, satisfying for every $(x, s) \in \Xi$ :

$$
F_{0}(x, s)=\operatorname{conv}\left\{e_{j} \in S^{n} \mid f_{j}(x, s)=\max _{i \in I^{n+1}} f_{i}(x, s)\right\} .
$$

Let for each $i \in I^{n+1}, F_{i}$ be the point-to-set-mapping from $\Xi$ to the subsets of $S^{m_{i}}$, satisfying $F_{i}(x, s)=\operatorname{conv}\left\{e_{h} \in S^{m_{i}} \mid g_{h}^{i}(x, s)=\max _{k \in I^{m_{i}+1}} g_{k}^{i}(x, s)\right\}$. Let $F$ be the point-to-set-mapping from $\Xi$ to the subsets of $\Xi$, for every $(x, s) \in \Xi$ given by $F(x, s)=\prod_{i=0}^{n+1} F_{i}(x, s)$. The mappings $F_{0}, F_{1}, \ldots, F_{n+1}$ are nonempty-valued, convex-valued, compact-valued, and upper-semi-continuous by construction, hence $F$ as well. Since $\Xi$ is compact and convex, by Kakutani's fixed-point theorem $(y, u) \in \Xi$ exists, satisfying:

$$
(y, u) \in F(y, u) .
$$

Hence, $y_{j}>0$ implies $f_{j}(y, u)=\max _{i \in I^{n+1}} f_{i}(y, u)$, and $u_{h}^{i}>0$ implies $g_{h}^{i}(y, u)=\max _{k \in I^{m_{i}+1}} g_{k}^{i}(y, u)$. Let $c_{0}=\max _{i \in I^{n+1}} f_{i}(y, u)$, and let. for all $i \in I^{n+1}, c_{i}=\max _{k \in I^{m_{i}+1}} g_{k}^{i}(y, u)$. Since $(y, u) \in F(y, u)$, it follows that

$$
\sum_{i=1}^{n+1} y_{i} f_{i}(y, u)=c_{0} \text {, and } \sum_{k=1}^{m_{i}+1} u_{k}^{i} g_{k}^{i}(y, u)=c_{i} \text { for all } i \in I^{n+1} \text {. }
$$

From $y \cdot f(y, u)=0$, and $u^{i} \cdot g^{i}(y, u)=0$ for all $i \in I^{n+1}$, it follows that $c_{0}=c_{i}=0$ for all $i \in I^{n+1}$. Hence, $f(y, u) \leq 0^{n+1}$, and $g^{i}(y, u) \leq 0^{m_{i}+1}$ for all $i \in I^{n+1}$.

(2a) Straightforward. (2b) Let $(y, u)$ be a monopolistic and strict saturated equilibrium. Then, there exist one $i^{*} \in I^{n+1}$ and for each $i \in I^{n+1}$, one $j_{i}^{*} \in I^{m_{i}+1}$, satisfying $y=e_{i}$ and $u^{i}=e_{j_{i}^{*}}$. We show that $V: \Xi \rightarrow \Re$ given by

$$
V(x, s)=1-x_{i} \cdot+\sum_{i=1}^{n+1}\left(1-s_{j_{i}^{*}}^{i}\right),
$$

is a Lyapunov function on $\Xi$ near $(y, u)$. Note that $\frac{d V(x, s)}{d t}=-H_{i^{\circ}}(x, s)-$ $\sum_{i=1}^{n+1} G_{j_{i}}^{i}(x, s)<0$ for $(x, s) \rightarrow(y, u)$, since near $(y, u), f_{i^{*}}(x, s)$ is the 
unique positive component of $f(x, s)$ and similarly for every $i \in I^{n+1}$, $g_{j_{i}}^{i}(x, s)$ is the unique positive component of $g^{i}(x, s)$.

(3a) Let $(y, u) \in \Xi$ be a stable fixed point of sign-compatible dynamics and suppose $(y, u)$ is not a saturated equilibrium. Then, $i \in I^{n+1}$ exists satisfying $y_{i}=0$ and $f_{i}(y, u)>0$, or $j \in I^{n+1}$ and $k \in I^{m_{j}+1}$ exist satisfying $u_{k}^{j}=0$ and $g_{k}^{j}(y, u)>0$. Let $(y, u)$ be a stable fixed point of sign-compatible dynamics, and suppose $y_{i}=0$ and $f_{i}(y, u)>0$ for some $i \in I^{n+1}$. By continuity of $f$, $\delta>0$ and $\varepsilon>0$ exist such that $f_{i}(x, s)>0$ for all $(x, s) \in U \backslash\{(y, u)\}$ where

$$
U=\left\{(x, s) \in \Xi \mid d_{\infty}((x, s),(y, u))<\varepsilon\right\} .
$$

Let $\{(x(t), s(t))\}_{t \geq 0} \subset \Xi$ be a trajectory with $x_{i}(0)>0$. So, $(x(t), s(t)) \in U$ implies $H_{i}(x(t), s(t))>0$ for all $t \geq 0$. Then, there exists $t^{*}>0$ such that

$$
\max _{j \in I^{n+1}}\left|x_{j}\left(t^{*}\right)-y_{j}\right| \leq d_{\infty}((x, s)(y, u))=\varepsilon,
$$

implying $\left(x\left(t^{*}\right), s\left(t^{*}\right)\right) \notin U$. Hence, $\left.(x(t), s(t))\right\}_{t \geq 0}$ leaves $U$. This yields a contradiction. For the case $u_{k}^{j}=0$ and $g_{k}^{j}(y, u)>0$ for some $j \in I^{n+1}$, $k \in I^{m_{j}+1}$, a contradiction with the stability of $(y, u)$ can be proven similarly. (3b) Straightforward.

Proof of Proposition 3.8 (1) MSSAT $\subseteq$ PES is straightforward. We only prove $G E S S \subseteq S A T$. Suppose $(y, u) \in G E S S$ and $(y, u)$ is not a saturated equilibrium. Then $i \in I^{n+1}$ exists such that $f_{i}(y, u)>0$, or $j \in I^{n+1}$ and $h \in I^{m_{j}+1}$ exist such that $g_{h}^{j}(y, u)>0$.

Suppose $i \in I^{n+1}$ exists such that $f_{i}(y, u)=\varepsilon>0$. Then, take $W=\{(x, u) \in$ $\left.\Xi \mid x=(1-\lambda) y+\lambda e_{i}, \lambda \in[0,1]\right\}$. By Eq. (3.11)

$$
(y-x) \cdot f(x, u)>0 \text { for all }(x, u) \in W \cap U \backslash\{(y, u)\} .
$$

However, it follows that $\lim _{\lambda ! 0}\left(y \cdot f(x, u)-e_{i} \cdot f(x, u)\right)=-\varepsilon<0$, since $y \cdot f(y, u)=0$, and $e_{i} \cdot f(y, u)=\varepsilon>0$ by our assumption. This yields a contradiction.

Similarly, the case when $j \in I^{n+1}$ and $h \in I^{m_{j}+1}$ exist such that $g_{h}^{j}(y, u)>0$, yields a contradiction.

(2) $A S F P \subseteq S F P \subseteq F P$ holds by definition, $S A T \subseteq F P$ follows from Prop. 3.7, $L P_{\text {int }} \subseteq F P$ and $M S S A T \subseteq E S E$ are straightforward. We only prove $E S E \subseteq A S F P$. Let $(y, u) \in E S E$ and let $U \subset \Xi$ be an open neighborhood 
of $(y, u)$ such that $(3.10)$ holds for all $(x, s) \in U \backslash\{(y, u)\}$. Let $V: U \rightarrow \Re$ be defined by

$$
V(x, s)=\sum_{i=1}^{n+1}\left(y_{i}-x_{i}\right)^{2}+\sum_{i=1}^{n+1} \sum_{h=1}^{m_{i}+1}\left(u_{h}^{i}-s_{h}^{i}\right)^{2} .
$$

Obviously, $V(y, u)=0$, and $V(x, s)>0$ for all $(x, s) \in U \backslash\{(y, u)\}$. Furthermore,

$$
\begin{gathered}
\frac{d V(x, s)}{d t}=\sum_{i=1}^{n+1} \frac{\partial V(x, s)}{\partial x_{i}} \frac{d x_{i}}{d t}+\sum_{i=1}^{n+1} \sum_{h=1}^{m_{i}+1} \frac{\partial V(x, s)}{\partial s_{h}^{i}} \frac{d s_{h}^{i}}{d t}= \\
\sum_{i=1}^{n+1}-2\left(y_{i}-x_{i}\right) H_{i}(x, s)+\sum_{i=1}^{n+1} \sum_{h=1}^{m_{i}+1}-2\left(u_{h}^{i}-s_{h}^{i}\right) G_{h}^{i}(x, s)<0,
\end{gathered}
$$

for all $(x, s) \in U \backslash\{(y, u)\}$. Hence, $V$ is a strict Lyapunov function on $U$, and $(y, u) \in A S F P[\mathrm{cf}$. Hirsch and Smale (1974)].

(3) $L P_{\text {int }} \subseteq S A T$ is straightforward. We only prove $S F P \subseteq S A T$. Let $(y, u) \in S F P$ and suppose $(y, u) \notin S A T$. Then $i \in I^{n+1}$ exists such that $f_{i}(y, u)>0$, or $j \in I^{n+1}$ and $h \in I^{m_{j}+1}$ exist such that $g_{h}^{j}(y, u)>0$.

Suppose $i \in I^{n+1}$ exists such that $f_{i}(y, u)>0$. By continuity of $f$, there exist $\varepsilon_{0}, \varepsilon_{1}>0$ and a neighborhood

$$
U=\left\{(x, s) \in \Xi \mid d_{2}((x, s),(y, u))<\varepsilon_{0}\right\}
$$

satisfying $f_{i}(x, s)>\varepsilon_{1}$ for all $(x, s) \in U$. Therefore, it holds that for any trajectory $\{(x(t), s(t))\}_{t \geq 0}$ satisfying $x(0)_{i}>0$, the component $x_{i}(t)$ increases as long as $(x(t), s(t)) \in U$. Hence, there exists a $t^{*} \geq 0$ such that

$$
d_{2}\left(\left(x\left(t^{*}\right), s\left(t^{*}\right)\right),(y, u)\right)=\varepsilon_{0} .
$$

This implies that $\left(x\left(t^{*}\right), s\left(t^{*}\right)\right) \notin U$, which yields a contradiction with the stability of $(y, u)$.

The case that $j \in I^{n+1}$ and $h \in I^{m_{j}+1}$ can be proven similarly.

(4) Let $(y, u) \in G E S S$. We show that the function $V: \Xi \rightarrow \Re$ given by

$$
V(x, s)=\sum_{i=1}^{n+1} y_{i} \ln x_{i}+\sum_{i=1}^{n+1} \sum_{h=1}^{m_{i}+1} u_{h}^{i} \ln s_{h}^{i},
$$

is a Lyapunov function near $(y, u)$. Obviously, $V(x, s) \leq 0$ for all $(x, s) \in$ int $\Xi$. Note furthermore that

$$
\frac{d V(x, s)}{d t}=\sum_{i=1}^{n+1} \frac{y_{i}}{x_{i}} x_{i} f_{i}(x, s)+\sum_{i=1}^{n+1} \sum_{h=1}^{m_{i}+1} \frac{u_{h}^{i}}{s_{h}^{i}} s_{h}^{i} g_{h}^{i}(x, s)=
$$




$$
\begin{gathered}
y \cdot f(x, s)+\sum_{i=1}^{n+1} u^{i} \cdot g^{i}(x, s)= \\
(y-x) \cdot f(x, s)+\sum_{i=1}^{n+1}\left(u^{i}-s^{i}\right) \cdot g^{i}(x, s) .
\end{gathered}
$$

Hence, for the neighborhood $U$ of $(y, u)$ where Eq. (3.11) holds, we have $\frac{d V(x, s)}{d t}>0$ for $(x, s) \in U \backslash\{(y, u)\}$.

Proof of Proposition 3.12 Let $(y, u) \in E S^{*} S$, and let $U$ be the open neighborhood satisfying Equations (3.13) and (3.14). Clearly, (3.13) implies

$$
\begin{gathered}
\sum_{i=1}^{n+1} u^{i} \cdot g^{i}(x, s)>0 \text { whenever }(x, s) \in U \backslash\{(y, u)\}, s \neq u \text {, therefore } \\
\sum_{i=1}^{n+1}\left(u^{i}-s^{i}\right) \cdot g^{i}(x, s)>0 \text { for all }(x, s) \in U \backslash\{(y, u)\}, s \neq u .
\end{gathered}
$$

Combining the latter with Equation (3.14), completes the proof.

\subsection{References}

Arrow, K.J., \& F. Hahn, 1971, "General Competitive Analysis", HoldenDay, San Francisco.

Arrow, K.J., H.D. Block, \& L. Hurwicz, 1959, On the stability of the competitive equilibrium II, Econometrica 27, 82-109.

Arthur, W.B., 1990, 'Silicon Valley' locational clusters: When do increasing returns imply monopoly?, Mathematical Social Sciences 19, 235-251.

Basu, K., \& J.W. Weibull, 1991, Strategy subsets closed under rational behavior, Economics Letters 36, 141-146.

Binmore, K., \& L. Samuelson, 1993, Muddling through: Noisy equilibrium selection, mimeo. 
Björnerstedt, J. \& J.W. Weibull, 1993, Nash equilibrium and evolution by imitation, Mimeo.

Bomze, E., \& E.E.C. Van Damme, 1992, A dynamical characterization of evolutionarily stable states, Annals of Operations Research 37, 229-244.

Börgers, T., \& R. Sarin, 1995, Learning through reinforcement and replicator dynamics, mimeo.

Boylan, R.T., 1992, Laws of large numbers for dynamical systems with randomly matched individuals, Journal of Economic Theory 57, 473-504.

Cabrales, A., \& J. Sobel, 1992, On the limit points of discrete selection dynamics, Journal of Economic Theory 57, 407-419.

Crawford, V., 1991, An 'evolutionary' explanation of Van Huyck, Battalio, and Beil's experimental results on coordination, Games and Economic Behavior 3, 25-59.

Debreu, G., 1970, Economies with a finite set of equilibria, Econometrica 38, 387-392.

Debreu, G., 1974, Excess demand functions, Journal of Mathematical Economics 1, 15-23.

Dekel, E., \& S. Scotchmer, 1992, On the evolution of optimizing behavior, Journal of Economic Theory 57, 392-406.

Dierker, E., 1972, Two remarks on the number of equilibria of an economy, Econometrica 40, 951-953.

Doup, T.M., G. van der Laan, \& A.J.J. Talman, 1987, The $\left(2^{n+1}-2\right)-$ ray algorithm: a new simplicial algorithm to compute economic equilibria, Mathematical Programming 39, 241-252.

Dow, G.K., 1986, Stability analysis for profit-responsive selection mechanisms, Mathematical Social Sciences 12, 169-183. 
Elzen, A. van den, D. Talman, 1991, A procedure for finding Nash equilibria in bi-matrix games, ZOR-Methods and Models of Operations Research $35,27-43$.

Foster, D., \& H.P. Young, 1990, Stochastic evolutionary game dynamics, Theoretical Population Biology 38, 219-232.

Friedman, D., 1991, Evolutionary games in economics, Econometrica 59, $637-666$.

Fudenberg, D., \& C. Harris, 1992, Evolutionary dynamics in games with aggregate shocks, Journal of Economic Theory 57, 420-442.

Gaunersdorfer, A., \& J. Hofbauer, 1995, Fictitious play, Shapley polygons, and the replicator equation, Games and Economic Behavior 11, 279303.

Gilboa, I., \& A. Matsui, 1991, Social stability and equilibrium, Econometrica 59, 859-868.

Gilboa, I., \& A. Matsui, 1992, A model of random matching, Journal of Mathematical Economics 21, 185-187.

Harker, P.T., \& J.S. Pang, 1990, Finite-dimensional variational inequality and nonlinear complementarity problems: A survey of theory, algorithms and applications, Mathematical Programming 48, 161-220.

Hirsch, M.W., \& S. Smale, 1974, "Differential Equations, Dynamical Systems and Linear Algebra", Academic Press, New York.

Hofbauer, J., 1981a, On the occurrence of limit cycles in the Volterra-Lotka equation, Nonlinear Analysis 5, 1003- 1007.

Hofbauer, J., 1981b, A general cooperation theorem for hypercycles, Monatshefte für Mathematik 91, 233-240.

Hofbauer, J., 1995, Stability for the best response dynamics, mimeo. 
Hofbauer, J., \& K. Sigmund, 1988, "The Theory of Evolution and Dynamical Systems", Cambridge University Press, Cambridge.

Jansen, W., 1987, A permanence theorem for replicator and Lotka-Volterra systems, Journal of Mathematical Biology 25, 411-422.

Joosten, R.A.M.G., 1996, Deterministic evolutionary dynamics: A unifying approach, Journal of Evolutionary Economics 6, 313-324.

Joosten, R.A.M.G., 1995, An evolutionary model with myopic learning, Merit RM 2/94-019.

Kandori, M., G. Mailath, \& R. Rob, 1993, Learning, mutation and long run equilibria in games, Econometrica 61, 29-56.

Mantel, R., 1974, On the characterization of aggregate excess demand, Journal of Economic Theory 7, 348-353.

Matsui, A., 1992, Best response dynamics and socially stable strategies, Journal of Economic Theory 57, 343-362.

Maynard Smith, J., 1982, "Evolution and the Theory of Games", Cambridge University Press, Cambridge.

Maynard Smith, J., \& G.A. Price, 1973, The logic of animal conflict, Nature 246, 15-18.

Metcalfe, J.S., 1994, Competition, Fisher's principle and increasing returns in the selection process, Journal of Evolutionary Economics 4, 327-346.

Miller, J.H., \& J. Andreoni, 1991, Can evolutionary dynarnics explain free riding in experiments?, mimeo.

Monderer, D., \& A. Sela, 1993, Fictitious play and no-cycling conditions, mimeo.

Monderer, D., \& L.S. Shapley, 1993a, Fictitious play property for games with identical interests, mimeo. 
Monderer, D., \& L.S. Shapley, 1993b, Potential games, mimeo.

Nachbar, J.H., 1990, 'Evolutionary' selection dynamics in games: Convergence and limit properties, International Journal of Game Theory 19, 59-89.

Nachbar, J.H., 1992, Evolution in the finitely repeated prisoner's dilemma, Journal of Economic Behavior and Organization 19, 307-326.

Nash, J., 1951, Non-cooperative games, Annals of Mathematics 54, 286-295.

Oechssler, J., 1995, An evolutionary interpretation of mixed-strategy equilibria, mimeo.

Perko, L., 1993, "Differential Equations and Dynamical Systems", SpringerVerlag, Berlin.

Ritzberger, K., \& J. Weibull, 1995, Evolutionary selection in normal form games, Econometrica 63, 1371-1399.

Robinson, J., 1951, An iterative method of solving a game, Annals of Mathematics 54, 296-301.

Rosenmüller, J. 1971, Über die Periodizitätseigenschaften spieltheoretischer Lernprozesse, Zeitschrift Warscheinlichkeitstheorie und verwandte Gebiete 17, 259-308.

Saari, D., 1985, Iterative price mechanisms, Econometrica 53, 1117-1131.

Samuelson, L., 1993, Recent advances in evolutionary economics: comments, Economics Letters 42, 313-319.

Samuelson, L., \& J. Zhang, 1992, Evolutionary stability in asymmetric games, Journal of Economic Theory 57, 363-392.

Schelling, T., 1978, "Micromotives and Macrobehavior", W.W. Norton Company, NewYork. 
Schlag, K., 1994, Why imitate, and if so, how?, Mimeo.

Silverberg, J., 1988, Modelling economic dynamics and technical change: Mathematical approaches to self-organisation and evolution, in "Technical Change and Economic Theory", G. Dosi, C. Freeman, G. Silverberg and L. Soete (eds.), Pinter Publishers, London.

Sonnenschein, H., 1972, Market excess demand functions, Econometrica 40, 549-563.

Sonnenschein, H., 1973, Do Walras' identity and continuity characterize community excess demand functions?, Journal of Economic Theory 6, 345354.

Swinkels, J., 1993, Adjustment dynamics and rational play in games, Games and Economic Behavior 5, 455-484.

Taylor, P.D. \& L.B. Jonker, 1978, Evolutionarily stable strategies and game dynamics, Mathematical Biosciences 40, 145-156.

Uzawa, H., 1961, The stability of dynamic processes, Econometrica 29, 617-631.

Van Damme, E.C.C., 1991, "Stability and Perfection of Nash Equilibria", Springer, Berlin.

Weibull, J., 1995, "Evolutionary Game Theory", M.I.T. Press, Cambridge.

Weissing, F.J., 1990, Evolutionary and dynamic stability in a class of evolutionary normal form games, in "Game Equilibrium Models I, Evolution and Game Dynamics" (R. Selten, Ed.), Springer, Berlin.

Young, H.P., 1993, The evolution of conventions, Econometrica 61, 57-84.

Zeeman, E.C., 1980, Population dynamics from game theory, in "Global Theory of Dynamical Systems", Springer, Berlin. 
Zeeman, E.C., 1981, Dynamics of the evolution of animal conflicts, Journal of Theoretical Biology 89, 249-270. 



\section{Chapter 4}

\section{Changing payoffs or action sets}

\subsection{Introduction}

A game with changing payoffs or changing action sets, is a dynamic game in which the payoffs or action sets may change from one decision moment to the next as a consequence of the actions played previously. Stochastic games, as well as differential games are, generally speaking, examples of such games. Strictly speaking, repeated games are not games with changing payoffs, as the players keep the same action sets and the payoffs do not change.

Our motivation for studying games with changing payoffs or changing action sets, comes from the idea that by (not) performing certain actions the payoffs on these actions may increase (decrease), or the set of actions available may change. This may be called learning by doing or unlearning by not doing, respectively, and can be interpreted as (un)learning how to perform a physical action. An everyday example is the stamina of a long distance runner. If the runner practices regularly, his stamina will improve. If he stops practicing, his stamina will deteriorate.

There is a rich literature on learning by doing in economics following the seminal paper of Arrow [1962], especially in the contexts of growth theory and the theory of technical change. Performing a task over and over again may lead to gains in productivity or to decreasing average costs.. Much of the knowledge gained in this manner is tacit, and difficult to codify in general. This implies that this knowledge can only be acquired by actually doing the task. If one ceases to perform a task, this may lead to unlearning, i.e., losses in productivity or increasing average costs. 
The literature on unlearning by not doing is quite sparse in growth theory and the theory of technical change. One reason may be that economic and technical change is largely unidirectional: A strategy is discontinued generally because a better alternative has become available. Unlearning leads to a deterioration of the profitability of the strategy abandoned, and learning effects will increase the benefits of the adopted strategy. If switching decisions are never reversed, unlearning effects, though present, may never become apparent. See also Arrow [1994].

In human capital theory, the relevance of unlearning effects is acknowledged. Blanchard \& Summers [1986] and Möller [1990], partly ascribe persistent unemployment to the depreciation of human capital caused by the inability of the unemployed to maintain and update their skills by working. In other words, the unemployed unlearn part of the abilities they once possessed due to their inactivity. Simultaneously, they do not have the opportunity to acquire new knowledge connected to performing their job.

Acharya [1981] attributes part of the economic problems which former colonies in sub-Saharan Africa face, to a combination of the following effects. The colonial era disrupted much of the once existing African economic infrastructure in favor of linkages with the colonial power(s). In the post-colonial era the majority of these countries were not able to learn certain skills and processes fast enough to be competitive in the world market. Meanwhile, attractive alternatives which had been available in pre-colonial times, had been unlearned by not doing in the colonial era.

We contribute several models on (un)learning by (not) doing in a gametheoretical framework. In Sections 4.2 and 4.3 , we consider infinitely repeated games where two players simultaneously and independently choose an action from their respective action sets at each point in discrete time. The special feature of the games in these sections is that any action vanishes from the action set of a player, if it has not been used by him at least once during a certain number of consecutive stages. These games are special examples of stochastic games. In Section 4.2, we consider repeated zero-sum games with vanishing actions, where the gain of one player at each stage of the play is equal to the loss of the other. For several cases we give the value and optimal strategies for the limiting average reward criterion. In Section 4.3, a similar approach is followed in a general-sum framework to show existence of a Nash equilibrium for the limiting average reward criterion in several cases. Furthermore, we show a Folk-Theorem-type result.

In Section 4.4 several differential games are analyzed. Play takes place 
in continuous time, and the players take actions in continuous time. The momentary actions determine a stream of payoffs but also influence a state variable which in turn influences the payoff function. The state variable captures effects of learning by doing and unlearning by not doing in a more gradual manner than in the preceding sections.

Section 4.2 is based on Joosten, Peters \& Thuijsman [1995], Section 4.4 is based on Joosten, Peters \& Thuijsman [1994].

\subsection{Zero-sum games with vanishing actions}

A repeated zero-sum game with vanishing actions is given by an $(m \times n)$ matrix of reals $A=\left[a_{i, j}\right]_{i=1,}^{m},{ }_{j=1}^{n}$ and by two positive natural numbers $r_{1}, r_{2}$. The game, denoted by $\left(A, r_{1}, r_{2}\right)$, is played as follows. Initially players 1 and 2 have action sets $I=\{1,2, \ldots, m\}$ and $J=\{1,2, \ldots, n\}$, respectively. Players are allowed to randomize over their actions. If action $i$ of player $k$ has not been realized during $r_{k}$ consecutive stages, then action $i$ is removed from player $k$ 's action set and play continues on a submatrix of $A$. If we use the notation $r_{k}=\infty$ for instance, we mean that player $k \in\{1,2\}$ never loses an action.

Whenever an entry $(i, j)$ is selected, player 2 has to pay the stage-payoff $a_{i, j}$ to player 1. Play continues forever and both players evaluate the infinite stream of stage payoffs $R^{t}$ at stages $t=1,2, \ldots$ as a limiting average reward given by

$$
\liminf _{T \rightarrow \infty} \frac{1}{T} \sum_{T=1}^{T} R^{\tau}
$$

The limiting average reward criterion was introduced by Gillette [1957]. Player 1 wishes to maximize his expected limiting average reward, while player 2 is minimizing the same.

A repeated zero-sum game with vanishing actions is a special type of stochastic game with finite state and action spaces. It is well-known that these games have a value, both for discounted rewards. (cf. Shapley [1953]) and for limiting average rewards (cf. Mertens \& Neyman [1981]). To compute discounted value and optimal strategies there are several algorithms available (cf. Raghavan \& Filar [1991]), so in the case of discounting there is not really a problem in solving a repeated zero-sum game with vanishing actions. On the other hand, little is known about how to solve stochastic games for the limiting average reward criterion except for some very specially structured 
subclasses of games. Generally, there are no limiting average optimal strategies and only history dependent limiting average $\epsilon$-optimal strategies $(\epsilon>0)$, cf. Blackwell \& Ferguson [1968]. Repeated zero-sum games with vanishing action do not belong to any of the subclasses of stochastic games for which solution methods are known for the limiting average reward criterion.

The value of the (one-shot) matrix game $A, \operatorname{val}(A)$, is given by:

$$
\operatorname{val}(A)=\max _{\pi \in S^{m-1}} \min _{\sigma \in S^{n-1}} \pi A \sigma=\min _{\sigma \in S^{n-1}} \max _{\pi \in S^{m-1}} \pi A \sigma .
$$

We say that the matrix game $A$ has a saddlepoint at $\left(i^{*}, j^{*}\right), i^{*} \in I, j^{*} \in J$, if

$$
a_{i, j^{*}} \leq a_{i^{*}, j^{*}} \leq a_{i^{*}, j} \text { for all } i \in I, j \in J .
$$

We finish this introductory part on zero-sum games with vanishing actions with the following result. Its proof is straightforward, and therefore omitted.

Lemma 4.1 Suppose the matrix game A has a saddle point at $\left(i^{*}, j^{*}\right)$. Then, for any $r_{1}, r_{2}$ the value $v_{r_{1}, r_{2}}$ of the $\left(r_{1}, r_{2}\right)$-restricted game $\left(A, r_{1}, r_{2}\right)$ is given by $v_{r_{1}, r_{2}}=a_{i^{*}, j^{*}}$.

\subsection{1 $\left(\mathrm{r}_{1}, \mathrm{r}_{2}\right)$-Restricted zero-sum games with $r_{1} \leq 2$ or $r_{2} \leq 2$}

We start with two elementary results. Proofs are straightforward.

Lemma 4.2 The $(1,1)$-restricted game $(A, 1,1)$ has value $v_{1,1}$ given by $v_{1,1}=$ $\operatorname{val}(A)$.

Lemma 4.3 For $r \geq 2$, the $(1, r)$-restricted game $(A, 1, r)$ has value $v_{1, r}=$ $\underline{v}=\max _{i} \min _{j} a_{i, j}$. The $(r, 1)$-restricted game $(A, r, 1)$ has value $v_{r, 1}=\bar{v}=$ $\min _{j} \max _{i} a_{i, j}$.

Now, we treat the case where one player has restriction equal to two, and the other player has the advantage of being subjected to a 'milder' restriction.

Proposition 4.4 Let $A$ be an $(m \times n)$-matrix game and let $r \geq 3$. Then, the value of the $(2, r)$-restricted game $(A, 2, r)$ is given by $v_{2, r}=\underline{v}$, and the value of the $(r, 2)$-restricted game $(A, r, 2)$ is given by $v_{r, 2}=\bar{v}$. 
Proof. We only show $v_{2, r}=\underline{v}$. Obviously, player 1 can achieve at least $\underline{v}$ in the $(2, r)$-restricted game. Player 2 can guarantee $\underline{v}$ by playing as follows without loss of generality: At stage 1 let player 2 choose action 1 and suppose that player 1 also chooses action. 1. At stage 2 let player 2 choose action 2 with $a_{1,2}=\min _{j} a_{1, j}$ and suppose that player 1 chooses action 2 as well. At stage 3 let player 2 choose action 3 with $a_{2,3}=\min _{j} a_{2, j}$. At stage 3 player 1 can only choose 1 or 2 , since he has lost all other actions. If player 1 chooses 2 (and loses 1) at stage 3, then player 2 can enforce payoff $a_{2,3}$. If player 1 chooses 1 at stage 3 , then player 2 can continue by playing actions 2 and 3 alternately, starting with action 3 at stage 4 as long as player 1 keeps both actions available. If indeed player 1 keeps both actions available, this would give payoffs $a_{2,3}$ and $a_{1,2}$ in cyclic order, yielding average payoffs of at most $\underline{v}$. If player 1 chooses action 1 (2) twice in succession, then player 2 can enforce a stage payoff of $a_{1,2}\left(a_{2,3}\right)$ from then on, hence the limiting average reward will be at most $\underline{v}$.

The following result is an important intermediate step to solve (2,2)-restricted games of arbitrary size.

Proposition 4.5 For a matrix A of size $m \times 2(2 \times n)$ the $(2,2)$-restricted game $(A, 2,2)$ has value $v_{2,2}$ given by $v_{2,2}=\frac{1}{2} \underline{y}+\frac{1}{2} \bar{v}$.

Proof. At stage 1 player 1 can put probability $\frac{1}{2}$ on a row containing the first column maximum, and probability $\frac{1}{2}$ on a row containing the second column maximum. Then, with probability $\frac{1}{2}$, the initial payoff will be at least $\bar{v}$. If this happens indeed, then player 1 can obtain an average reward of at least $\bar{v}$ because, as long as player 2 has two actions, player 1 can alternate between the two rows, each time receiving at least $\bar{v}$; if player 2 loses an action, player 1 can get the maximum of the remaining column. If the initial payoff is below $\bar{v}$, then player 1 can get at least $\underline{v}$ by choosing a maxmin action. Player 2 can start with $\left(\frac{1}{2}, \frac{1}{2}\right)$ and proceed by switching as long as player 1 has both actions available and the stage payoffs have been at most $\underline{v}$. If the payoff at stage 1 was at least $\bar{v}$, then player 2 can proceed by playing the minmax action. If player 1 has only one action available player 2 can choose the action corresponding with the minimum payoff in the remaining row. Hence, $v_{2,2}=\frac{1}{2} \underline{v}+\frac{1}{2} \bar{v}$.

We now turn to solving (2,2)-restricted games with a game matrix of size $m \times n$. So, let $A=\left[a_{i, j}\right]_{i=1}^{m}{ }_{n=1}^{n}$ be an $(m \times n)$-matrix game without 
saddle points. After two stages of play in the $(2,2)$-restricted game $(A, 2,2)$ both players lose all actions except the ones selected at the first two stages of the play. So the remaining game which the players face at stage 3 will generally have size $2 \times 2$. There is no need to consider remaining games of smaller size because the activity of choosing the same action at stages 1 and 2 (and necessarily thereafter), is always weakly dominated by the activity of choosing this action at stage 1 , and any other action at stage 2 and optimizing from stage 3 onwards.

Suppose entry $(i, j)$ has been realized at stage 1 , each player will try to optimize on the expected reward in the $(2 \times 2)$-game that remains after stage 2 . If this remaining $(2 \times 2)$-game is

$$
\left[\begin{array}{ll}
a_{i, j} & a_{i, y} \\
a_{x, j} & a_{x, y}
\end{array}\right]
$$

with $x \neq i, y \neq j$, then the players entered this game through $(i, j)$ and hence, by the previous results, they know the expected reward $w_{i x, j y}$ associated with further optimal play; depending on $a_{i, j}$ the reward will either be the maxmin $\underline{v}_{i x, j y}$ or the minmax $\bar{v}_{i x, j y}$ of this $2 \times 2$-game. Using these notations we have:

$$
w_{i x, j y}= \begin{cases}\underline{v}_{i x, j y} & \text { if } a_{i, j} \leq \underline{v}_{i x, j y} \\ \bar{v}_{i x, j y} & \text { otherwise }\end{cases}
$$

At. stage 2 , conditional on $(i, j)$ at stage 1 , the players will optimize in the $(m-1) \times(n-1)$-matrix game $\left[w_{i x, j y}\right]_{x \neq i, y \neq j}$, resulting in an expected outcome of $a_{i, j}^{*}=\operatorname{val}\left[w_{i x, j y}\right]_{x \neq i, y \neq j}$. Hence, at. stage 1 the players wish to select actions that are optimal in the matrix game $A^{*}=\left[a_{i, j}^{*}\right]_{i=1}^{m},{ }_{j=1}^{n}$, and $v_{2,2}=\operatorname{val}\left(A^{*}\right)$.

Given the above considerations we can solve the game $(A, 2,2)$ by the following algorithm.

\section{Algorithm 4.1.}

Step 1. Compute the $\left(\begin{array}{c}m \\ 2\end{array}\right) \times\left(\begin{array}{c}n \\ 2\end{array}\right)$-bimatrix $B G=\left[\underline{v}_{i x, j y}, \bar{v}_{i x, j y}\right]$ where the numbers $\underline{v}_{i x, j y}$ and $\bar{v}_{i x, j y}$ correspond to the submatrix $\left[\begin{array}{cc}a_{i, j} & a_{i, y} \\ a_{x, j} & a_{x, y}\end{array}\right]$ of $A$.

Step 2. Given $B G$ compute the $m \times n$-matrix $A^{*}$ of which entry $(i, j)$ is given by $a_{i, j}^{*}=\operatorname{val}\left[w_{i x, j y}\right]_{x \neq i, y \neq j}$. 
Step 3. Compute the matrix game value and the optimal mixed actions in $A^{*}$. Then $\operatorname{val}\left(A^{*}\right)=v_{2,2}$.

We illustrate the algorithm by the following example.

Example 4.1. Consider the (2,2)-restricted game $(A, 2,2)$ with:

$$
A=\left[\begin{array}{lll}
2 & 4 & 6 \\
3 & \frac{4}{5} & 5 \\
7 & \frac{5}{2} & 0
\end{array}\right]
$$

Then, performing Step 1 yields:

$$
B G=\begin{array}{ccc}
12 & 13 & 23 \\
12 \\
13 \\
23
\end{array}\left[\begin{array}{ccc}
2,3 & 3,3 & 4,4 \\
\frac{5}{2}, 4 & 2,6 & 4,4 \\
\frac{5}{2}, \frac{5}{2} & 3,5 & \frac{4}{5}, \frac{5}{2}
\end{array}\right],
$$

where for example entry $(23,13)$ of $B G$ may be obtained as follows:

$$
A_{23,13}=\left[\begin{array}{ll}
a_{2,1} & a_{2,3} \\
a_{3,1} & a_{3,3}
\end{array}\right]=\left[\begin{array}{ll}
3 & 5 \\
7 & 0
\end{array}\right],
$$

so $\underline{v}_{i x, j y}=3$, and $\bar{v}_{i x, j y}=5$.

Performing Step 2 yields:

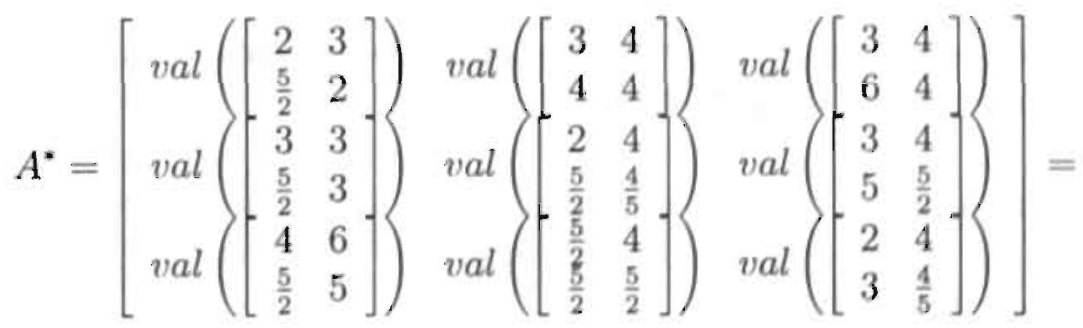

$$
\begin{aligned}
& {\left[\begin{array}{ccc}
\frac{7}{3} & 4 & 4 \\
3 & \frac{84}{37} & \frac{25}{7} \\
4 & \frac{5}{2} & \frac{52}{21}
\end{array}\right] \text {, }}
\end{aligned}
$$

where, for example, the expression $a_{3,2}^{*}=$ val $\left(\left[\begin{array}{cc}\frac{5}{2} & 4 \\ \frac{5}{2} & \frac{5}{2}\end{array}\right]\right)$ is obtained from:

$$
\left[\begin{array}{ll}
w_{31,21} & w_{31,23} \\
w_{32,21} & w_{32,23}
\end{array}\right]=\left[\begin{array}{ll}
\underline{v}_{31,21} & \underline{v}_{31,23} \\
\underline{v}_{32,21} & \underline{v}_{32,23}
\end{array}\right]=\left[\begin{array}{cc}
\frac{5}{2} & 4 \\
\frac{5}{2} & \frac{5}{2}
\end{array}\right] \text {. }
$$


The unique optimal mixed actions in $A^{*}$ are $\left(\frac{1836}{3895}, \frac{37}{3895}, \frac{2022}{3895}\right)$ for player 1 and $\left(\frac{489}{1025}, \frac{74}{1025}, \frac{462}{1025}\right)$ for player 2 , while the $(2,2)$-restricted value is given by:

$$
v_{2,2}=\operatorname{val}\left(A^{*}\right)=\frac{3285}{1025}=\frac{657}{205} .
$$

The matrix game value is $\operatorname{val}(A)=\frac{46}{13}$ and optimal mixed actions are $\left(\frac{9}{13}, 0, \frac{4}{13}\right)$ for player 1 and $\left(\frac{3}{13}, \frac{10}{13}, 0\right)$ for player 2 .

\subsection{2 (3,3)-Restricted zero-sum games}

The following result solves all $(3,3)$-restricted zero-sum games where both players have only two actions available.

Proposition 4.6 Consider the $(3,3)$-restricted game $(A, 3,3)$ with

$$
A=\left[\begin{array}{ll}
a_{1,1} & a_{1,2} \\
a_{2,1} & a_{2,2}
\end{array}\right]
$$

without saddle points. Let $\tilde{v}=\frac{1}{4}\left(a_{1,1}+a_{1,2}+a_{2,1}+a_{2,2}\right)$. Then, the value of the $(3,3)$-restricted game $(A, 3,3)$ equals $v_{3,3}=\operatorname{median}\{\underline{v}, \tilde{v}, \bar{v}\}$.

Proof. Assume that $\underline{v} \leq \tilde{v} \leq \bar{v}$ (the other cases follow similarly) and let player 1 use the following strategy:

a. (Initialization) Until a payoff of at least $\bar{v}$ is obtained, play $\left(\frac{1}{2}, \frac{1}{2}\right)$ except when it is necessary to prevent an action from vanishing; in that case choose that action;

b. If the previous payoff is at least $\bar{v}$ and player 2 has both actions available, then choose the action different from the previous one;

c. If the previous payoff is at most $\underline{v}$ and player 2 has both actions available, then choose the previous action again;

d. If player 2 has only one action left, select the action giving the highest remaining payoff. 
Now the worst thing for player 1 to happen is that play will cycle around all entries of the matrix giving average reward $\frac{1}{4}\left(a_{1,1}+a_{1,2}+a_{2,1}+a_{2,2}\right)$. This may be seen as follows. Let without loss of generality $\min \left\{a_{1,1}, a_{2,2}\right\}>$ $\max \left\{a_{1,2}, a_{2,1}\right\}$. Given the strategy of player 1 given above, player 2 may lose an action yielding at least $\bar{v}$, or the optimal play may develop according to one of the cycles:

- $\ldots a_{1,1}, a_{2,2}, a_{1,1}, a_{2,2}, \ldots$ which is a cycle of length two, yielding average payoffs of $\frac{1}{2}\left(a_{1,1}+a_{2,2}\right) \geq \bar{v} \geq \tilde{v}$;

- $\ldots a_{1,1}, a_{2,1}, a_{2,2}, a_{1,1}, a_{2,1}, a_{2,2}, \ldots$ which is a cycle of length three, yielding average payoffs of $\frac{1}{3}\left(a_{1,1}+a_{2,1}+a_{2,2}\right)$;

- $\ldots a_{1,1}, a_{1,2}, a_{2,2}, a_{1,1}, a_{1,2}, a_{2,2}, \ldots$ which is a cycle of length three, yielding average payoffs of $\frac{1}{3}\left(a_{1,1}+a_{1,2}+a_{2,2}\right)$;

- $\ldots a_{1,1}, a_{2,1}, a_{2,2}, a_{1,2}, a_{1,1}, a_{2,1}, a_{2,2}, a_{1,2}, \ldots$ which is the cycle around all entries of $A$, yielding average payoffs of $\tilde{v}$.

Let without loss of generality $a_{1,2} \leq a_{2,1}=\underline{v}$, then the worst three-cycle for player 1 , is the one yielding average payoffs of $\frac{1}{3}\left(a_{1,1}+a_{1,2}+a_{2,2}\right)$. Now suppose $\frac{1}{4}\left(a_{1,1}+a_{1,2}+a_{2,1}+a_{2,2}\right)>\frac{1}{3}\left(a_{1,1}+a_{1,2}+a_{2,2}\right)$, then the following contradiction may be derived: $\underline{v}>\frac{1}{4}\left(a_{1,1}+a_{1,2}+a_{2,1}+a_{2,2}\right)=\tilde{v}$.

Since similar arguments apply to player 2 , the proof is complete.

Proposition 4.6 is used in the proof of the following result on matrices of size $m \times 2$.

Proposition 4.7 If $A=\left[a_{i, j}\right]$ is a matrix game of size $m \times 2$, then $v_{3,3}(A)=$ $\max \left\{v_{3,3}\left(A^{\prime}\right) \mid A^{\prime}\right.$ is a $(2 \times 2)$-submatrix of $\left.A\right\}$. If $A=\left[a_{i, j}\right]$ is a matrix game of size $2 \times n$, then $v_{3,3}(A)=\min \left\{v_{3,3}\left(A^{\prime}\right) \mid A^{\prime}\right.$ is a $(2 \times 2)$-submatrix of $\left.A\right\}$.

Proof. We only prove the case where $A$ has size $m \times 2$. In each row at least one of the payoffs is at most $\underline{v}$. Suppose that player 1 uses a strategy which keeps three actions available in the long run, then he has to play these actions in a cyclical order. If player 2 has both actions alive and keeps them alive, then, every once in a while, he may repeat the same action at two consecutive stages of the play. This enables player 2 to give player 1 a payoff of at most $\underline{v}$ at all further stages. So by keeping three actions alive, player 
1 cannot expect to get more than $\underline{v}$. Hence, we may restrict ourselves to strategies of player 1 where he keeps only two actions available in the long run.

One argument in the proof above, can be extended to general $(3,3)$-restricted zero-sum games, namely that a player cannot play optimally in general by keeping three actions available forever, given that the other one only keeps two actions available. This insight is used in the following algorithm to find $v_{3,3}$ for an $(m \times n)$-game.

\section{Algorithm 4.2.}

a. Find a lower-bound and an upper bound for $v_{3,3}$ by examining what players can achieve by focussing on one action each:

$$
\underline{v} \leq v_{3,3}(A) \leq \bar{v} .
$$

b. If the previous lower-bound and upper bound are not equal, then find a better lower-bound and upper bound for $v_{3,3}$ by examining what players can achieve by focussing on two actions each:

$$
\max _{i_{1}, i_{2}} \min _{j_{1}, j_{2}} v_{3,3}\left(A_{i_{1}, i_{2}, j_{1}, j_{2}}\right) \leq v_{3,3}(A) \leq \min _{j_{1}, j_{2}} \max _{i_{1}, i_{2}} v_{3,3}\left(A_{i_{1}, i_{2}, j_{1}, j_{2}}\right)
$$

where $A_{i_{1}, i_{2}, j_{1}, j_{2}}$ is the subgame of $A$ that consists of rows $i_{1}, i_{2}$ and of columns $j_{1}, j_{2}$.

c. If the previous lower-bound and upper bound are not equal, then find a better lower-bound and upper bound for $v_{3,3}$ by examining what players can achieve by focussing on three actions each:

$$
\max _{i_{1}, i_{2}, i_{3}} \min _{j_{1}, j_{2}, j_{3}} \frac{1}{3} \sum_{t=1}^{3} a_{i_{t}, j_{t}} \leq v_{3,3}(A) \leq \min _{j_{1}, j_{2}, j_{3}} \max _{i_{1}, i_{2}, i_{3}} \frac{1}{3} \sum_{t=1}^{3} a_{i_{t}, j_{t}} .
$$

d. If the previous lower-bound and upper bound are not equal, then apply backward induction to find $v_{3,3}(A)$. This backward induction proceeds in a similar manner as for $(2,2)$-restricted games. 
Example 4.2. Consider $(A, 3,3)$ with

$$
A=\left[\begin{array}{ccc}
12 & 120 & 336 \\
252 & 0 & 36 \\
72 & 432 & 180
\end{array}\right]
$$

From this game we see that

$$
\max _{i} \min _{j} a_{i, j}=72 \leq v_{3,3} \leq 252=\min _{j} \max _{i} a_{i, j} .
$$

Looking at the $(3,3)$-values of the $(2 \times 2)$-submatrices of $A$, as given in the following matrix:

$$
B=\begin{aligned}
& 12 \\
& 13 \\
& 23
\end{aligned}\left[\begin{array}{rrr}
12 & 13 & 23 \\
96 & 159 & 120 \\
72 & 72 & 267 \\
189 & 135 & 180
\end{array}\right]
$$

we find that

$$
\max _{i_{1} i_{2}} \min _{j_{1} j_{2}} b_{i_{1} i_{2}, j_{1} j_{2}}=135 \leq v_{3,3} \leq 159=\min _{j_{1} j_{2}} \max _{i_{1} i_{2}} b_{i_{1} i_{2}, j_{1} j_{2}} .
$$

If both players keep three actions available, then they both must play in cyclical order. There are only 6 possible cycles, which are listed below. The cycle:

- $\ldots a_{1,1}, a_{2,2}, a_{3,3} \ldots$ yields an average stage payoff of 64 ;

- ... $a_{1,1}, a_{3,2}, a_{2,3} \ldots$ yields an average stage payoff of 160

- ... $a_{2,1}, a_{1,2}, a_{3,3} \ldots$ yields an average stage payoff of 184 ;

- .... $a_{2,1}, a_{3,2}, a_{1,3} \ldots$ yields an average stage payoff of 340

- ... $a_{3,1}, a_{1,2}, a_{2,3} \ldots$ yields an average stage payoff of 76 ;

- ... $a_{3,1}, a_{2,2}, a_{1,3} \ldots$ yields an average stage payoff of 136 .

Player 1 will drop action 1 , if play takes place on cycles $a_{1,1}, a_{2,2}, a_{3,3}$ or $a_{3,1}, a_{1,2}, a_{2,3}$. In this manner, he will get at least an average payoff of 135 . Player 2 will drop his action 2 if play takes place on cycles $a_{1,1}, a_{3,2}, a_{2,3}$ or $a_{2,1}, a_{1,2}, a_{3,3}$ or $a_{2,1}, a_{3,2}, a_{2,3}$. In this manner, he will have to pay at most 
159. Neither player will drop an action if play takes place on the cycle $a_{3,1}, a_{2,2}, a_{1,3}$, since this cycle yields an average stage payoff of 136 .

We can now use backward induction to find $v_{3,3}$ and optimal strategies for the players. Suppose at stage 1 entry $(2,3)$ has been selected and players continue in an optimal way. Then the next two stages will either lead to cycle $a_{1,1}, a_{3,2}, a_{2,3}$ where player 2 will drop his second action leading to an average stage payoff of 159 since player 1 will eventually drop action 3 in that case, or lead to cycle $a_{3,1}, a_{1,2}, a_{2,3}$ where player 1 will drop his first action leading to an average stage payoff of 135 since player 2 will eventually drop his second action in that case. Hence, if at stage 2 player 1 chooses action 1 and 3 with probability $\frac{1}{2}$ then the resulting average reward to him will be at least $\frac{1}{2} \cdot 159+\frac{1}{2} \cdot 135=147$. By making similar calculations for all other entries of $A$, the problem which the players face at the initial stage can be represented as

$$
A^{*}=\left[\begin{array}{ccc}
147 & 147 & 147 \frac{1}{2} \\
159 & 135 \frac{1}{2} & 147 \\
135 \frac{1}{2} & 159 & 147
\end{array}\right]
$$

By solving this matrix game we find $\operatorname{val}\left(A^{*}\right)=147 \frac{1}{6}$, and the optimal strategies in $A^{*}$ are $\left(\frac{1}{3}, \frac{1}{3}, \frac{1}{3}\right)$ for both players. By the arguments used, $v_{3,3}=\operatorname{val}\left(A^{*}\right)=147 \frac{1}{6}$, and optimal play for both players is to start each with $\left(\frac{1}{3}, \frac{1}{3}, \frac{1}{3}\right)$ followed at stage 2 by playing with probability $\frac{1}{2}$ on the actions not selected at the first stage, followed at stage 3 by selecting the action not realized at the stages 1 and 2. This is followed from stage 4 onwards by either staying in the cycle $a_{3,1}, a_{2,2}, a_{1,3}$ or else by optimizing on some $(2 \times 2)$-subgame as indicated, where player 1 has to drop his first action if a cycle is reached yielding less than 135 on average, and player 2 has to drop his second action if a cycle is reached where he has to pay more than 159 on average.

\subsubsection{On general $\left(r_{1}, r_{2}\right)$-restricted zero-sum games}

We conclude this section with several remarks, conjectures, and examples.

Proposition 4.8 For each $(m \times n)$-matrix $A$, we have

$$
\lim _{r_{1} \rightarrow \infty} \lim _{r_{2} \rightarrow \infty} v_{r_{1}, r_{2}}=\lim _{r_{2} \rightarrow \infty} \lim _{r_{1} \rightarrow \infty} v_{r_{1}, r_{2}}=\lim _{r \rightarrow \infty} v_{r, r}=\operatorname{val}(A) .
$$


Proof. Observe that for all natural numbers $r_{1}, r_{2}$ and $t$, we have the following relation:

$$
v_{r_{1}, \infty} \leq v_{r_{1}, r_{2}+t} \leq v_{r_{1}, r_{2}} \leq v_{r_{1}+t, r_{2}} \leq v_{\infty, r_{2}} .
$$

Furthermore, as $r_{1}$ increases player 1 can use his matrix game optimal mixed action for a fraction of time growing to 1, every now and then selecting pure actions in order to keep them available if necessary. Thus on the whole play his expected payoff will approach $\operatorname{val}(A)$ as $r_{1}$ increases. This implies that $\lim _{r_{1} \rightarrow \infty} v_{r_{1}, \infty}=\operatorname{val}(A)$. Similarly, $\lim _{r_{2} \rightarrow \infty} v_{\infty, r_{2}}=\operatorname{val}(A)$.

Example 4.3. For the $(2 \times 2)$-matrix

$$
A=\left[\begin{array}{ll}
1 & 3 \\
2 & 0
\end{array}\right]
$$

we have:

- The value of the one-shot matrix game $A$ is $\operatorname{val}(A)=\frac{3}{2}$. For player 1 the unique optimal mixed action is $\left(\frac{1}{2}, \frac{1}{2}\right)$, while for player 2 the unique optimal mixed action is $\left(\frac{3}{4}, \frac{1}{4}\right)$. By Lemma $4.2, v_{1,1}=\operatorname{val}(A)$;

- For $r>1$ we have: $v_{1, r}=\underline{v}=1$ and $v_{r, 1}=\bar{v}=2$.

For the case $(1, r)$, optimal strategies are the following. For player 1: Play action 1 at all stages. For player 2 : Choose the first-stage-action randomly and continue with choosing the action which minimizes player l's stage payoff from then on;

- For $r>2$ we have: $v_{2, r}=\underline{v}=1$ and $v_{r, 2}=\bar{v}=2$.

For the case $(2, r)$ optimal strategies are the following. For player 1: Play action 1 at all stages. For player 2 : Choose action 1 at the first stage, choose action 2 at the second stage, followed by:

- If the first two payoffs were 1 and 0 (resp. 2 and 3), then play both actions alternately starting with action 1 (resp. action 2) at stage 3 , until. player 1 loses an action;

- Otherwise, choose action 1 if player 1 has action 1 left, and action 2 otherwise; 
- $v_{2,2}=\frac{1}{2} \underline{y}+\frac{1}{2} \bar{v}=\frac{1}{2}(1+2)=\frac{3}{2}$. An optimal strategy for player 1 is: Start with $\left(\frac{1}{2}, \frac{1}{2}\right)$, followed by playing the actions alternately as long as payoffs 2 and 3 appear, and to play his maxmin action in the remaining game otherwise. An optimal strategy for player 2 is to start with $\left(\frac{1}{2}, \frac{1}{2}\right)$, continue with playing the actions alternately as long as payoffs 0 and 1 appear, and playing his minmax action in the remaining game otherwise;

- $v_{3,3}=\frac{1}{4}\left(a_{1,1}+a_{1,2}+a_{2,1}+a_{2,2}\right)=\frac{3}{2}$. An optimal strategy for player 1 is to start by playing $\left(\frac{1}{2}, \frac{1}{2}\right)$ followed by:

- As long as payoffs are at most 1 , play $\left(\frac{1}{2}, \frac{1}{2}\right)$, or pure if necessary to prevent an action from vanishing,

- If the previous payoff is at least 2 and player 2 has both actions. available, then choose the opposite action;

- If the previous payoff is at most 1 and player 2 has both actions available, then choose the same action again;

- If player 2 has only one action left, select the maximum payoff.

At worst for player 1, this strategy will force play to cycle around all entries of the matrix $A$ giving a limiting average reward of $\frac{3}{2}$;

- For $\left(A, r_{1}, \infty\right)$ with $r_{1} \geq 3$ we conjecture

$$
v_{r_{1}, \infty}=\frac{3}{2}-\frac{1}{2 r_{1}-1}-1 \text {, }
$$

while for player 1 it is optimal to play $\left(\frac{1}{2}, \frac{1}{2}\right)$ as long as none of his actions is about to vanish immediately, and to select such an action if it, is about to vanish. It is not hard to show that this strategy indeed guarantees the presented amount. However, we do not know a general ( $r_{1}$-dependent) strategy for player 2 to guarantee this amount. Using the algorithm of Vrieze [1981], we could verify that for many different instances of $r_{1}$, the value equals the presented amount. (Clearly, $v_{r_{1}, \infty} \leq \frac{3}{2}$ since player 2 may play $\left(\frac{3}{4}, \frac{1}{4}\right)$ at all stages.)

- For the game $\left(A, \infty, r_{2}\right)$ with $r_{2} \geq 3$ we conjecture

$$
v_{\infty, r_{2}}=\frac{3}{2}+\frac{3}{2}\left(\frac{3^{r_{2}-1}+1}{4^{r_{2}}-3^{r_{2}}-1}\right)
$$


by similar arguments as above.

We remark that $\left(r_{1}, \infty\right)$-restricted games and $\left(\infty, r_{2}\right)$-restricted games are stochastic games where player 1 or player 2 controls the transition structure (so-called single-controller stochastic games). Such games are well-known in the literature. Solutions, i.e., a value and optimal strategies, for the limiting average reward criterion can be found by solving a single linear program and its dual (cf. Vrieze [1981], Filar \& Raghavan [1984]). As

$$
v_{r_{1}, \infty} \leq v_{r_{1}, r_{2}} \leq v_{\infty, r_{2}},
$$

we may establish upper and lower bounds for the $\left(r_{1}, r_{2}\right)$-restricted value by using either algorithm.

\subsection{General-sum games with vanishing actions}

A general-sum game with vanishing actions is given by two $(m \times n)$-matrices of reals $A=\left[a_{i, j}\right]_{i=1}^{m} n_{j=1}^{n}, B=\left[b_{i, j}\right]_{i=1}^{m}, n_{j=1}^{n}$ and two natural numbers $r_{1}, r_{2}$. The game, denoted by $\left(A, B, r_{1}, r_{2}\right)$ in the sequel, is played similar to the zero-sum game. We denote the history of play until stage 1 by the empty sequence, i.e., $h^{1}=()$, and the history of play until stage $t \in\{2,3, \ldots\}$ by $h^{t}=\left(\left(i^{1}, j^{1}\right), \ldots,\left(i^{t-1}, j^{t-1}\right)\right)$ where $i^{s} \in I$ and $j^{s} \in J$ are the actions that were played at stage $s \in\{1, \ldots, t-1\}$. The set of all histories of play until stage $t \in\{1,2,3, \ldots\}$ is denoted by $H^{t}$. Given the restriction $r_{1}$, a strategy for player 1 is a function

$$
\pi: \cup_{t=1}^{\infty} H^{t} \rightarrow S^{m-1}
$$

with $\pi_{i}\left(h^{t}\right)=0$ if action $i \in I$ was not used during $r_{1}$ consecutive stages of the play until $t$. Similarly, given $r_{2}$, a strategy for player 2 is a function

$$
\sigma: \cup_{t=1}^{\infty} H^{t} \rightarrow S^{n-1},
$$

with $\sigma_{j}\left(h^{t}\right)=0$ if action $j \in J$ was not used during $r_{2}$ consecutive stages of the play until $t$. We use $\pi\left(h^{t}\right)=i$ as short-hand notation for $\pi_{i}\left(h^{t}\right)=1$ for $i \in I$.

Given $\pi$ and $\sigma$, let $R_{k}^{t}(\pi, \sigma)$ be the expected stage payoff of player $k \in$ $\{1,2\}$ at stage $t$. Play continues forever, and player $k \in\{1,2\}$ evaluates the 
infinite stream of expected stage payoffs under $\pi, \sigma$ as a limiting average reward $\gamma_{k}(\pi, \sigma)=\liminf _{T \rightarrow \infty} \frac{1}{T} \sum_{\tau=1}^{T} R_{k}^{\tau}(\pi, \sigma)$. Simultaneously and independently of each other, both players try to maximize their limiting average rewards.

Definition 4.9 The strategy combination $\left(\pi^{*}, \sigma^{*}\right)$ is a Nash equilibrium for $\left(A, B, r_{1}, r_{2}\right)$ if

- $\gamma_{1}\left(\pi^{*}, \sigma^{*}\right) \geq \gamma_{1}\left(\pi, \sigma^{*}\right)$ for all strategies $\pi$,

- $\gamma_{2}\left(\pi^{*}, \sigma^{*}\right) \geq \gamma_{2}\left(\pi^{*}, \sigma\right)$ for all strategies $\sigma$.

The strategies $\pi^{*}, \sigma^{*}$ are called (Nash) equilibrium strategies, the expected limiting average rewards, i.e., $\left(\gamma_{1}\left(\pi^{*}, \sigma^{*}\right), \gamma_{2}\left(\pi^{*}, \sigma^{*}\right)\right)$ are called the (Nash) equilibrium rewards. So, a Nash equilibrium is a pair of strategies satisfying that neither player can improve his limiting average reward by unilaterally deviating from his strategy. A Nash equilibrium $\left(\pi^{*}, \sigma^{*}\right)$ is pure if both $\pi^{*}$ and $\sigma^{*}$ are pure strategies, i.e., $\pi^{*}\left(h^{t}\right) \in I$ and $\sigma^{*}\left(h^{t}\right) \in J$ for all $t \in\{1,2, \ldots\}$.

We denote a repeated general-sum game with payoff matrices $A$ and $B$, by $(A, B, \infty, \infty)$, and a Nash equilibrium for $(A, B, \infty, \infty)$ is given by Definition 4.9 with $r_{1}=r_{2}=\infty$. We denote the (one-shot) general-sum game with payoff matrices $A$ and $B$, by $(A, B)$. The (one-shot) strategies for players 1 and 2 are denoted by $\pi(1) \in S^{m-1}$ and $\sigma(1) \in S^{n-1}$, respectively.

Definition 4.10 The strategy combination $\left(\pi^{*}(1), \sigma^{*}(1)\right)$ is a Nash equilibrium for $(A, B)$ if

$$
\begin{aligned}
& \text { - } \pi^{*}(1) A \sigma^{*}(1) \geq \pi(1) A \sigma^{*}(1) \text { for all } \pi(1) \in S^{m-1} \text {, } \\
& \text { - } \pi^{*}(1) B \sigma^{*}(1) \geq \pi^{*}(1) B \sigma(1) \text { for all } \sigma(1) \in S^{n-1} \text {. }
\end{aligned}
$$

The strategies $\pi^{*}(1), \sigma^{*}(1)$ are called (Nash) equilibrium strategies.

A Nash equilibrium for a one-shot game $(A, B)$ is called pure if both equilibrimm strategies are pure, i.e., $\pi^{*}(1) \in I$, and $\sigma^{*}(1) \in J$.

An $\epsilon$-equilibrium $(\epsilon>0)$ is a pair of strategies satisfying that neither player can improve more than $\epsilon$ by deviating unilaterally from his equilibrium strategy. As mentioned before, repeated games with vanishing actions are a special class of stochastic games. For zero-sum stochastic games, Mertens \& 
Neyman [1981] have shown the existence of the limiting average value. However, the existence of limiting average $\epsilon$-equilibria in general-sum stochastic games is still an open problem. A survey on existence-results for $\epsilon$-equilibria can be found in Thuijsman [1992]. The class of games examined in this section does not belong to any of the classes for which existence results of $\epsilon$-equilibria have been published.

For unrestricted repeated games with the limiting average reward criterion, the 'Folk Theorem' states that all feasible, individually rational outcomes may be obtained as a Nash equilibrium outcome (cf., e.g., Aumann [1981]). In the following subsection, we introduce conditions under which a parallel to this 'Folk Theorem', exists for general-sum games with vanishing actions. In Subsection 4.3.2, we show existence of a Nash equilibrium for several cases. We end this introductory part on general-sum games with vanishing actions by giving a connection between the Nash equilibria of oneshot general-sum games and the Nash equilibria of unrestricted repeated general-sum games. Then, we give a similar result for pure Nash equilibria in a one-shot game and pure Nash equilibria in a $\left(r_{1}, r_{2}\right)$-restricted repeated general-sum game.

Lemma 4.11 The following two statements are equivalent:

i. $\left(\pi^{*}(1), \sigma^{*}(1)\right)$ is a Nash equilibrium of $(A, B)$;

ii. $\left(\pi^{*}, \sigma^{*}\right)$ satisfying $\pi^{*}=\left(\pi^{*}(1), \pi^{*}(1), \ldots\right)$ and $\sigma^{*}=\left(\sigma^{*}(1), \sigma^{*}(1), \ldots\right)$, is a Nash equilibrium of $(A, B, \infty, \infty)$.

An analogous result holds for pure Nash equilibria in $(A, B)$ and pure stationary Nash equilibria in $\left(A, B, r_{1}, r_{2}\right)$, the proof is straightforward.

Proposition 4.12 The following two statements are equivalent:

i. $\left(i^{*}, j^{*}\right)$ is a Nash equilibrium for the game $(A, B)$;

ii. $\left(\pi^{*}, \sigma^{*}\right)$ with $\pi^{*}=\left(i^{*}, i^{*}, i^{*}, \ldots\right)$, and $\sigma^{*}=\left(j^{*}, j^{*}, j^{*}, \ldots\right)$, is a Nash equilibrium in $\left(A, B, r_{1}, r_{2}\right)$. 


\subsubsection{A 'Folk 'Theorem' for $\left(r_{1}, r_{2}\right)$-restricted games}

In this subsection, we derive a 'Folk Theorem' for restricted repeated generalsum games, but we will firstly focus on the Folk Theorem for unrestricted repeated general-sum games. We describe which sets of rewards in a repeated. game are feasible and which are individually' rational. Then, we give the Folk Theorem on repeated games. We proceed by showing which sets of rewards are feasible in an $\left(r_{1}, r_{2}\right)$-restricted game, and which sets of rewards are individually rational. Then, we give an analogy to the Folk Theorem for $\left(r_{1}, r_{2}\right)$-restricted games.

Consider $(A, B, \infty, \infty)$. Let $v_{A}=\max _{\pi} \min _{\sigma} \pi A \sigma$ denote the matrix game value of the zero-sum game $A$. Similarly, let $v_{B}=\max _{\sigma} \min _{\pi} \pi B \sigma$. Let $\pi_{A}^{*}, \sigma_{A}^{*}$ be such that $\pi_{A}^{*} A y \geq v_{A} \geq x A \sigma_{A}^{*}$ for all strategies $x, y$. Similarly, let $\pi_{B}^{*}, \sigma_{B}^{*}$ be such that $x B \sigma_{B}^{*} \geq v_{B} \geq \pi_{B}^{*} B y$ for all strategies $x, y$. Then, in $(A, B, \infty, \infty)$, player 1 can obtain a limiting average reward of at least $v_{A}$, by using $\pi_{A}^{*}$ at every stage of the play. Player 2 can keep player 1 's limiting average reward at $v_{A}$, by using $\sigma_{A}^{*}$ at every stage of the play. Similarly, player 2 can get a limiting average reward of at least. $v_{B}$ by using $\sigma_{B}^{*}$ at every stage. Player 1 can keep player 2's limiting average reward at $v_{B}$ by using $\pi_{B}^{*}$ at each stage.

Definition 4.13 For $(A, B, \infty, \infty)$ the set of individually rational rewards is given by:

$$
\operatorname{IRR}(A, B, \infty, \infty)=\left\{(x, y) \in \Re^{2} \mid x \geq v_{A}, y \geq v_{B}\right\} .
$$

Definition 4.14 For $(A, B, \infty, \infty)$ the set of feasible rewards is given by:

$$
F R(A, B, \infty, \infty)=\left\{(x, y) \in \Re^{2} \mid \exists(\pi, \sigma):\left(\gamma_{1}(\pi, \sigma), \gamma_{2}(\pi, \sigma)\right)=(x, y)\right\} .
$$

The following lemma is well known. We give a proof because we want to refer to it later on.

Lemma $4.15 F R(A, B, \infty, \infty) \supseteq \operatorname{conv}\left\{\left(a_{i, j}, b_{i, j}\right) \mid i \in I, j \in J\right\}$.

Proof. Take $(a, b) \in \operatorname{conv}\left\{\left(a_{i, j}, b_{i, j}\right) \mid i \in I, j \in J\right\}$. Let $\left\{\lambda_{i, j} \geq 0 \mid i \in I\right.$, $\left.j \in J, \sum_{i \in I, j \in J} \lambda_{i, j}=1\right\}$, satisfy $(a, b)=\sum_{i \in I, j \in J} \lambda_{i, j}\left(a_{i, j}, b_{i, j}\right)$.

We construct $(\pi, \sigma)$ satisfying $\left(\gamma_{1}(\pi, \sigma), \gamma_{2}(\pi, \sigma)\right)=(a, b)$. Choose $\left(i^{1}, j^{1}\right)$ arbitrarily. Define $\left(i^{t+1}, j^{t+1}\right), t \in\{1,2, \ldots\}$, recursively as follows: 
Let $f_{t}(i, j)$ be the fraction of the times the pair $(i, j)$ has occurred in the sequence $\left(\left(i^{1}, j^{1}\right), \ldots,\left(i^{t}, j^{t}\right)\right)$. Choose $\left(i^{t+1}, j^{t+1}\right)$ such that

$$
f_{t}\left(i^{t+1}, j^{t+1}\right)-\lambda_{i} t_{+1} j^{t+1}=\min _{(i, j)}\left(f_{t}(i, j)-\lambda_{i, j}\right) .
$$

It can be verified that $\lim _{t \rightarrow \infty} f_{t}(i, j)=\lambda_{i, j}$ for all $i \in I, j \in J$. With the above, define $(\pi, \sigma)$ by: $\left(\pi\left(h^{t}\right), \sigma\left(h^{t}\right)\right)=\left(i^{t}, j^{t}\right)$ for all $t \in\{1,2, \ldots\}$. Then, $\left(\gamma_{1}(\pi, \sigma), \gamma_{2}(\pi, \sigma)\right)=(a, b)$.

Every Nash equilibrium in $(A, B, \infty, \infty)$ must be feasible and yield individually rational rewards. The former statement is obvious, and the latter one follows since player 1 has a strategy of receiving at least $v_{A}$ regardless of his opponent's behavior. Similarly, player 2 has a strategy of receiving at least $v_{B}$ regardless of his opponent's behavior. So, any Nash equilibrium should give both players at least these rewards. Let $\operatorname{NER}(A, B, \infty, \infty)$ denote the set of all limiting average Nash equilibrium rewards of $(A, B, \infty, \infty)$. The following is well known.

\section{Theorem 4.16 ('Folk Theorem' on repeated games)}

$$
(\operatorname{IRR}(A, B, \infty, \infty) \cap \operatorname{FR}(A, B, \infty, \infty))=\operatorname{NER}(A, B, \infty, \infty) .
$$

Instead of giving a formal proof, we illustrate the idea by considering a small example.

Example 4.4. Consider the game $(A, B, \infty, \infty)$ with $(A, B)$ given by

$$
(A, B)=\left[\begin{array}{ll}
1,0 & 0,1 \\
0,2 & 1,0
\end{array}\right] \text {. }
$$

Note that $\pi_{A}^{*}=\left(\frac{1}{2}, \frac{1}{2}\right), \sigma_{A}^{*}=\left(\frac{1}{2}, \frac{1}{2}\right), v_{A}=\frac{1}{2}$, similarly $\pi_{B}^{*}=\left(\frac{2}{3}, \frac{1}{3}\right), \sigma_{B}^{*}=\left(\frac{1}{3}, \frac{2}{3}\right)$, and $v_{B}=\frac{2}{3}$. Then,

$$
N E R(A, B, \infty, \infty)=\left\{(x, y) \mid x \geq \frac{1}{2}, y \geq \frac{2}{3}, 2 x+y \leq 2\right\} .
$$

Take $(\bar{x}, \bar{y})=\left(\frac{5}{8}, \frac{6}{8}\right) \in\left\{(x, y) \mid x \geq \frac{1}{2}, y \geq \frac{2}{3}, 2 x+y \leq 2\right\}$. Let

$$
\begin{aligned}
& i_{(\bar{x}, \bar{y})}=(2,2, \ldots), \\
& j_{(\bar{x}, \bar{y})}=(\underbrace{2,2,2,2,2,1,1,1} \underbrace{2,2,2,2,2,1,1,1}, \ldots) .
\end{aligned}
$$


Then, $\left(\gamma_{1}\left(i_{(\bar{x}, \bar{y})}, j_{(\bar{x}, \bar{y})}\right), \gamma_{2}\left(i_{(\bar{x}, \bar{y})}, j_{(\bar{x}, \bar{y})}\right)\right)=(\bar{x}, \bar{y})=\left(\frac{5}{8}, \frac{6}{8}\right)$. However, $\left(i_{(\bar{x}, \bar{y})}, j_{(\bar{x}, \bar{y})}\right)$ is not a Nash equilibrium: If player 1 uses strategy $i_{(\bar{x}, \bar{y})}$, then player 2 can obtain a limiting average reward of 2 by playing his first action at all stages. Let the strategy combination $\left(\pi^{*}, \sigma^{*}\right)$ be given by

$$
\begin{aligned}
& -\pi^{*}\left(h^{t}\right)= \begin{cases}i_{(x, y)}^{t} & \text { if } h^{t}=\left(\left(i_{(\bar{x}, \bar{y})}^{1}, j_{(\bar{x}, \bar{y})}^{1}\right), \ldots,\left(i_{(\bar{x}, \bar{y})}^{t-1}, j_{(\bar{x}, \bar{y})}^{t-1}\right)\right), \\
\pi_{B}^{*} & \text { otherwise; }\end{cases} \\
& \text { - } \sigma^{*}\left(h^{t}\right)= \begin{cases}j_{(x, y)}^{t} & \text { if } h^{t}=\left(\left(i_{(\bar{x}, \bar{y})}^{1}, j_{(\bar{x}, \bar{y})}^{1}\right), \ldots,\left(i_{(\bar{x}, \bar{y})}^{t-1}, j_{(\bar{x}, \bar{y})}^{t-1}\right)\right), \\
\sigma_{A}^{*} & \text { otherwise. }\end{cases}
\end{aligned}
$$

Then, also $\left(\gamma_{1}\left(\pi^{*}, \sigma^{*}\right), \gamma_{2}\left(\pi^{*}, \sigma^{*}\right)\right) \equiv(\bar{x}, \bar{y})=\left(\frac{5}{8}, \frac{6}{8}\right)$. The so-called 'threats' incorporated in the strategy combination $\left(\pi^{*}, \sigma^{*}\right)$ are formulated in the 'otherwise' parts. If player 1 unilaterally deviates by using action 1 at any stage, then he gets an expected stage payoff of $\frac{1}{2} \leq \frac{5}{8}=\bar{x}$ from then on, as player 2 uses $\left(\frac{1}{2}, \frac{1}{2}\right)$ at all further stages. Similarly, if player 2 unilaterally deviates, he gets an expected stage payoff of $\frac{2}{3} \leq \frac{6}{8}=\bar{y}$ at all further stages.

So $\left(\pi^{*}, \sigma^{*}\right)$ is a Nash equilibrium of $(A, B, \infty, \infty)$.

Now, we focus on $\left(r_{1}, r_{2}\right)$-restricted games $\left(A, B, r_{1}, r_{2}\right)$. We introduce several notations in order to build infinite sequences of pure actions.

Definition 4.17 $A$ block for player 1 , denoted by $b_{1}=\left(i_{1}, i_{2}, \ldots, i_{s}\right)$ is a sequence satisfying $\left\{i_{1}, i_{2}, \ldots, i_{s}\right\} \subseteq I$.

A block for player 2 is defined similarly with respect, to his action set $J$. We call a block consisting of a sequence of $s$ actions, an $s$-block. By $(b, b, \ldots)$, we mean the infinite sequence of actions obtained by an infinite repetition of the block $b$. Let $b^{\prime}$ be an $s^{\prime}$-block, i.e., a block of length $s^{\prime}$, and let $c$ be a natural number, then if we write $\left(b, c \cdot b^{\prime}\right)$ for instance, we mean the $\left(s+c \cdot s^{\prime}\right)$-block obtained by taking the $s$-block $b$ first and then taking $c$ repetitions of the block $b^{\prime}$. For $b=(1,2,3), b^{\prime}=(4,4,2,1)$, and $c=2$ we get $\left(b, 2 b^{\prime}\right)=(1,2,3,4,4,2,1,4,4,2,1)$.

Definition 4.18 An s-block $b=\left(i_{1}, i_{2}, \ldots, i_{s}\right)$ for player 1 is admissible if each action in $\left\{i_{1}, i_{2}, \ldots, i_{s}\right\}$ occurs at least once every $r_{1}$ consecutive stages of $(b, b, \ldots)$.

An admissible block for player 2 is defined similarly with respect to restriction $r_{2}$. In general, admissibility of $b^{\prime}$ and $b^{\prime \prime}$ does not imply admissibility of $\left(b^{\prime}, b^{\prime \prime}\right)$. 
Definition 4.19 Let $p \in\{1,2, \ldots, r\}$ with $r=\min \left\{r_{1}, r_{2}\right\}$. Furthermore, let $S=\left\{\left(a_{i_{1}}, b_{j_{1}}\right),\left(a_{i_{2}}, b_{j_{2}}\right), \ldots,\left(a_{i_{p}}, b_{j_{p}}\right)\right\}$ consist of $p$ different entries of $(A, B)$. Then, the sets $I(S)$ and $J(S)$ are given by

$$
\begin{aligned}
& I(S)=\left\{i_{1}, \ldots, i_{p}\right\}, \\
& J(S)=\left\{j_{1}, \ldots, j_{p}\right\} .
\end{aligned}
$$

Furthermore, for $w \in\{1,2, \ldots, p\}$ the $r$-blocks $b_{1}^{w}, b_{2}^{w}$ are given by

$$
\begin{array}{r}
b_{1}^{w}=\left\{\begin{array}{ll}
\left(i_{1}, i_{2}, \ldots, i_{p-1}, i_{p}\right) & \text { if } r=p \\
\left(i_{1}, i_{2}, \ldots, i_{p-1}, i_{p}, i_{w}, \ldots, i_{w}\right) & \text { if } r>p
\end{array},\right. \\
b_{2}^{w}=\left\{\begin{array}{ll}
\left(j_{1}, j_{2}, \ldots, j_{p-1}, j_{p}\right) & \text { if } r=p \\
\left(j_{1}, j_{2}, \ldots, j_{p-1}, j_{p}, j_{w}, \ldots, j_{w}\right) & \text { if } r>p
\end{array} .\right.
\end{array}
$$

Hence, in these $r$-blocks player 1 first plays the sequence $\left(i_{1}, \ldots, i_{p}\right)$, and player 2 first plays the sequence $\left(j_{1}, \ldots, j_{p}\right)$. Then player 1 plays the action $i_{w}$, and player 2 plays the action $j_{w}$, precisely $r-p$ times in succession. Note that $b_{1}^{w}$ is admissible for player 1 and $b_{2}^{w}$ is admissible for player 2. For instance, a sequence

$$
\left(b_{1}^{w}, b_{2}^{w}\right)=\left(\left(i_{1}, j_{1}\right), \ldots,\left(i_{p}, j_{p}\right),\left(i_{w}, j_{w}\right), \ldots,\left(i_{w}, j_{w}\right)\right)
$$

yields average stage payoffs of $\left(X_{w}, Y_{w}\right)$ where

$$
\begin{aligned}
& X_{w}=\frac{r-p}{r} a_{i_{w} j_{w}}+\sum_{w^{\prime}=1}^{p} \frac{a_{i_{w^{\prime}}, j_{w^{\prime}}}}{r}, \\
& Y_{w}=\frac{r-p}{r} b_{i_{w}, j_{w}}+\sum_{w^{\prime}=1}^{p} \frac{b_{i_{w^{\prime}} j_{w^{\prime}}}}{r} .
\end{aligned}
$$

With these notations we formulate the following intermediate result. Now, let $F R\left(A, B, r_{1}, r_{2}\right)$ denote the set of feasible rewards in the game $\left(A, B, r_{1}, r_{2}\right)$.

Lemma 4.20 Conv $\left(\left\{\left(X_{w}, Y_{w}\right) \mid w \in\{1,2, \ldots, p\}\right\}\right) \subseteq F R\left(A, B, r_{1}, r_{2}\right)$ for any $p \leq r$. 
Proof. Take $(a, b)=\sum_{w \in I^{p}} \lambda_{w}\left(X_{w}, Y_{w}\right)$ satisfying $\lambda_{w} \geq 0$ for all $w \in$ $\{1,2, \ldots, p\}$ and $\sum_{w=1}^{p} \lambda_{w}=1$. For $s=1,2, \ldots$, let $\left(a_{s}, b_{s}\right)=\frac{1}{s} \sum_{t=1}^{s}\left(a_{i^{t}, j^{t}}, b_{i^{t}, j^{t}}\right)$ and let $\mu_{w}^{s}, w \in\{1,2, \ldots, p\}$, be such that

$$
\left(a_{s}, b_{s}\right)=\sum_{w=1}^{p} \mu_{w}^{s}\left(X_{w}, Y_{w}\right),
$$

$\sum_{w=1}^{p} \mu_{w}^{s}=1$ and $\mu_{w}^{s} \geq 0$ for all $w \in\{1,2, \ldots, p\}$. We will now construct a sequence of action pairs $\left(\left(i^{1}, j^{1}\right),\left(i^{2}, j^{2}\right), \ldots\right)$ such that

$$
\lim _{s \rightarrow \infty}\left(a_{s}, b_{s}\right)=\lim _{s \rightarrow \infty} \frac{1}{s} \sum_{t=1}^{s}\left(a_{i t}, j^{t}, b_{i^{t}} j^{t}\right)=(a, b),
$$

and

$$
\lim _{t \rightarrow \infty} \mu_{w}^{t}=\lambda_{w} \text { for all } w \in\{1,2, \ldots, p\} .
$$

Choose $\left(\left(i^{1}, j^{1}\right), \ldots,\left(i^{p}, j^{p}\right)\right)=\left(\left(i_{1}, j_{1}\right), \ldots,\left(i_{p}, j_{p}\right)\right)$.

Define for all $k \in\{0,1,2, \ldots\}:\left(\left(i^{p+1+k r}, j^{p+1+k r}\right), \ldots,\left(i^{p+(k+1) r}, j^{p+(k+1) r}\right)\right)$, recursively as follows:

Let $\tilde{w} \in\{1,2, \ldots, p\}$ satisfy:

$$
\left(\mu_{\tilde{w}}^{p+k r}-\lambda_{\tilde{w}}\right)=\min _{w^{\prime} \in I^{p}}\left(\mu_{w^{\prime}}^{p+k r}-\lambda_{w^{\prime}}\right),
$$

and let $b_{1}^{\tilde{w}}, b_{2}^{\tilde{w}}$ be the corresponding $r$-blocks of both players as given in Definition 4.19. Choose:

$$
\left(\left(i^{p+1+k r}, j^{p+1+k r}\right), \ldots,\left(i^{p+(k+1) r}, j^{p+(k+1) r}\right)\right)=\left(b_{1}^{\tilde{w}}, b_{2}^{\tilde{w}}\right) .
$$

So, at every stage $t=p+k r$ for $k \in\{0,1,2, \ldots\}$ an $r$-block is chosen for each player which is to be played from stage $p+k r+1$ until stage $p+(k+1) r$. Then, $\lim _{l \rightarrow \infty} \mu_{w}^{t}=\lim _{k \rightarrow \infty} \mu_{w}^{p+k r}=\lambda_{w}$ for all $w \in I^{p}$. Furthermore, $\lim _{t \rightarrow \infty}\left(a_{t}, b_{t}\right)=\lim _{t \rightarrow \infty} \sum_{w=1}^{p} \mu_{w}^{t}\left(X_{w}, Y_{w}\right)=\lim _{k \rightarrow \infty} \mu_{w}^{p+k r}\left(X_{w}, Y_{w}\right)=$ $\sum_{w=1}^{p} \lambda_{w}\left(X_{w}, Y_{w}\right)=(a, b)$.

With the above we define $(\pi, \sigma)$ by

$$
\left(\pi\left(h^{t}\right), \sigma\left(h^{t}\right)\right)=\left(i^{t}, j^{t}\right) \text { for all } t \in\{1,2, \ldots\} .
$$

Hence, $\left(\gamma_{1}(\pi, \sigma), \gamma_{2}(\pi, \sigma)\right)=(a, b)$. 


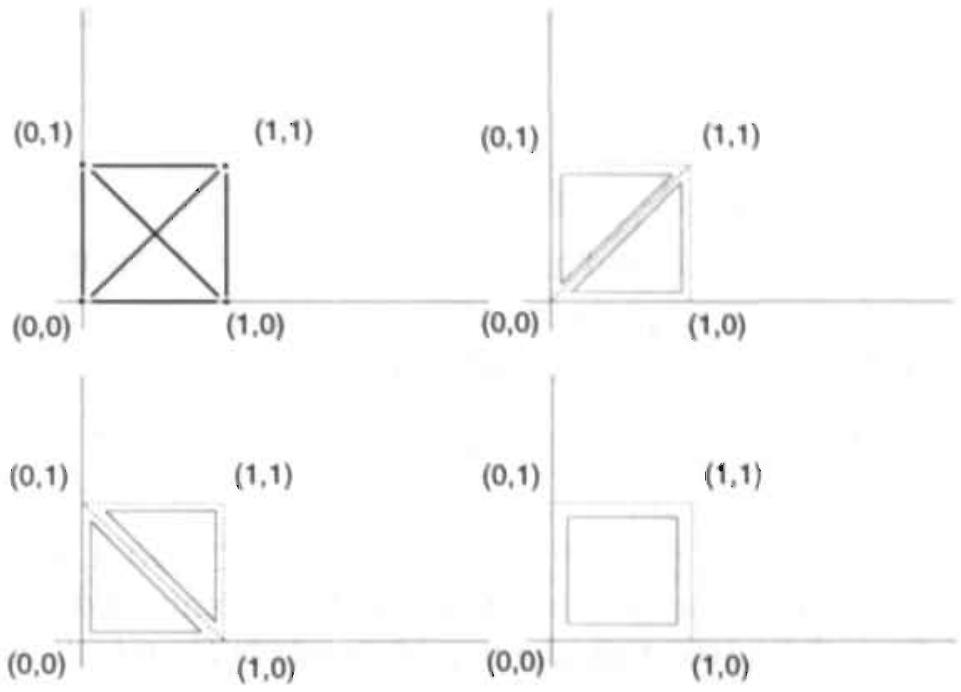

Figure 4.1: Sketch of sets of feasible rewards in Example 4.5.

The similarity of the proof of Lemma, 4.20 to the proof of Lemma 4.15 lies in the idea that in the construction of the infinite sequence of actions in the proof of Lemma 4.15, a decision is made for every stage. In the proof of Lemma 4.20 such a decision is made only once in every $r$ stages, and an $r$-block is chosen for the next $r$ stages.

Example 4.5. Consider $\left(A, B, r_{1}, r_{2}\right)$ with $r_{1}, r_{2} \geq 4$, satisfying

$$
(A, B)=\left[\begin{array}{ll}
0,0 & 0,1 \\
1,0 & 1,1
\end{array}\right] \text {. }
$$

Take: $i_{1}=1$ and $j_{1}=2$, then:

$$
\left(X_{1}, Y_{1}\right)=(0,1) .
$$

Hence, $\{(0,1)\} \subset F R\left(A, B, r_{1}, r_{2}\right)$.

Furthermore, take $i_{1}=i_{2}=1, j_{1}=2, j_{2}=1$, then

$$
\left(X_{1}, Y_{1}\right)=\left(0, \frac{r-1}{r}\right),\left(X_{2}, Y_{2}\right)=\left(0, \frac{1}{r}\right) \text {. }
$$


Then, conv $\left(\left\{\left(0, \frac{r-1}{r}\right),\left(0, \frac{1}{r}\right)\right\}\right) \subset F R\left(A, B, r_{1}, r_{2}\right)$.

Similarly, take $i_{1}=i_{2}=1, i_{3}=2$, and $j_{1}=2, j_{2}=j_{3}=1$, then

$$
\left(X_{1}, Y_{1}\right)=\left(\frac{1}{r}, \frac{r-2}{r}\right),\left(X_{2}, Y_{2}\right)=\left(\frac{1}{r}, \frac{1}{r}\right),\left(X_{3}, Y_{3}\right)=\left(\frac{r-2}{r}, \frac{1}{r}\right) .
$$

Then, conv $\left(\left\{\left(\frac{1}{r}, \frac{r-1}{r}\right),\left(\frac{1}{r}, \frac{1}{r}\right),\left(\frac{r-2}{r}, \frac{1}{r}\right)\right\}\right) \subset F R\left(A, B, r_{1}, r_{2}\right)$.

Take $i_{1}=i_{2}=1, i_{3}=i_{4}=2$, and $j_{1}=j_{4}=2, j_{2}=j_{3}=1$, then

$$
\begin{aligned}
& \left(X_{1}, Y_{1}\right)=\left(\frac{2}{r}, \frac{r-2}{r}\right),\left(X_{2}, Y_{2}\right)=\left(\frac{2}{r}, \frac{2}{r}\right), \\
& \left(X_{3}, Y_{3}\right)=\left(\frac{r-2}{r}, \frac{2}{r}\right),\left(X_{4}, Y_{4}\right)=\left(\frac{r-2}{r}, \frac{r-2}{r}\right) .
\end{aligned}
$$

Then, conv $\left(\left\{\left(\frac{2}{r}, \frac{r-2}{r}\right),\left(\frac{2}{r}, \frac{2}{r}\right),\left(\frac{r-2}{r}, \frac{2}{r}\right),\left(\frac{r-2}{r}, \frac{r-2}{r}\right)\right\}\right) \subset F R\left(A, B, r_{1}, r_{2}\right)$. See Figure 4.1. In the upper left hand sketch, four points and six line segments are indicated, representing the feasible rewards for sets $S$ with $p=1$ and $p=2$ respectively. In the upper right hand and lower left hand sketches, the four triangles indicate the sets of feasible rewards for sets $S$ with $p=3$. In the lower right hand, the square indicates the set of feasible rewards for $p=4(S=\{(0,0),(1,0),(0,1),(1,1)\})$.

Let $F R_{S}\left(A, B, r_{1}, r_{2}\right)=\operatorname{conv}\left\{\left(X_{w}, Y_{w}\right) \mid\left(a_{i_{w}, j_{w}}, b_{i_{w}, j_{w}}\right) \in S\right\}$ for given $S$. The actions in the sets $I(S), J(S)$ are to be kept available in order to obtain limiting average rewards in $F R_{S}\left(A, B, r_{1}, r_{2}\right)$. Let $\pi_{S}, \sigma_{S}$ be strategies for players 1,2 restricted to the sets $I(S)$ and $J(S)$, respectively. Let $\underline{v}_{1}^{S}=$ $\inf _{\sigma_{S}} \sup _{\pi} \gamma_{1}\left(\pi, \sigma_{S}\right)$, and $\underline{v}_{2}^{S}=\inf _{\pi_{S}} \sup _{\sigma} \gamma_{2}\left(\pi_{S}, \sigma\right)$. These amounts $\underline{v}_{1}^{S}, \underline{v}_{2}^{S}$ may be interpreted as follows. If player 2 has all actions in $J(S)$ available, he can keep player 1's limiting average reward at $\underline{v}_{1}^{S}$. Similarly, provided player 1 has all actions in $I(S)$ available, he can keep player 2 at $\underline{v}_{2}^{S}$.

Now, for $\varepsilon>0$, let $\underline{\pi}_{S}^{\varepsilon}$ and $\underline{\sigma}_{S}^{\varepsilon}$ satisfy $\gamma_{2}\left(\underline{\pi}_{S}^{\varepsilon}, \sigma\right) \leq \underline{v}_{2}^{S}+\varepsilon$ for all $\sigma$, and $\gamma_{1}\left(\pi, \underline{\sigma}_{S}^{\varepsilon}\right) \leq \underline{v}_{1}^{S}+\varepsilon$ for all $\pi$. Furthermore, let the set of individually rational rewards in the game $\left(A, B, r_{1}, r_{2}\right)$ with respect to $S$ be given by

$$
\operatorname{IRR}_{S}\left(A, B, r_{1}, r_{2}\right)=\left\{(x, y) \in \Re \times \Re \mid x \geq \underline{v}_{1}^{S}, y \geq \underline{v}_{2}^{S}\right\} .
$$

Let

$$
N E R_{S}^{\epsilon}\left(A, B, r_{1}, r_{2}\right)
$$

denote the set of Nash $\varepsilon$-equilibrium rewards in $\left(A, B, r_{1}, r_{2}\right)$ with respect to $S$. Then, we can state the following. 
Proposition 4.21 For $\left(A, B, r_{1}, r_{2}\right)$, and non-empty set $S$ as given before, it holds that for every

$$
(a, b) \in\left(F R_{S}\left(A, B, r_{1}, r_{2}\right) \cap I R R_{S}\left(A, B, r_{1}, r_{2}\right)\right)
$$

and for every $\varepsilon>0$ there is a Nash $\varepsilon$-equilibrium $\left(\pi_{\varepsilon}, \sigma_{\varepsilon}\right)$ with $\left|\gamma_{1}\left(\pi_{\varepsilon}, \sigma_{\varepsilon}\right)-a\right| \leq$ $\varepsilon$ and $\left|\gamma_{2}\left(\pi_{\varepsilon}, \sigma_{\varepsilon}\right)-b\right| \leq \varepsilon$.

Proof. Let $(a, b) \in F R_{S}\left(A, B, r_{1}, r_{2}\right) \cap I R R_{S}\left(A, B, r_{1}, r_{2}\right)$. In the proof of Lemma 4.20, it was shown that a pair of infinite sequences of pure actions, say,

$$
\begin{aligned}
& i^{\infty}=\left(i^{1}, i^{2}, i^{3} \ldots\right), \\
& j^{\infty}=\left(j^{1}, j^{2}, j^{3} \ldots\right),
\end{aligned}
$$

may be constructed satisfying $\left(a_{i^{t}, j^{t}}, b_{i^{t}, j^{t}}\right) \in S$, and all actions in $I(S), J(S)$ remain available, while $\lim _{\tau \rightarrow \infty} \frac{1}{\tau} \sum_{t=1}^{r}\left(a_{i_{t}, j_{t}}, b_{i_{t} j_{t}}\right)=(a, b)$. Furthermore, let $\underline{\pi}_{S}^{\varepsilon}$ and $\underline{\sigma}_{S}^{\varepsilon}$ be given as defined above. Now, let $\left(\pi^{*}, \sigma^{*}\right)$ be given by

$$
\begin{aligned}
& \text { - } \pi^{*}\left(h^{t}\right)= \begin{cases}i^{t} & \text { if } h^{t}=\left(\left(i^{1}, j^{1}\right),\left(i^{2}, j^{2}\right), \ldots,\left(i^{t-1}, j^{t-1}\right)\right) \\
\underline{\pi}_{S}^{\epsilon} & \text { otherwise; }\end{cases} \\
& \text { - } \sigma^{*}\left(h^{t}\right)= \begin{cases}j^{t} & \text { if } h^{t}=\left(\left(i^{1}, j^{1}\right),\left(i^{2}, j^{2}\right), \ldots,\left(i^{t-1}, j^{t-1}\right)\right) \\
\underline{\sigma}_{S}^{\epsilon} & \text { otherwise. }\end{cases}
\end{aligned}
$$

If play develops according to $\left(i^{\infty}, j^{\infty}\right)$, then the limiting average rewards are equal to $(a, b)$. Suppose player 2 uses $\sigma^{*}$ and player 1 deviates at some point in time from $i^{\infty}$, then he will receive an average stage-payoff of at most $\underline{v}_{1}^{S}+\varepsilon \leq a+\varepsilon$ from then on as player 2 will play $\underline{\sigma}_{S}^{\varepsilon}$ from then on. (The case for player 2 is similar.) This implies that $\left(\pi^{*}, \sigma^{*}\right)$ is a Nash $\varepsilon$-equilibrium of $\left(A, B, r_{1}, r_{2}\right)$.

\subsubsection{Existence of a Nash equilibrium for $\left(r_{1}, r_{2}\right)$ with $\mathbf{r}_{1} \leq 2$ or $\mathbf{r}_{2} \leq 2$}

We begin this subsection with a straightforward result for $r_{1}=r_{2}=1$.

Lemma 4.22 The following two statements are equivalent: 
i. $\left(\pi^{*}(1), \sigma^{*}(1)\right)$ is a Nash equilibrium for the game $(A, B)$,

ii. $\left(\pi^{*}, \sigma^{*}\right)$ satisfying $\pi^{*}\left(h^{1}\right)=\pi^{*}(1)$ and $\sigma^{*}\left(h^{1}\right)=\sigma^{*}(1)$, is a Nash equilibrium in $(A, B, 1,1)$.

Hence, each game $(A, B, 1,1)$ possesses a Nash equilibrium, and the first stage action-combination is exactly a one-shot bi-matrix game Nash equilibrium. We introduce the following notations: $J(i)=\left\{j \in J \mid b_{i, j}=\max _{k \in J} b_{i, k}\right\}$, and $j(i) \in\left\{j \in J(i) \mid a_{i, j}=\min _{k \in J(i)} a_{i, k}\right\}$. Let action $i^{*}$ satisfy $a_{i^{*}, j\left(i^{*}\right)}=$ $\max _{i \in I} a_{i, j(i)}$. Then, we can state the following two results.

Proposition 4.23 The following strategy combination is a Nash equilibrium for $(A, B, 1, r)$ for all $r \geq 2$ :

- For player 1: Play action $i^{*}$ at all stages;

- For player 2: Play action 1 at stage 1 . For all stages $t \geq 2$, play any action yielding the highest stage-payoff for player 2 against player 1's remaining action.

The case $r_{1} \geq 2, r_{2}=1$ is analogous.

For $r \geq 3$, we show that a Nash equilibrium for $(A, B, 2, r)$ yields limiting average rewards equal to $\left(a_{i^{*}, j\left(i^{*}\right)}, b_{i^{*}, j\left(i^{*}\right)}\right)$.

Proposition 4.24 The following strategy combination is a Nash equilibrium of $(A, B, 2, r)$ with $r \geq 3$ :

- For player 1: Play action $i^{*}$ at all stages;

- For player 2: At stage 1 select action 1, at stage 2 select action $j\left(i^{1}\right)$, at stage 3 select $j\left(i^{2}\right)$, followed by:

a. As long as player 1 has two actions available, play $j\left(i^{1}\right), j\left(i^{2}\right)$ alternately, starting with $j\left(i^{2}\right)$ at stage 4 ;

b. If player 1 has only action $i^{s}, s \in\{1,2\}$ available at any stage, choose $j\left(i^{s}\right)$ from then on. 
Proof. This strategy-combination yields $\left(a_{i^{*}} j_{\left(i^{*}\right)}, b_{i^{*}, j\left(i^{*}\right)}\right)$ as limiting average rewards. Let player 1 use $i^{*}$ at all stages, then player 2 can get a stage payoff of at most $b_{i{ }^{*} j\left(i^{*}\right)}$ at each stage. Hence, his limiting average award is at most $b_{i^{*}, j\left(i^{*}\right)}$. Let player 2 use the strategy given, then at stage 4 , player 1 either has one action available, say $i^{\prime}$, or two actions, say $i_{1}, i_{2}$. In the first case player 1 receives a stage payoff of $a_{i^{\prime}, j\left(i^{\prime}\right)}$ from then on. Hence, his limiting average reward is $a_{i^{\prime} j\left(i^{\prime}\right)} \leq a_{i \cdot j\left(i^{*}\right)}$. In the second case, player 1 receives a stage payoff of at most $\max \left\{a_{i_{1} j\left(i_{1}\right)}, a_{i_{2} j\left(i_{2}\right)}\right\} \leq a_{i^{*}, j\left(i^{*}\right)}$.

Again, the case $r_{2}=2, r_{1} \geq 3$ is analogous.

For the purpose of showing existence of a Nash equilibrium of arbitrary games $(A, B, 2,2)$, we derive several intermediate results for the case where each player has two actions at the start of play. The following is straightforward and well-known.

Lemma 4.25 The bi-matrix game $(A, B)$ does not have a pure Nash equilibrium if and only if either one of the following lists of inequalities hold:

$$
\begin{aligned}
& \text { - } a_{1,1}<a_{2,1}, b_{2,1}<b_{2,2}, a_{2,2}<a_{1,2}, b_{1,2}<b_{1,1} \\
& \text { - } a_{1,1}>a_{2,1}, b_{2,1}>b_{2,2}, a_{2,2}>a_{1,2}, b_{1,2}>b_{1,1} \text {. }
\end{aligned}
$$

We call a diagonal of a $(2 \times 2)$-matrix good if the sum of its elements is strictly higher than the sum of the elements of the other diagonal. The other diagonal will be called the bad diagonal. If we use the phrase 'the good diagonal for player 1' for instance, we mean the good diagonal in payoff matrix $A$. For the first series of inequalities of the lemma above, the diagonal $((1,2),(2,1))$ is the good. diagonal in $A$, since $a_{1,2}+a_{2,1}>a_{1,1}+a_{2,2}$. The diagonal $((1,1),(2,2))$ is the good one in $B$ since $b_{1,1}+b_{2,2}>b_{1,2}+b_{2,1}$. This leads to the following.

Corollary 4.26 If a $(2 \times 2)$-bi-matrix game $(A, B)$ does not possess a pure. Nash equilibrium, then both $A$ and $B$ have a good diagonal and a bad diagonal. Moreover, the good diagonal in $A$ corresponds with the bad one in $B$, and vice versa.

Proposition 4.27 For any $(2 \times 2)$-game $(A, B, 2,2)$ there exists a Nash equilibrium. 
Proof. In view of Lemma 4.11, we only prove existence of Nash equilibrium for games $(A, B, 2,2)$ where $(A, B)$ does not have a pure Nash equilibrium. Define $\pi^{*}$ by:

- Play $\left(\frac{1}{2}, \frac{1}{2}\right)$ at the first stage, and next:

- If the good diagonal of $A$ is reached at the first stage of play, then proceed with:

a. Keep both actions alive as long as player 2 has two actions;

b. If player 2 has only one action left, then choose an action yielding the highest one-stage payoff, at all further stages.

- If the bad diagonal of $A$ is reached at the first stage of play, then choose the action corresponding with the highest bad diagonal payoff from stage 2 onwards.

Define $\sigma^{*}$ similarly with respect to $B$. Then $\left(\pi^{*}, \sigma^{*}\right)$ is a Nash equilibrium. This may be seen as follows. Without loss of generality we may assume that $((1,1),(2,2))$ is the good diagonal for player 1 , hence the good diagonal of player 2 is $((1,2),(2,1))$ by Lemma 4.25 . We may furthermore assume without loss of generality that $a_{2,1}>a_{1,2}$ and $b_{1,1}>b_{2,2}$. Suppose player 2 uses $\sigma^{*}$. Then with probability $\frac{1}{2}$ player 2 's bad diagonal is reached at the first stage of play. In that case player 2 plays action 1 from then on. Hence, player 1 maximizes his stage-payoff from then on by choosing action 1 as well. With probability $\frac{1}{2}$ player 2's good diagonal is reached at the first stage. Player 1 then has essentially three options: Keep both actions available, drop action 2, or drop action 1. The first option yields an average stage payoff of $\frac{1}{2}\left(a_{1,2}+a_{2,1}\right)$ as both players will switch forever, the second one yields $a_{1,2}$, and the third one yields $a_{2,1}$. Hence, player 1 maximizes his average stage payoff by choosing action 2 from then on. Since player 1 cannot influence which diagonal will be reached at the first stage of play, he may choose $\left(\frac{1}{2}, \frac{1}{2}\right)$. Therefore, $\pi^{*}$ maximizes player 1's limiting average reward when player 2 uses $\sigma^{*}$. Similarly, if player 1 uses $\pi^{*}$, then $\sigma^{*}$ maximizes player 2's limiting average reward.

We now show existence of a Nash equilibrium for general games $(A, B, 2,2)$. After two stages of play both players lose all actions except the ones selected 
at the first two stages. So the remaining game which the players face at stage 3 , will generally have size $2 \times 2$. We do not consider remaining games of smaller size because the activity of choosing the same action $x$ at stages 1 and 2 , is weakly dominated by the activity of choosing $x$ at the first stage and any action $y \neq x$ at stage 2 .

Suppose entry $(1,1)$ has been realized at stage 1 , and suppose $(i, j) \neq$ $(1,1)$ is chosen at stage 2 , so $h^{3}=((1,1),(i, j))$. Then, the remaining game after stage 2 is

$$
\left(A_{h^{3}}, B_{h^{3}}\right)=\left[\begin{array}{cc}
a_{1,1}, b_{1,1} & a_{1, j}, b_{1, j} \\
a_{i, 1}, b_{i, 1} & a_{i, j}, b_{i, j}
\end{array}\right] .
$$

Then, one of three cases (cf. Corollary 4.26) occurs:

1. The game $\left(A_{h^{3}}, B_{h^{3}}\right)$ possesses a pure Nash equilibrium, say, $\left(i^{*}, j^{*}\right)$ with $i^{*} \in\{1, i\}, j^{*} \in\{1, j\}$;

2. The diagonal $((1,1),(i, j))$ is player 1's good diagonal, and at the same time it is player 2's bad diagonal;

3. The diagonal $((1, j),(i, 1))$ is player 2's good diagonal, and at the same time it is player 1's bad diagonal.

In Case 1 , the players may use $\left(i^{*}, j^{*}\right)$ at all stages $t \geq 3$, and the average payoffs will be $\left(a_{i^{*}, j^{*}}, b_{i^{*}, j^{*}}\right)$. Suppose player 1 indeed uses action $i^{*}$ at all stages $t \geq 3$, then player 2 maximizes his stage payoff by using action $j^{*}$ at all further stages. Similarly, suppose player 2 uses $j^{*}$ at all further stages then player 1 maximizes his stage-payoffs by using action $i^{*}$.

If Case 2 occurs, assume without loss of generality that $b_{1,1} \geq b_{i, j}$. Player 1's good diagonal is reached, and player 1 may proceed from stage 3 (cf. the proof of Proposition 4.27):

- Keep both actions alive as long as player 2 has two actions available;

- If player 2 loses an action choose the action yielding the highest stage payoff from then on.

Player 2 can proceed (cf. the proof of Proposition 4.27) with:

- Choose the first action from stage 3 on. Then, in that case the average stage-payoffs will be $\left(a_{1,1}, b_{1,1}\right)$. 
By the arguments used in the proof of Proposition 4.27, neither player can unilaterally improve his average stage-payoff from then on.

In Case 3, assume without loss of generality that $a_{1,1} \leq a_{i, j}$. Players 1 and 2 may proceed from stage 3 on as described in the proof of Proposition 4.27 as well. Hence, the average stage-payoffs will be $\left(a_{i, j}, b_{i, j}\right)$ from then on. Neither player can unilaterally improve his average stage-payoff at any further stage of play.

Let us introduce several notations with respect to $h^{3}=((1,1),(i, j))$ :

$$
\left(w_{h^{3}}^{1}, w_{h^{3}}^{2}\right)= \begin{cases}\left(a_{i^{*}, j^{*}}, b_{i^{*}, j^{*}}\right) & \text { in Case 1, } \\ \left(a_{1,1}, b_{1,1}\right) & \text { in Case 2, } \\ \left(a_{i, j}, b_{i, j}\right) & \text { in Case 3 }\end{cases}
$$

$$
\begin{aligned}
& \pi_{h^{3}}=\left\{\begin{array}{ll}
\left(i^{*}, i^{*}, \ldots\right) & \\
(1, i, 1, i, \ldots) & \text { as long as player 2 has actions 1,j } \\
1 & \text { once player 2 has only action 1 left } \\
i & \text { once player 2 has only action } j \text { left }
\end{array}\right\} \text { in Case 2, } \\
& (i, i, \ldots) \quad \text { in Case 3, } \\
& \sigma_{h^{3}}^{*}=\left\{\begin{array}{ll}
\left(j^{*}, j^{*}, \ldots\right) & \text { in Case 1, } \\
(1,1, \ldots) & \text { in Case 2, } \\
(1, j, 1, j, \ldots) & \text { as long as player 1 has actions 1,i } \\
j & \text { once player 1 has only action 1 left } \\
1 & \text { once player 1 has only action } i \text { left }
\end{array}\right\} \text { in Case 3. }
\end{aligned}
$$

The pairs $\left(w_{h^{3}}^{1}, w_{h^{3}}^{2}\right)$ and $\left(\pi_{h^{3}}^{*}, \sigma_{h^{3}}^{*}\right)$ may be interpreted as follows. If $h^{3 i}=$ $((1,1),(i, j))$, then the strategy-combination $\left(\pi_{h^{3}}^{*}, \sigma_{h^{3}}^{*}\right)$ yields average stagepayoffs of $\left(w_{h^{3}}^{1}, w_{h^{3}}^{2}\right)$ from stage 3 on, and neither player can achieve a higher average stage-payoff by deviating unilaterally from $\left(\pi_{h^{3}}^{*}, \sigma_{h^{3}}^{*}\right)$.

The same may be done for all $h^{3}=((1,1),(i, j))$ satisfying $i \neq 1, j \neq 1$. Let, for $h^{2}=((1,1)) W_{h^{2}}^{1}=\left[w_{((1,1),(i, j))}^{1}\right]_{i \neq 1, j \neq 1}$ and $W_{h^{2}}^{2}=\left[w_{((1,1),(i, j))}^{1}\right]_{i \neq 1, j \neq 1}$. Determine for $\left(W_{(1,1)}^{1}, W_{(1,1)}^{2}\right)$ a Nash equilibrium $\left(\pi_{(1,1)}^{*}, \sigma_{(1,1)}^{*}\right)$. Let $\left(a_{(1,1)}^{*}, b_{(1,1)}^{*}\right)$ be the expected Nash equilibrium rewards, to be interpreted as follows. If entry $(1,1)$ is reached at the first stage of the game, then $\left(a_{(1,1)}^{*}, b_{(1,1)}^{*}\right)$ are the expected average rewards from stage 2 on, to be achieved by playing $\left(\pi_{(1,1)}^{*}, \sigma_{(1,1)}^{*}\right)$ at stage 2 , followed by $\left(\pi_{((1,1),(i, j))}^{*}, \sigma_{((1,1),(i, j))}^{*}\right)$ if entry $(i, j)$ with $i \neq 1, j \neq 1$, is reached at stage 2 . Neither player can improve his average stage-payoff from stage 2 on by deviating unilaterally from this strategy 
given that entry $(1,1)$ is reached at stage 1 .

Construct $A^{*}=\left[a_{(i, j)}^{*}\right]$ and $B^{*}=\left[b_{(i, j)}^{*}\right]$ with $a_{(i, j)}^{*}$ and $b_{(i, j)}^{*}$ determined conditional on entry $(i, j)$ being reached at the first stage in a manner similar to the one just described in detail for entry $(1,1)$. Determine for $\left(A^{*}, B^{*}\right)$ a Nash equilibrium $\left(\pi^{*}(1), \sigma^{*}(1)\right)$ and let the corresponding expected payoffs be equal to $\left(v_{1}^{*}, v_{2}^{*}\right)$. Let $\left(\pi^{*}, \sigma^{*}\right)$ be given by:

- $\left(\pi^{*}\left(h^{1}\right), \sigma^{*}\left(h^{1}\right)\right)=\left(\pi^{*}(1), \sigma^{*}(1)\right)$,

- if $h^{2}=((i, j))$, then let $\left(\pi^{*}\left(h^{2}\right), \sigma^{*}\left(h^{2}\right)\right)=\left(\pi_{h^{2}}^{*}, \sigma_{h^{2}}^{*}\right)=\left(\pi_{(i, j)}^{*}, \sigma_{(i, j)}^{*}\right)$,

- if $h^{3}=((i, j),(k, l))$, then $\left(\left(\pi^{*}\left(h^{3}\right), \pi^{*}\left(h^{4}\right), \ldots\right)=\pi_{h^{3}}^{*}=\pi_{((i, j),(k, l))}^{*}\right.$ and $\left(\left(\sigma^{*}\left(h^{3}\right), \sigma^{*}\left(h^{4}\right), \ldots\right)=\sigma_{h^{3}}^{*}=\sigma_{((i, j),(k, l))}^{*}\right.$

By backward induction it follows that $\left(\pi^{*}, \sigma^{*}\right)$ is a Nash equilibrium of $(A, B, 2,2)$ and $\left(\gamma_{1}\left(\pi^{*}, \sigma^{*}\right), \gamma_{2}\left(\pi^{*}, \sigma^{*}\right)\right)=\left(v_{1}^{*}, v_{2}^{*}\right)$.

Based on the preceding we formulate the following algorithm to calculate a Nash equilibrium of a $(2,2)$-restricted general-sum game.

\section{Algorithm 4.3.}

Step 1. Take $i \in I, j \in J$, examine the bi-matrix games $\left(A_{h^{3}}, B_{h^{3}}\right)$, for $h^{3}=((i, j),(k, l))$ satisfying $k \neq i, l \neq j$. Determine $\left(\pi_{h^{3}}^{*}, \sigma_{h^{3}}^{*}\right)$ and $\left(w_{h^{3}}^{1}, w_{h^{3}}^{2}\right)$ corresponding with $\left(A_{h^{3}}, B_{h^{3}}\right)$ for each $h^{3}=((i, j),(k, l))$ with $k \neq i, l \neq j$, as described.

Step 2. Construct for $h^{2}=((i, j))$ the bi-matrix game $\left(W_{h^{2}}^{1}, W_{h^{2}}^{2}\right)$ with

$$
W_{h^{2}}^{1}=\left[w_{((i, j),(k, l))}^{1}\right]_{k \neq i, l \neq j} \text { and } W_{h^{2}}^{2}=\left[w_{((i, j),(k, l))}^{2}\right]_{k \neq i, l \neq j} .
$$

Determine for $h^{2}=((i, j))$ a Nash equilibrium $\left(\pi_{h^{2}}^{*}, \sigma_{h^{2}}^{*}\right)$ of the bi-matrix game $\left(W_{h^{2}}^{1}, W_{h^{2}}^{2}\right)$ and the corresponding expected payoffs $\left(a_{h^{2}}^{*}, b_{h^{2}}^{*}\right)$.

Step 3. Repeat Steps 1 and 2 for all $h^{2}=((i, j))$ with $i \in I, j \in J$. Let $\left(A^{*}, B^{*}\right)$ be the bimatrix the entry $(i, j)$ of which equals $\left(a_{(i, j)}^{*}, b_{(i, j)}^{*}\right)$.

Step 4. Determine a Nash equilibrium $\left(\pi^{*}(1), \sigma^{*}(1)\right)$ for the bi-matrix game $\left(A^{*}, B^{*}\right)$ and let $\left(v_{1}^{*}, v_{2}^{*}\right)$ be the corresponding equilibrium payoff.

Step 5. Now, a Nash equilibrium for $(A, B, 2,2)$, is given by $\left(\pi^{*}, \sigma^{*}\right)$ with: 
- $\left(\pi^{*}\left(h^{1}\right), \sigma^{*}\left(h^{1}\right)\right)=\left(\pi^{*}(1), \sigma^{*}(1)\right)$,

- $\left(\pi^{*}\left(h^{2}\right), \sigma^{*}\left(h^{2}\right)\right)=\left(\pi_{h^{2}}^{*}, \sigma_{h^{2}}^{*}\right)$,

$\left\{\begin{array}{l}\left(\left(\pi^{*}\left(h^{3}\right), \pi^{*}\left(h^{4}\right), \ldots\right)=\pi_{h^{3}}^{*}\right. \\ \left(\left(\sigma^{*}\left(h^{3}\right), \sigma^{*}\left(h^{4}\right), \ldots\right)=\sigma_{h^{3}}^{*}\right.\end{array}\right.$.

The expected average rewards for $\left(\pi^{*}, \sigma^{*}\right)$ are given by:

$$
\left(\gamma_{1}\left(\pi^{*}, \sigma^{*}\right), \gamma_{2}\left(\pi^{*}, \sigma^{*}\right)\right)=\left(v_{1}^{*}, v_{2}^{*}\right) .
$$

Example 4.6. Consider the game $(A, B, 2,2)$ with

$$
(A, B)=\left[\begin{array}{lll}
2,1 & 2,2 & 1,3 \\
1,4 & 3,0 & 2,4 \\
0,2 & 0,1 & 3,0
\end{array}\right]
$$

We examine all $\left(A_{h^{3}}, B_{h^{3}}\right)$, satisfying $h^{3}=((1,1),(i, j))$, with $i, j \neq 1$.

- For $h^{3}=((1,1),(2,2))$ we have $\left(A_{h^{3}}, B_{h^{3}}\right)=\left[\begin{array}{ll}2,1 & 2,2 \\ 1,4 & 3,0\end{array}\right]$, $\left(w_{h^{3}}^{1}, w_{h^{3}}^{2}\right)=(2,1)$ and

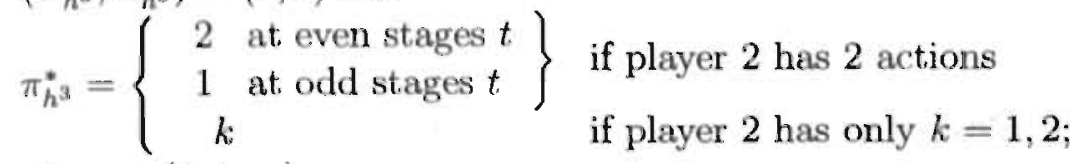
$\sigma_{h^{3}}^{*}=(1,1, \ldots)$.

- For $h^{3}=((1,1),(2,3))$ we have $\left(A_{h^{3}}, B_{h^{3}}\right)=\left[\begin{array}{ll}2,1 & 1,3 \\ 1,4 & 2,4\end{array}\right]$, $\left(w_{h^{3}}^{1}, w_{h^{3}}^{2}\right)=(2,4), \pi_{h^{3}}^{*}=(2,2, \ldots)$, and $\sigma_{h^{3}}^{*}=(3,3, \ldots)$.

- For $h^{3}=((1,1),(3,2))$ we have $\left(A_{h^{3}}, B_{h^{3}}\right)=\left[\begin{array}{ll}2,1 & 2,2 \\ 0,2 & 0,1\end{array}\right]$, $\left(w_{h^{3}}^{1}, w_{h^{3}}^{2}\right)=(2,2), \pi_{h^{3}}^{*}=(1,1, \ldots)$, and $\sigma_{h^{3}}^{*}=(2,2, \ldots)$.

- For $h^{3}=((1,1),(3,3))$ we have $\left(A_{h^{3}}, B_{h^{3}}\right)=\left[\begin{array}{ll}2,1 & 1,3 \\ 0,2 & 3,0\end{array}\right]$, $\left(w_{h^{3}}^{1}, w_{h^{3}}^{2}\right)=(2,1)$, 


$$
\begin{aligned}
& \pi_{h^{3}}^{*}=\left\{\begin{array}{c}
1 \text { at odd stages } t \\
3 \text { at even stages } t
\end{array}\right\} \begin{array}{l}
\text { while player } 2 \text { has } 2 \text { actions, } \\
k
\end{array} \\
& \sigma_{h^{3}}^{*}=(1,1, \ldots) .
\end{aligned}
$$

We obtain the bi-matrix game $\left(W_{(1,1)}^{1}, W_{(1,1)}^{2}\right)$ which is given by

$$
\left(W_{(1,1)}^{1}, W_{(1,1)}^{2}\right)=\left[\begin{array}{ll}
2,1 & 2,4 \\
2,2 & 2,1
\end{array}\right] .
$$

Both $(3,2)$ and $(2,3)$ yield a Nash equilibrium in the game $\left(W_{(1,1)}^{1}, W_{(1,1)}^{2}\right)$. Take $\left(a_{(1,1)}^{*}, b_{(1,1)}^{*}\right)=(2,4)$ and $\left(\pi_{1,1}^{*}, \sigma_{i, 1}^{*}\right)=(2,3)$.

Proceeding in similar manner for all entries, we obtain a bimatrix game

$$
\left(A^{*}, B^{*}\right)=\left[\begin{array}{ccc}
2,4 & 2,2 & 2,4 \\
2,4 & 2, \frac{16}{7} & 2,4 \\
1,3 & 2,4 & 2, \frac{8}{5}
\end{array}\right]
$$

Clearly, $(1,1)$ is a Nash equilibrium in $\left(A^{*}, B^{*}\right)$. Then, a Nash equilibrium for $(A, B, 2,2)$ is $\left(\pi^{*}, \sigma^{*}\right)$ given by:

- $\left(\pi^{*}\left(h^{1}\right), \sigma^{*}\left(h^{1}\right)\right)=(1,1)$;

- $\left(\pi^{*}\left(h^{2}\right), \sigma^{*}\left(h^{2}\right)\right)=(2,3)$;

- $\left(\pi^{*}\left(h^{3}\right), \sigma^{*}\left(h^{3}\right)\right)=\left(\pi_{h^{3}}^{*}, \sigma_{h^{3}}^{*}\right)$ with $\pi_{h^{3}}^{*}=(1,1, \ldots)$ if $h^{3}=((1,2),(2,1))$ or $h^{3}=((1,3)(2,1))$, $\pi_{h^{3}}^{*}=(2,2, \ldots)$ if $h^{3}=((1,1),(2,3)), h^{3}=((1,3),(2,1))$, or $h^{3}=$ $((1,3),(2,2))$.

For all other $h^{3}=((1, k),(2, l)), k, l \in\{1,2,3\}$ :

$\pi_{h^{3}}^{*}=\left\{\begin{array}{c}2 \text { at even stages } t \\ 1 \text { at odd stages } t \\ k\end{array}\right\} \begin{aligned} & \text { while player } 2 \text { has } 2 \text { actions, } \\ & \text { if player } 2 \text { has only } k=1,2,\end{aligned}$

and

$\sigma_{h^{3}}^{*}=(1,1, \ldots)$ if $h^{3}=((1,1),(3,3)),((2,1),(3,3)),((3,1),(1,3))$, or $((3,1),(2,3))$;

$\sigma_{h^{3}}^{*}=(3,3, \ldots)$ if $h^{3}=((1,1),(2,3))$.

For all other $h^{3}=((k, 1),(l, 3)), k, l \in\{1,2,3\}$ :

$\sigma_{h^{3}}^{*}=\left\{\begin{array}{c}3 \text { at even stages } t \\ 1 \text { at odd stages } t \\ 3\end{array}\right\} \begin{aligned} & \text { while player } 1 \text { has } 2 \text { actions, } \\ & \text { otherwise. }\end{aligned}$ 
The expected payoffs are given by $\left(\gamma_{1}\left(\pi^{*}, \sigma^{*}\right), \gamma_{2}\left(\pi^{*}, \sigma^{*}\right)\right)=(2,4)$.

In this example, several pure Nash equilibria exist for $\left(A^{*}, B^{*}\right)$. Generally that need not be true. Similarly, $\left(W_{(i, j)}^{1}, W_{(i, j)}^{2}\right)$ need not have a pure Nash equilibrium. Indeed, in the example above $\left(W_{(2,2)}^{1}, W_{(2,2)}^{2}\right)$ is given by:

$$
\left(W_{2,2}^{1}, W_{2,2}^{2}\right)=\left[\begin{array}{ll}
2,1 & 2,4 \\
1,4 & 3,0
\end{array}\right] \text {. }
$$

This bi-matrix game only has $\left(\pi_{(2,2)}^{*}, \sigma_{(2,2)}^{*}\right)=\left(\left(\frac{4}{7}, \frac{3}{7}\right),\left(\frac{1}{2}, \frac{1}{2}\right)\right)$ as a mixed Nash equilibrium with expected payoffs equal to $\left(2, \frac{16}{7}\right)$.

\subsection{Differential games with changing payoffs}

In a differential game, the players choose actions in continuous time, thereby receiving a flow of payoffs. Such actions are called controls, and they are chosen subject to certain constraints, in particular with respect to a state variable. Such a constraint is called the state equation or transition equation. Differential games are often analyzed by methods provided by optimal control theory, or by dynamic programming (see for instance Starr \& Ho $[1969 \mathrm{a}, \mathrm{b}])$. The models to be presented in the remainder of this section belong to a wide class of games studied in economics in order to analyze, e.g., industrial R\&D (e.g., Reinganum [1982]), joint exploitation of a common exhaustible resource (e.g., Levhari \& Mirman [1980]), or capital accumulation (e.g., Spence [1979]). See also Vega-Redondo [1994].

\subsubsection{An investment problem}

In this subsection we analyze a differential game corresponding to a stylized economic problem of choosing between two ways to invest money. Specifically, we consider a two-player game in continuous time where at each point of time $t \in[0, \infty)$ each player has one (perfectly divisible) unit of money to invest. Each player can divide this one unit between on the one hand a project for which the payoff depends on the investments of both players, and on the other hand a project for which the payoff depends only on own investment. An investment in the first project will, moreover, result in an additional payoff stream, depending on both own investment and the investment of the opponent. This is meant to capture the idea of learning or 
unlearning as explained in the introduction. One may think of increasing or decreasing one's skill/technology ${ }^{1}$ or market share. These additional payoffs constitute state variables.

Let $\alpha(t) \in[0,1]$ and $\beta(t) \in[0,1]$ denote the investment decisions at time $t$ of players 1 and 2 , respectively, in the first project. Let $g(\alpha(t), \beta(t))$ and $h(\alpha(t), \beta(t))$ denote the resulting immediate payoffs at time $t$ for players 1 and 2 , respectively. The function $g$ can be assumed to have obvious properties, like being increasing in $\alpha$ and decreasing in $\beta$. Similarly for $h$. In this basic formulation, however, we do not need such assumptions. We just assume that both functions are continuously differentiable, but that assumption may also be relaxed. As functions of $t$, however, we require that $\alpha$ and $\beta$ have only isolated points of discontinuity, in order to ensure the existence of the integrals below.

The immediate payoffs from investment in the second project at time $t$ are equal to $1-\alpha(t)$ and $1-\beta(t)$, if $\alpha(t)$ and $\beta(t)$ are the investments in the first project, respectively. (Un)learning effects for player 1 are assumed to be captured by a state variable $x$ depending on $\alpha$ as well as on $\beta$ by the state equation $\dot{x}(t)=\alpha(t)-\beta(t)$ (where the dot denotes time derivative). The additional resulting payoff stream for player 1 is given by $x(t) e^{-r t}$, where $r$ may be any real number. Here, $x(t)$ expresses the amount of "learning" relative to the opponent, whereas $e^{-r t}$ describes its long-run effect. Unlearning effects are stressed when $r$ is positive; note that in that case in the long run the term $x(t) e^{-r t}$ practically vanishes, so that only short term effects are interesting. A formulation of the problem where this is avoided, i.e., where also long term effects are interesting, is given in Subsection 4.4.4.

Similarly, (un)learning effects for player 2 are given by a state variable $y$ governed by the state equation $\dot{y}(t)=\beta(t)-\alpha(t)$. The corresponding additional payoff stream is given by $y(t) e^{-s t}$, for some real number $s$. Note that $x(t)+y(t)$ is constant, so one can think of $x(t)$ as the market share of player 1 at time $t$. According to this interpretation, the constants $x_{0}$ and $y_{0}$ in the two maximization problems to follow can be seen as the initial market shares, and it would be natural to choose $x_{0}+y_{0}$ equal to 1 . The case of actual learning would correspond to both initial values being set. equal to 0 .

We can now write down player 1's maximization problem for any given

\footnotetext{
${ }^{1}$ See Cheng $[1984]$ on this topic.
} 
investment plan $\beta(t)(t \in[0, \infty))$ of player 2 and any discount factor $\rho$ :

$$
\begin{array}{cc}
\text { Maximize } & \int_{0}^{\infty} e^{-\rho t}\left[g(\alpha(t), \beta(t))+(1-\alpha(t))+x(t) e^{-r t}\right] d t \\
\text { subject to } & \left\{\begin{array}{c}
\dot{x}(t)=\alpha(t)-\beta(t) \\
x(0)=x_{0}, \alpha(t) \in[0,1] .
\end{array}\right.
\end{array}
$$

Similarly, for player 2 , given investments $\alpha(t)$ of player 1 at each moment: $t \in[0, \infty):$

$$
\begin{array}{ll}
\text { Maximize } & \int_{0}^{\infty} e^{-\rho t}\left[h(\alpha(t), \beta(t))+(1-\beta(t))+y(t) e^{-r t}\right] d t \\
\text { subject to } & \left\{\begin{array}{c}
\dot{y}(t)=\beta(t)-\alpha(t) \\
y(0)=y_{0}, \beta(t) \in[0,1] .
\end{array}\right.
\end{array}
$$

Thus, the players are assumed to maximize discounted streams of payoffs (with common discount factor $\rho$ ), given the investment plans of their opponents. The initial conditions for the state variables $x$ and $y$ are included to make the maximization problems well-defined, but play no role in our analysis.

Observe that, in a seemingly more general but equivalent formulation, the terms $1-\alpha$ and $1-\beta$ in the objective functions could be taken into the functions $g$ and $h$, respectively.

A simultaneous solution of problems (4.1) and (4.2) is a Nash equilibrium for this game. Depending on the nature of the strategies (investment plans) employed, we distinguish between open-loop strategies and closed-loop (feedback) strategies. In the latter case, strategies may depend on the state variables, and the players have the possibility to adapt their action choices while the game is being played. In the former case, a strategy depends only on time and not on the state variables. We will concentrate on open-loop strategies, which are much easier to calculate.

Solving problems (4.1) and (4.2) is a straightforward application of optimal control theory, specifically, of Pontryagin's maximum principle. The Hamiltonian corresponding to problem (4.1) is the function.

$$
H(\alpha, x, t, \lambda)=e^{-\rho t}\left[g(\alpha, \beta)+(1-\alpha)+x e^{-r t}\right]+\lambda[\alpha-\beta],
$$

where the Lagrange multiplier (or costate variable) $\lambda$ is also a function of $t$. Necessary conditions for a function $\alpha$ solving problem (4.1) are: 
(a) At each $t, \alpha$ maximizes $H$. $^{2}$ Thus, for an interior solution $0<\alpha<1$, we have $\partial H / \partial \alpha=0$, hence

$$
e^{-\rho t}[\partial g(\alpha, \beta) / \partial \alpha-1]+\lambda=0 .
$$

For a solution $\alpha=0$ we have

$$
e^{-\rho t}[\partial g(\alpha, \beta) / \partial \alpha-1]+\lambda \leq 0,
$$

and for a solution $\alpha=1$ we have

$$
e^{-\rho t}[\partial g(\alpha, \beta) / \partial \alpha-1]+\lambda \geq 0
$$

(b) $\dot{x}=\partial H / \partial \lambda$, i.e., $\dot{x}=\alpha-\beta$;

(c) $\dot{\lambda}=-\partial H / \partial x$, i.e., $\dot{\lambda}=-e^{-(\rho+r) t}$;

(d) Transversality condition: $\lim _{t \rightarrow \infty} \lambda(t)=0$.

Conditions (c) and (d) together imply

$$
\lambda(t)=\frac{e^{-(\rho+r) t}}{\rho+r},
$$

which may be substituted in the conditions formulated in (a).

The Hamiltonian and necessary conditions for problem (4.2) look similar and therefore will not be written down explicitly.

Observe that, in general, interior solutions cannot always be expected. For an interior solution for $\alpha$ (and fixed $\beta$ ), the appropriate condition under (a) becomes

$$
\frac{\partial g(\alpha, \beta)}{\partial \alpha}=1-\frac{e^{-r t}}{\rho+r}
$$

and, assuming that the partial derivative of $g$ with respect to $\alpha$ is nonnegative, this condition cannot be met for low values of $t$ if $\rho+r<1$. In that case $\alpha=1$ for low values of $t$. On the other hand, if $\rho+r \geq 1$, a necessary condition to have an interior solution $\alpha$ for all values of $t$ (and $\beta$ ) is that $r>0$ and the derivative $\partial g(\alpha, \beta) / \partial \alpha$ takes all values between $1-1 /(\rho+r)$ and 1 .

In the following subsections we consider a few specifications of $g$ and $h$ which enable us to derive exact solutions.

\footnotetext{
${ }^{2}$ In what follows it is convenient to suppress $t$. from the notation whenever this does not lead to confusion.
} 


\subsubsection{Bang-bang solutions}

The specification considered here allows "bang-bang" solutions, i.e., solutions taking only the values 0 and 1 , among the open-loop Nash equilibria. Let

$$
g(\alpha, \beta)=\alpha(1-\beta), \quad h(\alpha, \beta)=\beta(1-\alpha) .
$$

The conditions in (a)-(d) of the previous section lead to

$$
\begin{aligned}
& \alpha(t)=0 \text { if } \beta(t)>\frac{e^{-r t}}{\rho+r} \\
& \alpha(t)=1 \text { if } \beta(t)<\frac{e^{-r t}}{\rho+r},
\end{aligned}
$$

and analogous conditions for $\beta(t)$, depending on $\alpha(t)$. Further, $\alpha$ (or $\beta$ ) may take on arbitrary values between 0 and 1 if we have an equality sign in any of these conditions. This leads to the following description of open-loop Nash. equilibria. Here, $t^{\prime}$ is the value of $t$ for which $e^{-r t} /(\rho+r)=1$ and $t^{\prime \prime}$ is the value of $t$ for which $e^{-s t} /(\rho+s)=1$. Observe that $t^{\prime}\left\langle t^{\prime \prime}\right.$ if $r>s>0$, provided that $t^{\prime}$ and $t^{\prime \prime}$ exist.

Proposition 4.28 With the specifications $g(\alpha, \beta)=\alpha(1-\beta)$ and $h(\alpha, \beta)=$ $\beta(1-\alpha)$ and for $r>s>0$, the open-loop Nash equilibria are combinations of strategies $\alpha$ and $\beta$ containing only isolated discontinuities and satisfying:

i. For every $0 \leq t \leq t^{\prime}: \alpha(t)=\beta(t)=1$;

ii. For $t=t^{\prime}: \beta(t)=1$ and $\alpha(t)$ is arbitrary;

iii. For $t^{\prime}<t<t^{\prime \prime}: \beta(t)=1$ and $\alpha(t)=0$;

iv. For $t \geq t^{\prime \prime}: \beta(t)=1$ and $\alpha(t)=0$, or $\beta(t)=0$ and $\alpha(t)=1$, or $\beta(t)=\frac{e^{-r t}}{\rho+r}$ and $\alpha(t)=\frac{e^{-s t}}{\rho+r}$.

Thus, in this specification there are solutions taking on only the values 0 and 1. Solutions of this kind are usually called bang-bang solutions. Both players might start off (depending on the values of $r$ and $s$ relative to the common discount factor $\rho$ ) with full investment in the first (competitive) project. In the longer run, however, in equilibrium either one of the players invests fully in this project and the other one invests nothing or the investments of both 
players are between 0 and 1 but in the long run converge to 0 . It should be noted that the solutions in Proposition 4.28 are formulated at each point $t$ in time separately, so that the resulting strategies may be highly discontinuous. The first player to jump (necessarily) to zero investment is the one with the higher of the two rates $r$ and $s$ (as can be easily seen); at that point, it is no longer advantageous to compensate for the comparative "unlearning" effect of investment in the first project (given that the other player still invests fully) by also investing in that project. Thus, the player with the higher of the two rates $r$ and $s$ is the first one "to give up". A plausible equilibrium would be one where after this event this player stays at a zero investment level, while his opponent stays at investment level 1.

A proof of Proposition 4.28 can be based on the necessary conditions stated in the previous section and will not be elaborated.

\subsubsection{A Cobb-Douglas case}

In this subsection we assume specifications which also allow interior solutions of the players' maximization problems, that is, open-loop Nash equilibria with investments which may be strictly between 0 and 1 . To be precise, we take

$$
g(\alpha, \beta)=\sqrt{\alpha(1-\beta)}, \quad h(\alpha, \beta)=\sqrt{\beta(1-\alpha)} .
$$

The analysis of the general Cobb-Douglas case is more tedious but will not exhibit essentially different features.

The next proposition describes the open-loop Nash equilibria for the situation analogous to the one in Proposition 4.28. In order to make the description easier to digest, we first introduce some notation.

Assume $r>s>0$ and $\rho+r \leq 1$. Then let

$$
\begin{array}{rlrl}
t_{1} & =-\frac{\ln (\rho+r)}{r}, & t_{2} & =-\frac{\ln (\rho+s)}{s}, \\
t_{3}=-\frac{\ln \frac{1}{2}(\rho+r)}{r}, & t_{4}=-\frac{\ln \frac{1}{2}(\rho+s)}{s} .
\end{array}
$$

It can be verified that $0 \leq t_{1} \leq t_{2} \leq t_{4}$ and that $t_{1} \leq t_{3} \leq t_{4}$.

For $t \geq 0$ define

$$
\begin{aligned}
v(t)=1-\frac{e^{-r t}}{\rho+r}, & w(t)=1-\frac{e^{-s t}}{\rho+s}, \\
\alpha^{*}(t)=\frac{1-4 w^{2}(t)}{1-16 v^{2}(t) w^{2}(t)}, \quad \beta^{*}(t) & =\frac{1-4 v^{2}(t)}{1-16 v^{2}(t) w^{2}(t)} .
\end{aligned}
$$


We can now state our proposition.

Proposition 4.29 With the specifications $g(\alpha, \beta)=\sqrt{\alpha(1-\beta)}, h(\alpha, \beta)=$ $\sqrt{\beta(1-\alpha)}, r>s>0$, and $\rho+r \leq 1$, the open-loop Nash equilibria are combinations of strategies $\alpha$ and $\beta$ containing only isolated discontinuities and satisfying:

i. For every $0 \leq t \leq t_{1}: \alpha(t)=\beta(t)=1$;

ii. For $t=t_{1}: \beta(t)=1$ and $\alpha(t)$ is arbitrary;

iii. For $t_{1}<t<t_{2}: \beta(t)=1$ and $\alpha(t)=0$;

iv. For $t_{2} \leq t \leq t_{4}$ there are two cases:

a. If $t_{3} \leq t_{2}$, then $\beta(t)=1$ and $\alpha(t)=0$.

b. If $t_{2}<t_{3}<t_{4}$, then for $t_{2} \leq t \leq t_{3}$ there are three possibilities: $\beta(t)=1, \alpha(t)=0$, or $\beta(t)=0, \alpha(t)=1$, or $\beta(t)=\beta^{*}(t)$ and $\alpha(t)=\alpha^{*}(t)$, while for $t_{3}<t<t_{4}: \beta(t)=1, \alpha(t)=0$;

v. For $t \geq t_{4}: \beta(t)=\beta^{*}(t)$ and $\alpha(t)=\alpha^{*}(t)$.

Proposition 4.29 describes the most general case: in all other cases with $r \geq s$, the only difference may be that the whole picture moves to the left (or, equivalently, the origin to the right). Of course, the analysis of the case $r \leq s$ is similar. The proof of Proposition 4.29 is again based on the conditions formulated in the previous section, and will not be given in detail.

Figure 4.2 depicts some strategy combinations described by Proposition 4.29. Again, it should be noted that the strategies are defined for each. $t$ separately, and thus may contain any number of isolated discontinuities. In all cases, after $t_{4}$ the additional payoff effects associated with the state variables $x$ and $y$ decrease rapidly, causing player 1 to start investing again, while player 2 gradually decreases investments in the competitive project. In. the limit, both $\alpha^{*}$ and $\beta^{*}$ approach $\frac{1}{5}$.

The following proposition applies to the situation where one of the two players has a nonpositive depreciation rate of the (un)learning payoffs.

Proposition 4.30 Assume $g(\alpha, \beta)=\sqrt{\alpha(1-\beta)}, h(\alpha, \beta)=\sqrt{\beta(1-\alpha)}, r \leq$ $0, s>0$, and $\rho+r \leq 1$, and $|r|<\rho$. Let, as above, $t_{2}=-\ln (\rho+s) / s$. Then, for an open-loop Nash equilibria. we have: 

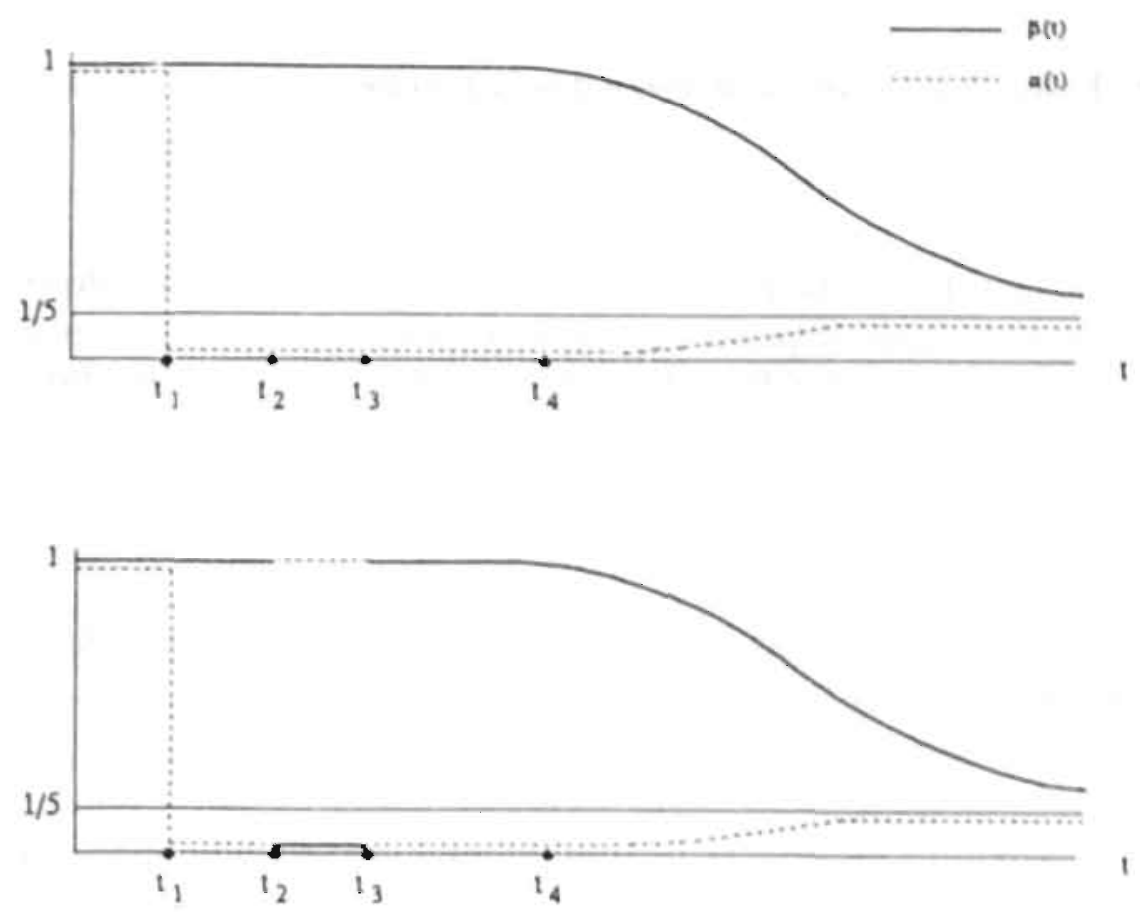

Figure 4.2: Proposition 4.29.

i. For all $t>\max \left\{0, t_{2}\right\}: \alpha(t)=1, \beta(t)=0$;

ii. For all $0 \leq t<t_{1}: \alpha(t)=\beta(t)=1$.

This proposition confirms the obvious intuition that the player with the nonpositive depreciation rate survives, as far as investment in the first project is concerned. If $\rho+r>1$, then in the longer run this will still hold although, initially, the equilibrium strategies may look different (details are omitted).

\subsubsection{An alternative formulation}

A drawback in the formulation of the investment problem in Subsection 4.4.1 is that the effects of the state variables $x$ and $y$ vanish in the long run due to the presence of the coefficients $e^{-r t}$ and $e^{-s t}$; there is not only relative but also absolute "unlearning" or "depreciation" as time goes on. ${ }^{3}$

\footnotetext{
${ }^{3}$ See Vega-Redondo [1994].
} 
To avoid this, we could alternatively require

$$
x(t)=\int_{0}^{t} k(t, \tau) f(\alpha(\tau), \beta(\tau)) d \tau,
$$

where, as before, $x$ is the state variable for player 1 , where $f$ describes how the state variable depends on the investment plans of both players, and where the function $k$ reflects the depreciation or growth of the state variable. Differentiating, we obtain

$$
\dot{x}(t)=\int_{0}^{t} \frac{\partial k(t, \tau)}{\partial t} f(\alpha(\tau), \beta(\tau)) d \tau+k(t, t) f(\alpha(t), \beta(t)) .
$$

In the special situation that $\partial k(t, \tau) / \partial t=l(t) k(t, \tau)$ for some function $l$ depending only on $t$, equation (4.4) implies

$$
\dot{x}(t)=x(t) l(t)+k(t, t) f(\alpha(t), \beta(t)) .
$$

Instead of maximization (4.1). now consider the maximization problem

$$
\begin{array}{ll}
\text { Maximize } & \int_{0}^{\infty} e^{-\rho t}[g(\alpha(t), \beta(t))+(1-\alpha(t))+x(t)] d t \\
\text { subject to } & \left\{\begin{array}{l}
\dot{x}(t)=x(t) l(t)+k(t, t) f(\alpha(t), \beta(t)) \\
x(0)=x_{0}, \alpha(t) \in[0,1] .
\end{array}\right.
\end{array}
$$

The coefficient $e^{-r t}$ has now been removed from the objective function; instead the state equation has been replaced by (4.5). A similar formulation can be given for player 2. The corresponding Hamiltonian is now given by

$$
\begin{aligned}
H(\alpha, x, t, \lambda)= & e^{-\rho t}[g(\alpha(t), \beta(t))+(1-\alpha(t))+x(t)] \\
& +\lambda(t)[x(t) l(t)+k(t, t) f(\alpha(t), \beta(t))],
\end{aligned}
$$

with

$$
\dot{\lambda}=-\frac{\partial H}{\partial x}=-e^{-\rho t}-l(t) \lambda(t)
$$

as the costate equation. Again, similar expressions hold for player 2.

A simple example is obtained by assuming, in line with the preceding subsections,

$$
f(\alpha(\tau), \beta(\tau))=\alpha(\tau)-\beta(\tau)
$$

and

$$
k(t, \tau)=e^{r(\tau-t)} .
$$


Then $l(t)=-r$ (we assume $r>0$ ), and it is easily established, also using the transversality condition $\lim _{t \rightarrow \infty} \lambda(t)=0$, that

$$
\lambda(t)=\frac{e^{-\rho t}}{r+\rho} .
$$

Substituting this expression for $\lambda(t)$ in the Hamiltonian and maximizing at a given $t$ and for a given strategy $\beta(t)$ of player 2 over the possible values of $\alpha(t)$, it follows easily that the maximizing value of $\alpha$ will be independent of $t$. In other words (and making similar assumptions about player 2), in an openloop Nash equilibrium the strategies of the players can be chosen constant over time, for this particular choice of the function $k$ (and the corresponding function for player 2). For particular choices of the functions $g$ and $h$ being the immediate payoff functions of players 1 and 2 respectively, from investing in the first project, such an open-loop Nash equilibrium can be calculated, for instance for Cobb-Douglas payoff functions as in the preceding sections. Details are left to the reader.

\subsection{References}

Acharya, S.N., 1981, Perspectives and problems of development in SubSaharan Africa, World Development 9, 109-146.

Arrow, K.J., 1962, The economic implications of learning by doing, Review of Economic Studies 29, 155-173.

Arrow, K.J., 1994, Comment on Joosten, Peters and Thuijsman, in "The Economics of Growth and Technical Change", G. Silverberg \& L. Soete (eds.), Edward Elgar, Aldershot, pp. 258-259.

Aumann, R.J., 1981, Survey of repeated games, in "Essays in Game Theory and Mathematical Economics in honor of Oskar Morgenstern", Bibliographisches Institut, Mannheim, pp. 11-42.

Blackwell, D., \& T.S. Ferguson, 1968, The big match, Annals of Mathematical Statistics 33, 719-726. 
Blanchard, O.J., \& L.H. Summers, 1986, Hysteresis and the European unemployment problem, in "NBER Macroeconomics Annual", S. Fisher (ed.), MIT Press, Cambridge, pp. 15-78.

Cheng, L., 1984, International competition in R\&D and technological leadership, Journal of International Economics 17, 15-40.

Filar, J.A., \& T.E.S. Raghavan, 1984, A matrix game solution of the single controller stochastic game, Mathematics of Operations Research 9, 356-362.

Gillette, D., 1957, Stochastic games with zero stop probabilities, in "Contributions to the Theory of Games III", Annals of Mathematical Studies 39, 179-187.

Joosten, R., H. Peters, \& F. Thuijsman, 1994, Games with changing payoffs, in "The Economics of Growth and Technical Change", G. Silverberg \& L. Soete (eds.), Edward Elgar, Aldershot, pp. 244-257.

Joosten, R., H. Peters, \& F. Thuijsman, 1995, Unlearning by not-doing: Repeated games with vanishing actions, Games and Economic Behavior 9, $1-7$.

Levhari, D., \& L. Mirman, 1980, The great fish war, Bell Journal of Economics 10, 322-344.

Mc Millan, J., 1986, "Game Theory in International Economics", Harwood Academic Publishers, Chur.

Mertens, J.F., \& A. Neyman, 1981, Stochastic games, International Journal of Game Theory 10, 53-66.

Möller, J., 1990, Unemployment and deterioration of human capital, Empirical Economics 15, 199-215.

Raghavan, T.E.S., \& J.A. Filar, 1991, Algorithms for stochastic games: A survey, Zeitschrift für Operations Research 35, 437-472. 
Reinganum, J., 1982, A dynamic game of R\&D: Patent protection and competition behavior, Econometrica 50, 671-688.

Shapley, L.S., 1953, Stochastic games, Proceedings of the National Academy of Sciences U.S.A. 39, 1095-1100.

Spence, A.M., 1979, Investment strategy and growth in a new market, Bell Journal of Economics 10, 1-19.

Starr, A.W., \& Y.C. Ho, 1969a, Nonzero-sum differential games, Journal of Optimization Theory and Applications 3, 184-206.

Starr, A.W., \& Y.C. Ho, 1969b, Further properties of nonzero-sum differential games, Journal of Optimization Theory and Applications 3, 207-219.

Thuijsman, F., 1992, "Optimality and Equilibria in Stochastic Games", CWI-tract 82, Centre for Mathematics and Computer Science, Amsterdam.

Vega-Redondo, F., 1994, Comment on Joosten, Peters and Thuijsman, in "The Economics of Growth and Technical Change", G. Silverberg \& L. Soete (eds.), Edward Elgar, Aldershot, pp. 258-259.

Vrieze, O.J., 1981, Linear programming and undiscounted stochastic games in which one player controls transitions, OR Spektrum 3, 29-35. 



\section{Chapter 5}

\section{Egalitarianism, potentials, and values}

\subsection{Introduction}

The Shapley value (Shapley [1953]) is probably the best known solution for transferable utility games. One of the characterization of the Shapley value involves the following four axioms: efficiency, symmetry, additivity, and the null-player property. A value is efficient if it distributes for every game the worth of the grand coalition among all players. A value is symmetric if it gives the same utility to any pair of players whose marginal contributions in any game are identical. A value is additive if it gives to each player in any game being the sum of two games, precisely the sum of the utilities which the value gives him in the two separate games. A value has the null-player property if every player whose marginal contribution to all coalitions equals zero, receives a utility of zero.

Young [1985] proves that the Shapley value is the unique solution satisfying the axioms of efficiency, symmetry and strong monotonicity. Strong monotonicity compares the utilities attributed by a value in different games with the same player set. Consider two games with the same player set, and assume that some player's marginal contributions in the first game are at least equal to his marginal contributions in the second game. Then strong monotonicity implies that the utility given by the value to the player in the first game is at least the utility given to him in the second game.

Hart \& Mas-Colell [1989] characterize the Shapley value by the axioms of 
consistency and standardness. A reduced game is a game on a subset of the player set, that remains after compensating all other players in the original game in an appropriate way (which may vary with the solution in question). Then, consistency requires that when a solution is applied to the reduced game each player in the reduced game receives the same utility as in the original game. Standardness implies that when the solution is applied to an arbitrary two-person game, each player receives half of the surplus of that game on top of the amount that he receives in the one-person coalition. Hart \& Mas-Colell [1989] extend this result to the entire family of weighted Shapley values: Each weighted Shapley value is uniquely determined by $\mathrm{H}$ (art-\&) $\mathrm{M}$ (as-Colell)-consistency and the amounts attributed in two-person games.

We introduce the axiom of $\alpha$-egalitarianism, which requires that a value attributes to each null-player in a game, a fraction $\alpha$ of the per-capita income. We interpret the real number $\alpha$ as a proportional tax on every coalition in the game, which is to be equally redistributed among all of its members. Thus, $\alpha$ reflects a social norm, i.e., the level of egalitarianism or solidarity in the society. Social norms on equality and solidarity exist in real. life, and one may wish to devise solutions which incorporate these aspects. Several contributions explore a similar theme, e.g., Nowak \& Radzik [1994], Dutta \& Ray [1989]. Here, we follow a different, approach.

We show that for fixed $\alpha$, the axioms of efficiency, symmetry, additivity, and $\alpha$-egalitarianism uniquely determine a value, the $\alpha$-egalitarian Shapley value. We introduce two further axioms, namely equal coalitional improvement and $\alpha$-marginality. Suppose that in a game a coalition, say $T$, were to find a way to work more efficiently, such that its worth, as well as the worth of each coalition containing $T$, is raised by a fixed amount. Then, equal coalitional improvement implies that all members of $T$ improve equally under the value. The property of $\alpha$-marginality is a generalization of marginality and requires the following. Take two games with the same player sets and suppose a player's marginal contribution to any coalition not containing him are identical in these two games. Apply the value to both games, then the utilities to this player in the two games above the fraction $\alpha$ of the per-capita income of the separate games, are the same. (Marginality is 0 -marginality and follows immediately from strong monotonicity, cf. Young [1985].) With these new axioms we give two more axiomatic characterizations of the $\alpha$-egalitarian Shapley values. The first characterization is by the axioms of efficiency, triviality (Chun [1989]), equal coalitional improvement, and $\alpha$-marginality. The second characterization is by the axioms of efficiency, 
symmetry, and $\alpha$-marginality.

Our final characterization of the $\alpha$-egalitarian Shapley value follows Hart $\&$ Mas-Colell [1989]. We introduce $\alpha$-dependent variants of consistency and standardness, which are linked to the level of egalitarianism in the society. We call these new axioms $\alpha$-consistency and $\alpha$-standardness, respectively. For fixed $\alpha$, we prove that these two axioms characterize the $\alpha$-egalitarian Shapley value.

Hart \& Mas-Colell [1989] use potentials in their characterization of the weighted Shapley values. A potential in this context is a map which assigns to each transferable utility game a unique real number. We take the idea of 'taxing and redistributing' one step further to derive a family of potentials and a family of values depending on a tuple of parameters $(a, b, \alpha)$, where $a, b$ are vectors of weights and $\alpha$ represents the level of egalitarianism. A value in this family will be denoted by $\Phi^{a, b, \alpha}$, and all values of this form satisfy efficiency and linearity. The weighted Shapley values, the $\alpha$-egalitarian Shapley values, and the egalitarian value are special examples of such values. We investigate relations between restrictions on $(a, b, \alpha)$ and properties of the value $\Phi^{a, b, a}$.

In Section 5.2 we introduce the model and present characterizations of the (weighted) Shapley value(s) in order to compare our results with these characterizations. Section 5.3 is devoted to $\alpha$-egalitarian Shapley values and several axiomatic characterizations of these values. In Section 5.4 we introduce and examine the families of $(a, b, \alpha)$-potentials and values $\Phi^{a, b, a}$.

\subsection{The model and preliminaries}

Let $\Re$ denote the set of real numbers. Let $Z$ be a nonempty set of natural numbers, representing the set of potential players. (Strict) inclusions are denoted by $(\subset) \subseteq$. A coalition is a finite subset of $Z$. A transferable utility game is a pair $(N, v)$ where $N \subseteq Z$ is a coalition and $v: 2^{N} \rightarrow \Re$, with $v(\emptyset)=0$. The function $v$ is the characteristic function. We denote the set of all games with player set $N$ by $G^{N}$, the set of all games is $G$.

Let $(N, v) \in G$, then:

- For $M \subseteq N$, the characteristic function of the game $(M, v)$ is the map $v$ restricted to $2^{M}$; 
- Player $i \in N$ is a null-player in $(N, v)$ if $v(S \cup\{i\})=v(S)$ for all $S \subset N$;

- The set $\mathcal{N}(N, v)$ is the set of null-players in $(N, v)$;

- Players $i, j \in N$ are symmetric in $(N, v)$, if $v(S \cup\{i\})=v(S \cup\{j\})$ for all $S \subseteq N \backslash\{i, j\}$;

- The marginal contribution (Young [1985]) of $i \in N$ to $S \subseteq N$ in $(N, v)$ is given by

$$
\Delta_{i}^{v}(S)=\left\{\begin{array}{ll}
v(S)-v(S \backslash\{i\}) & \text { if } i \in S \\
v(S \cup\{i\})-v(S) & \text { if } i \notin S
\end{array} .\right.
$$

Let $v, w: 2^{N} \rightarrow \Re$, and $\alpha, \lambda \in \Re$, then:

- $(v+w)(S)=v(S)+w(S)$, for all $S \subseteq N$,

- $(\lambda v)(S)=\lambda v(S)$ for all $S \subseteq N$,

- $\left(v^{\alpha}\right)(S)=(1-\alpha) v(S)$ for all $S \subseteq N$.

With the first two operations $G^{N}$ is a linear space. We call the game $\left(N, v^{\alpha}\right)$ the $\alpha$-taxed game of $(N, v)$, i.e., the game which remains if a proportional $\operatorname{tax}$ of $\alpha$ is levied on the worth of each coalition in the game $(N, v)$. For arbitrary $N \subseteq Z$, let $\left(N, v_{0}\right)$ denote the zero-game, i.e., $\mathcal{N}\left(N, v_{0}\right)=N$. For nonempty $T \subseteq N \subseteq Z$, the $T$-unanimity game $\left(N, u_{T}\right)$ is the game with $u_{T}(S)=1$ if $T \subseteq S \subseteq N$, and $u_{T}(S)=0$ otherwise.

Shapley [1953] demonstrated that the collection of all $T$-unanimity games $(\emptyset \neq T \subseteq N)$ constitutes a basis of the linear space $G^{N}$. Hence, for every game $(N, v)$ with nonempty player-set $N$, there exists a unique set of numbers $\left\{c_{T} \in \Re \mid \emptyset \neq T \subseteq N\right\}$, satisfying

$$
v=\sum_{\emptyset \neq T \subseteq N} c_{T} u_{T}
$$

Explicit formulas for these numbers $c_{T}$ are given by

$$
c_{T}=\sum_{0 \neq S \subseteq T}(-1)^{|T|-|S|} v(S) .
$$


A value is a map $\psi$ assigning to each game $(N, v)$, a vector in $\Re^{N}$. The interpretation is that if the value is applied to a game $(N, v)$, the $i$-th component of the vector represents the utility attributed to player $i \in N$ in the game $(N, v)$. Let $\psi$ be a value, then:

- $\psi$ is efficient if $\sum_{i \in N} \psi_{i}(N, v)=v(N)$ for every $(N, v) \in G$;

- $\psi$ is symmetric if $\psi_{i}(N, v)=\psi_{j}(N, v)$ whenever $i, j \in N$ are symmetric players in $(N, v) \in G$;

- $\psi$ is anonymous if $\psi_{\pi i}(N, \pi v)=\psi_{i}(N, v)$ for all $(N, v) \in G, i \in N$, and all permutations $\pi$ of $N$, where $\pi v(S)=v(\pi S)$ for all $S \subseteq N$;

- $\psi$ is additive if $\psi(N, v+w)=\psi(N, v)+\psi(N, w)$ for all $(N, v),(N, w) \in$ $G$;

- $\psi$ is linear if $\psi(N, \lambda v+\mu w)=\lambda \psi(N, v)+\mu \psi(N, w)$ for all $(N, v),(N, w) \in$ $G$, and all $\lambda, \mu \in \Re$;

- $\psi$ satisfies the null-player property if $\psi_{i}(N, v)=0$ whenever $i \in$ $\mathcal{N}(N, v)$

- $\psi$ is standard if $\psi_{i}(\{i, j\}, v)=v(\{i\})+\frac{v(\{i, j\})-v(\{i\})-v(\{j\}}{2}$ for all twoperson games $(\{i, j\}, v) \in G$;

- $\psi$ is trivial (Chun [1989]) if $\psi_{i}\left(N, v_{0}\right)=0$ for all $N \subseteq Z, i \in N$;

- $\psi$ is strongly monotonic (Young [1985]) if for any pair of games $(N, v),(N, w) \in G$, and any $i \in N$, it holds that if $\Delta_{i}^{v}(S) \geq \Delta_{i}^{w}(S)$ for all $S \subseteq N$, then $\psi_{i}(N, v) \geq \psi_{i}(N, w)$;

- $\psi$ satisfies marginality (Young [1985]) if for any pair of games $(N, v),(N, w) \in$ $G$, and any $i \in N$, it holds that if $\Delta_{i}^{v}(S)=\Delta_{i}^{w}(S)$ for all $S \subseteq N$, then $\psi_{i}(N, v)=\psi_{i}(N, w)$.

For an additive value the analysis may be restricted to its solutions of scalar multiples of all unanimity games, as $\psi(N, v)=\psi\left(N, \sum_{\emptyset \neq T \subseteq N} C_{T} u_{T}\right)=$ $\sum_{\emptyset \neq T \subseteq N} \psi\left(N, c_{T} u_{T}\right)$ for all additive values $\psi$ and $(N, v) \in G$.

An example of a value is the egalitarian value denoted by $\eta_{i}$ in the sequel. For arbitrary $(N, v) \in G, \eta_{i}(N, v)=\frac{v(N)}{|N|}$ for all $i \in N$. The egalitarian value is efficient, symmetric, additive, linear, and trivial, but it is not standard and it does not satisfy the null-player property. 
A value which has received much attention, is the Shapley value (cf. Shapley [1953], Roth [1988]), denoted by $\varphi$ in the sequel. For every $(N, v) \in$ $G$ and every $i \in N, \varphi$ is given by

$$
\varphi_{i}(N, v)=\sum_{S \subseteq N \backslash\{i\}} \frac{|S| !(|N|-|S|-1) !}{|N| !} \Delta_{i}^{v}(S) .
$$

The interpretation of the formula is that the Shapley value assigns to each player his average marginal contribution in the game. It may be verified that the Shapley value satisfies efficiency, anonymity, symmetry, additivity, the null-player property, linearity, standardness, triviality, strong monotonicity, and marginality.

An equivalent notation of the Shapley value is the following. For every $(N, v) \in G$, and every $i \in N, \varphi$ is given by

$$
\varphi_{i}(N, v)=\sum_{T \subseteq N: T \ni i} \frac{c_{T}}{|T|} \text { for all } i \in N,
$$

where the numbers $c_{T}$ are given by (5.1). This may be seen by noting that in every game $c_{T} u_{T}(\emptyset \neq T \subseteq N)$ all players in $T$ receive $\frac{c_{T}}{|T|}$, all players outside $T$ get zero. Note furthermore that the Shapley value satisfies additivity, hence by the remarks above lead to this alternative notation.

The following axiomatic characterization of the Shapley value is a variation of Shapley's [1953] axiomatic characterization.

Proposition 5.1 Let $\psi$ be a value. Then, the following two statements are equivalent:

i. $\psi$ satisfies efficiency, symmetry, additivity, and the null-player property;

ii. $\psi=\varphi$.

Many characterizations of the Shapley value have been formulated using alternative axioms. The following axiomatic characterization of this solution is based on marginal contributions.

Proposition 5.2 (Young [1985]) Let $\psi$ be a value. Then, the following two statements are equivalent: 
i. $\psi$ is efficient, anonymous, and strongly monotonic;

ii. $\psi=\varphi$.

Note that strong monotonicity implies marginality. In fact, Young uses marginality in his proof of Proposition 5.2.

Let $w \in \Re^{Z}$ be a vector of exogenously given weights satisfying $w_{i}>0$ for all $i \in Z$. Then, the weighted Shapley value (cf., Shapley [1953], Kalai \& Samet [1987]), denoted by $\varphi^{w}$ in the sequel, is for every $(N, v) \in G$ given by

$$
\varphi_{i}^{w}(N, v)=\sum_{T \subseteq N: T \ni i} c_{T}\left(\frac{w_{i}}{\sum_{j \in T} w_{j}}\right) \text { for all } i \in N .
$$

For $w \in \Re^{Z}$ satisfying $w_{i}=w_{j}>0$ for all $i, j \in Z$, we have $\varphi^{w}=\varphi$. Each weighted Shapley value satisfies the axioms of efficiency, additivity and the null-player property. It is characterized (up to a scalar multiple in $w$ ) by these three axioms and the amounts which in all $T$-unanimity games, $\emptyset \neq T \subseteq N$, are attributed to the members of $T$.

Symmetric players are not treated equally if $w_{i} \neq w_{j}$ for some $i, j \in Z$. The following interpretation for asymmetric weights of the weighted Shapley values is similar to the one given by Kalai \& Samet [1987]. Suppose players are to join in a project, and they can generate positive profits if they all cooperate, and can generate zero-profits otherwise. Then, the Shapley value gives each player an equal share of the profits. This seems reasonable when players have to provide similar inputs. In case, however, that there is an asymmetry in the efforts put forward by the players necessary to complete the project, a symmetric division of the profits may not be reasonable. Dividing the profits proportional to the efforts put forward by the players, seems a good alternative. For this purpose, the weighted Shapley value may be used with the weights equal to the effort-levels.

Hart \& Mas-Colell [1989] introduce the following family of 'potentials'.

Definition 5.3 (Hart \& Mas-Colell (1989]) Let $w \in \Re^{Z}$ satisfy $w_{i}>0$ for all $i \in Z$. Then the $w$-potential is the map $P_{w}: G \rightarrow \Re$ satisfying:

i. $P_{w}^{\prime}(\emptyset, v)=0$,

ii. $\sum_{i \in N} w_{i}\left[P_{w}(N, v)-P_{w}(N \backslash\{i\}, v)\right]=v(N)$ for every $(N, v) \in G$. 
So, a $w$-potential is a map attributing to each game a unique real number. The condition of strict positivity on the vector of weights $w$ guarantees that the $w$-potential is well-defined and unique. The weighted Shapley value $\varphi^{w}$ (cf., Shapley [1953], Kalai \& Samet [1987]) is connected to this $w$-potential as follows (cf., Hart \& Mas-Colell [1989]):

$$
\varphi_{i}^{w}(N, v)=w_{i}\left[P_{w}(N, v)-P_{w}(N \backslash\{i\}, v)\right] \text { for all }(N, v) \in G, i \in N .
$$

The $w$-potential provides an algorithm to compute the corresponding weighted Shapley value $\varphi^{w}$, by using:

$$
P_{w}(S, v)=\frac{v(S)+\sum_{k \in S} w_{k} P_{w}(S \backslash\{k\}, v)}{\sum_{k \in S} w_{k}} \text { for all nonempty } S \subseteq N .
$$

Furthermore, $w$-potentials are useful in proving HM-consistency of a value as Hart \& Mas-Colell [1989] have shown. As mentioned before consistency is a reduced-game property. It may be described as follows. Let $\psi$ be a value. For any group of players in a game, one defines a reduced game among them by giving the rest of the players the payoffs according to $\psi$. Then $\psi$ is called consistent if, when applied to any reduced game, it yields the same payoffs as in the original game. One way to formalize this reduced-game property is the following.

Definition 5.4 (Hart \& Mas-Colell [1989]) Let $\psi$ be a value, $(N, v) \in G$, and $\emptyset \neq U \subset N$. Then the $(\mathbf{U}, \psi)$-realuced game of $\mathbf{v}$ is the game $v^{U, \psi}$ satisfying:

$$
\begin{aligned}
& \text { i. } v^{U, \psi}(S)=v(S \cup U)-\sum_{k \in U} \psi_{k}(S \cup U, v) \text { for all } \emptyset \neq S \subseteq N \backslash U ; \\
& \text { ii. } v^{U, \psi}(\emptyset)=0 .
\end{aligned}
$$

Definition 5.5 (Hart \& Mas-Colell [1989]) Let $\psi$ be a value. Then $\psi$ is $\boldsymbol{H M}$-consistent if for all games $(N, v) \in G$ and all $\emptyset \neq U \subset N$ :

$$
\psi_{i}\left(N \backslash U, v^{U, \psi}\right)=\psi_{i}(N, v) \text { for all } i \in N \backslash U .
$$

One can directly show with these definitions that the egalitarian value $\eta$ is HM-consistent. The following pertains to the utilities attributed by a value to the players in two-person games. 
Definition 5.6. (Hart \& Mas-Colell /1989]) Let $\psi$ be a value, and let $w \in \Re^{Z}$ satisfy $w_{i}>0$ for all $i \in Z$. Then $\psi$ is $w$-proportional if for all 2-person games $(\{i, j\}, v)$, it holds that $\psi_{i}(\{i, j\}, v)=\frac{w_{i} v(\{i, j\})-w_{i} v((j))+w_{j} v(\{i\})}{w_{i}+w_{j}}$.

In case that $w_{i}=c>0$ for all $i \in Z, w$-proportionality is equivalent to standardness. So, the weighted Shapley value with weights $w$, is $w$-proportional. Hart \& Mas-Colell [1989] prove the following axiomatic characterization.

Proposition 5.7 (Hart \& Mas-Colell [1989]) Let $\psi$ be $a$ value, and let $w \in$ $\Re^{Z}$ satisfy $w_{i}>0$ for all $i \in Z$. Then, the following two statements are equivalent:

i. $\psi$ is HM-consistent and w-proportional;

ii. $\psi=\varphi^{w}$.

\section{$5.3 \quad \alpha$-Egalitarian Shapley values}

All weighted Shapley values satisfy the null-player property. So do several other values in the literature, e.g., the nucleolus (Schmeidler [1969]) and the $\tau$-value (Tijs [1981]). However, instead of the null-player property, we would like to introduce the property of $\alpha$-egalitarianism. Recall that $\mathcal{N}(N, v)$ is the set of null-players in $(N, v)$, and let $\overline{\psi(N, v)}=\frac{\sum_{j \in N} \psi_{j}(N, v)}{|N|}$ be the per-capita income under the value $\psi$ in $(N, v)$.

Definition 5.8 Let $\alpha \in \Re$. The value $\psi$ is $\alpha$-egalitarian if, for every game $(N, v)$ and every $i \in \mathcal{N}(N, v), \psi_{i}(N, v)=\alpha \overline{\psi(N, v)}$.

$\alpha$-Egalitarianism states that each null-player in each game obtains the fixed fraction $\alpha$ of the per-capita income. We define the following family of $\alpha$ egalitarian values.

Definition 5.9 Let $\alpha \in \Re$. The $\alpha$-egalitarian Shapley value $\psi^{\alpha}$ is for every $(N, v) \in G$ given by $\psi^{\alpha}(N, v) \equiv(1-\alpha) \varphi(N, v)+\alpha \eta(N, v)$.

This section deals with characterizations of $\alpha$-egalitarian Shapley values for fixed $\alpha \in \Re$, namely Propositions 5.10, 5.13, 5.14, and 5.22. Propositions $5.10,5.14$, and 5.22 are inspired by the characterizations of the Shapley value presented as Propositions 5.1, 5.2, and 5.7. We begin by giving a characterization in the spirit of Shapley [1953]. 
Proposition 5.10 Let $\alpha \in \Re$ and let $\psi$ be a value. Then, the following two statements are equivalent:

i. $\psi$ is efficient, additive, symmetric, and $\alpha$-egalitarian;

ii. $\psi=\psi^{\alpha}$.

The proof of Proposition 5.10 relies on a standard analysis of the $T$-unanimity games $(\emptyset \neq T \subseteq N)$ and is left to the reader. Recall that in Proposition 5.1 the Shapley value is characterized by efficiency, symmetry, additivity, and the null-player property. So, replacing the null-player property in this set of four axioms by the axiom of $\alpha$-egalitarianism yields the $\alpha$-egalitarian Shapley value for fixed $\alpha \in \Re$.

In the following we firstly give two new definitions (axioms).

Definition 5.11 The value $\psi$ satisfies equal coalitional improvement if for all games $(N, v),(N, w)$ and any nonempty coalition $T \subseteq N$ with the property

- $w(S)=v(S)+c$ for some $c \in \Re$, and all $S \supseteq T$,

- $w(S)=v(S)$ otherwise,

there exists some $\tilde{c} \in \Re$ satisfying $\psi_{i}(N, w)-\psi_{i}(N, v)=\tilde{c}$ for all $i \in T$.

Suppose that a coalition $\emptyset \neq T \subseteq N$ has a gain such that the worths of all coalitions containing $T$ increase by the amount $c$. Then, the property of equal coalitional improvement requires that all members of $T$ improve by an amount $\tilde{c}$ under the value. The Shapley value and the egalitarian value satisfy equal coalitional improvement. So, an $\alpha$-egalitarian Shapley value must also satisfy this property. Weighted Shapley values however, do not satisfy this property in general.

Definition 5.12 The value $\psi$ satisfies $\alpha$-marginality if for all $(N, v),(N, w) \in$ $G$, and every null-player $i$ in $(N, v-w)$ :

$$
\psi_{i}(N, v)-\alpha \overline{\psi(N, v)}=\psi_{i}(N, w)-\alpha \overline{\psi(N, w)} .
$$


This property is an $\alpha$-dependent variant of the axiom of marginality, which has been discussed in the introduction and in Section 5.2. The axiom of $\alpha$-marginality compares the utilities attributed by a value in different games, requiring that if the vector of marginal contributions of a player is the same in two games, then the amounts which he receives on top of the fixed fraction $\alpha$ of the per-capita income in the two games, are identical.

The following characterization uses both the axioms of equal coalitional improvement and $\alpha$-marginality.

Proposition 5.13 Let $\alpha \in \Re$ and let $\psi$ be a value. Then, the following two statements are equivalent:

i. $\psi$ satisfies efficiency, triviality, equal coalitional improvement, and $\alpha$-marginality;

ii. $\psi=\psi^{\alpha}$.

Proof. The part that (ii) implies (i), is straightforward.

Let $\psi$ satisfy the four axioms in (i). Then by triviality $\psi\left(N, v_{0}\right)=\psi^{\alpha}\left(N, v_{0}\right)$ for all $\left(N, v_{0}\right) \in G$.

We now prove that $\psi(N, w)=\psi^{\alpha}(N, w)$ implies $\psi\left(N, w+c u_{T}\right)=\psi^{\alpha}(N, w)+$ $\psi^{\alpha}\left(c u_{T}\right)$, for arbitrary $(N, w) \in G$, nonempty $T \subseteq N$, and $c \in \Re$. This will complete the proof of the proposition in view of the representation of a game on the basis of unanimity games. Note that

$$
\begin{aligned}
& \psi_{i}^{\alpha}\left(N, c u_{T}\right)=\frac{(1-\alpha) c}{|T|}+\frac{\alpha c}{|N|} \text { for } i \in T, \\
& \psi_{i}^{\alpha}\left(N, c u_{T}\right)=\frac{\alpha c}{|N|} \text { for } i \in N \backslash T .
\end{aligned}
$$

Let $c \in \Re,(N, w) \in G$, and let $T \subseteq N$ be nonempty. Then, $\alpha$-marginality and efficiency of the value $\psi$ applied to $w$ and $w+c u_{T}$ imply

$$
\psi_{i}\left(N, w+c u_{T}\right)=\psi_{i}(N, w)+\alpha \frac{c}{|N|} \text { for all } i \in N \backslash T .
$$

By efficiency and equal coalitional improvement of $\psi$, we obtain

$$
w(N)+c=\sum_{i \in N \backslash T}\left(\psi_{i}(N, w)+\alpha \frac{c}{|N|}\right)+\sum_{i \in T}\left(\psi_{i}(N, w)+\tilde{c}\right),
$$


with $\tilde{c}=\frac{(1-\alpha) c}{|T|}+\frac{\alpha c}{|N|}$. Hence,

$$
\psi_{i}\left(N, w+c u_{T}\right)=\psi_{i}(N, w)+\frac{(1-\alpha) c}{|T|}+\frac{\alpha c}{|N|} \text { for all } i \in T .
$$

Then, $\psi\left(N, w+c u_{T}\right)=\psi^{\alpha}(N, w)+\psi^{\alpha}\left(c u_{T}\right)=\psi^{\alpha}\left(N, w+c u_{T}\right)$.

Proposition 5.13 yields an alternative characterization of the Shapley value by taking $\alpha=0$. The following result uses $\alpha$-marginality as well, but now in a similar manner as the property of marginality is used in the proof of the characterization of the Shapley value by Young [1985] (cf. Proposition 5.2).

Proposition 5.14 Let $\alpha \in \Re$ and let $\psi$ be a value. Then, the following two statements are equivalent:

i. $\psi$ satisfies efficiency, symmetry, and $\alpha$-marginality;

ii. $\psi=\psi^{\alpha}$.

Proof. We only prove that (i) implies (ii). The other implication is straightforward.

Let $\psi$ satisfy efficiency, symmetry and $\alpha$-marginality. Efficiency and symmetry imply triviality, hence $\psi_{i}\left(N, v_{0}\right)=0$ for all $i \in N$. So, $\psi\left(N, v_{0}\right)=$ $\psi^{\alpha}\left(N, v_{0}\right)$. Then, for nonempty $T \subseteq N$, and for all $c \in \Re$, we obtain

$$
\psi_{i}\left(N, c u_{T}\right)=\psi_{i}\left(N, v_{0}\right)+\frac{\alpha c}{|N|}=\frac{\alpha c}{|N|} \text { for all } i \in N \backslash T .
$$

The first equality follows since all players $i \in N \backslash T$ are null-players in both games $v_{0}$ and $c u_{T}$, and by $\alpha$-marginality. The second equality follows from triviality. Observe that all players in $T$ are symmetric players, hence symmetry and efficiency imply

$$
\psi_{i}\left(N, c u_{T}\right)=\frac{(1-\alpha) c}{|T|}+\frac{\alpha c}{|N|} \text { for all } i \in T .
$$

So, $\psi\left(N, c u_{T}\right)=\psi^{\alpha}\left(N, c u_{T}\right)$. Now, take the game $(N, v)$ and write $v=$ $\sum_{\emptyset \neq T \subseteq N} c_{T} u_{T}$ in a unique way. We show $\psi(N, v)=\psi^{\alpha}(N, v)$ by induction on the number of nonzero terms $c_{T}$ in $\sum_{\emptyset \neq T \subseteq N} c_{T} u_{T}$, and we call this number $\beta(v)$. Note that so far, we have shown for $\beta(v)=0$ and for $\beta(v)=1$, 
that $\psi(N, v)=\psi^{\alpha}(N, v)$. Assume, $\psi(N, z)=\psi^{\alpha}(N, z)$ for all $z \in G^{N}$ with $\beta(z)<k$, where $k \geq 2$. Let $(N, v)$ satisfy $\beta(v)=k$. Then there exist coalitions $T_{1}, T_{2}, \ldots, T_{k}$ and nonzero real numbers $c_{1}, c_{2}, \ldots, c_{k}$ such that $v=\sum_{r=1}^{k} c_{T r} u_{T r}$. Let $D=\bigcap_{r=1}^{k} T_{r}$. For $i \in N \backslash D$, we define the game $w^{i}=\sum_{r i \in T_{r}} c_{T_{r}} u_{T_{r}}$. Since $\Delta_{i}^{v}(S)=\Delta_{i}^{w^{i}}(S)$ for all $S \subseteq N$, we have $\psi_{i}(N, v)-\alpha \overline{\psi(N, v)}=\psi_{i}\left(N, w^{i}\right)-$ $\alpha \overline{\psi\left(N, w^{i}\right)}$, by $\alpha$-marginality. So, for all $i \in N \backslash D$ :

$$
\begin{aligned}
\psi_{i}(N, v)-\alpha \overline{\psi(N, v)} & =\psi_{i}\left(N, w^{i}\right)-\alpha \overline{\psi\left(N, w^{i}\right)} \\
& =\psi_{i}^{\alpha}\left(N, w^{i}\right)-\alpha \overline{\psi^{\alpha}\left(N, w^{i}\right)} \\
& =\psi_{i}^{\alpha}(N, v)-\alpha \overline{\psi^{\alpha}(N, v)} .
\end{aligned}
$$

The second equality holds since $\beta\left(w^{i}\right)<k$, hence we have $\psi\left(N, w^{i}\right)=$ $\psi^{\alpha}\left(N, w^{i}\right)$. So, $\psi_{i}(N, v)=\psi_{i}^{\alpha}(N, v)$ for all $i \in N \backslash D$, which in turn implies with efficiency, that

$\sum_{i \in D} \psi_{i}(N, v)=v(N)-\sum_{i \in N \backslash D} \psi_{i}(N, v)=v(N)-\sum_{i \in N \backslash D} \psi_{i}^{\alpha}(N, v)=\sum_{i \in D} \psi^{\alpha}(N, v)$,

Note that all players in $D$ are symmetric players, hence symmetry of $\psi$ and $\psi^{\alpha}$ implies

$$
\psi_{i}(N, v)=\frac{\sum_{i \in D} \psi_{i}(N, v)}{|D|}=\frac{\sum_{i \in D} \psi_{i}^{\alpha}(N, v)}{|D|}=\psi_{i}^{\alpha}(N, v) \text { for all } i \in D .
$$

Hence, $\psi(N, v)=\psi^{\alpha}(N, v)$ for all $(N, v)$ with $\beta(v)=k$.

We conclude this section by characterizing each $\alpha$-egalitarian Shapley value, similar to the characterization of the Shapley value by Hart \& MasColell [1989], namely by $\alpha$-dependent variants of consistency and standardness. For this purpose we introduce the following reduced game.

Definition 5.15 Let $(N, v) \in G, \alpha \in \Re$, and let $\psi$ be a value. For nonempty $U \subset N$, the $(\mathbf{U}, \psi, \alpha)$-reduced game $\left(\mathbf{N} \backslash \mathbf{U}, \mathbf{v}^{\mathbf{U}, \psi, \alpha}\right)$ of $v$ is given by

i. $v^{U, \psi, \alpha}(S)=v(S \cup U)-\sum_{k \in U} \psi_{k}(S \cup U, v)$, if $\alpha=1$ and $S \neq \emptyset$, or if $S=N \backslash U$;

ii. $v^{U, \psi, \alpha}(S)=$

$$
v(S \cup U)-\sum_{k \in U} \psi_{k}(S \cup U, v)+\frac{\alpha}{1-\alpha}\left[\frac{|U|}{|S|+|U|} v(S \cup U)-\sum_{k \in U} \psi_{k}(S \cup U, v)\right]
$$


if $\alpha \neq 1$ and $\emptyset \neq S \subset N \backslash U$;

iii. $v^{U, \psi, \alpha}(\emptyset)=0$.

For $\alpha=0$ or $\alpha=1$ this reduced game is equivalent to the $\mathrm{H}$ (art and) M(asColell)-reduced game (cf. Definition 5.4). Note that for all games $(N, v)$, it holds that $\frac{|U|}{|S|+|U|} v(S \cup U)-\sum_{k \in U} \eta_{k}(S \cup U, v)=0$, hence for the egalitarian value $\eta$, Definitions 5.4 and 5.15 are equivalent for arbitrary $\alpha \in \Re$.

We now generalize 'consistency' and 'standardness'.

Definition 5.16 Let $\alpha \in \Re$ and let $\psi$ be a value. Then, the value $\psi$ is $\alpha$ consistent if $\psi_{i}\left(N \backslash U, v^{U, \psi, \alpha}\right)=\psi_{i}(N, v)$ for all $(N, v) \in G$, all nonempty $U \subset N$, and all $i \in N \backslash U$.

Definition 5.17 The value $\psi$ is $\alpha$-standard if for all 2-person games $(\{i, j\}, v), \psi_{i}(\{i, j\}, v)=\frac{v(\{i, j\})-(1-\alpha) v(\{j\})+(1-\alpha) v(\{i\})}{2}$.

It is easily verified that for fixed $\alpha \in \Re$, the value $\psi^{\text {ot }}$ satisfies $\alpha$-standardness. We will now prove $\alpha$-consistency for the $\alpha$-egalitarian Shapley values. The following lemma states that for $\alpha \neq 1$, the $\left(U, \psi^{\alpha}, \alpha\right)$-reduced game is equal to the $(U, \varphi)$-reduced game, i.e., the HM-reduced game for the Shapley value, with only the worth of the grand coalition of this reduced game being changed by an additive term. In the lemma, this is denoted by adding, a multiple of the unanimity game $\left(N \backslash U, u_{N \backslash U}\right)$. The proof follows by writing out the definitions at both sides.

Lemma 5.18 Let $\alpha \in \Re \backslash\{1\}$, let $(N, v) \in G$, and let $U \subset N$ be nonempty. Then $v^{U, \psi^{\alpha}, \alpha}=v^{U, \varphi}+\alpha\left[\sum_{j \in U} \varphi_{j}(N, v)-\frac{|U|}{|N|} v(N)\right] u_{N \backslash U}$.

Using the fact that the Shapley value is 0-consistent, i.e., HM-consistent (cf. Hart \& Mas-Colell [1989]), Lemma 5.18 can be used to show that every value $\psi^{\alpha}$ is $\alpha$-consistent.

Proposition 5.19 For every $\alpha \in \Re$, the value $\psi^{\alpha}$ is $\alpha$-consistent.

Proof. For $\alpha=1$, the statement follows immediately by recalling $\psi^{1}=\eta$, and by noting that 1-consistency is equivalent to HM-consistency. Let $\alpha \in$ 
$\Re \backslash\{1\}$ and let $(N, v)$ be a game and $U \subseteq N$. Let $i \in N \backslash U$, then

$$
\begin{aligned}
& \varphi_{i}\left(N \backslash U, v^{U, \psi^{\alpha}, \alpha}\right) \\
= & \varphi_{i}\left(N \backslash U, v^{U, \varphi}+\alpha\left[\sum_{j \in U} \varphi_{j}(N, v)-\frac{|U|}{|N|} v(N)\right] u_{N \backslash U}\right) \\
= & \varphi_{i}\left(N \backslash U, v^{U, \varphi}\right)+\varphi_{i}\left(N \backslash U, \alpha\left[\sum_{j \in U} \varphi_{j}(N, v)-\frac{|U|}{|N|} v(N)\right] u_{N \backslash U}\right) \\
= & \varphi_{i}(N, v)+\alpha \frac{\left[\sum_{j \in U} \varphi_{j}(N, v)-\frac{|U|}{|N|} v(N)\right]}{|N \backslash U|} .
\end{aligned}
$$

Here, the first equality follows from Lemma 5.18, the second equality from additivity of the Shapley value, and the third equality for the 0-consistency of the Shapley value and the amounts which it assigns in a unanimity game. For the egalitarian value we have:

$$
\begin{aligned}
& \eta_{i}\left(N \backslash U, v^{U, \psi^{\mathrm{a}}, \alpha}\right) \\
= & \eta_{i}\left(N \backslash U, v^{U, \varphi, 0}+\alpha\left[\sum_{j \in U} \varphi_{j}(N, v)-\frac{|U|}{|N|} v(N)\right] u_{N \backslash U}\right) \\
= & \eta_{i}\left(N \backslash U, v^{U, \varphi, 0}\right)+\eta_{i}\left(N \backslash U, \alpha\left[\sum_{j \in U} \varphi_{j}(N, v)-\frac{|U|}{|N|} v(N)\right] u_{N \backslash U}\right) \\
= & \frac{1}{|N \backslash U|}\left[v(N)-\sum_{j \in U} \varphi_{j}(N, v)\right]+\frac{\alpha}{|N \backslash U|}\left[\sum_{j \in U} \varphi_{j}(N, v)-\frac{|U|}{|N|} v(N)\right] \\
= & \eta_{i}(N, v)-(1-\alpha) \frac{\left[\sum_{j \in U} \varphi_{j}(N, v)-\frac{|U|}{|N|} v(N)\right]}{|N \backslash U|} .
\end{aligned}
$$

Again, the first equality follows from Lemma 5.18. The other equalities follow from additivity and the definition. It is now a matter of calculation to find that

$$
\begin{aligned}
\psi_{i}^{\alpha}\left(N \backslash U, v^{U, \psi^{\alpha}, \alpha}\right) & =(1-\alpha) \varphi_{i}\left(N \backslash U, v^{U, \psi^{\alpha}, \alpha}\right)+\alpha \eta_{i}\left(N \backslash U, v^{U, \psi^{\alpha}, \alpha}\right) \\
& =(1-\alpha) \varphi_{i}(N, v)+\alpha \eta_{i}(N, v) \\
& =\psi_{i}^{\alpha}(N, v) .
\end{aligned}
$$

This proves $\alpha$-consistency of $\psi^{\alpha}$. 
We proceed on our way of proving that every $\alpha$-egalitarian Shapley value is characterized by $\alpha$-standardness and $\alpha$-consistency by following the line of proof' in Derks \& Peters [1993]. For this purpose we prove two intermediate results and the main result follows almost immediately from them.

Lemma 5.20 Let $\alpha \in \Re$ and let $\psi$ be an $\alpha$-consistent value such that its restriction to 2-person games is efficient and symmetric. Then $\psi$ is efficient.

Proof. First, let $(\{i\}, v)$ be a 1-person game. We define a 2-person game $(\{i, j\}, w)$ by $w(\{i, j\})=2 v(\{i\}), w(\{i\})=w(\{j\})=v(\{i\})$. By symmetry and efficiency of $\psi$ on 2-person games, $\psi_{j}(\{i, j\}, w)=\psi_{i}(\{i, j\}, w)=$ $v(\{i\})$. Therefore, $w^{\{j\}, \psi, \alpha}(\{i\})=w(\{i, j\})-\psi_{j}(\{i, j\}, w)=2 v(\{i\})-$ $v(\{i\})=v(\{i\})$. Thus, by $\alpha$-consistency of $\psi, \psi_{i}(\{i\}, v)=\psi_{i}\left(\{i\}, w^{\{j\}, \psi, \alpha}\right)=$ $\psi_{i}(\{i, j\}, w)=v(\{i\})$. This shows that the restriction of $\psi$ to 1-person games is efficient.

For the general case, take $(S, v)$ with $|S| \geq 3$ and assume that the restriction of $\psi$ to all games with less than $|S|$ players is efficient. Let $k \in S$, then

$$
\begin{aligned}
\sum_{i \in S} \psi_{i}(S, v) & =\psi_{k}(S, v)+\sum_{i \in S \backslash\{k\}} \psi_{i}(S, v) \\
& =\psi_{k}(S, v)+\sum_{i \in S \backslash\{k\}} \psi_{i}\left(S \backslash\{k\}, v^{\{k\}, \psi, \alpha}\right) \\
& =\psi_{k}(S, v)+v^{\{k\}, \psi, \alpha}(S \backslash\{k\}) \\
& =v(S) .
\end{aligned}
$$

Here, the second equality follows from $\alpha$-consistency of $\psi$, the third equality follows by induction, and the last equality by definition of the $\alpha$-reduced game.

Lemma 5.21 Let $\alpha \in \Re$ and let $\psi$ be an efficient and $\alpha$-consistent value: Let $(N, v)$ be a game with $|N|>2$ and suppose that $\psi(M, w)=\psi^{\alpha}(M, w)$ for all games $(M, w)$ with $|M|<|N|$. Then $\psi(N, v)=\psi^{\alpha}(N, v)$.

Proof. Let $\emptyset \neq M \subset N$ and let $U=N \backslash M$. For $S \subset M$ we have $v^{U, \psi, \alpha}(S)=$ $v^{U, \psi^{\alpha}, \alpha}(S)$. Hence, with the unanimity game $\left(M, u_{M}\right)$, we have

$$
v^{U, \psi, \alpha}=v^{U, \psi^{\alpha}, \alpha}+\beta_{M}(v) u_{M},
$$


where $\beta_{M}(v)=v^{U, \psi, \alpha}(M)-v^{U, \psi^{\alpha}, \alpha}(M)$. Then for all $i \in M$

$$
\begin{aligned}
\psi_{i}^{\alpha}\left(M, v^{U, \psi^{\alpha}, \alpha}\right) & =\psi_{i}^{\alpha}\left(M, v^{U, \psi, \alpha}\right)-\beta_{M}(v) /|M| \\
& =\psi_{i}\left(M, v^{U, \psi, \alpha}\right)-\beta_{M}(v) /|M| .
\end{aligned}
$$

So by $\alpha$-consistency of $\psi^{\alpha}$ and $\psi$, we have for all $i \in M$

$$
\psi_{i}^{\alpha}(N, v)=\psi_{i}(N, v)-\beta_{M}(v) /|M| .
$$

Suppose that $T \subset N$ exists with $\beta_{T}(v) \neq 0$. Then, we have for every $T^{\prime} \subset N$ with $T^{v} \cap T \neq \emptyset$ that $\frac{\beta_{T^{\prime}}(v)}{\left|T^{v}\right|}=\frac{\beta_{T}(v)}{|T|} \neq 0$. Suppose without loss of generality that $\beta_{T}(v)<0$, then by Equation (5.4): $\psi_{i}^{\alpha}(N, v)>\psi_{i}(N, v)$ for all $i \in T$. Now, obviously $N \cap T \neq \emptyset$, hence $\psi_{i}^{\alpha}(N, v)>\psi_{i}(N, v)$ for all $i \in N \backslash T$. So, we have $\psi_{i}^{\alpha}(N, v)>\psi_{i}(N, v)$ for all $i \in N$. This contradicts efficiency of $\psi$ and $\psi^{\alpha}$. Therefore, $\beta_{T}(v)=0$ for every $T \subset N$, so $\psi(N, v)=\psi^{\alpha}(N, v)$ by Equation (5.4).

If the conditions of Lemma 5.21 are met, then by using induction on the number of players, it may be shown that $\psi=\psi^{\alpha}$. Lemmas 5.20 and 5.21 now imply the last axiomatic characterization of an $\alpha$-egalitarian Shapley value in this section.

Proposition 5.22 Let $\alpha \in \Re$ and let $\psi$ be a value. Then, the following two statements are equivalent:

i. $\psi$ is $\alpha$-standard and $\alpha$-consistent;

ii. $\psi=\psi^{\alpha}$.

Proof. Let $\psi$ be a value satisfying $\alpha$-standardness and $\alpha$-consistency. Then, by $\alpha$-standardness $\psi$ is efficient for two-person games, hence $\psi$ is efficient for all games by Lemma 5.20. This implies that $\psi$ fulfills the conditions in Lemma 5.21 for one-person and two-person games, hence $\psi(N, v)=\psi^{\alpha}(N, v)$, for all games $(N, v) \in G$.

\subsection{Potentials, values, and axioms}

In the previous sections we have seen that the (weighted) Shapley value(s) and the egalitarian value are HM-consistent, and that the $\alpha$-egalitarian Shapley values are $\alpha$-consistent. To prove $\alpha$-consistency of the latter family, we 
used an indirect approach using the consistency of the Shapley value. This absence of a direct proof inspired our investigations for this section. In Hart \& Mas-Colell [1989] an important tool for the proof of HM-consistency of the $w$-weighted Shapley values was introduced, namely the $w$-potentials. In this section, we introduce a family of potentials generalizing the potentials of Hart \& Mas-Colell [1989], and associate with each potential a unique efficient and linear value. The families of potentials and values to be introduced depend on a tuple of parameters $(a, b, \alpha)$. The vectors $a, b \in \Re^{Z}$ are exogenously given weights similar to the weights of the weighted Shapley values. As before, the parameter $\alpha \in \Re$ is a measure of the level of egalitarianism of the society.

We show that for $\alpha=0$ or $\alpha=1$, all values within this family satisfy HMconsistency. To obtain the latter result, we make use of the corresponding $(a, b, \alpha)$-potential. The proof of this result is direct. For $\alpha \notin\{0,1\}$, we have that $\alpha$-consistency is fulfilled if and only if the value satisfies equal coalitional improvement. To obtain this result we use our earlier result of HM-consistency for $\alpha=0$. We also list restrictions on the parameters $(a, b, \alpha)$ and their effects on other properties of the values $\Phi^{a, b, \alpha}$.

We close the section by showing that certain (sub-)families of values $\Phi^{a, b, \alpha}$ are uniquely determined by some form of consistency and the amounts attributed in two-person, similar to the characterization of Hart \& Mas-Colell [1989] of the weighted Shapley values and our characterization of the $\alpha$ egalitarian Shapley values in the preceding section.

Definition 5.23 Let $a, b \in \Re^{Z}, \alpha \in \Re$, satisfying $\sum_{i \in S} a_{i} \neq 0$ for all nonempty $S \subseteq Z$ and $b_{i} \neq 0$ for all $i \in Z$. Then the $(\mathbf{a}, \mathbf{b}, \alpha)$-potential is the unique map $P^{a, b, \alpha}: G \rightarrow \Re$ given by:

i. $P^{a, b, \alpha}(\emptyset, v)=0$,

ii. $\sum_{i \in N}\left[a_{i} P^{a, b, \alpha}(N, v)-b_{i} P^{\alpha, b, \alpha}(N \backslash\{i\}, v)\right]=(1-\alpha) v(N)$, for all $(N, v) \in G, N \neq \emptyset$.

The value $\Phi^{\mathrm{a}, \mathrm{b}, \alpha}$ is given by

$$
\Phi_{i}^{a, b, \alpha}(N, v)=a_{i} P^{a, b, \alpha}(N, v)-b_{i} P^{a, b, \alpha}(N \backslash\{i\}, v)+\alpha \frac{v(N)}{|N|}
$$

for all $(N, v) \in G, i \in N$. 
Remark 4 We only consider parameters $(a, b, \alpha)$ which satisfy $\sum_{i \in S} a_{i} \neq 0$ for all $S \subseteq Z$ and $b_{i} \neq 0$ for all $i \in Z$. Then, Definition 5.23 implies $P^{a, b, a}(N, v)=\frac{(1-a) v(N)+\sum_{k \in N^{b}} b_{k} P^{a, b, a}(N \backslash\{k\}, v)}{\sum_{k \in N} a_{k}}$, which can be used to determine both the $(a, b, \alpha)$-potential and the connected value recursively.

Remark 5 Definition 5.23 allows a unified representation of the (weighted) Shapley value(s) and the $\alpha$-egalitarian Shapley values:

- If $a_{i}=b_{i}>0$ for all $i \in Z$ and $\alpha=0$, then $P^{a, b, \alpha}=P_{w}$ and $\Phi^{a, b, a}=\varphi^{w}$ where $w=a=b$;

- If $a_{i}=b_{i}=1$ for all $i \in Z$ and $\alpha \in \Re$, then $\Phi^{a, b, a}=\psi^{\alpha}$.

An interpretation of the value $\Phi^{a, b, \alpha}$ is that for an arbitrary game $(N, v)$ it gives to player $i \in N$ the sum of the proportion $\alpha$ of the per-capita income of the grand coalition, and his linearly modified marginal contribution to the potential of the taxed game $v^{\alpha}$. The following lemma may be proven by induction and by using Definition 5.23.

Lemma $5.24 \Phi^{a, b, \alpha}$ is efficient, linear, trivial, and homogeneous of degree 0 in $(a, b)$.

The following three propositions show connections between restrictions on the parameters $(a, b, \alpha)$ and several concepts presented in the previous sections.

Proposition $5.25 \Phi^{a, b, 1}$ is symmetric. For $\alpha \neq 1$, the following statements are equivalent:

i. $\Phi^{a, b, a}$ is symmetric;

ii. There exist $\tau_{1}, \tau_{2} \in \Re \backslash\{0\}$ such that $a_{i}=\tau_{1}, b_{i}=\tau_{2}$ for all $i \in Z$;

iii. For all games $(N, v), i \in N$, it holds that

$$
\begin{aligned}
& P^{a, b, \alpha}(N, v)=(1-\alpha) \sum_{\emptyset \neq S \subseteq N} \frac{(|S|-1) !(|N|-|S|) !}{|N| !}\left(\frac{\tau_{2}}{\tau_{1}}\right)^{|N|-|S|} \frac{v(S)}{\tau_{1}} ; \\
& \Phi_{i}^{a, b, \alpha}(N, v)=\alpha \frac{v(N)}{|N|}+ \\
& (1-\alpha) \sum_{\emptyset \neq S \subseteq N \backslash\{i\}} \frac{|S| \cdot|N|-|S|-1) !}{|N| !}\left(\frac{\tau_{2}}{\tau_{1}}\right)^{|N|-|S|-1}\left[v(S \cup\{i\})-\left(\frac{\tau_{2}}{\tau_{1}}\right) v(S)\right] .
\end{aligned}
$$

Proof. See Section 5.5. 
Proposition $5.26 \Phi^{a, b, 1}$ satisfies 1-egalitarianism and 1-marginality. For $\alpha \neq 1$, the following statements are equivalent:

i. $\Phi^{a, b, \alpha}$ satisfies $\alpha$-egalitarianism;

ii. $\Phi^{a, b, \alpha}$ satisfies $\alpha$-marginality;

iii. $a=b$.

Proof. See Section 5.5.

Proposition $5.27 \Phi^{a, b, 1}$ satisfies equal coalitional improvement. For $\alpha \neq 1$, the following statements are equivalent:

i. $\Phi^{a, b, \alpha}$ satisfies equal coalitional improvement;

ii. There exists $\tau \in \Re \backslash\{0\}$ satisfying $a_{i}=\tau$ for all $i \in Z$.

Proof. See Section 5.5.

We will now use $(a, b, 0)$-potentials to prove HM-consistency of the corresponding value $\Phi^{a, b, 0}$.

Proposition 5.28 For $\alpha=0$ or $\alpha=1, \Phi^{a, b, \alpha}$ is HM-consistent.

Proof. See Section 5.5.

So for all $a, b \in \Re^{Z}$ we have HM-consistency whenever $\alpha=0$ or $\alpha=1$. This shows that the weighted Shapley values form. only a subclass of the HM-consistent values. We now prove equivalence of $\alpha$-consistency and equal coalitional improvement for the values $\Phi^{a, b, \alpha}$ for $\alpha \notin\{0,1\}$.

Proposition 5.29 For $\alpha \notin\{0,1\}$ the following statements are equivalent:

i. $\Phi^{a, b, \alpha}$ satisfies equal coalitional improvement;

ii. $\Phi^{a, b, \alpha}$ is $\alpha$-consistent.

Proof. See Section 5.5.

We conclude this section by describing two families of values. We characterize each value in the first family by HM-consistency and $\alpha$-standardness, and each value in the second family by $\alpha$-consistency and standardness. 
Definition 5.30 Let $\gamma \in \Re$. Then, the value $\xi^{\gamma}$ is for every game $(N, v)$ and $i \in N$ given by

$\xi_{i}^{\gamma}(N, v)=\sum_{0 \neq S \subseteq N \backslash\{i\}} \frac{|S| !(|N|-|S|-1) !}{|N| !}(1-\gamma)^{|N|-|S|-1}[v(S \cup\{i\})-(1-\gamma) v(S)]$.

Definition 5.31 Let $\alpha \in \Re, \gamma \in \Re \backslash\{0\}$. Then, the value $\omega^{\gamma, \alpha}$ is for every game $(N, v)$ and $i \in N$ given by

$\omega_{i}^{\gamma, \alpha}(N, v)=\alpha \frac{v(N)}{|N|}+$

$(1-\alpha) \sum_{0 \neq S \subseteq N \backslash\{i\}} \frac{|S| \cdot(|N|-|S|-1) !}{|N| !}\left(\frac{1}{1-\gamma}\right)^{|N|-|S|-1}\left[v(S \cup\{i\})-\frac{1}{1-\gamma} v(S)\right]$.

Proposition 5.32 The following two statements are equivalent:

i. The value $\psi$ is HM-consistent and $\alpha$-standard;

ii. $\psi=\xi^{\circ}$.

Proof. First we give an alternative representation of $\xi^{a}$. Take $(a, b, 0)$ with $a_{i}=1, b_{i}=1-\alpha$ for all $i \in N$, then $\Phi^{a, b, 0}=\xi^{\alpha}$ (cf. Proposition 5.25). By Proposition 5.28, $\xi^{\alpha}$ is HM-consistent. Furthermore, $\alpha$-standardness follows immediately by writing out Definition 5.30 for two-person games. So the part that (ii) implies (i) is clear.

We now prove that (i) implies (ii). Note that $\psi$ is efficient and symmetric for two-person games by $\alpha$-standardness. Hence, $\psi$ satisfies efficiency for all games by Lemma 5.20. So, $\psi=\xi^{\alpha}$ for all one- and two-person games, $\psi$ is efficient and 0 -consistent, hence the conditions of Lemma 5.21 are met. This implies that $\psi=\xi^{\alpha}$.

Proposition 5.33 The following two statements are equivalent for $\alpha \neq 1$ :

i. The value $\psi$ is $\alpha$-consistent and standard;

ii. $\psi=\omega^{\alpha, \alpha}$.

Proof. Let $\alpha \neq 1$. First we give an alternative representation of $\omega^{\alpha, \alpha}$. Take $(a, b, \alpha)$ with $a_{i}=1, b_{i}=\frac{1}{1-\alpha}$ for all $i \in N$, then $\Phi^{a, b, \alpha}=\omega^{\alpha, \alpha}$. By Proposition $5.25 \omega^{\alpha, \alpha}$ is symmetric, hence $\omega^{\alpha, \alpha}$ satisfies equal coalitional improvement, which by Proposition 5.29 implies that it satisfies $\alpha$-consistency. Standardness follows immediately by writing out Definition 5.31 for two-person games. So the part that (ii) implies (i) should be clear. 
We now prove that (i) implies (ii). By standardness $\omega^{\alpha, \alpha}$ is efficient and symmetric with respect to two-person games, therefore this value is efficient for all games by Lemma 5.20. So, $\psi=\omega^{\alpha, \alpha}$ for all one- and two-person games, $\psi$ is efficient and $\alpha$-consistent, hence the conditions of Lemma 5.21 are met. This implies that $\psi=\omega^{\alpha, \alpha}$.

\subsection{Appendix}

Proof of Proposition 5.25. We only prove the part for $\alpha \neq 1$.

(ii) implies (i): Take $k, l \in Z$ and $(N, v)$ with $k, l \in N$ symmetric players. We prove for $(a, b, \alpha)$ with $a_{i}=\tau_{1}$ and $b_{i}=\tau_{2}$ for all $i \in Z$ that $P^{a, b, \alpha}(N \backslash\{k\}, v)=P^{a, b, \alpha}(N \backslash\{l\}, v)$ by induction on the cardinality of $S \subseteq$ $N \backslash\{k, l\}$.

For $S=\emptyset$, we obtain $P^{a, b, \alpha}(S \cup\{k\}, v)-P^{a, b, \alpha}(S \cup\{l\}, v)=\frac{(1-\alpha) v(\{k\})}{\tau_{1}}-$ $\frac{(1-\alpha) v(\{l\})}{\tau_{1}}=0$. Now, let $S \subseteq N \backslash\{k, l\}$ and assume for all $D \subset S: P^{a, b, \alpha}(D \cup$ $\{k\}, v)=P^{a, b, \alpha}(D \cup\{l\}, v)$. Then,

$$
\begin{aligned}
& P^{a, b, \alpha}(S \cup\{k\}, v)-P^{a, b, \alpha}(S \cup\{l\}, v) \\
= & \frac{(1-\alpha) v(S \cup\{k\})+\sum_{i \in S \cup\{k\}} \tau_{2} P^{a, b, \alpha}(S \cup\{k\} \backslash\{i\})}{\sum_{i \in S \cup\{k\}} \tau_{1}}- \\
& \frac{(1-\alpha) v(S \cup\{l\})+\sum_{i \in S \cup\{l\}} \tau_{2} P^{a, b, \alpha}(S \cup\{l\} \backslash\{i\})}{\sum_{i \in S \cup\{l\}} \tau_{1}} \\
= & 0 .
\end{aligned}
$$

This in turn implies:

$$
\begin{aligned}
\Phi_{k}^{a, b, \alpha}(N, v) & =\tau_{1} P^{a, b, \alpha}(N, v)-\tau_{2} P^{a, b, \alpha}(N \backslash\{k\}, v)+\alpha \frac{v(N)}{|N|} \\
& =\tau_{1} P^{a, b, \alpha}(N, v)-\tau_{2} P^{a, b, \alpha}(N \backslash\{l\}, v)+\alpha \frac{v(N)}{|N|} \\
& =\Phi_{l}^{a, b, \alpha}(N, v) .
\end{aligned}
$$

(i) implies (ii): Take $i, j \in Z$, then by symmetry of $\Phi^{a, b, \alpha}$

$$
\begin{aligned}
0 & =\Phi_{i}^{a, b, a}\left(\{i, j\}, u_{\{i, j\}}\right)-\Phi_{j}^{a, b, \alpha}\left(\{i, j\}, u_{\{i, j\}}\right) \\
& =a_{i} P^{a, b, a}\left(\{i, j\}, u_{\{i, j\}}\right)-b_{i} P^{a, b, a}\left(\{j\}, u_{\{i, j\}}\right)+\alpha \frac{u_{\{i, j\}}(\{i, j\})}{2}-
\end{aligned}
$$




$$
\begin{aligned}
& {\left[a_{j} P^{a, b, \alpha}\left(\{i, j\}, u_{\{i, j\}}\right)-b_{j} P^{a, b, a}\left(\{i\}, u_{\{i, j\}}\right)+\alpha \frac{u_{\{i, j\}}(\{i, j\})}{2}\right] } \\
= & \left(a_{i}-a_{j}\right) P^{a, b, \alpha}\left(\{i, j\}, u_{\{i, j\}}\right) .
\end{aligned}
$$

Since $P^{a, b, a}\left(\{i, j\}, u_{\{i, j\}}\right) \neq 0$, we obtain $a_{i}=a_{j}$. So take $\tau_{1}$ such that $\tau_{1}=a_{i}$ for all $i \in Z$.

Take $(\{i, j\}, v)$ with $v(\{i\})=v(\{j\}) \neq 0$. Then, by symmetry of $\Phi^{a, b, \alpha}$

$$
\begin{aligned}
0= & \Phi_{i}^{a, b, a}(\{i, j\}, v)-\Phi_{j}^{a, b, \alpha}(\{i, j\}, v) \\
= & \tau_{1} P^{a, b, \alpha}(\{i, j\}, v)-b_{i} P^{a, b, a}(\{j\}, v)+\alpha \frac{v(\{i, j\})}{2}- \\
& {\left[\tau_{1} P^{a, b, \alpha}(\{i, j\}, v)-b_{j} P^{a, b, \alpha}(\{i\}, v)+\alpha \frac{v(\{i, j\})}{2}\right] } \\
= & -b_{i} P^{a, b, \alpha}(\{j\}, v)+b_{j} P^{a, b, a}(\{i\}, v) \\
= & -b_{i} \frac{(1-\alpha) v(\{j\})}{\tau_{1}}+b_{j} \frac{(1-\alpha) v(\{i\})}{\tau_{1}} \\
= & \left(b_{j}-b_{i}\right) \frac{(1-\alpha)}{\tau_{1}} v(\{i\}) .
\end{aligned}
$$

So take $\tau_{2}$ such that $b_{i}=\tau_{2}$ for all $i \in Z$.

(iii) implies (i): Straightforward. (ii) implies (iii): Follows by induction and writing out Def. 5.23 for $\tau_{1}, \tau_{2}$.

Proof of Proposition 5.26. We only prove the part for $\alpha \neq 1$.

(iii) implies (i): We first prove $P^{a, a, \alpha}(N, v)=P^{a, a, \alpha}(N \backslash\{i\}, v)$ whenever $i \in \mathcal{N}(N, v)$, by induction on $|N|$.

If $N=\{i\}$, the statement holds trivially. Now, let $|N| \geq 2$, and assume for all games $(S, v)$ with $|S|<|N|$ that $P^{a, a, \alpha}(S, v)=P^{a, a, \alpha}(S \backslash\{i\}, v)$ whenever $i \in \mathcal{N}(S, v)$. Then, for $i \in \mathcal{N}(N, v)$,

$$
\begin{aligned}
& P^{a, a, \alpha}(N, v) \\
= & \frac{(1-\alpha) v(N)+\sum_{k \in N} a_{k} P^{a, a, \alpha}(N \backslash\{k\}, v)}{\sum_{k \in N} a_{k}} \\
= & \frac{(1-\alpha) v(N \backslash\{i\})+\sum_{k \in N \backslash\{i\}} a_{k} P^{a, a, \alpha}(N \backslash\{i, k\}, v)+a_{i} P^{a, a, \alpha}(N \backslash\{i\}, v)}{\sum_{k \in N} a_{k}} \\
= & \frac{\sum_{k \in N \backslash\{i\}} a_{k} P^{a, a, \alpha}(N \backslash\{i\}, v)+a_{i} P^{a, a, \alpha}(N \backslash\{i\}, v)}{\sum_{k \in N} a_{k}} \\
= & P^{a, a, \alpha}(N \backslash\{i\}, v) .
\end{aligned}
$$


The second equality follows from $v(N)=v(N \backslash\{i\})$ and the induction assumption.

This in turn implies: $\Phi_{i}^{a, a, \alpha}(N, v)=a_{i} P^{a, a, \alpha}(N, v)-a_{i} P^{a, a, \alpha}(N, v)+\alpha \frac{v(N)}{|N|}=$ $\alpha \frac{v(N)}{|N|}$ whenever $i \in \mathcal{N}(N, v)$.

(i) implies (iii): Let $i, j \in Z$, obviously $\alpha$-egalitarianism of $\Phi^{a, b, \alpha}$ implies $a_{i} P^{a, b, \alpha}\left(\{i, j\},, u_{\{j\}}\right)-b_{i} P^{a, b, \alpha}\left(\{j\}, u_{\{j\}}\right)=0$. Therefore

$$
\begin{aligned}
0 & =a_{i} P^{a, b, \alpha}\left(\{i, j\}, u_{\{j\}}\right)-b_{i} P^{a, b, \alpha}\left(\{j\}, u_{\{j\}}\right) \\
& =(1-\alpha)\left[a_{i} \frac{1+b_{i} \frac{1}{a_{j}}}{a_{i}+a_{j}}-b_{i} \frac{1}{a_{j}}\right] \\
& =(1-\alpha)\left[\frac{a_{i}+b_{i} \frac{a_{i}}{a_{j}}-b_{i} \frac{a_{i}}{a_{j}}-b_{i} \frac{a_{j}}{a_{j}}}{a_{i}+a_{j}}\right] \\
& =(1-\alpha)\left[\frac{a_{i}-b_{i}}{a_{i}+a_{j}}\right] .
\end{aligned}
$$

Hence, $a_{i}=b_{i}$. This in turn implies $a=b$.

(iii) implies (ii): Take $i \in Z$. Take $(N, w)$ and $(N, \widetilde{w})$ with $i \in N$ and $\Delta_{i}^{w}(S)=\Delta_{i}^{\tilde{w}}(S)$. Observe that $i \in \mathcal{N}(N, \tilde{w}-w)$, and since (iii) implies (i), we get. $\Phi^{a, b, \alpha}(N, \tilde{w}-w)=\frac{\alpha(\tilde{w}-w)(N)}{|N|}=\alpha \overline{\Phi^{a, b, \alpha}(N, \widetilde{w})}-\alpha \overline{\Phi^{a, b, \alpha}(N, w)}$. By linearity of $\Phi^{a, b, \alpha}$ we have

$$
\begin{aligned}
\Phi_{i}^{a, b, \alpha}(N, \widetilde{w}) & =\Phi_{i}^{a, b, \alpha}(N, \tilde{w}-w+w) \\
& =\Phi_{i}^{a, b, \alpha}(N, \widetilde{w}-w)+\Phi_{i}^{a, b, \alpha}(N, w) \\
& =\Phi_{i}^{a, b, \alpha}(N, w)-\alpha \overline{\Phi^{a, b, \alpha}(N, w)}+\alpha \overline{\Phi^{a, b, \alpha}(N, \tilde{w})} .
\end{aligned}
$$

This proves $\alpha$-marginality of $\Phi^{a, b, \alpha}$.

(ii) implies (iii): Take $i \in Z, N \ni i,\left(N, v_{0}\right)$, and $\left(N, u_{N \backslash\{i\}}\right)$. Then, by $\alpha$-marginality

$$
\Phi_{i}^{a, b, \alpha}\left(N, v_{0}\right)-\alpha \overline{\Phi^{a, b, \alpha}\left(N, v_{0}\right)}=\Phi_{i}^{a, b, \alpha}\left(N, u_{N \backslash\{i\}}\right)-\alpha \overline{\Phi^{a, b, \alpha}\left(N, u_{N \backslash\{i\}}\right)} .
$$

Hence, by efficiency and triviality, we get $\Phi_{i}^{a, b, \alpha}\left(N, u_{N \backslash\{i\}}\right)=\frac{a}{|N|}$. This implies in turn that $a_{i} P^{a, b, \alpha}\left(N, u_{N \backslash\{i\}}\right)-b_{i} P^{a, b, \alpha}\left(N \backslash\{i\}, u_{N \backslash\{i\}}\right)=0$, which leads to

$$
0=a_{i} P^{a, b, \alpha}\left(N, u_{N \backslash\{i\}}\right)-b_{i} P^{a, b, \alpha}\left(N \backslash\{i\}, u_{N \backslash\{i\}}\right)
$$




$$
\begin{aligned}
& =(1-\alpha)\left[a_{i} \frac{1+b_{i} \sum_{k \in N \backslash(0\}} a_{k}}{\sum_{k \in N} a_{k}}-b_{i} \frac{1}{\sum_{k \in N \backslash\{i\}} a_{k}}\right] \\
& =(1-\alpha) \frac{a_{i} \sum_{k \in N \backslash\{i\}} a_{k}+a_{i} b_{i}-b_{i} \sum_{k \in N} a_{k}}{\sum_{k \in N} a_{k}\left(\sum_{k \in N \backslash\{i\}} a_{k}\right)} \\
& =(1-\alpha) \frac{a_{i}-b_{i}}{\sum_{k \in N} a_{k}} \text {. }
\end{aligned}
$$

Hence, $a_{i}=b_{i}$. This proves $a=b$.

Proof of Proposition 5.27. We only prove that (i) implies (ii). Observe that for all $(N, v), T \subseteq N, P^{a, b, a}\left(N \backslash\{k\}, c u_{T}\right)=0$ for all $k \in T$, as $\left(N \backslash\{k\}, c u_{T}\right)$ is a zero-game. So we obtain $\Phi_{k}^{a, b, \alpha}\left(N, c u_{T}\right)=a_{k} P^{a, b, \alpha}\left(N, c u_{T}\right)+$ $\frac{\alpha c}{|N|}$. Let $i, j \in Z$, clearly $\Phi_{i}^{a, b, \alpha}\left(\{i, j\}, v_{0}\right)=\Phi_{j}^{a, b, \alpha}\left(\{i, j\}, v_{0}\right)=0$, and $\left(\{i, j\}, u_{\{i, j\}}\right)=$ $\left(\{i, j\}, v_{0}+u_{\{i, j\}}\right)$. By equal coalitional improvement.

$$
\begin{aligned}
& \Phi_{i}^{a, b, \alpha}\left(\{i, j\}, u_{\{i, j\}}\right)-\Phi_{j}^{a, b, \alpha}\left(\{i, j\}, u_{\{i, j\}}\right) \\
= & \Phi_{i}^{a, b, \alpha}\left(\{i, j\}, v_{0}+u_{\{i, j\}}\right)-\Phi_{j}^{a, b, \alpha}\left(\{i, j\}, v_{0}+u_{\{i, j\}}\right) \\
= & \Phi_{i}^{a, b, \alpha}\left(\{i, j\}, v_{0}\right)+c-\left[\Phi_{j}^{a, b, \alpha}\left(\{i, j\}, v_{0}\right)+c\right] \\
= & 0 .
\end{aligned}
$$

So, $a_{i} P^{a, b, \alpha}\left(\{i, j\}, u_{\{i, j\}}\right)+\frac{\alpha}{|N|}=a_{j} P^{a, b, \alpha}\left(\{i, j\}, u_{\{i, j\}}\right)+\frac{a}{|N|}$ which proves $a_{i}=a_{j}$.

Proof of Proposition 5.28. For $\alpha=1$, HM-consistency follows immediately by noting $\Phi^{a, b, \alpha}=\eta$.

We now prove the part for $\alpha=0$. Let $(N, v) \in G$. For $U \subset N$ satisfying $|U|=|N|-1$, the proposition follows immediately. Now fix $U \subset N$ satisfying $0 \neq|U|<|N|-1$. To prove the proposition for $\alpha=0$ we show

$$
P^{a, b, 0}(N, v)=P^{a, b, 0}\left(N \backslash U, v^{U, \Phi^{a, b, 0}}\right)+\prod_{k \in N \backslash U} \frac{b_{k}}{a_{k}} P^{a, b, 0}(U, v) .
$$

We do this by induction on the cardinality of nonempty subsets $S$ of $N \backslash U$. Note that for all $N \subseteq Z$, and $\emptyset \neq U \subset N$, we have

$$
P^{a, b, 0}(N, v)=\frac{v(N)-\sum_{k \in U} \Phi_{k}^{a, b, 0}(N, v)+\sum_{k \in N \backslash U} b_{k} P^{a, b, 0}(N \backslash\{k\}, v)}{\sum_{k \in N \backslash U} a_{k}} .
$$


Hence, taking $i \in N \backslash U$, we have

$$
\begin{aligned}
P^{a, b, 0}(\{i\} \cup U, v) & =\frac{v(\{i\} \cup U)-\sum_{k \in U} \Phi_{k}^{a, b, 0}(\{i\} \cup U, v)+b_{i,} P^{a, b, 0}(U, v)}{a_{i}} \\
& =\frac{v^{U, \Phi^{a, b, 0}}(\{i\})}{a_{i}}+\frac{b_{i}}{a_{i}} P^{a, b, 0}(U, v) \\
& =P^{a, b, 0}\left(\{i\}, v^{U, \Phi^{a, b, 0}}\right)+\frac{b_{i}}{a_{i}} P^{a, b, 0}(U, v) .
\end{aligned}
$$

Let $S \subseteq N \backslash U,|S| \geq 2$, and assume for all $D \subset S$ :

$$
P^{a, b, 0}(D \cup U, v)=P^{a, b, 0}\left(D, v^{U, \Phi^{a, b, 0}}\right)+\prod_{i \in D} \frac{b_{i}}{a_{i}} P^{a, b, 0}(U, v) .
$$

Then,

$$
\begin{aligned}
& P^{a, b, 0}(S \cup U, v) \\
= & \frac{v\{S \cup U)-\sum_{k \in U} \Phi^{a, b, 0}(S \cup U, v)+\sum_{k \in S} b_{k} P^{a, b, 0}((S \cup U) \backslash\{k\}, v)}{\sum_{k \in S} a_{k}} \\
= & \frac{v^{U, \Phi^{a, b, 0}}(S)+\sum_{k \in S} b_{k} P^{a, b, 0}((S \cup U) \backslash\{k\}, v)}{\sum_{k \in S} a_{k}} \\
= & \frac{v^{U, \Phi^{a, b, 0}}(S)+\sum_{k \in S} b_{k} P^{a, b, 0}\left(S \backslash\{k\}, v^{U, \Phi^{a, b, 0}}\right)}{\sum_{k \in S} a_{k}}+ \\
= & \frac{\sum_{k \in S} b_{k}\left[\prod_{i \in S \backslash\{k\}} \frac{b_{i}}{a_{i}} P^{a, b, 0}(U, v)\right]}{\sum_{k \in S} a_{k}} \\
= & P^{a, b, b}\left(S, v^{U, \Phi^{a, b, 0}}\right)+\frac{\sum_{k \in S} b_{k}\left[\prod_{i \in S \backslash\{k\}} \frac{b_{i}}{a_{i}} P^{a, b, 0}(U, v)\right]}{\sum_{k \in S} a_{k}} \\
= & P^{a, b, 0}\left(S, v^{U, \Phi^{a, b, 0}}\right)+\frac{\sum_{k \in S} a_{k}\left[\prod_{i \in S} \frac{b_{i}}{a_{i}} P^{a, b, b}(U, v)\right]}{\sum_{k \in S} a_{k}} \\
& \prod_{i \in S} \frac{b_{i}}{a_{i}} P^{a, b, 0}(U, v) .
\end{aligned}
$$

The second equality follows from Definition 5.4, the third equality follows from Equation (5.6).

Now, let $k \in N \backslash U$, then by definition of $\Phi^{a, b, 0}$ applied to the game $(N, v)$

$$
\Phi_{k}^{a, b, 0}(N, v)
$$




$$
\begin{aligned}
= & a_{k} P^{a, b, 0}(N, v)-b_{k} P^{a, b, 0}(N \backslash\{k\}, v) \\
= & a_{k} P^{a, b, 0}\left(N \backslash U, v^{U, \Phi^{a, b, 0}}\right)-b_{k} P^{a, b, 0}\left(N \backslash(U \cup\{k\}), v^{U, \Phi^{a, b, 0}}\right)+ \\
& a_{k}\left[\prod_{i \in N \backslash U} \frac{b_{i}}{a_{i}} P^{a, b, 0}(U, v)\right]-b_{k}\left[\prod_{i \in N \backslash(U \cup\{k\})} \frac{b_{i}}{a_{i}} P^{a, b, 0}(U, v)\right] \\
= & \Phi_{k}^{a, b, 0}\left(N \backslash U, v^{U, \Phi^{a, b, 0}}\right)+\left[\frac{\prod_{i \in N \backslash U} b_{i}}{\prod_{i \in N \backslash(U \cup\{k\})} a_{i}}-\frac{\prod_{i \in N \backslash U} b_{i}}{\prod_{i \in N \backslash(U \cup\{k\})} a_{i}}\right] P^{a, b, 0}(U, v) . \\
= & \Phi_{k}^{a, b, 0}\left(N \backslash U, v^{U, \Phi^{a, b, 0}}\right) .
\end{aligned}
$$

The second equality follows from Equation (5.5), the third equality follows by definition of the value $\Phi^{a, b, 0}$ applied to the game $\left(N \backslash U, v^{U, \Phi^{a, b, 0}}\right)$.

Proof of Proposition 5.29. We prove the proposition along the lines of the proof of Proposition 5.19. Observe in this context that for $(N, v) \in G$ and nonempty $U \subseteq N$,

$$
v^{U, \Phi^{a, b, a}, \alpha}=v^{U, \Phi^{a, b, 0}}+\alpha\left[\sum_{k \in U} \Phi_{k}^{a, b, 0}(N, v)-\frac{|U|}{|N|} v(N)\right] u_{N \backslash U} .
$$

Note the similarity of the latter with Lemma 5.18. Let $\alpha \notin\{0,1\}$ and $\emptyset \neq U \subset N$, then for $i \in N \backslash U$

$$
\begin{aligned}
& \Phi_{i}^{a, b, 0}\left(N \backslash U, v^{U, \Phi^{a, b, 0}, \alpha}\right) \\
= & \Phi_{i}^{a, b, 0}\left(N \backslash U, v^{U, \Phi^{a, b, 0}}+\alpha\left[\sum_{k \in U} \Phi_{k}^{a, b, 0}(N, v)-\frac{|U|}{|N|} v(N)\right] u_{N \backslash U}\right) \\
= & \Phi_{i}^{a, b, 0}\left(N \backslash U, v^{U, \Phi^{a, b, 0}}\right)+\alpha\left[\sum_{k \in U} \Phi_{k}^{a, b, 0}(N, v)-\frac{|U|}{|N|} v(N)\right] \Phi_{i}^{a, b, 0}\left(N \backslash U, u_{N \backslash U}\right) \\
= & \Phi_{i}^{a, b, 0}(N, v)+\alpha\left[\sum_{k \in U} \Phi_{k}^{a, b, 0}(N, v)-\frac{|U|}{|N|} v(N)\right] \Phi_{i}^{a, b, 0}\left(N \backslash U, u_{N \backslash U}\right) .
\end{aligned}
$$

The second equality follows from Lemma 5.24 and the third by consistency of $\Phi^{a, b, 0}$. Furthermore,

$$
\eta_{i}\left(N \backslash U, v^{U, \Phi^{a, b, a}, \alpha}\right)=\eta_{i}(N, v)-(1-\alpha) \frac{\left[\sum_{k \in U} \Phi_{k}^{a, b, 0}(N, v)-\frac{|U|}{N \mid} v(N)\right]}{|N \backslash U|} .
$$


It is now a matter of calculation to find that $\Phi_{i}^{a, b, \alpha}\left(N \backslash U, v^{U, \Phi^{a, b, \alpha}, \alpha}\right)=$ $\Phi_{i}^{a, b, \alpha}(N, v)$ if and only if $\Phi_{i}^{a, b, 0}\left(N \backslash U, u_{N \backslash U}\right)=\frac{1}{|N \backslash U|}$. The latter holds if and only if $\Phi^{a, b, 0}$ (and hence $\Phi^{a, b, \alpha}$ ) satisfies equal coalitional improvement.

\subsection{References}

Chun, Y., 1989, A new axiomatization of the Shapley value, Games and Economic Behavior 1, 119-130.

Derks, J., \& H. Peters, 1993, Consistent, restricted Shapley values, in A. Bachem, U. Derigs, M. Jünger, R. Schrader (eds.), Physica-Verlag, Heidelberg, pp. 100-103.

Dutta, B., \& D. Ray, 1989, A concept of egalitarianism under participation constraints, Econometrica 57, 615-635.

Hart, S., \& A. Mas-Colell, 1989, Potential, value and consistency, Econometrica 57, 589-614.

Kalai, E., \& D. Samet, 1987, On weighted Shapley values, International Journal of Game Theory 16, 205-222.

Nowak, A.S., \& 'T. Radzik, 1994, A solidarity value for $n$-person transferable utility games, International Journal of Game Theory 23, 43-48.

Roth, A.E., 1988, "The Shapley value: Essays in honor of Lloyd S. Shapley", Cambridge University Press, Cambridge.

Schmeidler, D., 1969, The mucleolus of a characteristic function game, SIAM Journal of Applied Mathematics 17, 1163-1170.

Shapley, L.S., 1953, A value for $n$-person games, in H.W. Kuhn, A.W. Theker (eds.), "Contributions to the Theory of Games II", Princeton University Press, Princeton, pp. 307-317. 
Tijs, S.H., 1981, Bounds for the core and the $\tau$-value, in O. Moeschlin and D. Pallaschke (eds.), "Game Theory and Mathematical Economics", NorthHolland, Amsterdam, pp. 123-132.

Young, H.P., 1985, Monotonic solutions of cooperative games, International Journal of Game Theory 14, 65-72. 



\section{Chapter 6}

\section{Addenda}

\subsection{Samenvatting (Summary in Dutch)}

Dit proefschrift bevat resultaten aangaande dynamische processen, en een onderwerp in de coöperatieve speltheorie. De dynamische processen vinden hun oorsprong in modellen uit de wiskundige economie, de (evolutionaire) speltheorie en de evolutionaire economie. Het inhoudelijke gedeelte van dit. proefschrift bestaat uit vier hoofdstukken die los van elkaar gelezen kunnen worden. Elk hoofdstuk heeft een inleiding, een deel waarin het model beschreven wordt, een of meerdere delen waarin resultaten gepresenteerd worden en een literatuurlijst. We beschrijven hier kort de inhoud van de Hoofdstukken 2 tot en met 5, en verwijzen voor meer gedetaileerde beschrijvingen naar de inleidingen van de hoofdstukken zelf.

Hoofdstuk 2, 'Economic adjustment processes', behandelt economische aanpassingsprocessen in de wiskundige economie. We spreken van een economisch evenwicht indien op alle markten in de economie het aanbod van de goederen precies gelijk is aan de vraag naar deze goederen. Van eminent belang in dit verband zijn de prijzen van goederen. Op deze prijzen richt de analyse zich voornamelijk in de context van een zogenaamde ruileconomie. Een evenwichtsprijs is een vector van prijzen yoor alle goederen in een ruileconomie, waarbij een economisch evenwicht optreedt. Drie vragen hebben traditioneel de aandacht getrokken in de vakliteratuur. Bestaat er een evenwichtsprijs voor elke ruileconomie? Kan een evenwichtsprijs bereikt worden door middel van geleidelijke aanpassingen, te beginnen met een prijs die geen evenwichtsprijs is? Kan een evenwichtsprijs berekend of benaderd 
worden? Ten eerste presenteren we een prijsaanpassingsproces dat eindigt in een evenwichtsprijs terwijl het gestart mag worden in een willekeurige prijs. Dit resultaat kan voor willekeurige ruileconomieën behaald worden. Ten tweede dragen we een variabele dimensie herstart algoritme bij, dat uitgaande van een willekeurige prijs voor een willekeurige ruileconomie eindigt met een willekeurig precieze benadering van een evenwichtsprijs. De derde bijdrage in Hoofdstuk 2 betreft een alternatief bewijs van existentie van een. evenwichtsprijs met behulp van een zogenaamde intersectie-stelling. Deze intersectie-stelling is onlosmakelijk verbonden met de twee eerdere bijdragen. in Hoofdstuk 2.

Hoofdstuk 3, 'Evolution of populations and strategies', bevat twee modellen. Het eerste model beschrijft de evolutie van de samenstelling van een. populatie met verscheidene subgroepen. De centrale aanname daarbij is dat een proces van Darwiniaanse ('natumrlijke') selectie plaatsvindt. Dit houdt in dat veranderingen in de samenstelling van de populatie gedreven worden. door verschillen in fitnessniveaus tussen de subgroepen onderling. Een additionele aanname is dat het gedrag van de subgroepen genetisch voorbepaald is. Cruciaal is tenslotte dat fitnessniveaus niet vast hoeven te zijn, maar kunnen veranderen wanneer de samenstelling van de populatie verandert. Het tweede model beschrijft de evolutie van een populatie met verscheidene subgroepen die hun gedrag (strategie) kunnen aanpassen aan hun omgeving. Dit. 'aanpassen aan hun omgeving' interpreteren we als 'leren'. Hier verandert derhalve niet alleen de samenstelling van de populatie, maar ook de strategie van elke subgroep.

Voor beide modellen gebruiken we dezelfde drie oplossingsconcepten, het 'saturated' evenwicht, het evolutionair stabiele evenwicht en de gegeneraliseerde evolutionair stabiele toestand. We laten zien dat deze drie evolutionaire evenwichten vaste punten zijn voor de klassen van evolutionaire dynamieken die we in dit hoofdstuk behandelen. Verder behandelen we verbanden tussen. deze evolutionaire evenwichtsconcepten. met standaard evenwichtsconcepten zoals die bekend zijn uit de analyse van dynamische systemen, alsmede met evenwichtsconcepten zoals die bekend zijn uit de speltheorie.

Hoofdstuk 4, 'Changing payoffs or action sets', bevat twee speltheoretische modellen waar gedurende het verloop van een spel, hetzij de uitbetalingen, hetzij de actieverzamelingen van de spelers veranderen als gevolg van het spelverloop. In het eerste model in dit hoofdstuk bekijken we twee-persoonsspelen met zogenaamde 'vanishing actions'. In een dergelijk spel kunnen de actieverzamelingen. van beide spelers kleiner worden, aangezien elke speler 
elke actie die hij een zeker aantal perioden niet gebruikt heeft, verliest. Dit verliezen van een actie als gevolg van het niet gebruiken van deze actie hebben wij 'unlearning-by-not-doing' genoemd. We bekijken eerst nulsomspelen, en vervolgens bekijken we spelen met willekeurige uitbetalingen. We tonen de existentie van oplossingen aan voor enkele deelklassen van deze spelen, hetgeen wil zeggen dat we Nash evenwichten, alsmede optimale strategiën voor het zogenaamde 'limiting average reward' criterium, geven.

In het tweede model van Hoofdstuk 4 onderzoeken we aspecten van veranderende uitbetalingen aan de hand van zogenaamde differentiële spelen. Twee spelers krijgen stromen van uitbetalingen waarvan een vaste hoeveelheid opnieuw geïnvesteerd dient te worden verdeeld over twee activiteiten voor elke speler. Elke speler heeft een activiteit waarvan de uitbetaling uitsluitend afhangt van zijn eigen investering in die activiteit en een andere activiteit waarvan de uitbetaling niet alleen van zijn eigen investering afhangt, maar ook van de investering door de andere speler. Onder de aanname dat beide spelers hun (oneindige stroom) uitbetalingen willen maximaliseren, leiden we Nash evenwichten en zogenaamde open-loop strategiën af.

Hoofdstuk 5, 'Egalitarianism, potentials, and values', betreft coöperatieve speltheorie. Winsten of kosten gemaakt door een groep van samenwer-kende spelers, dienen verdeeld te worden over deze spelers. Een 'value' kan gezien worden als een verdeel-regel van dergelijke winsten of kosten van samenwerkende spelers voor arbitraire spelen. Een bekend voorbeeld van een value is de Shapley value die vele toepassing heeft in de economie en andere sociale wetenschappen. Een gemakkelijk te bevatten voorbeeld van een value is de egalitaire value waar bij alle winsten of kosten gelijkelijk verdeeld worden over alle spelers.

Onze bijdrage is gemotiveerd door een wens om een value te ontwerpen die een zekere vorm van egalitarisme bevat. We nemen daartoe aan dat nulspelers, dit zijn spelers die niets toevoegen aan het spel, toch meedelen in de winsten of kosten gemaakt door de groep van samenwerkende spelers, een vaste fractie $\alpha$ krijgen van de per-capita winst of kosten. Dit kan bewerkstelligd worden door middel van vrijwillige bijdragen aan een fonds dat ge-lijkelijk herverdeeld wordt, of door een proportionele belasting die herverdeeld wordt onder alle spelers. De parameter $\alpha$ kan gezien worden als een maatstaf voor het egalitarisme of voor de solidariteit. We bekijken values die deze eigenschap, genaamd $\alpha$-egalitarisme, alsmede enkele andere 'redelijke' eigenschappen, bezitten. Dit leidt voor elke $\alpha$ tot een value die wij de $\alpha$-egalitaire Shapley value genoemd hebben. De egalitaire value is een 
speciaal geval van een $\alpha$-egalitaire Shapley value waar $\alpha=1$, de Shapley value is dan weer een ander speciaal geval waar $\alpha=0$ te nemen. We leiden vervolgens een aantal zogenaamde 'axiomatische karakteriseringen' van de $\alpha$-egalitaire Shapley values af. Dit houdt in dat we een dergelijke value eenduidig vastleggen aan de hand van een (zo klein mogelijk) aantal 'redelijke' eigenschappen.

We gaan een stapje verder met een gedachte van 'belasting-innen-enherverdelen' om een meer algemene klasse van values af te leiden, die afhangen van een tupel van parameters. Teneinde dit te bereiken formuleren we zogenaamde 'potentialen'. Een potentiaal is een functie die aan elk coöperatief spel een uniek reëel getal toevoegt. Gegeven een tupel van parameters, definiëren we een potentiaal en verbinden er vervolgens een unieke value aan op de volgende manier. Elke speler ontvangt, wanneer de value wordt toegepast op een zeker spel, precies zijn marginale bijdrage aan de potentiaal van dat spel. We onderzoeken voorts verbanden tussen restricties op het tupel van parameters en eigenschappen welke de value heeft die juist door dit tupel vastgelegd wordt.

\subsection{Curriculum vitae}

Ik ben op 29 december 1961 in Heerlen geboren. Het grootste gedeelte van mijn scholing tot aan de universiteit vond plaats in Hoensbroek: kleuterschool bij juffrouw Monique en juffrouw Gerda, de Christus Koning en St. Cecilia lagere scholen, en tenslotte het Gymnasium $\beta$ aan het St. Janscollege. In 1980 mocht ik mij student econometrie noemen aan de Katholieke Hogeschool Tilburg, die gedurende mijn. negenjarig verblijf omgedoopt werd tot Katholieke Universiteit Brabant. Ik ben afgestudeerd in de specialisatie Wiskundige Economie. In 1990 begon ik een A.I.O.-project aan de Rijksuniversiteit Limburg. Wederom vond een namsverandering plaats zodat ik mu mijn graad van doctor mag ontvangen van de Universiteit Maastricht. Sedert 1989 ben ik werkzaam aan de Katholieke Universiteit Brabant als docent LV\&S. Op 1 oktober 1996 ben ik begonnen als post-doctoraat research fellow voor METEOR aan de Universiteit Maastricht. 


\subsection{Author index}

Acharya, S.N., 94, 135

Andreoni, J., $\quad 55,88$

Arrow, K.J., $\quad 11,26,48,63,64,85,93,94,135$

Arthur, W.B., 56, 85

Aumann, R.J., 109, 135

Basu, K., $\quad 81,85$

Binmore, K., $\quad 80,85$

Björnerstedt, J., $\quad 55,86$

Blackwell, D., $\quad 96,135$

Blanchard, O.J., 94, 135

Block, H.D., $\quad 11,26,48,63,64,85$

Bomze, E., $\quad 63,86$

Börgers, T., $\quad 55,86$

Boylan, R.T., $\quad 79,86$

Cabrales, A., $\quad 65,86$

Cheng, L., $\quad 126,136$

Chun, Y., $\quad 140,143,166$

Crawford, V., 54, 86

Debreu, G., $\quad 12,17,26,48,56,63,64,86$

Dekel, E., $\quad 65,86$

Derks, J., 154,166

Dierker, E., $\quad 64,86$

Doup, 'T.M., $13,14,48,49,57,65,76,86$

Dow, G.K., $\quad 56,86$

Dutta, B., $\quad 140,166$

Elzen, A.H. van den, $13,49,57,65,87$

Ferguson, T.S., $\quad 96,135$

Filar, J.A., $\quad 95,107,136$

Foster, D., $\quad 80,87$

Freidenfelds, J., 15, 44, 46, 47, 49

Friedman, D., $\quad 54,56,59,62,87$

Fudenberg, D., 80,87 
Gaunersdorfer, A., 81,87

Gilboa, I.,

$55,56,59,62,69,79,81,87$

Gillette, D.,

95,136

Hahn, F., $\quad 63,85$

Harker, P.T., $\quad 65,87$

Harris, C., $\quad 80,87$

Hart, S., $\quad 139-141,145-147,151,152,155,156,158,166$

Herings, P.J.J., 13, 15, 24, 28, 30, 35, 49

Hirsch, M.W., $\quad 59,60,68,71,84,87$

Ho, Y.C., 126,137

Hofbauer, J., $\quad 54-56,60,64,65,67,71,77,81,87,88$

Hurwicz, L., $11,26,48,64,85$

Jansen, W., $\quad 57,65,88$

Jonker, L.B., $\quad 53,76,90$

Joosten, R.A.M.G., 16, 49, 57, 67, 71, 88, 95, 136

Kalai, E., $\quad 145,146,177$

Kamiya, K., $\quad 13,49$

Kandori, M., $\quad 80,88$

Keenan, D., $\quad 27,49$

Knaster, B., $\quad 15,44,46,50$

Kuhn, H.W., $12-14,50$

Kuratowski, C., 15, 44, 46, 50

Laan, G. van der, $13-15,48,50,57,65,76,86$

Levhari, D., $\quad 126,136$

Mailath, G.J., $\quad 80,88$

Mantel, R., $\quad 12,17,26,50,56,63,88$

Mas-Colell, A., 139-141, 145-14.7, 151, 152, 155, 156, 158, 166

Matsui, A., $\quad 55,56,59,62,63,69,79,81,87,88$

Mazurkiewicz, S., $15,44,46,50$

Mertens, J.F., $\quad 95,108,136$

Metcalfe, J.S., $\quad 56,88$

Miller, J.H., $\quad 55,88$

Mirman, L., $\quad$ 126, 136

Möller, J., $\quad 94,136$

Monderer, D., $\quad 56,88,89$ 
Nachbar, J.H., $\quad 56,59,65,89$

Nash, J., $\quad 54,89$

Neyman, A., $\quad 95,108,136$

Nowak, A.S., 140,166

Oechssler, J., $\quad 55,56,89$

Pang, J.S., $\quad 65,87$

Perko, L., $\quad 59,89$

Peters, H., 95, 136, 154, 166

Price, G.A., $54,60,62,63,76,88$

Radzik, T., $\quad 140,166$

Raghavan, T.E.S., 95, 107, 136

Ray, D., $\quad 140,166$

Reinganum, J., $\quad 126,137$

Ritzberger, K., $\quad 59,62,81,89$

Rob, R., $\quad 80,88$

Robinson, J., $\quad 56,89$

Rosenmüller, J., $\quad 55,59,89$

Roth, A.E., 144,166

Saari, D.G., $\quad 27,50,57,65,89$

Samet, D., $\quad 145,146,177$

Samuelson, L., $\quad 53,55,59,79,80,85,89$

Samuelson, P., 11, 25-27, 51

Sarin, R., $\quad 55,86$

Scarf, H., $\quad 11-14,26-28,51$

Schelling, T., 56,89

Schlag, K., $\quad 55,56,90$

Schmeidler, D., 147,166

Scotchmer, S., $\quad 65,86$

Sela, A., $\quad 56,88$

Shapley, L.S., $\quad 56,88,89,95,137,139-142,144-148,150-158,166$

Sigmund, K., $\quad 54,56,60,71,88$

Silverberg, J., $\quad 56,90$

Simon, C., $\quad 27,50$

Smale, S., $\quad 12,26-28,51,59,60,68,71,84,87$ 
Sobel, J., $\quad 65,86$

Sonnenschein, H., 12, 17, 26, 51, 56, 63, 90

Spence, A.M., $\quad 126,137$

Starr, A.W., $\quad 126,137$

Summers, L.H., $\quad 94,136$

Swinkels, J., $\quad 55,59,90$

Talman, A.J.J., $13-16,48-51,57,76,86,87$

Taylor, P.D., $\quad 53,76,90$

Thuijsman, F., $95,109,136,137$

Tijs, S.H., $\quad 147,167$

Uzawa, H., $12,26,51,63,64,90$

Van Damme, E.C.C., $\quad 63,76,86,90$

Van der Heyden, L., 13, 50

Vega-Redondo, F., $\quad 126,133,137$

Vrieze, O.J., $106,107,137$

Walras, L., $\quad 11,25,27,51,64$

Weibull, J.W., $\quad 55,59,62,81,85,86,89,90$

Weissing, F.J., 61, 65, 90

Yang, Z., 15, 24, 51

Young, H.P., 80, 87, 90, 139, 140, 142-145, 150, 167

Zeeman, E.C., $55,65,90,91$

Zhang, J., $\quad 53,55,59,89$ 

